\title{
Regulation of Citric Acid Cycle Enzymes and Related Pathways in the Skeletal Muscle of Hibernating Richardson's Ground Squirrels, Urocitellus Richardsonii
}

\author{
By \\ Stuart Russell Green \\ B.Sc. (Hons.) Carleton University, 2015
}

M.Sc. Carleton University, 2017

A Thesis Submitted to the Faculty of Graduate Studies and Research in partial fulfillment of the requirements for the degree of

Doctor of Philosophy

Department of Biology

Carleton University

Ottawa, Ontario, Canada

(C) Copyright 2021, Stuart Green 


\begin{abstract}
Richardson's ground squirrels (Urocitellus richardsonii) are small rodents inhabiting western Canada that spend a large portion of their life in hibernation. Hibernation is accompanied by a profound drop in body temperature to a minimum of $2-3{ }^{\circ} \mathrm{C}$ and a notable shift from carbohydrate to lipid consumption that involves large-scale rearrangements of central metabolic processes. This thesis investigated the regulation of key enzymatic checkpoints in the citric acid cycle (CAC) as well as enzymes that shuttle substrates into the CAC in skeletal muscle of ground squirrels during hibernation. Initial work investigated regulation of the pyruvate dehydrogenase complex (PDC) that bridges glycolysis and the CAC. Muscle PDC showed few changes in properties in terms of activity and inhibitory phosphorylation of the enzyme. This was in stark contrast to liver where strong suppression of PDC activity occurred during hibernation correlated with increased inhibitory phosphorylation on serine-300. This then led to investigation of two crucial irreversible regulatory steps of the CAC in the muscle: citrate synthase (CS) and the $\alpha$ ketoglutarate dehydrogenase complex (KGDC). CS activity decreased significantly during hibernation. This correlated with decreased lysine succinylation of CS that reflected increased SIRT5 levels, the enzyme responsible for desuccinylase activity in mitochondria. KGDC also showed decreased affinity for coenzyme A in hibernating squirrels and marked differences in posttranslational modifications including increased tyrosine phosphorylation on all three enzyme subunits and increased serine phosphorylation on E2 subunit. Stimulating the action of endogenous protein kinases demonstrated decreased affinity for coenzyme A. Finally, regulation of muscle glutamate dehydrogenase (GDH) was analyzed to ascertain how GDH regulation mediated the flow of $\alpha$-ketoglutarate into the CAC from amino acid catabolism. Most GDH kinetic parameters were unaffected between hibernating and euthermic states, except that
\end{abstract}


glutamate affinity was substantially lower at $8^{\circ} \mathrm{C}$ (a physiologically relevant temperature) for the enzyme from hibernating squirrels. GDH from hibernating animals also exhibited significantly higher ADP-ribosylation, suggesting a regulatory mechanism for modulating GDH. Taken together these findings suggest that enzymatic regulation in Richardson's ground squirrel muscle is actively mediated by a variety of posttranslational mechanisms of the CAC and related enzymes to coordinate metabolic suppression during hibernation. 


\section{Preface}

The following integrated thesis describes data from four different research projects that have been written as complete papers. Two of these projects have already been published in peerreviewed journals and their inclusion in this thesis is granted in accordance with the copyright agreement set forth by the publisher (see Elsevier copyright policy for more information). A third chapter has been submitted and is under review at the time of submission of this thesis. The bibliographic information for the two published articles is as follows:

\section{Chapter 2}

Green, S.R. and Storey, K.B. (2021) Functional and post-translational characterization of pyruvate dehydrogenase demonstrates repression of activity in the liver but not skeletal muscle of the Richardson's ground squirrel (Urocitellus richardsonii) during hibernation. Journal of Thermal Biology. 99. DOI: 10.1016/j.jtherbio.2021.102996

\section{Chapter 4}

Green, S.R. and Storey, K.B. (2020) Regulation of the $\alpha$-ketoglutarate dehydrogenase complex during hibernation in a small mammal, the Richardson's ground squirrel (Urocitellus richardsonii). Biochimica et Biophysica Acta (BBA) - Proteins and Proteomics. 1868 (9). DOI: 10.1016/j.bbapap.2020.140448

\section{Chapter 3}

The following data chapter has been submitted and is currently under consideration at the time of writing as detailed below:

Cryobiology

Green, S.R. and Storey, K.B. (2021) Citrate synthase in hibernating Richardson's ground squirrels (Urocitellus richardsonii) displays altered functional properties. Submitted April 7 2021. Manuscript No. CRYO-D-21-00094 


\section{Acknowledgements}

Wow, hard to believe it's finally here. When I first showed up in the Storey lab as a fresh-faced undergrad just out of my first year over 9 years ago I had no idea that I would spend the better part of a decade working in and out of this lab. Over the years, I have seen so many people come and go that it seems a little bit surreal to think that soon I will be another name on a blue book on the shelves of Steacie building room 511 like so many who have come before me. About a year ago, I tried to count in my head all the people from my undergrad up until then that I had met through my experiences in the lab and I got up to around 60 before I lost count. With that being said, this may be the most difficult part of my thesis to write in part because I don't know how I could ever possibly thank everyone who has helped guide me and make my time here so enjoyable in a scant page or two (but I will do my best).

First, I must thank my supervisor Ken Storey for his astounding commitment to his students (myself included) and helping to give invaluable advice and guidance in designing the projects that make up this thesis and teaching me to be a better scientist in general. I would also be remiss if I didn't also give thanks to Jan Storey for helping to improve my writing through her thorough and incisive editing and commentaries of my manuscripts and thesis chapters, even if in the process they were returned to me in mostly red text. I must also thank Dr. Tim Xing and Dr. Charles Darveau for taking time out of their busy schedules to serve on my thesis advisory committee for both my Master's and Doctoral degrees over the past six years and in doing so have helped to significantly improve the contents of this thesis. Special thanks to Neal Dawson and Ryan Bell for taking the time to teach me the basics of enzymology and how to hold a pipette so many years ago, none of this would have been possible without you. 
Thanks to the many friends that I have made along the way. Thanks Sam for never failing to update me on the latest 'True-Facts' video and helping me avoid the dreaded OC-Transpo by offering me rides whenever possible. Hanane, thanks for giving me someone to commiserate about the dreaded 'reviewer 2' and putting up with constant crunching of sunflower seeds. Thanks to Bryan for teaching me the ropes on PCR on for the endless LOTR and Forrest Gump quotes. Thanks Mike S. for letting me polish up your work and your lab memes that continue to adorn the office. Thanks Christie for your help with the job search and your wisdom in purifications. Thanks Trong for spotting me at the gym the one or two times that I went and teaching me that it is indeed possible to run $100 \mathrm{~km}$ in under 3 hours. Thanks Zephanie for offering all of the snacks (even when they happened to taste suspiciously like seaweed). Thanks Jess for all your hard work in putting together the lab softball team. Thanks Janelle for the Bob Ross bobble-head, enough said. Thanks Rasha for teaching me how to work the Luminex machine and your 'inspired' vocal renditions of pop songs. Thanks Aline, Anchal, Aakriti and Sarah for the great lunch conversations and giving me people to talk to while waiting for the microwave to heat my food in Ken's office. Thanks Tighe and Gurjit for the conversations/debates about anything and everything. Thanks to everyone else I have met over the years who I do not have space to mention here.

Finally, I want to thank my parents and my brothers for their love and support (and patience) throughout my undergrad and grad studies and reminding me to have a life outside of school. 


\section{Table of Contents}

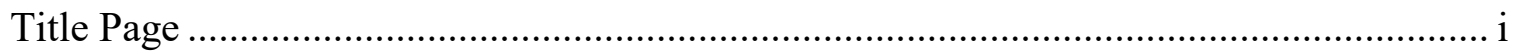

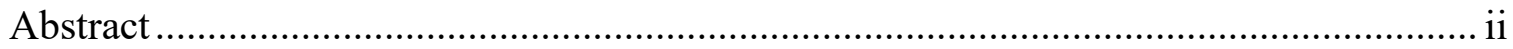

Preface

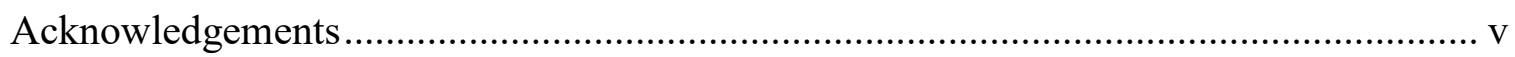

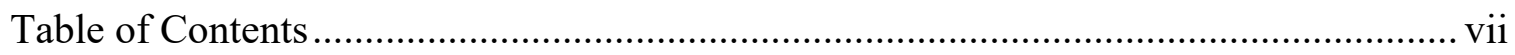

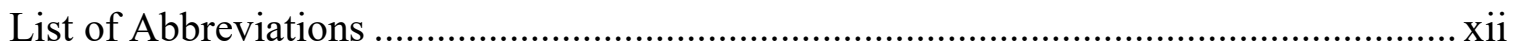

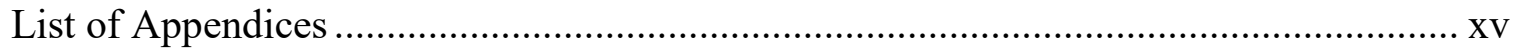

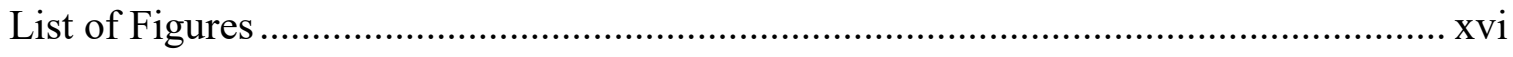

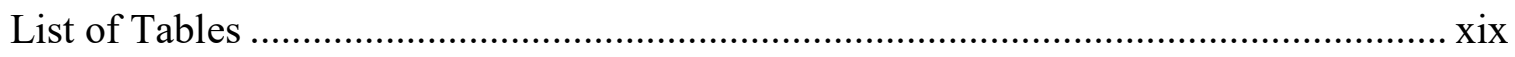

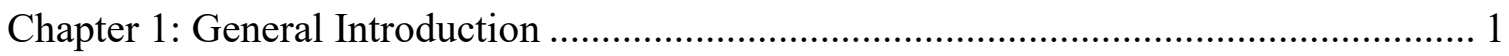

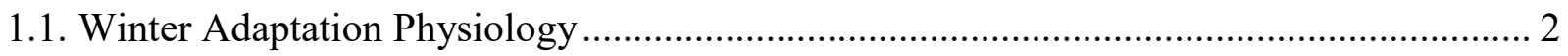

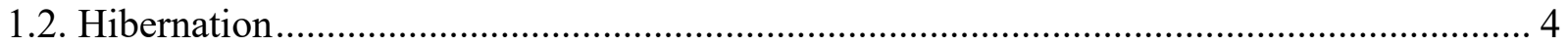

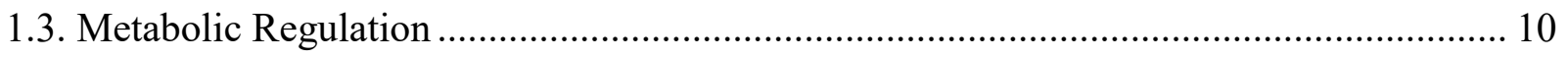

1.4. Enzymatic Regulation in the Richardson's Ground Squirrel during Hibernation ............. 14

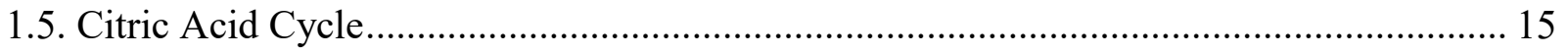

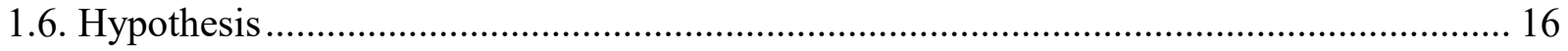

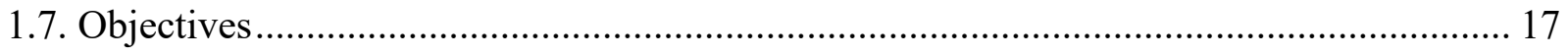

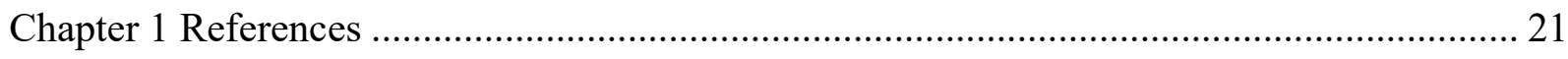




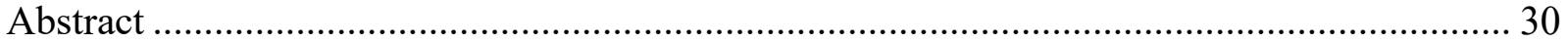

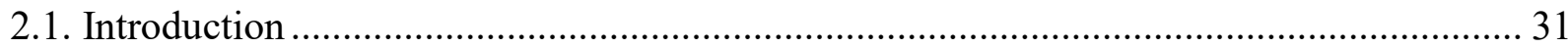

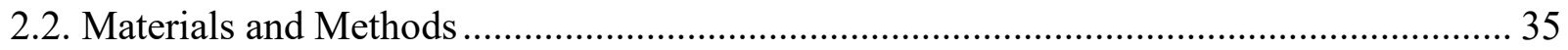

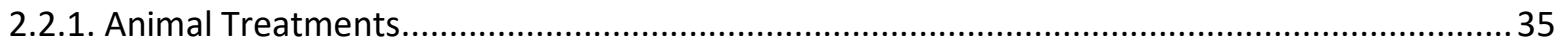

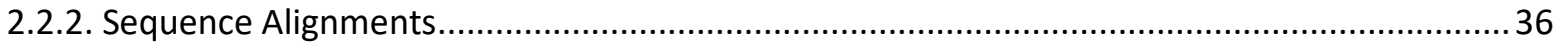

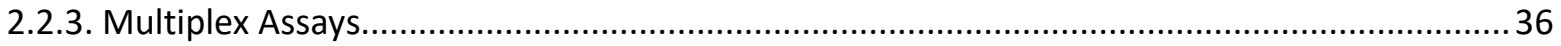

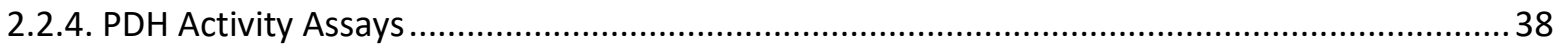

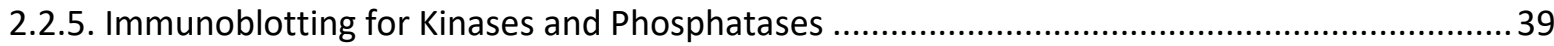

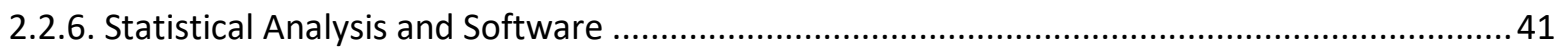

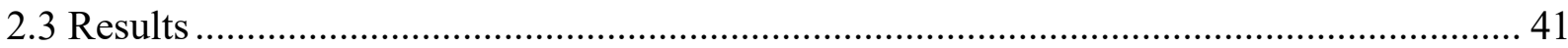

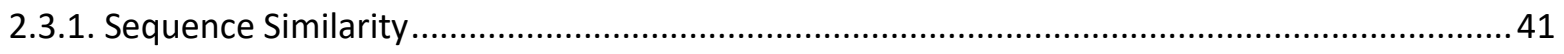

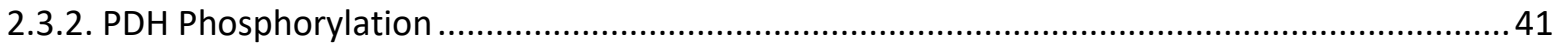

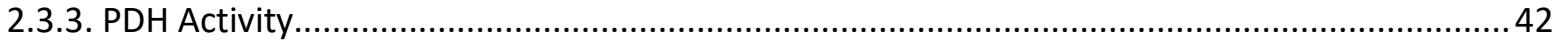

2.3.4. PDH Kinase and Phosphatase Expression ....................................................................... 42

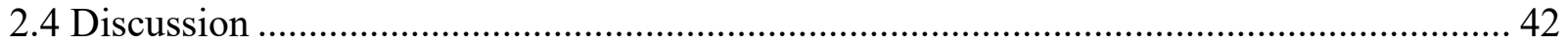

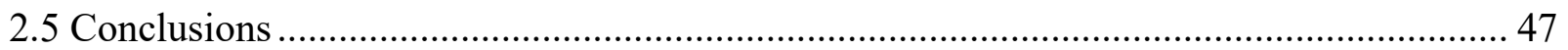

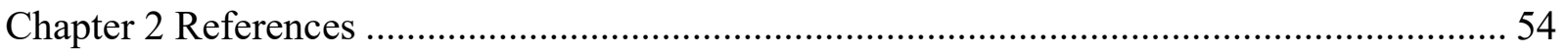

Chapter 3: Citrate Synthase Regulation in Muscle during Hibernation ......................... 58

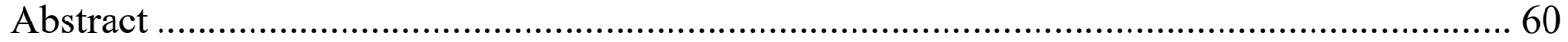

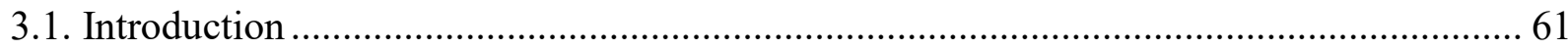

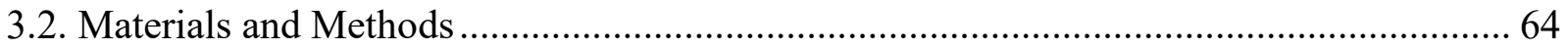

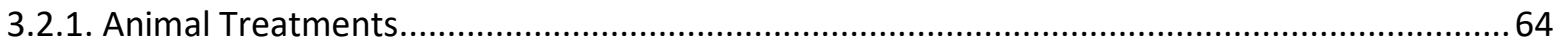

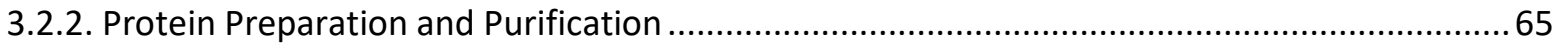

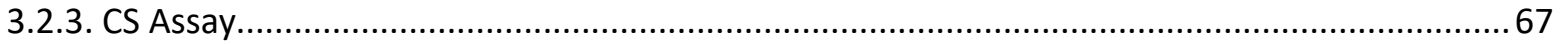




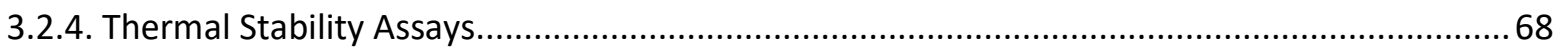

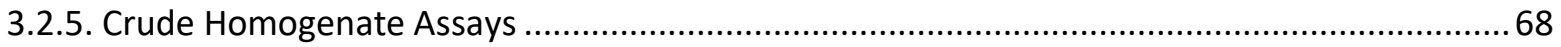

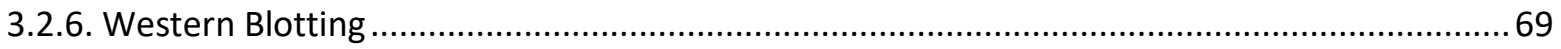

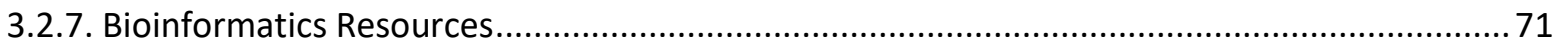

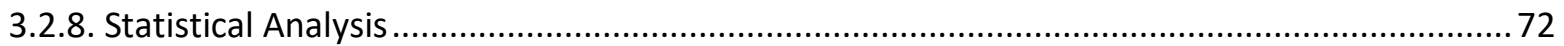

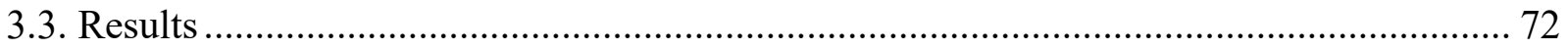

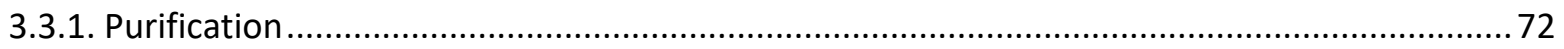

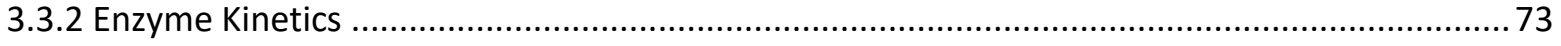

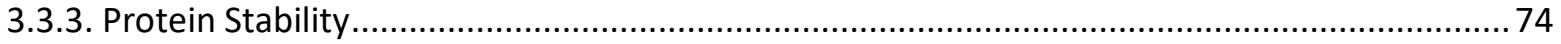

3.3.4. Western Blots for Post-translational Modifications ........................................................... 74

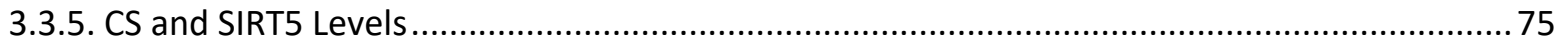

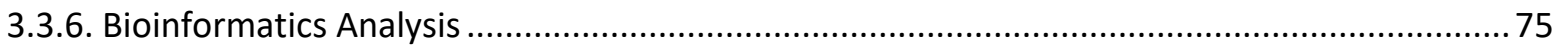

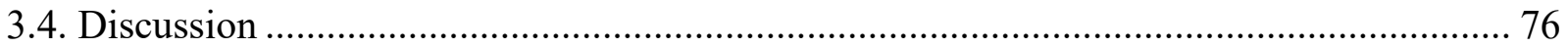

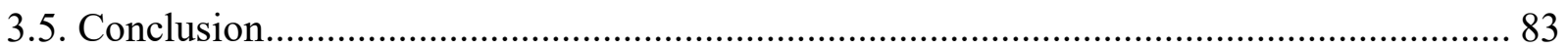

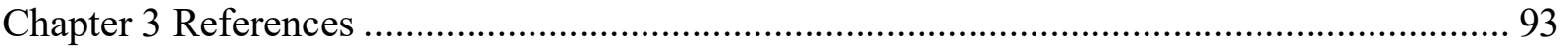

Chapter 4: Regulation of Muscle KGDC in Hibernation............................................. 98

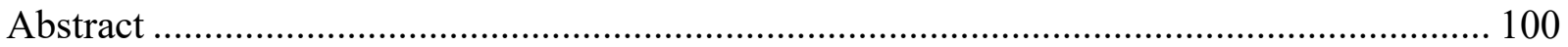

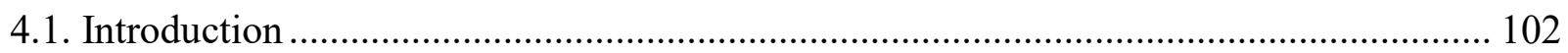

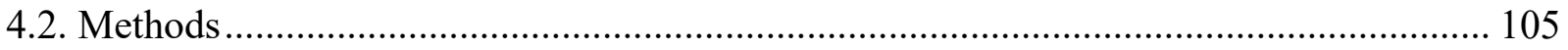

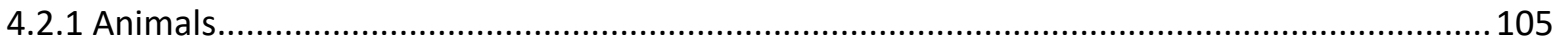

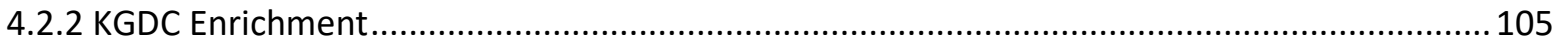

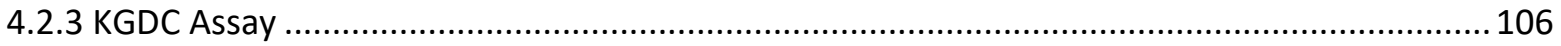

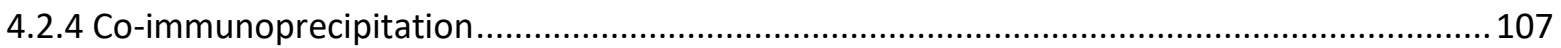

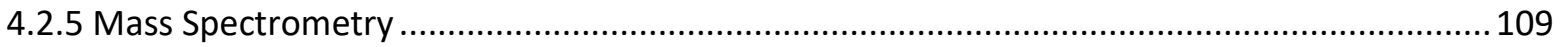

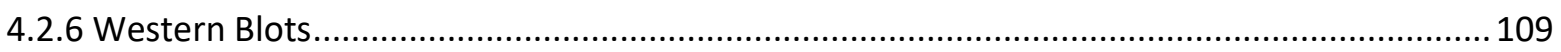

4.2.7 Incubations to Stimulate Endogenous Kinases and Phosphatases ......................................111

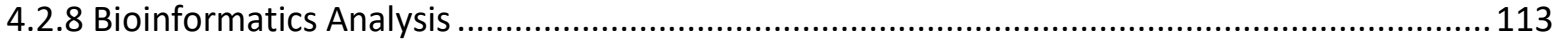




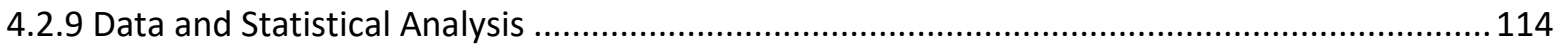

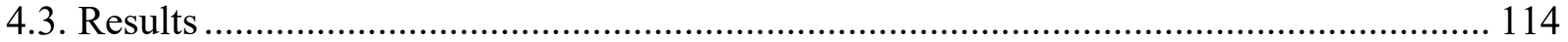

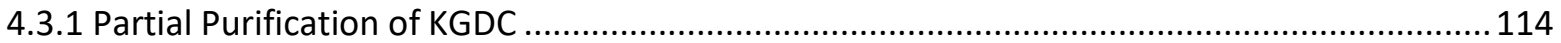

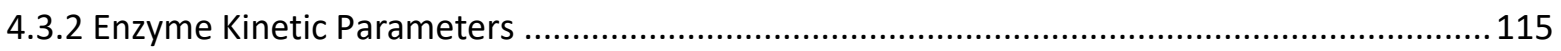

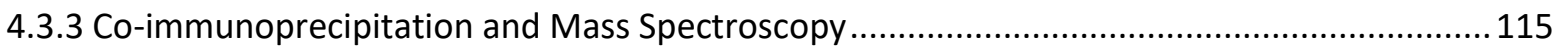

4.3.4 Evaluation of Post-translational Modifications.................................................................116

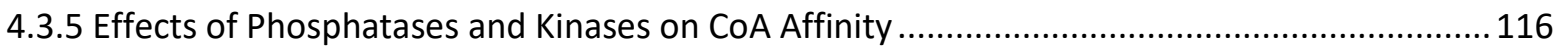

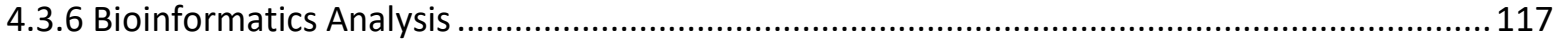

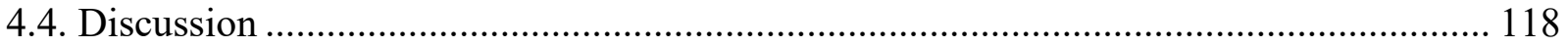

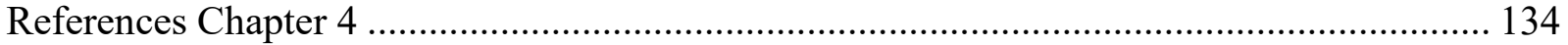

Chapter 5: Regulation of Muscle GDH during Hibernation ........................................ 139

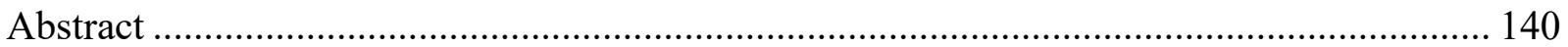

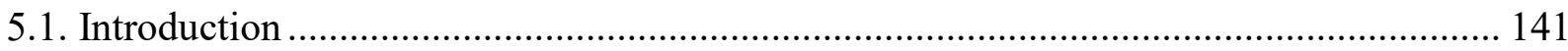

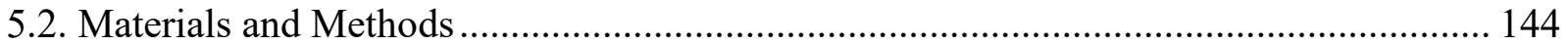

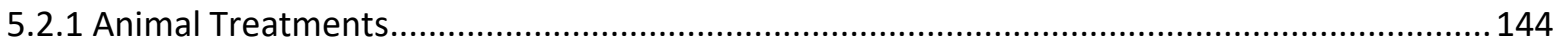

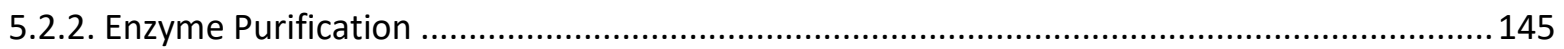

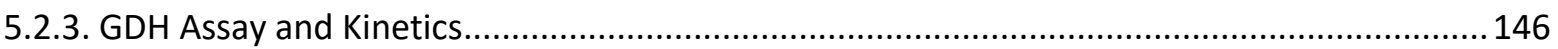

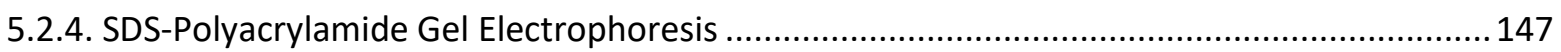

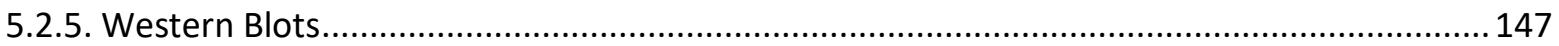

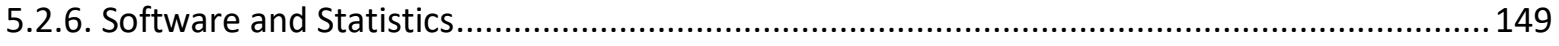

5.2.7. In Silico ADP-Ribosylation Prediction ................................................................................. 149

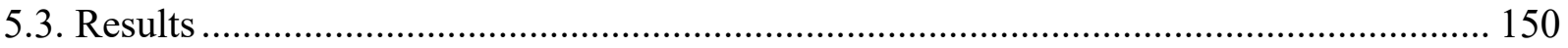

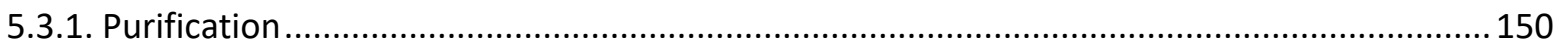

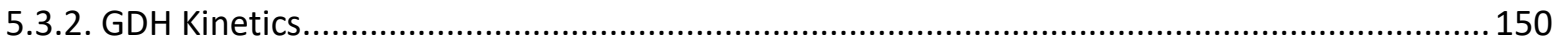

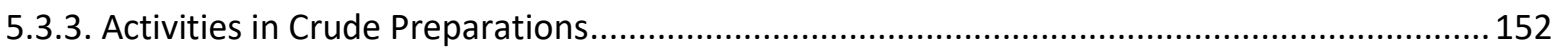

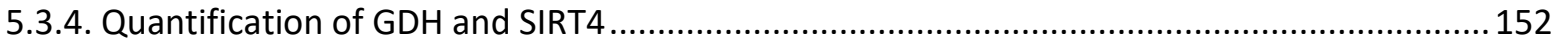

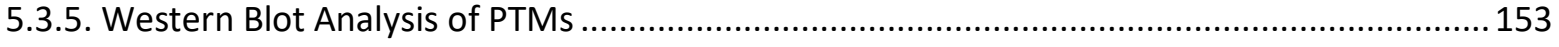


5.4 Discussion

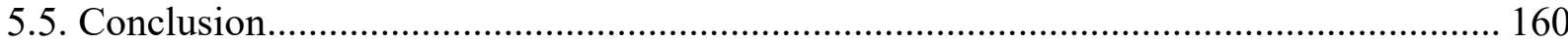

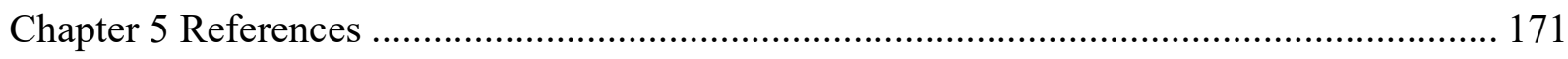

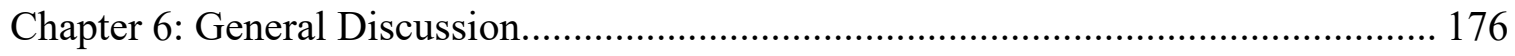

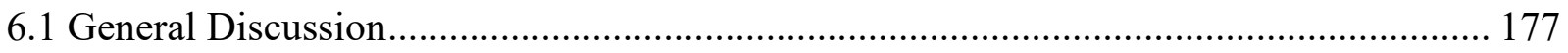

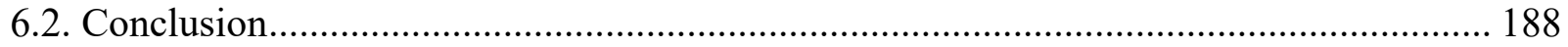

6.3. Future Directions ............................................................................................ 189

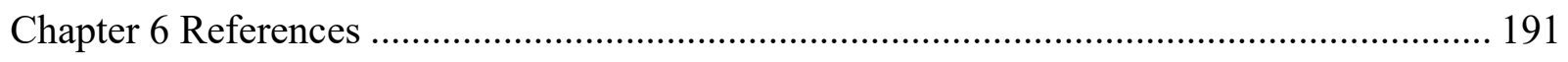

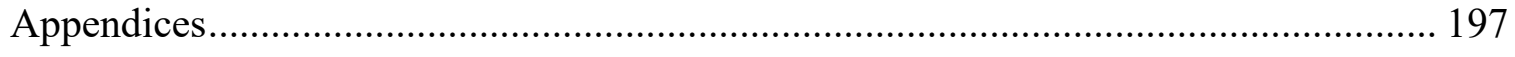




\section{List of Abbreviations}

$\begin{array}{ll}\text { 13-LGS } & \text { 13-Lined Ground Squirrel } \\ \text { ADP } & \text { Adenosine Diphosphate } \\ \text { ADPR } & \text { ADP-ribosylation } \\ \text { ANOVA } & \text { Analysis of Variance } \\ \text { ART } & \text { ADP-ribosyl Transferase } \\ \text { ATP } & \text { Adenosine Triphosphate } \\ \text { BCKDC } & \text { Branched Chain Keto-acid Dehydrogenase Complex } \\ \text { CAC } & \text { Citric Acid Cycle } \\ \text { CaCl2 } & \text { Calcium Chloride } \\ \text { cAMP } & \text { 3',5'-cyclic Adenosine Monophosphate } \\ \text { cGMP } & \text { 3',5'-cyclic Guanosine Monophosphate } \\ \text { CM } & \text { Carboxymethyl cation exchange chromatography material } \\ \text { CoA } & \text { Coenzyme A } \\ \text { Co-IP } & \text { Co-immuno Precipitation } \\ \text { CS } & \text { Citrate Synthase } \\ \text { CS-KMT } & \text { Citrate Synthase Lysine Methyl Transferase } \\ \text { DLD } & \text { Dihydrolipoamide Dehydrogenase (E3 subunit of KGDC/PDC) } \\ \text { DLST } & \text { Dihydrolipoyl Succinyltransferase (E2 subunit of KGDC) } \\ \text { DMP } & \text { Dimethyl Pimelimidate } \\ \text { DTNB } & 5,5 \text {-dithio-bis-(2-nitrobenzoic acid) (Ellman's Reagent) } \\ \text { ECL } & \text { Enhanced Chemiluminescence } \\ \text { EDTA } & \text { Ethylene Diamine Tetraacetic Acid } \\ \text { EGTA } & \text { Ethylene Glycol Tetraacetic Acid } \\ \text { ELISA } & \text { Enzyme-linked Immunosorbent Assay } \\ \text { CA } & \end{array}$

FAD/FADH 2 Flavin Adenine Dinucleotide (oxidized/reduced) 


\begin{tabular}{|c|c|}
\hline Fe & Fragment crystallizable region of immunoglobulin \\
\hline GDH & Glutamate Dehydrogenase \\
\hline Glu & Glutamate \\
\hline GTP & Guanosine Triphosphate \\
\hline$I_{50}$ & Inhibitor concentration causing half maximal inhibition \\
\hline IgG & Immunoglobulin class $\mathrm{G}$ \\
\hline I-TASSER & Iterative Threading ASSEmbly Refinement \\
\hline $\boldsymbol{K}_{\boldsymbol{A}}$ & Activator concentration resulting in half maximal activation \\
\hline KCl & Potassium Chloride \\
\hline kDa & Kilodalton \\
\hline KG & $\alpha$-ketoglutarate \\
\hline KGDC & $\alpha$-Ketoglutarate Dehydrogenase Complex \\
\hline $\boldsymbol{K}_{m}$ & $\begin{array}{l}\text { Michaelis Constant (substrate concentration resulting in half maximal } \\
\text { activity }\end{array}$ \\
\hline LC/MS/MS & Liquid Chromatography - Tandem Mass Spectrometry \\
\hline Lys & Lysine \\
\hline MARylation & Mono-ADP-Ribosylation \\
\hline MCU & Mitochondrial Calcium Uniporter \\
\hline MDa & Megadalton \\
\hline MES & 2-(N-morpholino)ethanesulfonic acid \\
\hline $\mathrm{MgCl}_{2}$ & Magnesium Chloride \\
\hline MS & Mass Spectrometry \\
\hline OAA & Oxaloacetate \\
\hline OGDH & Oxoglutarate Dehydrogenase (E1 subunit of KGDC) \\
\hline $\mathbf{N A D}^{+} / \mathbf{N A D H}$ & Nicotinamide Adenine Dinucleotide oxidized/reduced \\
\hline $\mathbf{N A D P}^{+} / \mathbf{N A D P H}$ & NAD Phosphate oxidized/reduced \\
\hline $\mathrm{Na}_{3} \mathrm{VO}_{4}$ & Sodium Orthovanadate \\
\hline PARylation & Poly-ADP-Ribosylation \\
\hline
\end{tabular}




\begin{tabular}{ll} 
PBS & Phosphate Buffered Saline \\
PDC & Pyruvate Dehydrogenase Complex \\
PDH & Pyruvate Dehydrogenase (E1 subunit) \\
PDK & Pyruvate Kinase \\
PDP1 & Pyruvate Dehydrogenase Phosphatase Catalytic Subunit \\
PEG & Polyethylene Glycol \\
PEP & Phosphoenolpyruvate \\
pI & Isoelectric point \\
Pi & Inorganic Phosphate \\
PMSF & Phenylmethylsulfonylfluoride \\
PTM & Posttranslational Modification \\
PVDF & Polyvinylidene Fluoride \\
Q10 & Temperature co-efficient \\
SDS-PAGE & Sodium Dodecyl Sulfate Polyacrylamide Gel Electrophoresis \\
SIRT & Sirtuin \\
TBS & Tris-buffered Saline \\
TBST & Tris-buffered Saline with Tween-20 \\
TPP & Thiamine Pyrophosphate \\
Tris & Tris(hydroxymethyl)aminomethane \\
$\boldsymbol{V}_{\boldsymbol{m a x}}$ & Maximal enzyme velocity \\
WAT & White Adipose Tissue \\
& \\
\hline &
\end{tabular}




\section{List of Appendices}

Appendix A: Chapter 3 Supplementary Data and Figures. 198

Appendix A.1. Standard curve of coenzyme A amounts plotted against absorbance at $412 \mathrm{~nm}$ with $0.1 \mathrm{mM}$ DTNB in a final volume of $200 \mu \mathrm{L} 50 \mathrm{mM}$ Tris buffer $\mathrm{pH} 8.0$ at $22{ }^{\circ} \mathrm{C}$

Appendix A.2. Sequences and alignment between 13-lined ground squirrel CS (Ictidomys tridecemlineatus) and human (Homo sapiens)..... 200

Appendix A.3. Full blot image demonstrating the molecular weight of citrate synthase from euthermic and hibernating $U$. richardsonii muscle tissue lysates. 202

Appendix B: Chapter 4 Supplementary Data and Figures 203

Supplementary Fig. B.1. Bioinformatics resources for OGDH subunit of KGDC 205

Supplementary Fig. B.2. Bioinformatics resources for DLST subunint of KGDC 206

Supplementary Fig. B.3. Bioinformatics resources for DLD subunit of KGDC 207

Appendix C: Chapter 5 Supplementary Data and Figures 208

Appendix C.1. Identification of GDH band in crude lysates compared to purified GDH .... 209 Appendix C.2. ADPredict ADP-ribosylation prediction tool full scoring output for all acidic residues in I. tridecemlineatus GDH sequence 210

Appendix D: Publication List 212

Published: 213

Submitted/Under Review: 214 


\section{List of Figures}

Fig. 1.1. Schematic diagram of the enzyme-mediated reactions of the citric acid cycle and related pathways. 20

Fig. 2.1. PDH E1 $\alpha$ subunit protein amino acid sequence alignment of a ground squirrel species (Ictidomys tridecemlineatus) compared to the human sequence demonstrating the three conserved regulatory phosphorylation sites. 49

Fig. 2.2. Graphical representation of the relative levels of phosphorylation at three conserved regulatory phosphorylation sites in the PDH E1 $\alpha$ subunit comparing the euthermic state to hibernation in Urocitellus richardsonii in the skeletal muscle and liver. 50

Fig. 2.3. Graphical representation of the relative levels of PDH activity in the euthermic and hibernating U. richardsonii muscle and liver extracts.

Fig. 2.4. Graphical representation of the relative levels of the four PDKs and PDP1 in the liver of euthermic and hibernating $U$. richardsonii.

Fig. 2.5. Graphical representation of the relative levels of the four PDKs and PDP1 in the muscle of euthermic and hibernating $U$. richardsonii...... 53

Fig. 3.1. Coomassie stained 10\% SDS-PAGE gels demonstrating typical CS purifications from euthermic and hibernating $U$. richardsonii muscle tissue

Fig. 3.2. Graphical representation of the specific activity of CS in crude euthermic and hibernating $U$. richardsonii skeletal muscle tissue lysates at 8,22 and $37^{\circ} \mathrm{C}$ 88

Fig. 3.3. Graphical representation of purified CS thermal stability at $60^{\circ} \mathrm{C}$ from lysates prepared from euthermic and hibernating U. richardsonii skeletal muscle. 89 
Fig. 3.4. Graphical representation of the relative levels of various PTMs in euthermic and hibernating U. richardsonii CS determined through western blot analysis 90

Fig. 3.5. Graphical representation of the relative levels of CS and SIRT5 in the euthermic and hibernating $U$. richardsonii skeletal muscle lysates 91

Fig. 3.6. Bioinformatics analysis of potential lysine succinylation sites on CS of Ictidomys tridecemlineatus using the conserved domain database and protein modelling

Fig. 4.1. Western blot identification of the subunits of the KGDC enriched using chromatographic techniques from euthermic $U$. richardsonii skeletal muscle. 128

Fig. 4.2. SDS-PAGE gel demonstrating the purification and subunit identification of euthermic KGDC through co-immunoprecipitation from euthermic $U$. richardsonii skeletal muscle129

Fig. 4.3. Graphical representation of the relative levels of various PTMs on the OGDH (E1) subunit from euthermic and hibernating U. richardsonii KGDC determined through coimmunoprecipitation and western blotting 130

Fig. 4.4. Graphical representation of the relative levels of various PTMs on the DLST (E2) subunit from euthermic and hibernating U. richardsonii KGDC determined through coimmunoprecipitation and western blotting

Fig. 4.5. Graphical representation of the relative levels of various PTMs on the DLD (E3) subunit from euthermic and hibernating $U$. richardsonii KGDC determined through coimmunoprecipitation and western blotting 132

Fig. 4.6. Comparing the effects of incubations in conditions activating for various protein kinases and phopshatases on KGDC affinity for CoA in U. richardsonii muscle lysates 
Fig. 5.1. Coomassie stained 10\% SDS-PAGE gel demonstrating the purification of GDH from euthermic $U$. richardsonii muscle tissue 164

Fig. 5.2. Graphical comparison of $U$. richardsonii skeletal muscle GDH substrate affinity in the glutamate consuming direction of the reaction between the euthermic and hibernating purified enzyme at 8,22 and $37^{\circ} \mathrm{C}$. 165

Fig. 5.3. Graphical comparison of $U$. richardsonii skeletal muscle GDH substrate affinity in the $\alpha$-ketoglutarate consuming direction of the reaction between euthermic and hibernating purified enzyme at 8,22 and $37^{\circ} \mathrm{C}$ 166

Fig. 5.4. Graphical representation of the specific activity of $U$. richardsonii GDH in both directions of the reaction at $22{ }^{\circ} \mathrm{C}$ in crude tissue lysates. 167

Fig. 5.5. Graphical representation of the relative levels of GDH and SIRT4 in U. richardsonii skeletal muscle homogenates determined through western blotting comparing euthermic to hibernating animals 168

Fig 5.6. Graphical representation of the relative levels of various PTMs on GDH purified from euthermic and hibernating U. richardsonii determined through western blotting.... 169

Fig. 5.7. ADP-ribosylation prediction using the ADPredict online bioinformatics resource compared to a conserved protein domain map demonstrating the location of the putative ADPribosyl sites on the GDH sequence of I. tridecemlineatus. 170 


\section{List of Tables}

Table 3.1. Representative purification schemes of CS from control euthermic and hibernating $U$. richardsonii skeletal muscle $\mathbf{8 5}$

Table 3.2. Kinetic parameters of purified CS from euthermic and hibernating U. richardsonii at 8,22 and $37^{\circ} \mathrm{C} \quad \mathbf{8 6}$

Table 4.1. Representative partial purification scheme of the KGDC complex from skeletal muscle of $U$. richardsonii $\mathbf{1 2 6}$

Table 4.2. Kinetic parameters of enriched KGDC from euthermic and hibernating $U$. richardsonii at 8,22 and $37^{\circ} \mathrm{C} \quad \mathbf{1 2 7}$

Table 5.1. Representative purification of GDH from skeletal muscle of hibernating $U$. richardsonii $\mathbf{1 6 2}$

Table 5.2. Effects of activators and inhibitors at 8,22 and $37{ }^{\circ} \mathrm{C}$ on euthermic and hibernating U. richardsonii $\mathrm{GDH}$ in both directions of the reaction 163 


\section{Chapter 1: General Introduction}




\subsection{Winter Adaptation Physiology}

The biodiversity and population density of terrestrial life on Earth is not evenly distributed across the Earth's surface, but rather tends to be concentrated in areas close to the equator where heat and water are abundant (Bar-On, Phillips, \& Milo, 2018). The main difficulties inherent to colonizing habitats at extreme latitude lie in the scarcity of food and freezing temperatures during the winter season stemming from the limited hours of sunlight. Indeed, the difficulty imposed by lack of sunlight and cold conditions can make it nearly impossible for members of certain taxa to survive, especially for those incapable of controlling their body temperature. For example, despite its enormous surface area, Antarctica is home to only one free-living insect species, Belgica antarctica, a flightless dipteran (Usher \& Edwards, 1984). While the limitations of living in extreme environments might seem insurmountable at first, species that have the capacity to live in these harsh climates are rewarded by the relative rarity of inter-species competition in these habitats and once they become widely established it can be difficult for other species to displace them from the highly specialized ecological niche that they have come to dominate.

The threat imposed by freezing makes temperate climates challenging for animals to survive. This is particularly true among ectothermic animals that lack the capacity to alter their body temperature to maintain physiological processes at optimal conditions and prevent body fluids from freezing. While some of these animals have met this challenge successfully by developing freeze-tolerance or freeze-avoidance strategies, the diversity of ectothermic species decreases rapidly when traveling further north (Pincheira-Donoso, Bauer, Meiri, \& Uetz, 2013; Storey \& Storey, 2017). Only one species of lizard inhabits Ontario and even then it is only located at the Southernmost tip of the province (Quirt, Blouin-Demers, Howes, \& Lougheed, 
2006). Gradually reptile and amphibian species become rarer as one travels further north in North America until only one frog species is left; the wood frog (Rana sylvatica) that enjoys a very wide distribution all across the boreal forest of Canada and as far as north as Alaska thanks to the absence of competitors in its range and its robust freeze-tolerance capabilities (Costanzo, Reynolds, do Amaral, Rosendale, \& Lee, 2015; Costanzo, 2019). While mammalian and avian species also become less diverse travelling further north, the restrictions are not as noticeable with numerous species found north of the Arctic Circle. The capacity to maintain a constant body temperature, allows these animals to stay active for sufficiently long periods of time during the year to forge for the food necessary to survive and also helps to prevent their bodies from freezing during the winter (Bennett \& Ruben, 1979). The drawback to endothermy is the increase in fuel consumption needed. While maintenance of a constant set ideal body temperature helps maintain the animal in an active state where physiological processes are optimally suited, it also incurs large energetic penalties that are especially disadvantageous when food access is limited during the winter months (Brashears, Hoffman, \& DeNardo, 2017). This is particularly problematic for small animals that have a high surface area to volume ratio as they must maintain a high metabolic rate per unit of body mass to fight the increased heat loss (Fristoe et al., 2015). This has resulted in numerous animal species adopting extreme behavioral and physiological adaptations to survive these winter conditions.

The first and most straightforward method of coping with the reality of winter is to simply leave the area before winter comes and move to a warmer locale. Migration is a common strategy among more highly mobile animals as they can benefit from reduced competition during the summer months in the Arctic or more temperate climates while avoiding the lack of food that is attendant with winter in these locations. The most extreme example of this behavior is 
observed in a bird species, the arctic tern, that breeds above the Arctic circle before wintering in Antarctica, so that it can make take advantage of both the boreal and austral summer seasons (Fijn, Hiemstra, Phillips, \& Van Der Winden, 2013). The principal motivation for animals to make these journeys is the promise of finding better feeding grounds; however, this is also associated with a high energetic cost and typically involves the consumption of vast quantities of lipids for the animals to complete their migration. Indeed, one study measured a $52 \%$ decline in lipid levels in migrating geese over the migration season (Bromley \& Jarvis, 1993). Migration tends to be particularly common in birds as opposed to mammals despite the metabolic similarities between these two taxa, both having high metabolic rates and being endothermic. This is likely due to the fact that flying tends to be much more rapid and energetically efficient when adjusted for distance traveled and comparing animals of the same weight (Tucker, 1970). Indeed, migration in mammals is often found in bats, the only mammalian taxon capable of true flight, although hibernation is also employed (Fleming, 2019; Saleem, Al-attar, \& Storey, 2021). Because migration is preferred among avian species, few birds have developed a true hibernation response with only one known species (the common poorwill, Phalaenoptilus nuttallii) spending multiple weeks to months at a reduced body temperature (Jaeger, 1949; Woods, Czenze, \& Brigham, 2019). Torpor and hibernation tends to be much more common among mammals as a means to survive the challenges imposed by winter (Geiser \& Ruf, 1995; Ruf \& Geiser, 2015).

\subsection{Hibernation}

Hibernation in mammals is a common mechanism for coping with the reduced food availability of winter. Related to hibernation is daily torpor, a similar phenomenon that is characterized by a transient reduction in body temperature usually tied to the circadian rhythm and lowered food availability (Ruf \& Geiser, 2015). This is in contrast with hibernation, which is 
typically characterized by weeks of dormancy interspersed with short periods of arousal and lasts over multiple months during the colder periods of the year. Hibernation and daily torpor in mammals is common and distributed throughout most major mammalian taxa including marsupials and even non-therian mammals (monotremes), implying this mechanism may be as ancient as mammals themselves (Faherty, Villanueva-Cañas, Klopfer, Albà, \& Yoder, 2016; Geiser, 1994; Nicol, 2017).

In the Richardson's ground squirrel (Urocitellus richardsonii), the species studied in this thesis, hibernation occurs for the majority of the year and can last in some case up to 9 months. Entrance into torpor varies depending on age and sex, typically juvenile males enter torpor at the latest time (Wang, 1979). Males emerge slightly earlier from hibernation sometime around midMarch to establish territory. After the mating season is complete, the animals will feed extensively to rapidly accumulate fat stores to prepare for the next entrance into hibernation. The initial trigger for the onset of torpor is not entirely obvious as there may be multiple seasonal cues at play. The initiation of torpor is generally not driven primarily by the change in photoperiod during the autumn, as was originally postulated (Körtner \& Geiser, 2000). This is likely true for $U$. richardsonii since for this species the start of the hibernation season may occur as early as mid-July, long before the day length has substantially shortened. Indeed, several studies have demonstrated that if marmots and ground squirrel species are placed in a laboratory environment with constant photoperiod and temperature, they will continue to follow feeding and weight gain patterns consistent with the wild (Kenagy, 1981; Ward \& Armitage, 1981). This implies that the circannual rhythms of hibernators are driven, at least in part, by some sort of endogenous clock and are not entirely dependent on external cues. The decrease in ambient temperature over the autumn time frame is also not a good predictor of entrance into hibernation. 
For example, Richardson's ground squirrels will often start hibernation in late summer or early autumn even though environmental temperatures are still around $20^{\circ} \mathrm{C}$ (Wang, 1979). This may suggest that the cycle may be based at least partly around the feeding status of the animal. This is consistent with many species, including other members of the squirrel family, that undergo daily torpor and drop their nocturnal body temperature when encountering caloric restriction (Munro, Thomas, \& Humphries, 2005; Pereira, Aines, \& Scheckter, 2002). Exit from hibernation is likely due to a variety of factors and is thought to be mediated by cues from the pineal gland and the release of large amounts of sex steroids to activate the reproductive system in preparation for the emergence of torpor and subsequent breeding (Hut, Dardente, \& Riede, 2014).

Hibernation in mammals is characterized by numerous metabolic and physiological changes. Leading up to the hibernating season animals acquire large lipid stores, sometimes as much as doubling their body weight, in order to survive without feeding during hibernation (Michener, 1998). This is crucial for the animal since during hibernation, carbohydrate catabolism is greatly reduced and the oxidation of fats takes precedence. This preferential consumption of lipid substrates over carbohydrates is evidenced by the shift in respiratory quotient from 1 to between 0.7-0.8 (Buck \& Barnes, 2000). During hibernation, the synthesis of ketone bodies (acetoacetate and $\beta$-hydroxybutyrate) to fuel tissues such as the skeletal muscle occurs in the liver mitochondria of the ground squirrel (Rauch \& Behrisch, 1980). The presence of ketone bodies causes reduction in the uptake of glucose in skeletal muscle tissue with a concentration of $1.4 \mathrm{mM}$ sufficient to elicit a $100 \%$ decrease in the uptake of glucose in the pectoralis muscle (Krilowicz, 1985). During hibernation, the vital signs of the animal are greatly slowed including heart rate $\left(1 / 30^{\text {th }}\right.$ the normal pace), breathing rate $\left(\mathrm{O}_{2}\right.$ consumption reduced to $1 \%$ of normal euthermic levels), and body temperature is lowered to ambient or near-ambient 
temperature (Wang \& Lee, 1996). It should be noted, however, that the reduction of body temperature is not a complete absence of thermoregulation, but rather a change in the internal set point of the body that the animal will defend. This temperature is commonly a few degrees above $0{ }^{\circ} \mathrm{C}$ but in at least one extreme example (the arctic ground squirrel, Urocitellus parryii), the core body temperature of the animal may decrease at times to as low as $-2.9^{\circ} \mathrm{C}$ with freeze-avoidance strategies employed to allow supercooling of its body fluids (Barnes, 1989; Lee, Kohl, Buck, \& Barnes, 2016). The reduction in body temperature is likely directly responsible for a great deal of the reduction in metabolic rate seen in hibernation since enzymes may not function optimally at these temperatures. However, analysis of the $\mathrm{Q}_{10}$ temperature coefficient of $\mathrm{O}_{2}$ consumption in hibernating and torpid animals demonstrated that this value is higher across the temperature range of $20-30{ }^{\circ} \mathrm{C}\left(\mathrm{Q}_{10}=4.1\right)$ than it is at a lower temperature range of $0.1-10{ }^{\circ} \mathrm{C}\left(\mathrm{Q}_{10}=2.85\right)$ (Geiser, 1988). The $\mathrm{Q}_{10}$ temperature coefficient is a measurement of the effects of temperature on the rate of a chemical reaction represented by the following equation where $\mathrm{R}$ represents the rate of a chemical reaction and $\mathrm{T}$ represents the temperature:

$$
\mathrm{Q}_{10}=\left(\mathrm{R}_{1} / \mathrm{R}_{2}\right)^{10^{\circ} \mathrm{C} /(\mathrm{T} 2-\mathrm{T} 1)}
$$

Because $\mathrm{Q}_{10}$ of $\mathrm{O}_{2}$ consumption is greater at higher body temperatures than it is at lower temperatures, this implies that during entrance into torpor animals employ active metabolic restrictions and do not rely solely on the decline in body temperature in order to slow their metabolism.

Many of the physiological changes occurring during hibernation are known to be ultimately mediated via changes in the hormonal profile of the animal. This is especially true for the transition to a lipid-based metabolism. Because feeding stops during hibernation, ground 
squirrels have very low levels of circulating insulin, reaching their lowest levels by mid-winter (Bauman, Meryn, \& Florant, 1987; Carey, Andrews, \& Martin, 2003). Despite the low levels of circulating insulin, glucagon levels remain relatively constant as compared to euthermic animals (Bauman et al., 1987). Because glucagon is maintained and insulin secretion is suppressed the actions of glucagon predominate, specifically including the induction of lipolysis (Habegger et al., 2010). Glucagon activates protein kinase A (PKA) signaling in white adipose tissue (WAT) that in turn phosphorylates hormone sensitive lipase (HSL) to activate lipolysis and the transport of fatty acids through the blood (Moreau-Hamsany, Castex, Hoo-Paris, Kacemi, \& Suiter, 1988). Additionally, during the winter and spring, mRNA levels of HSL and lipoprotein lipase were found to be substantially elevated in WAT of hibernating marmots as compared to the fall and summer time periods (Wilson, Deeb, \& Florant, 1992). This study also demonstrated a strong reduction in fatty acid synthase mRNA levels during this same time period. Taken together this demonstrates a strong preference for the consumption of lipids over carbohydrates during hibernation.

Despite the reduced body temperature and dormant state afforded by the torpor response that enables a significant reduction in energy usage, most mammalian hibernators undergo periodic arousals from torpor at multiple points during the winter. The length between bouts of arousal is generally related to the body temperature of the torpid animal with lowest body temperatures being associated with the greatest length of time between arousals. In $U$. richardsonii, the maximum time between arousals occurs during the coldest periods of the winter when body temperature can fall to between $2-3^{\circ} \mathrm{C}$ and the time between arousals can reach approximately 20 days (Wang, 1979). Despite the ubiquity of interbout arousals in hibernation physiology, the purpose served by this rewarming back to euthermic temperature continues to be 
a topic of much speculation. It has been estimated that in one species, (the arctic ground squirrel, U. parryii) around $86 \%$ of energy expenditure during the hibernation season is consumed by these arousals, so they are not trivial and most likely serve a critical function for the survival of the animal (Karpovich, Tøien, Buck, \& Barnes, 2009). Although interbout arousals are reported in most mammalian hibernators, at least one species of tenrec, a family of small-bodied afrotherians native to Madagascar, are a notable exception to this rule and have been observed to undergo hibernation for as long as 9 months without any interbout arousals (Lovegrove, Lobban, \& Levesque, 2014). One popular theory regarding the significance of interbout arousals posits that torpor itself does not have the restorative properties of sleep and therefore the hibernator needs to emerge from torpor in order to have a proper sleep cycle. This is supported by evidence that demonstrates increased slow-wave sleep in animals sleeping during arousal periods which is consistent with what is seen in sleep deprived euthermic animals (Larkin \& Heller, 1999). This implies that there is a restorative quality to brain function during sleep that is absent during the torpor phase. It is interesting to note that afrotherians are notable for unusual sleep activity often displaying very little REM sleep. One such example is the rock hyrax (Procavia capensis) that has the lowest percentage of time recorded in REM sleep for any mammal (Gravett, Bhagwandin, Lyamin, Siegel, \& Manger, 2012). Additionally, the African elephant, another afrotherian, has one of the lowest known daily amount of sleep of any mammal (around $2 \mathrm{~h}$ ) and will often sleep standing up which may possibly limit the amount of time animals can spend in REM sleep which is typically characterized by low muscle tone (Gravett et al., 2017). Regardless of the reason for the hibernators to engage in interbout arousals, the fact that torpor represents a cyclical phenomenon suggests that changes in the metabolism of the animal must be readily reversible to cope with the rapidly changing metabolic demands of the torpor arousal cycle. 


\subsection{Metabolic Regulation}

Metabolic regulation is a complex phenomenon that can be conceptualized at a variety of different interlinked levels all of which are implicated in hibernation. One of the primary levels of regulation is at the level of the transcription of metabolically significant genes into mRNA. Typically, although not always, an increase in the production of a particular mRNA transcript corresponds to an increase in the downstream translation of the protein product (Liu, Beyer, \& Aebersold, 2016). Several different factors influence the transcription of genes into mRNAs. On a global level, the expression level of RNA polymerase can directly influence the overall rate of transcription (Ali \& Choubey, 2019). One recent study documented how transcription at the level of a single cell is usually done in controlled 'bursts', the duration and periodicity of these bursts affecting the overall rate of transcription (Bartman et al., 2019). While the total mRNA content in ground squirrels over hibernation appears to be relatively constant, the prioritization of mRNAs with internal ribosomal entry sites (IRES) during torpor can allow for more targeted translation of mRNAs that are critical for survival during torpor (Tessier \& Storey, 2014). Transcriptomic analysis of the liver mRNAs of hibernating 13-lined ground squirrels has suggested the importance of improved mRNA stability during bouts of torpor, despite the lack of transcription during this time, in preparation for the euthermic interbout arousals when these will be needed to reactivate metabolism (Gillen et al., 2021). While overall mRNA levels tend to remain relatively constant, analysis of the function of the mRNAs in this transcriptomic study demonstrated a decrease in those associated with metabolism (logical considering the reduction in metabolic rate) and an increase in those associated with stress response pathways (Gillen et al., 2021). By comparison, transcriptomic analysis of the skeletal muscle of the 13-lined ground squirrel has demonstrated evidence for increased oxidative capacity during hibernation, with 
particular emphasis on higher mRNA content for lipid catabolism such as those coding for the enzymes needed for $\beta$-oxidation of fatty acids as well as fatty acid transport to the mitochondria and peroxisomes (Vermillion, Anderson, Hampton, \& Andrews, 2015). Regulating transcript abundance are micro-RNAs, that can bind to mRNA to facilitate their degradation. Micro-RNAs play an important role in ground squirrel tissues during hibernation as a set of these targeting metabolism-promoting genes are found upregulated during torpor (Wu et al. 2016). Additionally, the overall level of genome methylation, both at the level of histones and of methylated cytosine residues on DNA, can inhibit transcription. Hibernation-specific protein 27 (HP-27), for example, was found to be regulated in liver of a chipmunk species through differential $\mathrm{CpG}$ promoter methylation (Fujii et al., 2006). A highly targeted approach to transcriptional regulation involves expression and function of transcription factors that are often under the control of various kinase-signaling pathways. In the suprachiasmatic nucleus of the brain, responsible for regulating much of the body's circadian rhythms, ground squirrels showed increased levels of the c-Fos transcription factor subunit associated with torpor as opposed to summer euthermia (Bratincsák et al., 2007).

Control of metabolism at the enzymatic level has been classically thought as predominantly occurring at specific regulatory steps that are subject to regulation through feedback inhibition or alterations to the structure/function of the enzyme. In this model of metabolic regulation, the rate-limiting steps as well as those that are irreversible under cellular conditions in the pathways are commonly thought to be the sole points of metabolic regulation (Rognstad, 1979). More recently, this model has been challenged as being overly simplistic and increasingly regulation of pathways is conceptualized as distributed across numerous points throughout the pathway described through Metabolic Control Analysis (MCA) mathematical modelling 
(Moreno- Sánchez, Saavedra, Rodríguez-Enríquez, \& Olín-Sandoval, 2008). The insufficiencies

of the single rate-limiting step model first became apparent when attempts to increase the output of metabolic pathways through overexpression of the rate-limiting enzyme tended to have a limited efficacy. While it may be that some steps are subjected to tighter regulation than others, the complexity of metabolic control over a pathway is more complicated than controlling a single step since metabolism typically does not consist of linear pathways, but rather many pathways that can be diverted along their course in competing reactions. This means that while some enzymes are likely to have a greater degree of significance in terms of overall control over a pathway, the cell also exerts a certain degree of control over numerous steps in pathways in a concerted effort to bring about regulation. For example, lactate dehydrogenase (LDH), has been characterized as having profound functional changes in in a number of different species undergoing metabolic rate depression to enable metabolic fine-tuning despite being classically thought of as a non-regulatory enzyme whose activity simply responds to alterations in substrate concentration (Storey, 2016). For this reason, the enzymes studied in this thesis should not be thought of as the sole mediators of the metabolic changes that occur in response to hibernation, but rather function in concert with other steps in the pathways to help bring about the overall changes to the metabolic rate.

Post-translational modifications (PTMs) of proteins, including phosphorylation, are an important element of metabolic regulation as they allow for rapid and often reversible control of critical metabolic enzymes. While control over the expression levels of enzymes in tissues of animals is still important, the energetic cost of translating additional protein and proteolysis of existing protein can be prohibitive, particularly when considering the cyclical metabolic stress that is imposed by frequent bouts of torpor-arousal. PTMs are likely an underappreciated mode 
of metabolic control owing to a few factors. The staggering diversity of PTMs that are found naturally in the cell presents a particular challenge in trying to assess the role of PTMs in vivo. Protein phosphorylation has been extensively characterized to the point where in silico tools have been developed for prediction of potential phosphorylation sites given a primary amino acid sequence as well as to suggest the relevant protein kinase involved, but there are numerous of other PTMs that are not as well characterized (Blom, Gammeltoft, \& Brunak, 1999; Blom, Sicheritz-Pontén, Gupta, Gammeltoft, \& Brunak, 2004). To date more than 200 types of PTMs have been documented on proteins in nature, providing enormous diversity in the way that proteins may be functionally altered (Duan \& Walther, 2015). In many cases, it is not even immediately clear if the addition of the PTM is achieved by an enzymatic or non-enzymatic mode of action (Harmel \& Fiedler, 2018). The multitude of possible PTMs can make it difficult to predict which ones are strong candidates in being relevant in the function of any given protein. Understanding the compartmentalized nature of the cell can help with this as some proteins are localized to specific subcellular locations and are more likely to be tagged with specific PTMs. For example, it is understood that in the mitochondria the addition of acyl groups via nonenzymatic means is common due to mitochondria functioning as a hub for the processing of various acyl CoA derivatives associated with fatty acid oxidation and the citric acid cycle (CAC) (Hirschey, Shimazu, Huang, Schwer, \& Verdin, 2011; Hong et al., 2016). Additionally, the interpretation of differing PTM patterns is not as obvious and as easy to understand as a change in gene expression. An increase in the phosphorylation of a protein for example could indicate an increase or a decrease in its activity. 


\subsection{Enzymatic Regulation in the Richardson's Ground Squirrel during Hibernation}

Numerous studies have already focused on understanding the metabolic regulation of hibernation in Richardson's ground squirrels at the enzymatic level. Given the pronounced reduction in glycolysis and a shift to fatty acid consumption instead during hibernation, many of these studies have focused on the regulation of glycolysis. For example, hexokinase (HK), the enzyme committing glucose to glycolysis, was shown to have a lower maximal activity in the muscle of hibernating squirrels and also exhibited reduced affinity for ATP $\left(80 \%\right.$ higher $\left.K_{m}\right)$ (Abnous \& Storey, 2008). This study also demonstrated that stimulation of AMP-dependent protein kinase reversed these effects on the hibernating enzyme but had no effect on HK from euthermic control animals. This contrasted with pyruvate kinase (PK) that was more phosphorylated in the muscle of hibernating animals, but exhibited lowered substrate affinity for phosphoenolpyruvate (Bell \& Storey, 2018). Differences in phosphorylation patterns despite similarities in functional changes during hibernation suggest that mechanisms governing the phosphorylation and regulation of these glycolytic enzymes vary and that likely multiple kinases are influenced by the effects of hibernation. In addition, lower maximal activity (but without change in substrate affinity) of the liver isoform of PK showed that regulation over the activity of these key glycolytic enzymes likely follows tissue specific patterns (Smolinski, Green, \& Storey, 2020).

Apart from glycolysis, the regulation of several other enzymes has been investigated in Richardson's ground squirrel. Creatine kinase (CK), the enzyme responsible for catalyzing the formation of phosphocreatine, was found to be $20 \%$ lower in abundance and activity during torpor and also showed reduced mRNA expression by $70 \%$. Phosphocreatine has an important role in muscle physiology by acting as an energy buffer to produce ATP rapidly during exercise 
and therefore a reduction in $\mathrm{CK}$ activity makes sense during the inactive state of hibernation (Abnous \& Storey, 2007). Similarly, AMP deaminase (AMPD), an enzyme that aids maintenance of total adenylate pools in muscle tissue was noted to be reduced in activity by as much as $84 \%$ during hibernation in this species. Stimulation of protein phosphatases led to reduced activity of AMPD in euthermic tissues, suggesting that phosphorylation was responsible for elevated AMPD activity (Abnous \& Storey, 2021). Analysis of glycerol-3-phosphate dehydrogenase in the muscle of hibernating Richardson's ground squirrels showed increased affinity for glycerol-3-phosphate (a product of triglyceride breakdown) at low temperature (5 ${ }^{\circ} \mathrm{C}$ ), suggesting that this mechanism may help to produce the glycolytic intermediate dihydroxyacetone phosphate which could in theory be utilized in gluconeogenesis (Ruberto, Logan, \& Storey, 2019). Use of gluconeogenesis to restore carbohydrate supplies has been suggested to occur during interbout arousals in ground squirrel hibernation (Galster \& Morrison, 1975).

\subsection{Citric Acid Cycle}

The citric acid cycle (CAC), also known as the Krebs cycle or tricarboxylic acid cycle, is a central metabolic hub that exists in all aerobic life on earth and is crucial for connecting catabolic and anabolic reactions. Critically, it is necessary in oxidizing the end products of glycolysis (pyruvate) and $\beta$-oxidation of fatty acids (acetyl coenzyme A (CoA)) to support oxidative phosphorylation and generate ATP in mitochondria (Fig. 1.1.). In the glycolytic pathway, glucose is oxidized to pyruvate that is then transported into mitochondria and oxidized by pyruvate dehydrogenase to form acetyl-CoA. Acetyl-CoA is an important intermediate in many reactions and in particular is the main substrate that feeds the CAC (Krebs, 1940). During hibernation in ground squirrels, glycolysis is greatly reduced and instead most of the energy 
budget is maintained via catabolism of triglycerides that were accumulated during the summer, again with the production of acetyl-CoA that is processed by the CAC. The CAC is important in energy metabolism by generating reduced cofactors $\left(\mathrm{NADH}\right.$ and $\left.\mathrm{FADH}_{2}\right)$ that are needed to run the electron transport chain (ETC) in the mitochondria. In this series of reductions, $\mathrm{H}^{+}$ions are conveyed to the intermembrane space where they form a proton gradient that subsequently drives ATP synthesis (Mitchell, 1961). This means that the CAC is an aerobic process since the final electron acceptor of the ETC is oxygen.

While it is commonly referred to as a cycle, many parts of the CAC do not necessarily turn in one direction but are bidirectional in living cells. Many of the intermediates of the CAC can be shunted out of the cycle and used in other processes such as amino acid or cholesterol synthesis (Berg, Tymoczko, \& Stryer, 2002; Feng, Zhang, Xu, \& Shen, 2020). This means that the irreversible steps of the $\mathrm{CAC}$ are more tightly regulated since they commit a substrate to a more specific metabolic fate. In the CAC there are only two irreversible steps; these are the reactions mediated by citrate synthase (CS) and the $\alpha$-ketoglutarate dehydrogenase complex (KGDC). The irreversibility of these reactions is a consequence of the highly negative change in Gibb's free energy attendant with the reaction under cellular conditions $(\Delta G=-37.66 \mathrm{~kJ} / \mathrm{mol}$ for KGDC and -36.60 kJ/mol for CS) (Li, Wu, Qi, \& Beard, 2011). Accordingly these steps are often subjected to feedback inhibition by the end products of the CAC such as NADH which inhibits CS (Lee, Son, \& Kim, 2019).

\subsection{Hypothesis}

The research contained herein seeks to address two main hypotheses. The first is that given the need for substantial metabolic suppression during hibernation to conserve fuel supplies, the key regulatory enzymes within the $\mathrm{CAC}$ and also those involved in providing 
substrates into this pathway will be regulated in the muscle tissue of the Richardson's ground squirrel to facilitate sparing of valuable fuel resources. This regulation will not likely take the form of complete inactivation since, even during hibernation, basal levels of activity are required in order to catabolize the lipid fuel that is predominantly utilized during hibernation but will instead exist as more moderate controls over enzyme activity.

Secondly, the nature of this regulation will likely occur at the level of the enzymes themselves and not by transcriptional or translational controls. Instead, the use of alternate PTM patterns in hibernating animals will help to serve as a metabolic switch. This seems likely given the need for animals to warm up during brief interbout arousals, strongly increasing metabolic rate, thereby making control in this manner particularly attractive from an efficiency perspective.

\subsection{Objectives}

Understanding the nature of metabolic regulation during mammalian hibernation necessitates learning about how the functional parameters of key regulatory enzymes are altered over the torpor/arousal phases of hibernation. While previous research on this species has often focused on the regulation of the glycolytic pathway, the research reported in this thesis investigates the role of enzymatic regulation of the CAC. This pathway must be an important site of metabolic regulation in hibernation since the $\mathrm{CAC}$ is central to integrating metabolic fuels from various upstream sources including carbohydrates, lipid, and protein. The research reported here examined the role of both enzymes within the CAC that serve as important rate-limiting or irreversible regulatory controls over the $\mathrm{CAC}$ and enzymes that are responsible for ferrying substrates into the CAC. 


\section{Objective 1: Pyruvate Dehydrogenase Regulation}

The pyruvate dehydrogenase complex (PDC) is a large multi-enzymatic complex composed of three repeated catalytic subunits and functions to link glycolysis to the CAC by oxidizing pyruvate to acetyl-CoA in the mitochondria. The regulation of this complex is wellconserved across all vertebrates and is understood to be regulation via the use of phosphorylation at three critical residues on the E1 or pyruvate dehydrogenase (PDH) subunit. In Chapter 2, the relative activity of the PDC is examined in muscle and liver tissues from euthermic versus hibernating ground squirrels and the phosphorylation status of the three enzymes is also evaluated. Additional consideration of the contributions made by the relevant PDH kinases (PDKs) is given to determine what roles they play during hibernation.

\section{Objective 2: Citrate Synthase Regulation}

Citrate synthase (CS) is the first step of the CAC and catalyzes the condensation reaction linking an acetyl group carried by acetyl-CoA to oxaloacetate (OAA). In Chapter 3, CS is purified from skeletal muscle of hibernating and euthermic ground squirrels and the kinetic parameters of this enzyme are measured and compared. The relative levels of various PTMs were also probed using immunoblotting to investigate mechanisms by which the activity of citrate synthase may be regulated during hibernation.

\section{Objective 3: $\alpha$-Ketoglutarate Dehydrogenase Regulation}

The $\alpha$-ketoglutarate dehydrogenase complex (KGDC) is another multi-enzymatic complex homologous to the PDC and catalyzes an irreversible reaction within the CAC. In 
Chapter 4, the functional properties of KGDC are explored with regard to the affinity for substrates and activity levels between the euthermia and hibernation. Analysis of the PTM status through immunoblotting is also carried out on all three subunits of the complex following immunoprecipitation to look for trends occurring during hibernation.

\section{Objective 4: Glutamate Dehydrogenase Regulation}

Glutamate dehydrogenase serves as an important link between amino acid catabolism and the CAC by producing $\alpha$-ketoglutarate from glutamate (a common intermediate in amino acid catabolism). In Chapter 5, the functional properties of glutamate dehydrogenase from euthermic and hibernating Richardson's ground squirrels are investigated to assess the regulation at this locus. Finally, the PTM status of GDH is assessed for the purified hibernating versus euthermic enzyme to investigate trends in the regulation of GDH. 


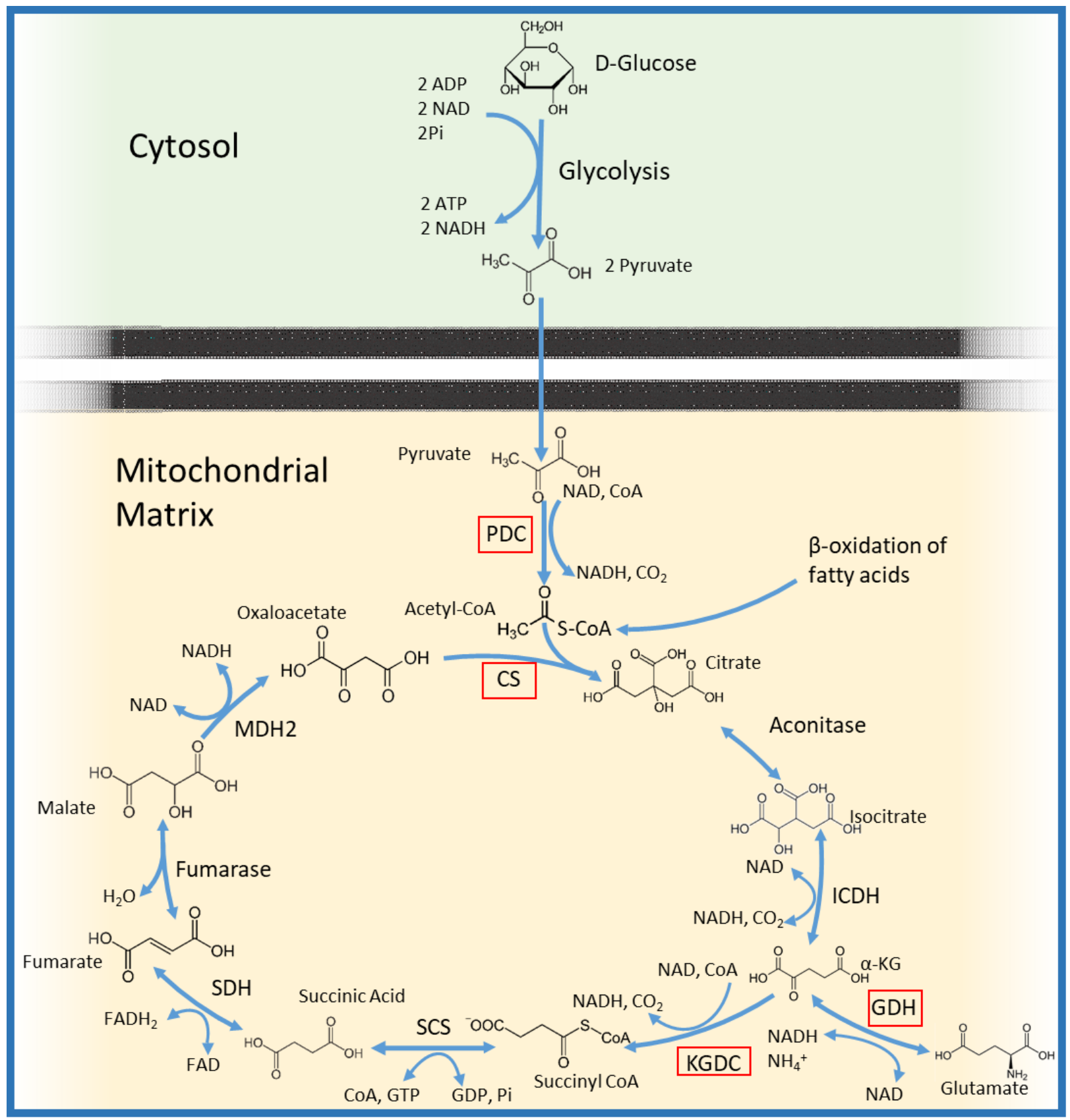

Fig. 1.1. Schematic diagram demonstrating the steps and relationship to central metabolism of the citric acid cycle. Abbreviations are as follows; ADP (adenosine diphosphate), NAD/NADH (nicotinamide adenine dinucleotide oxidized/reduced), Pi (inorganic phosphate), PDC (pyruvate dehydrogenase complex), CoA (coenzyme A), ICDH (isocitrate dehydrogenase), $\alpha-\mathrm{KG}(\alpha-$ ketoglutarate dehydrogenase), GDH (glutamate dehydrogenase), KGDC ( $\alpha$-ketoglutarate dehydrogenase complex), SCS (succinyl CoA synthetase), FAD/FADH2 (Flavin adenine dinucleotide oxidized/reduced), $\mathrm{MDH} 2$ (malate dehydrogenase, mitochondrial), CS (citrate synthase). 


\section{Chapter 1 References}

Abnous, K., \& Storey, K. B. (2007). Regulation of skeletal muscle creatine kinase from a hibernating mammal. Archives of Biochemistry and Biophysics, 467(1), 10-19. https://doi.org/10.1016/j.abb.2007.07.025

Abnous, K., \& Storey, K. B. (2008). Skeletal muscle hexokinase: Regulation in mammalian hibernation. Molecular and Cellular Biochemistry, 319(1-2), 41-50. https://doi.org/10.1007/s11010-008-9875-5

Abnous, K., \& Storey, K. B. (2021). 5'-Adenosine monophosphate deaminase regulation in ground squirrels during hibernation. Comparative Biochemistry and Physiology Part - B: Biochemistry and Molecular Biology, 253, 110543. https://doi.org/10.1016/j.cbpb.2020.110543

Ali, M. Z., \& Choubey, S. (2019). Decoding the grammar of transcriptional regulation from RNA polymerase measurements: Models and their applications. Physical Biology, 16(6), 061001. https://doi.org/10.1088/1478-3975/ab45bf

Barnes, B. M. (1989). Freeze avoidance in a mammal: Body temperatures below $0{ }^{\circ} \mathrm{C}$ in an arctic hibernator. Science, 244(4912), 1593-1595. https://doi.org/10.1126/science.2740905

Bar-On, Y. M., Phillips, R., \& Milo, R. (2018). The biomass distribution on Earth. Proceedings of the National Academy of Sciences of the United States of America, 115(25), 6506-6511. https://doi.org/10.1073/pnas.1711842115

Bartman, C. R., Hamagami, N., Keller, C. A., Giardine, B., Hardison, R. C., Blobel, G. A., \& Raj, A. (2019). Transcriptional burst initiation and polymerase pause release are key control points of transcriptional regulation. Molecular Cell, 73(3), 519-532.e4. https://doi.org/10.1016/j.molcel.2018.11.004

Bauman, W. A., Meryn, S., \& Florant, G. L. (1987). Pancreatic hormones in the nonhibernating and hibernating golden mantled ground squirrel. Comparative Biochemistry and Physiology -- Part A: Physiology, 86(2), 241-244. https://doi.org/10.1016/0300-9629(87)90324-0

Bell, R. A. V., \& Storey, K. B. (2018). Purification and characterization of skeletal muscle pyruvate kinase from the hibernating ground squirrel, Urocitellus richardsonii: potential regulation by posttranslational modification during torpor. Molecular and Cellular Biochemistry, 442(1-2), 47-58. https://doi.org/10.1007/s11010-017-3192-9

Bennett, A., \& Ruben, J. (1979). Endothermy and activity in vertebrates. Science, 206(4419), 649-654. https://doi.org/10.1126/science.493968

Berg, J. M., Tymoczko, J. L., \& Stryer, L. (2002). Amino acids are made from intermediates of the citric acid cycle and other major pathways. Retrieved from https://www.ncbi.nlm.nih.gov/books/NBK22459/ 
Blom, N., Gammeltoft, S., \& Brunak, S. (1999). Sequence and structure-based prediction of eukaryotic protein phosphorylation sites. Journal of Molecular Biology, 294(5), 1351-1362. https://doi.org/10.1006/jmbi.1999.3310

Blom, N., Sicheritz-Pontén, T., Gupta, R., Gammeltoft, S., \& Brunak, S. (2004). Prediction of post-translational glycosylation and phosphorylation of proteins from the amino acid sequence. Proteomics. https://doi.org/10.1002/pmic.200300771

Brashears, J. A., Hoffman, T. C. M., \& DeNardo, D. F. (2017). Modeling the costs and benefits associated with the evolution of endothermy using a robotic python. The Journal of Experimental Biology, 220(13), 2409-2417. https://doi.org/10.1242/jeb.151886

Bratincsák, A., McMullen, D., Miyake, S., Tóth, Z. E., Hallenbeck, J. M., \& Palkovits, M. (2007). Spatial and temporal activation of brain regions in hibernation: c-fos expression during the hibernation bout in thirteen-lined ground squirrel. Journal of Comparative Neurology, 505(4), 443-458. https://doi.org/10.1002/cne.21507

Bromley, R. G., \& Jarvis, R. L. (1993). The energetics of migration and reproduction of dusky Canada geese. The Condor, 95(1), 193-210. https://doi.org/10.2307/1369400

Buck, C. L., \& Barnes, B. M. (2000). Effects of ambient temperature on metabolic rate, respiratory quotient, and torpor in an arctic hibernator. American Journal of Physiology Regulatory Integrative and Comparative Physiology, 279, R255-R262. https://doi.org/10.1152/ajpregu.2000.279.1.r255

Carey, H. V., Andrews, M. T., \& Martin, S. L. (2003). Mammalian hibernation: cellular and molecular responses to depressed metabolism and low temperature. Physiological Reviews, 83(4), 1153-1181. https://doi.org/10.1152/physrev.00008.2003

Costanzo, J. P. (2019). Overwintering adaptations and extreme freeze tolerance in a subarctic population of the wood frog, Rana sylvatica. Journal of Comparative Physiology B: Biochemical, Systemic, and Environmental Physiology. https://doi.org/10.1007/s00360-0181189-7

Costanzo, J. P., Reynolds, A. M., do Amaral, M. C. F., Rosendale, A. J., \& Lee, R. E. (2015). Cryoprotectants and extreme freeze tolerance in a subarctic population of the wood frog. PloS One, 10(2), e0117234. https://doi.org/10.1371/journal.pone.0117234

Duan, G., \& Walther, D. (2015). The roles of post-translational modifications in the context of protein interaction networks. PLoS Computational Biology, 11(2). https://doi.org/10.1371/journal.pcbi.1004049

Faherty, S. L., Villanueva-Cañas, J., Klopfer, P. H., Albà, M. M., \& Yoder, A. D. (2016). Gene expression profiling in the hibernating primate, Cheirogaleus medius. Genome Biology and Evolution, 8(8). https://doi.org/10.1093/gbe/evw163 
Feng, X., Zhang, L., Xu, S., \& Shen, A. (2020). ATP-citrate lyase (ACLY) in lipid metabolism and atherosclerosis: An updated review. Progress in Lipid Research, 77. https://doi.org/10.1016/j.plipres.2019.101006

Fijn, R. C., Hiemstra, D., Phillips, R. A., \& Van Der Winden, J. (2013). Arctic Terns Sterna paradisaea from the Netherlands migrate record distances across three oceans to Wilkes Land, East Antarctica. Ardea, 101(1), 3-12. https://doi.org/10.5253/078.101.0102

Fleming, T. H. (2019). Bat migration. In Encyclopedia of Animal Behavior (pp. 605-610). https://doi.org/10.1016/B978-0-12-809633-8.20764-4

Fristoe, T. S., Burger, J. R., Balk, M. A., Khaliq, I., Hof, C., \& Brown, J. H. (2015). Metabolic heat production and thermal conductance are mass-independent adaptations to thermal environment in birds and mammals. Proceedings of the National Academy of Sciences, 112(52), 15934-15939. https://doi.org/10.1073/pnas.1521662112

Fujii, G., Nakamura, Y., Tsukamoto, D., Ito, M., Shiba, T., \& Takamatsu, N. (2006). CpG methylation at the USF-binding site is important for the liver-specific transcription of the chipmunk HP-27 gene. Biochemical Journal, 395(1), 203-209. https://doi.org/10.1042/BJ20051802

Galster, W., \& Morrison, P. R. (1975). Gluconeogenesis in arctic ground squirrels between periods of hibernation. American Journal of Physiology, 228(1), 325-330. https://doi.org/10.1152/ajplegacy.1975.228.1.325

Geiser, F. (1988). Reduction of metabolism during hibernation and daily torpor in mammals and birds: temperature effect or physiological inhibition? Journal of Comparative Physiology B, 158(1), 25-37. https://doi.org/10.1007/BF00692726

Geiser, F. (1994). Hibernation and daily torpor in Marsupials: A review. Australian Journal of Zoology, 42(1), 1-16. https://doi.org/10.1071/ZO9940001

Geiser, F., \& Ruf, T. (1995). Hibernation versus daily torpor in mammals and birds Physiological variables and classification of torpor patterns. Physiological Zoology, 68(NOVEMBER), 935-966. https://doi.org/10.2307/30163788

Gillen, A. E., Fu, R., Riemondy, K. A., Jager, J., Fang, B., Lazar, M. A., \& Martin, S. L. (2021). Liver transcriptome dynamics during hibernation are shaped by a shifting balance between transcription and RNA stability. Frontiers in Physiology, 12, https://doi.org/10.3389/fphys.2021.662132

Gravett, N., Bhagwandin, A., Lyamin, O. I., Siegel, J. M., \& Manger, P. R. (2012). Sleep in the rock hyrax, procavia capensis. Brain, Behavior and Evolution, 79(3), 155-169. https://doi.org/10.1159/000335342

Gravett, N., Bhagwandin, A., Sutcliffe, R., Landen, K., Chase, M. J., Lyamin, O. I., ... Manger, P. R. (2017). Inactivity/sleep in two wild free-roaming African elephant matriarchs - Does 
large body size make elephants the shortest mammalian sleepers? PLoS ONE, 12(3). https://doi.org/10.1371/journal.pone.0171903

Habegger, K. M., Heppner, K. M., Geary, N., Bartness, T. J., DiMarchi, R., \& Tschöp, M. H. (2010). The metabolic actions of glucagon revisited. Nature Reviews Endocrinology, 6(12), 689-697. https://doi.org/10.1038/nrendo.2010.187

Harmel, R., \& Fiedler, D. (2018). Features and regulation of non-enzymatic post-translational modifications. Nature Chemical Biology, 14(3), 244-252. https://doi.org/10.1038/nchembio.2575

Hirschey, M. D., Shimazu, T., Huang, J. Y., Schwer, B., \& Verdin, E. (2011). SIRT3 regulates mitochondrial protein acetylation and intermediary metabolism. Cold Spring Harbor Symposia on Quantitative Biology, 76, 267-277. https://doi.org/10.1101/sqb.2011.76.010850

Hong, S. Y., Ng, L. T., Ng, L. F., Inoue, T., Tolwinski, N. S., Hagen, T., \& Gruber, J. (2016). The role of mitochondrial non-enzymatic protein acylation in ageing. PLoS ONE, 11(12). https://doi.org/10.1371/journal.pone.0168752

Hut, R. A., Dardente, H., \& Riede, S. J. (2014). Seasonal timing: How does a hibernator know when to stop hibernating? Current Biology, 24(13), R602-R605. https://doi.org/10.1016/j.cub.2014.05.061

Jaeger, E. C. (1949). Further observations on the hibernation of the poor-will. The Condor, 51(3), 105-109. Retrieved from https://www.jstor.org/stable/pdf/1365104.pdf?refreqid=excelsior\%3Aad250ad8cfed35c470 $8 \mathrm{ff} 2 \mathrm{f} 99 \mathrm{fb} 7 \mathrm{f} 844$

Karpovich, S. A., Tøien, Ø., Buck, C. L., \& Barnes, B. M. (2009). Energetics of arousal episodes in hibernating arctic ground squirrels. Journal of Comparative Physiology B: Biochemical, Systemic, and Environmental Physiology, 179(6), 691-700. https://doi.org/10.1007/s00360009-0350-8

Kenagy, G. J. (1981). Effects of day length, temperature, and endogenous control on annual rhythms of reproduction and hibernation in chipmunks (Eutamias ssp.). Journal of Comparative Physiology $\square$ A, 141(3), 369-378. https://doi.org/10.1007/BF00609939

Körtner, G., \& Geiser, F. (2000). The temporal organization of daily torpor and hibernation: Circadian and circannual rhythms. Chronobiology International, 17(2), 103-128. https://doi.org/10.1081/CBI-100101036

Krebs, H. A. (1940). The citric acid cycle and the Szent-Györgyi cycle in pigeon breast muscle. Biochemical Journal, 34(5), 775-779. https://doi.org/10.1042/bj0340775

Krilowicz, B. (1985). Ketone body metabolism in a ground squirrel during hibernation and fasting. American Journal of Physiology - Regulatory Integrative and Comparative Physiology, 18(4), https://doi.org/10.1152/ajpregu.1985.249.4.R462 
Larkin, J. E., \& Heller, H. C. (1999). Sleep after arousal from hibernation is not homeostatically regulated. American Journal of Physiology - Regulatory Integrative and Comparative Physiology, 276(2 45-2). https://doi.org/10.1152/ajpregu.1999.276.2.r522

Lee, S. H., Son, H. F., \& Kim, K. J. (2019). Structural insights into the inhibition properties of archaeon citrate synthase from Metallosphaera sedula. PLoS ONE, 14(2), e0212807. https://doi.org/10.1371/journal.pone.0212807

Lee, T. N., Kohl, F., Buck, C. L., \& Barnes, B. M. (2016). Hibernation strategies and patterns in sympatric arctic species, the Alaska marmot and the arctic ground squirrel. Journal of Mammalogy, 97(1), 135-144. https://doi.org/10.1093/jmammal/gyv163

Li, X., Wu, F., Qi, F., \& Beard, D. A. (2011). A database of thermodynamic properties of the reactions of glycolysis, the tricarboxylic acid cycle, and the pentose phosphate pathway. Database, 2011. https://doi.org/10.1093/database/bar005

Liu, Y., Beyer, A., \& Aebersold, R. (2016). On the Dependency of Cellular Protein Levels on mRNA Abundance. Cell, 165(3), 535-550. https://doi.org/10.1016/j.cell.2016.03.014

Lovegrove, B. G., Lobban, K. D., \& Levesque, D. L. (2014). Mammal survival at the Cretaceous- Palaeogene boundary: Metabolic homeostasis in prolonged tropical hibernation in tenrecs. Proceedings of the Royal Society B: Biological Sciences, 281(1796). https://doi.org/10.1111/j.1574-6968.1991.tb04703.x-i1

Michener, G. R. (1998). Sexual differences in reproductive effort of Richardson's ground squirrels. Journal of Mammalogy, 79(1), 1-19. https://doi.org/10.2307/1382838

Mitchell, P. (1961). Coupling of phosphorylation to electron and hydrogen transfer by a chemiosmotic type of mechanism. Nature, 191(4784), 144-148. https://doi.org/10.1038/191144a0

Moreau-Hamsany, C., Castex, C., Hoo-Paris, R., Kacemi, N., \& Suiter, B. (1988). Hormonal control of lipolysis from the white adipose tissue of hibernating jerboa (Jaculus orientalis). Comparative Biochemistry and Physiology -- Part A: Physiology, 91(4), 665-669. https://doi.org/10.1016/0300-9629(88)90945-0

Munro, D., Thomas, D. W., \& Humphries, M. M. (2005). Torpor patterns of hibernating eastern chipmunks Tamias striatus vary in response to the size and fatty acid composition of food hoards. Journal of Animal Ecology, 74(4), 692-700. https://doi.org/10.1111/j.13652656.2005.00968.x

Nicol, S. C. (2017). Energy Homeostasis in Monotremes. Frontiers in Neuroscience, 11 (APR). https://doi.org/10.3389/fnins.2017.00195

Pereira, M. E., Aines, J., \& Scheckter, J. L. (2002). Tactics of heterothermy in eastern gray squirrels (Sciurus carolinensis). Journal of Mammalogy, 83(2), 467-477. https://doi.org/10.1644/1545-1542(2002)083<0467:TOHIEG >2.0.CO;2 
Pincheira-Donoso, D., Bauer, A. M., Meiri, S., \& Uetz, P. (2013). Global taxonomic diversity of living reptiles. PLoS ONE, 8(3), 1-10. https://doi.org/10.1371/journal.pone.0059741

Quirt, K. C., Blouin-Demers, G., Howes, B. J., \& Lougheed, S. C. (2006). Microhabitat selection of five-lined skinks in northern peripheral populations. Journal of Herpetology, 40(3), 335342. https://doi.org/10.1670/0022-1511(2006)40[335:MSOFSI]2.0.CO;2

Rauch, J., \& Behrisch, H. (1981). Ketone bodies: a source of energy during hibernation. Canadian Journal of Zoology, 59(5), 754-760. 10. https://doi.org/1139/z81-108

Rognstad, R. (1979). Rate-limiting steps in metabolic pathways. Journal of Biological Chemistry, 254(6), 1875-1878. https://doi.org/10.1016/s0021-9258(17)37738-4

Ruberto, A. A., Logan, S. M., \& Storey, K. B. (2019). Temperature and serine phosphorylation regulate glycerol-3-phosphate dehydrogenase in skeletal muscle of hibernating Richardson's ground squirrels. Biochemistry and Cell Biology, 97(2), 148-157. https://doi.org/10.1139/bcb-2018-0198

Ruf, T., \& Geiser, F. (2015). Daily torpor and hibernation in birds and mammals. Biological Reviews, 90(3), 891-926. https://doi.org/10.1111/brv.12137

Saleem, R., Al-attar, R., \& Storey, K. B. (2021). The activation of prosurvival pathways in Myotis lucifugus during torpor. Physiological and Biochemical Zoology, 94(3), 180-187. https://doi.org/10.1086/714219

Smolinski, M. B., Green, S. R., \& Storey, K. B. (2020). Characterizing the regulation of pyruvate kinase in response to hibernation in ground squirrel liver (Urocitellus richardsonii). Comparative Biochemistry and Physiology Part - B: Biochemistry and Molecular Biology, 248-249. https://doi.org/10.1016/j.cbpb.2020.110466

Storey, K. B. (2016) Comparative enzymology - new insights from studies of an "old" enzyme, lactate dehydrogenase. Comparative Biochemistry and Physiology Part B: Biochemistry and Molecular Biology, 199, 13-20. https://doi.org/10.1016/j.cbpb.2015.12.004

Storey, K. B., \& Storey, J. M. (2017). Molecular Physiology of Freeze Tolerance in Vertebrates. Physiological Reviews, 97(2), 623-665. https://doi.org/10.1152/physrev.00016.2016

Tessier, S. N., \& Storey, K. B. (2014). To be or not to be: the regulation of mRNA fate as a survival strategy during mammalian hibernation. Cell Stress and Chaperones, 19(6), 763776. https://doi.org/10.1007/s12192-014-0512-9

Tucker, V. A. (1970). Energetic cost of locomotion in animals. Comparative Biochemistry And Physiology, 34(4), 841-846. https://doi.org/10.1016/0010-406X(70)91006-6

Usher, M. B., \& Edwards, M. (1984). A dipteran from south of the Antarctic Circle: Belgica antarctica (Chironomidae) with a description of its larva. Biological Journal of the Linnean Society, 23(1), 19-31. https://doi.org/10.1111/j.1095-8312.1984.tb00803.x 
Vermillion, K. L., Anderson, K. J., Hampton, M., \& Andrews, M.T. (2015). Gene expression changes controlling distinct adaptations in the hear and skeletal muscle of a hibernating mammal. Physiological Genomics, 47(30), 58-74.

https://doi.org/10.1152/physiolgenomics.00108.2014

Wang, L. C. H. (1979). Time patterns and metabolic rates of natural torpor in the Richardson's ground squirrel. Canadian Journal of Zoology, 57(2), 149-155. https://doi.org/10.1139/z79012

Wang, L. C. H., \& Lee, T. F. (1996). Torpor and hibernation in mammals: Metabolic, physiological, and biochemical adaptations. Handbook of Physiology - Environmental Physiology, 507-532. https://doi.org/10.1002/cphy.cp040122

Ward, J. M., \& Armitage, K. B. (1981). Circannual rhythms of food consumption, body mass, and metabolism in yellow-bellied marmots. Comparative Biochemistry and Physiology -Part A: Physiology, 69(4), 621-626. https://doi.org/10.1016/0300-9629(81)90146-8

Wilson, B. E., Deeb, S., \& Florant, G. L. (1992). Seasonal changes in hormone-sensitive and lipoprotein lipase mRNA concentrations in marmot white adipose tissue. American Journal of Physiology - Regulatory Integrative and Comparative Physiology, 262(2 31-2). https://doi.org/10.1152/ajpregu.1992.262.2.r177

Woods, C. P., Czenze, Z. J., \& Brigham, R. M. (2019). The avian “hibernation” enigma: thermoregulatory patterns and roost choice of the common poorwill. Oecologia, 189(1), 4753. https://doi.org/10.1007/s00442-018-4306-0

Wu, C., Biggar, K.K., Luu, B. E., Szereszewski, K. E., \& Storey, K. B. (2016). Analysis of microRNA expression during the torpor-arousal cycle of a mammalian hibernator, the 13lined ground squirrel. Physiological Genomics, 48(6), 388-396.

https://doi.org/10.1152/physiolgenomics.00005.2016 


\section{Chapter 2: Hibernating PDH in Muscle vs. Liver}




\section{Functional and post-translational characterization of pyruvate dehydrogenase demonstrates repression of activity in the liver but not skeletal muscle of the Richardson's ground squirrel (Urocitellus richardsonii) during hibernation}

Stuart R. Green and Kenneth B. Storey

Institute of Biochemistry, Carleton University, 1125 Colonel By Drive, Ottawa ON, K1S 5B6, Canada

The following study has been published in the Journal of Thermal Biology

Green, S.R. and Storey, K.B. (2021) Functional and post-translational characterization of pyruvate dehydrogenase demonstrates repression of activity in the liver but not skeletal muscle of the Richardson's ground squirrel (Urocitellus richardsonii) during hibernation. Journal of Thermal Biology. 99. DOI: 10.1016/j.jtherbio.2021.102996 


\begin{abstract}
Hibernation consists of a series of physiological and biochemical alterations in an animal that allows for reduced body temperatures down to near ambient levels and substantial fuel conservation allowing it to survive on stored fat supplies accumulated during the summer. The Richardson's ground squirrel is one such hibernator that undergoes such changes for as long as 9 months of the year. This study examines the role of the regulation of the pyruvate dehydrogenase complex (PDC) during hibernation in the skeletal muscle and liver of the Richardson's ground squirrel. The current study demonstrates a great reduction in the activity of PDC in the hibernating liver, but not in the skeletal muscle. This was matched by a significant increase in the phosphorylation on a regulatory serine residue (S300) of the pyruvate dehydrogenase (PDH) E1 $\alpha$ subunit. Examining the expression patterns of the relevant kinases for PDH and the associated phosphatase demonstrated some unexpected results. Specifically, an increase in PDKs 1 and 2 and a decrease in PDK4 was noted in the skeletal muscle tissue in response to hibernation and no alterations in the expression patterns of any of these enzymes were noted in the liver. This suggests that alternative modes of regulation of the kinases may be at play in hibernation to bring about the observed effects. Taken together this study demonstrates that PDH regulatory responses differ markedly between tissues and emphasize the importance of inhibition of the complex in the liver during hibernation.
\end{abstract}

Keywords: PDH, hibernation, liver, skeletal muscle, metabolism, phosphorylation 


\section{Abbreviations}

CAC, Citric Acid Cycle; PDC, pyruvate dehydrogenase complex; PDH, pyruvate dehydrogenase (E1); PDK, pyruvate dehydrogenase kinase; PDP1, pyruvate dehydrogenase phosphatase catalytic subunit; PTM, post-translational modification

\subsection{Introduction}

In the winter, many animals employ dramatic changes to their behavior and physiology in order to cope with the reduction in the availability of nutrition and water. Hibernation is one of these adaptations and is a crucial survival strategy for many small mammalian species during the winter months. Hibernation constitutes a complex adaptation for winter survival that includes several alterations to the metabolic processes of the animal including a reduced set-point for the body temperature, often marginally above ambient temperature, and reduced vital sign activity such as lowered heart and breathing rate (Van Breukelen and Martin, 2015; Wang and Lee, 1996). This allows for the animal to greatly reduce its caloric consumption during the winter months and in turn for the animal's bodily functions to be sustained on stored lipids that were accumulated over the summer months. The reduction in metabolic rate can be partly explained by the decrease in body temperature leading to slowing of the biochemical processes of the hibernator; however, evidence suggests that active measures are also employed at the onset of torpor in order to lower the metabolic rate further. This is demonstrated by a much higher $\mathrm{Q}_{10}$ value between 20 and $30{ }^{\circ} \mathrm{C}\left(\mathrm{Q}_{10}=4.1\right)$ as opposed to the $\mathrm{Q}_{10}$ between 0.1 and $10{ }^{\circ} \mathrm{C}\left(\mathrm{Q}_{10}=\right.$ 2.85) measured by $\mathrm{O}_{2}$ consumption (Geiser, 1988). This strongly suggests that other mechanisms 
are at play in reducing the overall metabolic rate of hibernators and that these changes are likely at least in part due to regulation of central enzymes involved in fuel catabolism.

The Richardson's ground squirrel is a small fossorial rodent native to western North America with a range extending as far north as central Alberta. These animals are excellent model hibernators and have been studied for several decades owing to their phenomenal ability to survive the harsh Canadian winter (Wang, 1973). Central to this ability is the capacity of the squirrel to endure hibernation for up to 9 months of the year. To accomplish this, these animals double their body weight in fat stores during the summer and reduce their metabolic rates by $88 \%$ of their summer euthermic levels (Wang, 1979). This is accompanied by a decrease in body temperature down to a minimum around $2-3{ }^{\circ} \mathrm{C}$ during the coldest months of winter (Wang and Lee, 1996). These impressive physiological feats suggest that a remarkable degree of metabolic fine-tuning must occur during hibernation to meet the changing needs of the animal over the hibernation cycle.

To date much research has focused on the glycolytic capacity of this species, with several studies demonstrating reduction in the activity of key glycolytic enzymes in muscle and liver tissue during hibernation. Recent studies have demonstrated a reduction in pyruvate kinase (PK) activity in the liver (Smolinski et al., 2020) and a decrease in PK PEP affinity in muscle tissue associated with hibernation (Bell and Storey, 2018). Less however is understood regarding the regulation of mitochondrial respiration in the tissues of these animals during hibernation. A study by the authors has recently demonstrated a reduction in substrate affinity in the enzyme complex, $\alpha$-ketoglutarate dehydrogenase, governing a rate-limiting and irreversible step within the citric acid cycle of the muscle tissue in this animal (Green and Storey, 2020). This suggests that in 
addition to the reduction of upstream glycolytic processes the overall rate of the citric acid cycle, the central hub responsible for governing mitochondrial respiration, is likely slowed during hibernation to conserve further energy supplies from a variety of sources in the cell. This interpretation is supported by other authors who have observed that the mitochondrial respiration is reduced in the liver of a related ground squirrel species, the 13-lined ground squirrel (Ictidomys tridecemlineatus) during hibernation (Mathers and Staples, 2019). The present study sought to assess the functional parameters during hibernation of the regulatory step responsible for transferring the end product of glycolysis into the mitochondrial matrix; the pyruvate dehydrogenase complex (PDC).

The reaction catalyzed by the PDC is highly favourable $(\Delta \mathrm{G}=-39.26 \mathrm{~kJ} / \mathrm{mol})$ and is essentially irreversible under cellular conditions (Li et al., 2011). Additionally, the position of this complex in overall catabolic processes is such that it forms a critical bridge between the glycolytic pathway and the citric acid cycle (CAC). Taken together, this means that the PDC forms an important point of control over catabolic processes in the cell. The PDC is a large ( $\sim 9$ MDa in Homo sapiens) multi-enzyme complex that together catalyzes the oxidative decarboxylation of pyruvate to generate acetyl-CoA and NADH in the mitochondria (Patel et al., 2014). The complex is composed of multiple copies of three different proteins and its structure and function is analogous to two other keto-acid dehydrogenases, namely the $\alpha$-ketoglutarate and branched chain keto-acid dehydrogenase complexes. This family of complexes has three basic functional components, designated as E1, E2, and E3 or dihydrolipoamide dehydrogenase that is the same in all three complexes. Regulation over the PDC typically is thought to occur at the E1 pyruvate dehydrogenase (PDH) subunit of the complex (Holness and Sugden, 2003). The PDH itself is a heterotetramer composed of $2 \alpha$ and $2 \beta$ subunits. The E1 $\alpha$ subunit is responsible for 
the overall rate of reaction of the whole complex and is largely controlled through phosphorylation at 3 highly conserved serine residues (Lissens et al., 2000). These residues form such an important metabolic switch that they are conserved not only in most animal species but also similar mechanisms of inhibitory phosphorylation exist in plants and fungi as well (Gey et al., 2008; Weraduwage et al., 2016).

Phosphorylation at these residues promotes inhibition of the activity of the complex and is commonly associated with reduction in caloric availability (Korotchkina and Patel, 1995). The phosphorylation of these three sites is carried out by a family of 4 kinases, pyruvate dehydrogenase kinases (PDK), in mammals and is reversed through the activity of pyruvate dehydrogenase phosphatase 1 (PDP1) (Zhang et al., 2014). The activity of PDP1 is activated by the presence of $\mathrm{Ca}^{2+}$ ions in the mitochondrial matrix (Vassylyev and Symersky, 2007). PDK4 expression in human cells has been demonstrated to be responsive to insulin levels with decreased levels of insulin leading to increases in PDK4 expression (Huang et al., 2002). The presence of multiple isoforms of PDK has been proposed to be important in specifying which site is targeted for phosphorylation and is controlled through preferential expression of PDKs in a tissue dependent manner (Klyuyeva et al., 2019). While it is generally accepted that each of the canonical phosphorylation events at the three main sites is associated with decreases in activity, there has been some conjecture regarding the specific roles of each of these sites with regard to activity. The role of S232 for instance, has been associated with decreased stability of the protein complex and therefore can lead to lower abundance of the complex (Zhuang et al., 2019).

This current study evaluated the activity and phosphorylation state of the PDH in ground squirrel liver and muscle tissue from animals in the euthermic and hibernating state. This 
research was expanded to assess the relative levels of the 4 mammalian pyruvate kinase isoforms and PDP1. The results demonstrate that both tissues are not equally affected by the entrance into hibernation in terms of the function of PDH.

\subsection{Materials and Methods}

\subsubsection{Animal Treatments}

Male Richardson's ground squirrels, Urocitellus richardsonii, were captured near the Rocky Mountains in Alberta in the month of September and transported to the animal care facility at the University of Calgary. All 40 animals were initially housed individually in rat cages at $22{ }^{\circ} \mathrm{C}$ on a $10 \mathrm{~h}$ light to $14 \mathrm{~h}$ dark photoperiod. Animals were fed ad libitum for a period of 8 weeks before the animals were randomly divided into euthermic and hibernating groups. The 20 hibernating animals were moved to a cold room set to $4{ }^{\circ} \mathrm{C}$ on a photoperiod of $8 \mathrm{~h}$ light to $16 \mathrm{~h}$ dark. After $24 \mathrm{~h}$ under these conditions, the animals were withheld food (but not water) to initiate the hibernation response. The activity of the animals was monitored over this period for 1 week and any animals that did not enter into hibernation by the end of the week were removed from the experiment. Squirrels that were hibernating by the end of the week were held at $4{ }^{\circ} \mathrm{C}$ for two additional days after so that the minimum number of days of torpor was 2 days and the maximum was 7 days. Animals were within their first bout of torpor and were euthanized before the first interbout arousal. Euthermic animals were kept at $22{ }^{\circ} \mathrm{C}$ and remained fed during the experiments. Animals were sacrificed through decapitation and were dissected quickly to remove the liver and muscle tissues used in these experiments that were then immersed in liquid nitrogen and then transferred to $\mathrm{a}-80{ }^{\circ} \mathrm{C}$ freezer. The animal care and experimental regimen had gained approval by the university animal care committee before begun, following the Canadian Council on Animal Care guidelines. 


\subsubsection{Sequence Alignments}

To ensure that the kits used to probe the structural and functional properties used in this study would be effective in assaying PDH from a ground squirrel species, the sequence similarity to the intended target (human PDH) had to be assessed first. The sequence for PDH E1 $\alpha$ (the subunit responsible for regulation of the PDH complex and the one examined in assays) was compared in the closest related sequenced species of ground squirrel (the 13-lined ground squirrel, (Ictidomys tridecemlineatus). This was performed using the Clustal Omega Multiple Sequence Alignment Tool (Sievers et al., 2011). The sequences used in this alignment were as follows: Homo sapiens pyruvate dehydrogenase E1 component subunit alpha (NCBI Reference Sequence: NP_000275.1) and I. tridecemlineatus pyruvate dehydrogenase E1 component subunit alpha (NCBI Reference Sequence: XP_005341209.1).

\subsubsection{Multiplex Assays}

Luminex ${ }^{\circledR}$ assays were carried out in order to assess the relative levels of the three different regulatory phosphorylation sites between the euthermic and hibernating groups. This assay functions similarly to a flow cytometer and reads fluorescently labeled color-coded beads with a laser to identify which analyte is being measured, each bead bearing a capture antibody recognizing a specific phosphorylation site. Then the amount is quantified using the fluorescent intensity of a phycoerythrin-streptavidin chimera protein that is bound to a biotin labeled secondary antibody recognizing another epitope on the PDH surface. For these experiments, the Luminex ${ }^{\circledR}$ EMD Millipore PDHMAG13K kit was used to assess the PDH phosphorylation status during euthermia and hibernation for the ground squirrel tissues. 
Muscle and liver tissue homogenates were prepared for the multiplex assays by homogenizing with a Polytron homogenizer $\sim 50 \mathrm{mg}$ tissue samples from euthermic and hibernating animals in 1:5 w/v $1 \times$ lysis buffer (provided with the kit). The lysis buffer was supplemented with the addition of the following components: $1 \mathrm{mM} \mathrm{Na}_{3} \mathrm{VO}_{4}, 10 \mathrm{mM} \mathrm{NaF}, 10$ $\mathrm{mM} \beta$-glycerophosphate and $10 \mu \mathrm{l} / \mathrm{ml}$ protease inhibitor cocktail (BioShop \# PIC002.1). Following homogenization, the samples were sonicated quickly for $15 \mathrm{~s}$ to disrupt cell and mitochondrial membranes. Samples were then left on ice for $30 \mathrm{~min}$ with intermittent vortexing every $10 \mathrm{~min}$. Samples were subsequently centrifuged at $14,000 \times \mathrm{g}$ for $20 \mathrm{~min}$ and the supernatant was stored at $-72{ }^{\circ} \mathrm{C}$ until used.

The multiplex assay was performed according to the manufacturer's instructions as follows. The magnetic beads from the kit were sonicated for $10 \mathrm{~s}$, vortexed briefly and diluted to the working concentration. Beads were held overnight away from light under constant shaking at $4{ }^{\circ} \mathrm{C}$ in a 96 well microplate in either assay buffer (for a negative control) or in the previously described tissue lysates prepared to pre-determine optimal concentrations that were found to give the best results. Beads were separated from the lysate by using a magnetic separator block (\#40285) after which the contents of the wells were decanted and the wells were washed three times with assay buffer. The biotin-labeled antibodies were diluted to the proper concentration with assay buffer and this solution was added to the wells for a $1 \mathrm{~h}$ incubation periods at room temperature with constant shaking. Wells were then washed out as before and then the streptavidin-phycoerythrin conjugate protein was added for $30 \mathrm{~min}$ at room temperature. Amplification buffer was then added for 15 min with shaking at room temperature. The wells were again decanted and the beads were resuspended in assay buffer. The fluorescent intensity of each analyte was measured using a Luminex 100® instrument followed by data interpretation 
using xPonent software. The protein concentrations used were determined by using representative pooled samples of both tissues with protein set to varying amounts. An amount of protein found to give optimal results $(5 \mu \mathrm{g})$ along a linear portion of the standard curve was used in each well to assess the serine phosphorylation levels in the multiplex assays.

\subsubsection{PDH Activity Assays}

PDH activity assays were performed using an enzyme-linked immunosorbent assay (ELISA) based kit (abcam \#ab110671). This assay worked by binding PDH from a sample and then subsequently adding the substrate to the wells and the NADH produced by the reaction drove the reduction of a dye that in turn produced a yellow colour.

Samples were prepared for the assay using a $\sim 20$ piston strokes with a Dounce homogenizer on $\sim 0.2 \mathrm{~g}$ of tissue in $1 \mathrm{~mL}$ of $1 \times$ PBS buffer $\left(1.4 \mathrm{mM} \mathrm{KH}_{2} \mathrm{PO}_{4}, 8 \mathrm{mM} \mathrm{Na}_{2} \mathrm{HPO}_{4}\right.$, $140 \mathrm{mM} \mathrm{NaCl}, 2.7 \mathrm{mM} \mathrm{KCl}, 1 \mathrm{mM} \mathrm{Na} \mathrm{VO}_{4}, 10 \mathrm{mM} \mathrm{NaF}, 10 \mathrm{mM} \beta$-glycerophosphate, 10 $\mu \mathrm{L} / \mathrm{mL}$ protease inhibitor cocktail (BioShop, \#PIC002.1, $\mathrm{pH} 7.3$ )). To improve the yield of mitochondrial components from the lysate, 1 volume of detergent (supplied with the kit) was added for every 19 volumes of the lysate made and incubated on ice for $10 \mathrm{~min}$. These samples were then centrifuged at $1000 \times \mathrm{g}$ for $10 \mathrm{~min}$ at $4{ }^{\circ} \mathrm{C}$. The supernatant from this step was saved and the pellet was discarded. The supernatant was then assayed for protein concentration using the Bradford assay (Bradford, 1976) and diluted to the working concentration in $1 \times$ Buffer (supplied with the kit). The working concentrations were determined by performing the assay with varying concentrations of protein in both liver and muscle preparations. The working concentration for the liver was set to $5 \mathrm{mg} / \mathrm{mL}$ and for the muscle, it was set to $3.75 \mathrm{mg} / \mathrm{mL}$. 200 $\mu \mathrm{L}$ of each of the liver and muscle samples form the euthermic and hibernating animals were added to separate wells on the antibody bound microplate supplied with the kit including one 
well as a negative control that was loaded only with the Buffer. The microplate was then incubated at room temperature $\left(\sim 22^{\circ} \mathrm{C}\right)$ for $3 \mathrm{~h}$ with gentle rotation.

Following the incubation period, the wells were emptied and filled with $300 \mu \mathrm{L}$ of the $1 \times$ stabilizer solution included with the kit. The wells were emptied again and filled with another $300 \mu \mathrm{L}$ of the $1 \times$ stabilizer solution. $200 \mu \mathrm{L}$ of the assay solution (prepared as a mixture of the $20 \times$ Reagent Mix, $1 \times$ Buffer, $100 \times$ Coupler, and $100 \times$ Reagent dye in the proportions described by the manufacturer) was added to well. The absorbance of the dye was then monitored at $450 \mathrm{~nm}$ using a Biotek microplate reader set to intervals of $25 \mathrm{~s}$ with intermittent shaking of the plate at medium speed for $3 \mathrm{~s}$ for $1 \mathrm{~h}$. Following this, the data was analyzed using a microplate analysis program to determine the rate of absorbance increase in the wells (Brooks, 1994).

\subsubsection{Immunoblotting for Kinases and Phosphatases}

Western blotting techniques were used to assess the relative concentration of the pyruvate dehydrogenase kinases and phosphatase in the tissue homogenates. Muscle and liver tissues were weighed and homogenized in a 1:5 ratio w/v using a Polytron homogenizer in a buffer composed of the following components: $50 \mathrm{mM}$ Tris, $2 \mathrm{mM}$ EDTA, 2 mM EGTA, $20 \mathrm{mM} \beta-$ glycerophosphate, $10 \%$ glycerol v/v, pH 7.5). Samples were then centrifuged at 13,500 $\times \mathrm{g}$ for 30 min at $4{ }^{\circ} \mathrm{C}$ after which, the supernatant was kept while the pellet was discarded. Samples were then assayed for protein concentration using the Bradford method and were adjusted to a constant concentration between all samples $(10 \mu \mathrm{g} / \mu \mathrm{l})$. These samples were then mixed 1:1 with $1 \times$ SDS-loading buffer $(100 \mathrm{mM}$ Tris, $4 \% \mathrm{w} / \mathrm{v}$ SDS, $20 \% \mathrm{v} / \mathrm{v}$ glycerol, $0.2 \% \mathrm{w} / \mathrm{v}$ bromophenol blue, $\mathrm{pH}$ 6.8) with $10 \% \mathrm{v} / \mathrm{v} \beta$-mercaptoethanol and immersed in boiling water for $5 \mathrm{~min}$. These prepared samples were stored at $-72{ }^{\circ} \mathrm{C}$ until use. 
Western blotting was performed through electrophoretic separation of the protein samples in running buffer ( $25 \mathrm{mM}$ Tris, $250 \mathrm{mM}$ glycine, $0.1 \% \mathrm{w} / \mathrm{v}$ SDS) through $10 \%$ polyacrylamide gels at $180 \mathrm{~V}$ for 65 min using identical amounts of protein between samples in the range of 12.5-50 $\mu$ g. Proteins were then subsequently transferred to a PVDF membrane while immersed in transfer buffer (25 mM Tris, $192 \mathrm{mM}$ glycine, $20 \% \mathrm{v} / \mathrm{v}$ methanol, $\mathrm{pH} 8.8)$ at $210 \mathrm{~mA}$ constant current for $90 \mathrm{~min}$. Blots were blocked using skim milk powder at concentrations ranging from 1 to $4 \% \mathrm{w} / \mathrm{v}$ in TBST (20 mM Tris base, $140 \mathrm{mM} \mathrm{NaCl}, 0.05 \% \mathrm{v} / \mathrm{v}$ Tween-20, $\mathrm{pH} 7.6$ ) for $30 \mathrm{~min}$. Blots were then incubated overnight with an antibody recognizing one of the PDKs or PDP1 diluted 1:1000 from the original concentration in TBST. The primary antibody was then removed and the blots were washed $3 \times 5 \mathrm{~min}$ in TBST to remove excess primary antibody. The blots were then incubated for $30 \mathrm{~min}$ in anti-rabbit IgG-HRP conjugated antibody diluted 1:8000 in TBST. The blots were again washed $3 \times 5 \mathrm{~min}$ in TBST before being visualized in a ChemiGenius Bioimaging System (Syngene, Frederick, MD) instrument using an enhanced luminescence (ECL) protocol. After a suitable image was developed, the blots were stained with Coomassie brilliant blue stain until sufficient contrast was developed. These stained blots were imaged using the same instrument as before with internal lighting turned on. The signal from the ECL was normalized against a group of stably expressed protein bands in the Coomassie image of the picture (Bradford, 1976; Eaton et al., 2013). Images were analyzed using GeneTools software (v3.00.02). The antibodies used in the experiments were as follows:

\section{PDK1 (GeneTex, GTX105999)}

2. PDK2 (GeneTex, GTX103251) 


\section{PDK3 (GeneTex, GTX104286)}

\section{PDK4 (GeneTex, GTX105667)}

\section{PDP1 (GeneTex, GTX109533)}

\subsubsection{Statistical Analysis and Software}

Data was analyzed and graphs were generated using RBioplot software (Zhang and Storey, 2016). This software determines statistical differences through a Student's t-test when $\mathrm{p}<0.05$. Data represents a mean of 4-5 independent replicates from both euthermic and hibernating Richardson's ground squirrels. Data are presented relative to the euthermic value for each parameter and are adjusted by dividing all values (both euthermic and hibernating) by the mean of the euthermic parameter. Error represents the standard error of the mean of the adjusted values.

\subsection{Results}

\subsubsection{Sequence Similarity}

The amino acid sequence alignment of the PDH E1 $\alpha$ subunits demonstrates a very high degree of similarity (99.23\% identical) between the human and ground squirrel sequences, strongly suggesting that the antibodies used in the Luminex assay and the ELISA activity kit would recognize the PDH measured in the subsequent experiments. Additionally, all of the serine sites known to have important regulatory functions through phosphorylation were shown to be conserved in the ground squirrel sequence (Fig. 2.1).

\subsubsection{PDH Phosphorylation}

Multiplex assays demonstrated no difference between the control euthermic skeletal muscle tissue samples and the hibernating samples. This was true of all three phosphorylation 
states (S232, S293, and S300) as well as the total PDH levels (Fig. 2.2.). This was in contrast to the liver samples that showed a significant increase in the levels of S300 phosphorylation (2.17fold increase). Levels of PDH in the liver remained unchanged in response to hibernation (Fig. 2.3.).

\subsubsection{PDH Activity}

Levels of PDH activity in the skeletal muscle remained unchanged relative to the control euthermic levels. This was in contrast to the liver where a large decrease $(0.149$-fold change) occurred during hibernation when compared to the euthermic.

\subsubsection{PDH Kinase and Phosphatase Expression}

In the liver of the ground squirrel, no changes were seen associated with hibernation in the expression of any of the 4 kinases that are known to affect the phosphorylation state of PDH (Fig. 2.4.). Interestingly, in the skeletal muscle there were significant increases in PDK1 and PDK2 expression and lowered PDK4 expression (Fig. 2.5.). Quantification of the PDH phosphatase catalytic subunit demonstrated no significant differences between the euthermic controls and the hibernating animals in either the liver or the skeletal muscle tissue (Fig. 2.5.).

\subsection{Discussion}

Given the crucial role of PDH in controlling the entry of acetyl CoA into the mitochondria from the glycolytic end-product pyruvate, it is unsurprising that regulation of this enzyme is a well-conserved feature across all taxa. Performing a sequence alignment of the 13lined ground squirrel, the closest relative of the Richardson's ground squirrel for which a sequence exists, and the human sequence E1 $\alpha$ demonstrate that only 3 out of all 390 of the amino acids vary between the two species. The extremely low degree of variation is likely a 
consequence of the need to ensure the structural stability of the complex since even small changes could disrupt the integration of the protein into the complex (Kisters-Woike et al., 2000). Additionally, this high degree of similarity suggests that the interactions between the PDKs and their peptide substrates are likely constant and probably function in the same manner between humans and ground squirrels. This makes PDH an ideal target for cross-species comparison since antibody-based tools designed for use in one animal have a very high likelihood of functioning properly in another species. Indeed studies from our research group have previously used this same multiplex assay kit in the context of numerous other vertebrate species ranging from the wood frog (Rana sylvatica) to a small marsupial species (Dromiciops gliroides) (Al-attar et al., 2019; Luu et al., 2018). In the current study, magnetic fluorescently labeled bead-based multiplex assays were used to rapidly characterize the phosphorylation states of the three critical regulatory serine residues and then an ELISA-based assay was used to quantify the activity in the liver and muscle.

In the muscle tissue, no changes were detected either in the degree of PDH phosphorylation or in PDC activity associated with the animal entering into hibernation. The lack of change detected in activity in the muscle was consistent with what was expected given the absence of measurable changes in the degree of PDH phosphorylation and suggests that both techniques were reliable in assessing their respective parameters. Increased phosphorylation on the 3 serine sites, including S300 of PDH which was elevated during torpor in the liver, is known to be associated with a decrease in activity in the recombinant human sequence through sitedirected mutagenesis experiments (Korotchkina and Patel, 1995). However, the lack of change in phosphorylation during hibernation does seem somewhat odd considering the great reduction in metabolic rate that occurs during hibernation. It is possible that upstream changes in glycolytic 
function already limit the quantity of substrates available for conversion into acetyl-CoA thereby limiting the need for control at PDH. This is supported by previous studies which have suggested in this animal that hibernation is associated with a reduction in the activity of key glycolytic enzymes in the muscle tissue during hibernation (Abnous and Storey, 2008; Bell and Storey, 2018). Additionally, the reduction in temperature during winter hibernation may help to reduce the activity of the complex without the need of more active restriction in the activity of the complex through post-translational modification. One study showed that PDH activity increases linearly with assay temperatures ranging from $8{ }^{\circ} \mathrm{C}$ to $22{ }^{\circ} \mathrm{C}$ in a fish species with a $\sim 2$-fold increase in activity at $22{ }^{\circ} \mathrm{C}$ compared to $8{ }^{\circ} \mathrm{C}$ (Blier and Guderley, 1993). This suggests that the true difference between euthermic and hibernating liver PDH activity is even higher than the data here would suggest and also that a passive reduction of PDH activity in the muscle would occur despite the lack of changes in phosphorylation. Mitochondria in endothermic animals are very often $\sim 10{ }^{\circ} \mathrm{C}$ higher than the rest of the cell, meaning that in euthermic mammals the mitochondrial temperature is likely around $50^{\circ} \mathrm{C}$ (Chrétien et al., 2018). It is currently unknown how mitochondrial temperature differs from body temperature during hibernation, but given the reduction in central metabolic rate that accompanies hibernation it seems likely that the relatively elevated temperatures that normally characterize the mitochondria might be lessened, thereby further contributing to slowing PDH activity.

The inhibitory phosphorylation events of PDH are mediated by a series of PDH kinases (PDKs) that are differentially distributed throughout the tissues (with PDK1 and 2 having the widest tissue distribution) of animals and are generally thought to affect different sites (Klyuyeva et al., 2019; Korotchkina and Patel, 2001). Using immunoblotting to assess the cellular concentration of the PDKs revealed some surprising results in both the liver and muscle. While 
the liver demonstrated a substantial inhibition in PDH activity during hibernation relative to the euthermic controls and this was correlated with a significant increase in S300 phosphorylation, no changes in the relative level of kinases or of the catalytic subunit of the phosphatase were demonstrated in the liver. Previous studies have suggested that PDK3 and to a lesser degree PDK4 exhibit the greatest capacity to phosphorylate at the S300 residue and the lack of change of these in particular in the liver is somewhat odd (Korotchkina and Patel, 2001). On the other hand, in the muscle, significant differences were observed neither in the activity of PDH nor in the phosphorylation level but a significant increase in PDKs 1 and 2 and a slight decrease in PDK4 was observed. The increase of PDK3 expression may be partly offset by the decrease in PDK4 since PDK3 only has a slightly higher affinity for the S300 site in PDH (Korotchkina and Patel, 2001). The lack of change in the S293 site could also be explained in a similar manner since PDK2 has the greatest capacity to phosphorylate this residue and is followed by PDK4. Constant S232 phosphorylation is more difficult to explain, as PDK1 is the only known of the kinases that phosphorylates at this site (Korotchkina and Patel, 2001). This seeming contradiction suggests that the concentration of PDKs do not necessarily directly predict the phosphorylation level of PDH in the cell. To further investigate, the concentration of PDP1 (the catalytic subunit of the phosphatase responsible for dephosphorylation of the PDH) were assessed in the two tissues. No changes were observed in this protein suggesting that the levels of this protein are not responsible for the disjunction between the observed PDK levels and the phosphorylation levels. While no changes were observed in the overall concentration of this phosphatase in the samples, it is possible that phosphatase activity may be differentially regulated through other means. Notably, the activity of the phosphatase is controlled through the mitochondrial levels of calcium through the level of mitochondrial calcium uniporter (MCU) or 
other factors affecting its function or mitochondrial calcium concentration in general (Lander et al., 2018). To date no studies have looked at $\mathrm{Ca}^{+}{ }^{+}$homeostasis in the liver of a hibernator, however in another species of ground squirrel, the Daurian ground squirrel (Spermophilus dauricus), increased levels of MCU expression are observed in the skeletal muscle during late torpor suggesting that $\mathrm{Ca}^{+}$levels may increase during this period (Zhang et al., 2019). This may help to explain the lack of increase in phosphorylation particularly on the S232 site that could not be explained by the decreased expression of PDK4 in the muscle during hibernation. Several other possibilities may help to resolve this paradox, such as the possibility that post-translational modification may contribute to the regulation of PDP1 activity as has been observed through the inhibition of phosphatase activity through phosphorylation of Tyr-94 (Shan et al., 2014). To date little is known about the regulation of PDKs through phosphorylation or other PTMs although these enzymes are known to be controlled through feedback inhibition with high levels of acetylCoA and NADH causing stimulation of PDK activity thereby turning off PDH (Pettit et al., 1975). The results shown demonstrate that PDK phosphorylation level and activity does not necessarily correlate with the levels of PDH kinases or phosphatases, suggesting that other mechanisms are involved in the regulation of PDK and PDP activity.

Owing to its central importance in determining metabolic rate, regulation of the PDH has been the subject of numerous studies with regards to its function in metabolically depressed states (Hadj-Moussa et al., 2018). A similar, albeit much more dramatic, effect was observed in hibernating 13-lined ground squirrels (Ictidomys tridecemlineatus) where in the liver (but not in muscle) the levels of S300 phosphorylation were found to be increased during hibernation at over 30-fold during late torpor (Wijenayake et al., 2017). This current study adds to the former by clearly demonstrating that ground squirrel PDH in the more highly phosphorylated S300 state 
has greatly decreased activity. In the 13-lined ground squirrel the level of PDK1 tended to correlate well with the S300 phosphorylation pattern, although this pattern was not seen in this study perhaps due to the magnitude of the phosphorylation effect being lower in the current study. Increases in levels of S300 phosphorylation in the liver during torpor in a small marsupial species (Dromiciops gliroides) were also observed concurrently with increases in S293. This suggests that although some similarities exist between different animals, the specific phosphorylation sites that are enhanced during torpor tend to vary based on species (Wijenayake et al., 2018). Because the mechanisms of PDH control are well conserved across all vertebrates, similar methodology has demonstrated that a species of frog also has elevated S300 phosphorylation during whole-body winter freezing and this trend is reversed upon thawing in the liver (Al-attar et al., 2019). Interestingly the liver does not necessarily follow a strong pattern of serine phosphorylation based suppression in a primate model of torpor (Microcebus murinus), but rather showed strong signs of suppression in the skeletal muscle (Wijenayake et al., 2018). However, this study did not measure the actual PDH activity in the tissues so it is possible that other less canonical mechanisms of PDH control such as phosphorylation of Y301 could induce PDH suppression in the liver and other tissues (Fan et al., 2014). Taken together with the studies performed here, inhibition of PDC activity through inhibitory phosphorylation seems to be a common mechanism for animals to employ in facilitating metabolic depression.

\subsection{Conclusions}

The current study characterized the relative phosphorylation levels of the three critical serine residues responsible for regulating the activity of PDH in the skeletal muscle and liver of hibernating and euthermic Richardson's ground squirrels. This work also demonstrated the relative activity of PDH during the torpid and active states. The results demonstrate a clear 
increase in the level of S300 phosphorylation in the liver, but not muscle, and that levels of this PTM are correlated with inhibition of the activity of this enzyme. Interestingly, investigating the expression patterns of the four PDKs did not reveal any significant differences in the liver, suggesting that other mechanisms may be involved in regulating the level of phosphorylation in this tissue during hibernation beyond mere changes in protein expression. The results support a growing body of evidence demonstrating that the tissue specific regulation of PDH is a critical element of mediating metabolic rate depression in adaptations to adverse environments.

\section{Author statement}

S.R. Green: Formal analysis; Investigation; Methodology; Visualization; Writing - original draft.

K.B. Storey: Conceptualization; Funding acquisition; Project administration; Writing - review \& editing; Supervision.

\section{Declaration of competing interest}

The authors declare no competing interests in the publication or experiments performed in this manuscript.

\section{Acknowledgements}

Research was funded by a Natural Sciences and Engineering Research Council of Canada (NSERC) Discovery grant (\#6793). K.B.S. holds the Canada Research Chair in Molecular Physiology. S.R.G. held a NSERC Alexander Graham Bell Canada Graduate Scholarship at the doctoral level (CGS-D) and an Ontario Graduate Scholarship while performing the experiments. The authors would like to extend thanks to J.M. Storey for review of the manuscript and for providing feedback. 

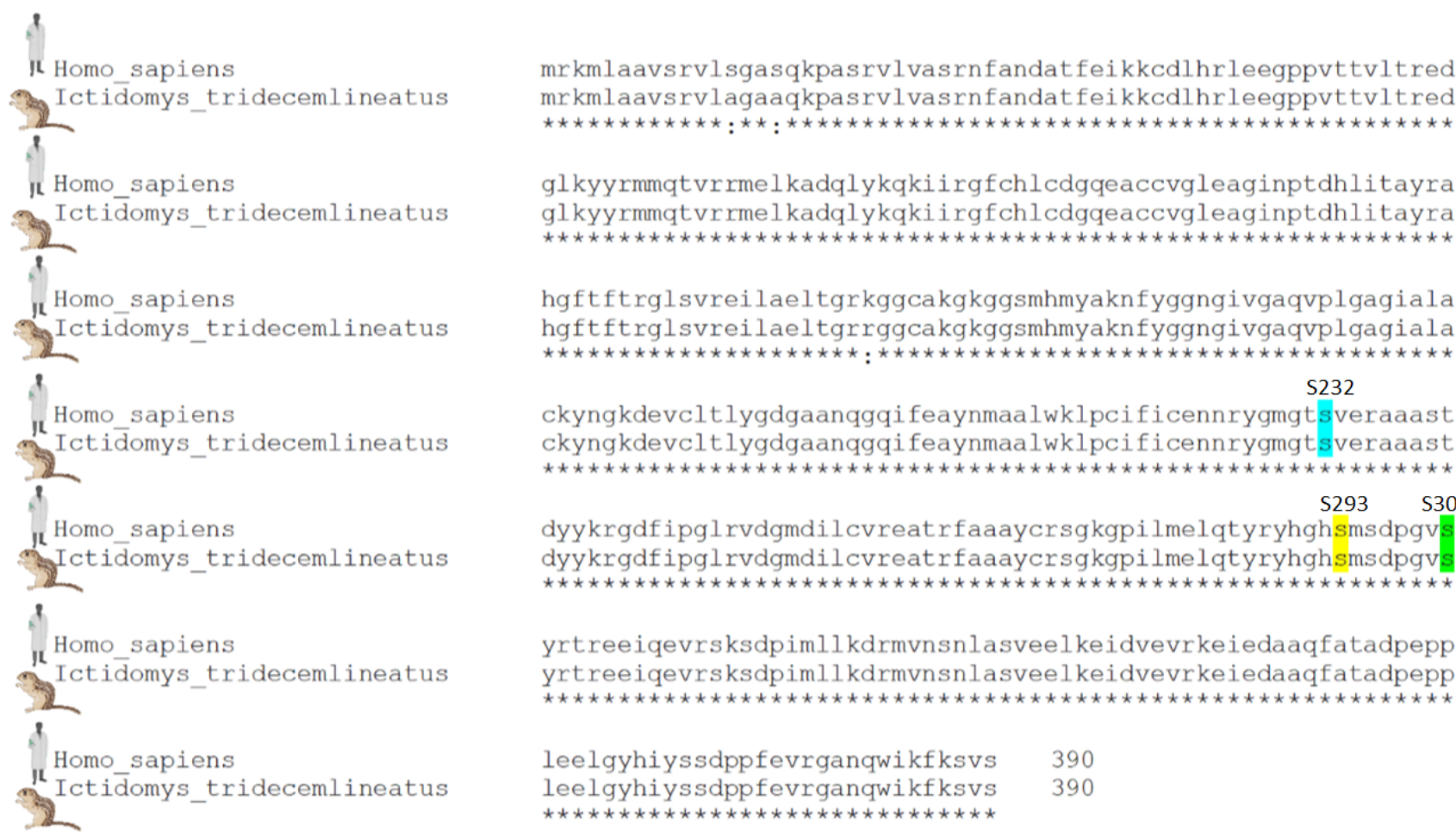

Fig. 2.1. Sequence alignment using Clustal Omega Multiple Sequence Alignment Tool of the closest related species to the Richardson's ground squirrel (Ictidomys tridecemlineatus) to the human PDH E1 $\alpha$ subunit. The serine phosphorylation sites known to have a critical role in regulating the activity of the complex are highlighted and labeled. '*' below a certain residue means that both sequences are identical at that position, whereas ':' indicates that the amino acid has been substituted for one with similar properties. 

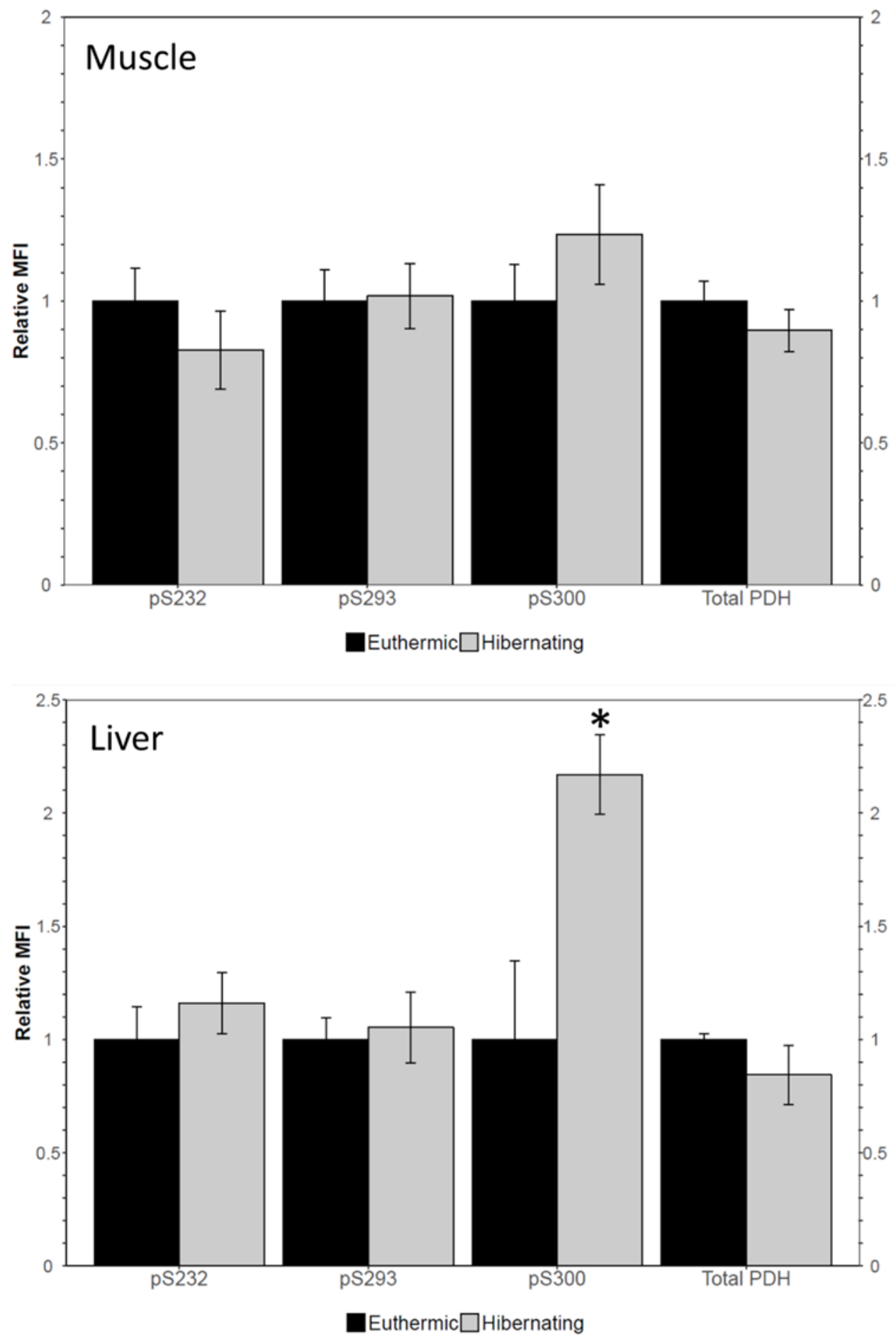

Fig. 2.2. The relative degree of phosphorylation on specific regulatory sites of the pyruvate dehydrogenase E1 $\alpha$ subunit and total PDH levels from control euthermic and hibernating muscle (top) and liver (bottom) tissue of the Richardson's ground squirrel (Urocitellus richardsonii). Data are presented with control euthermic mean set to 1 . Data represent the mean \pm standard error of the mean, $n=5$ independent replicates. ' $*$ ' indicates that the hibernating parameter is significantly different from the euthermic ( $\mathrm{p}<0.05$, Student's $t$-test). 


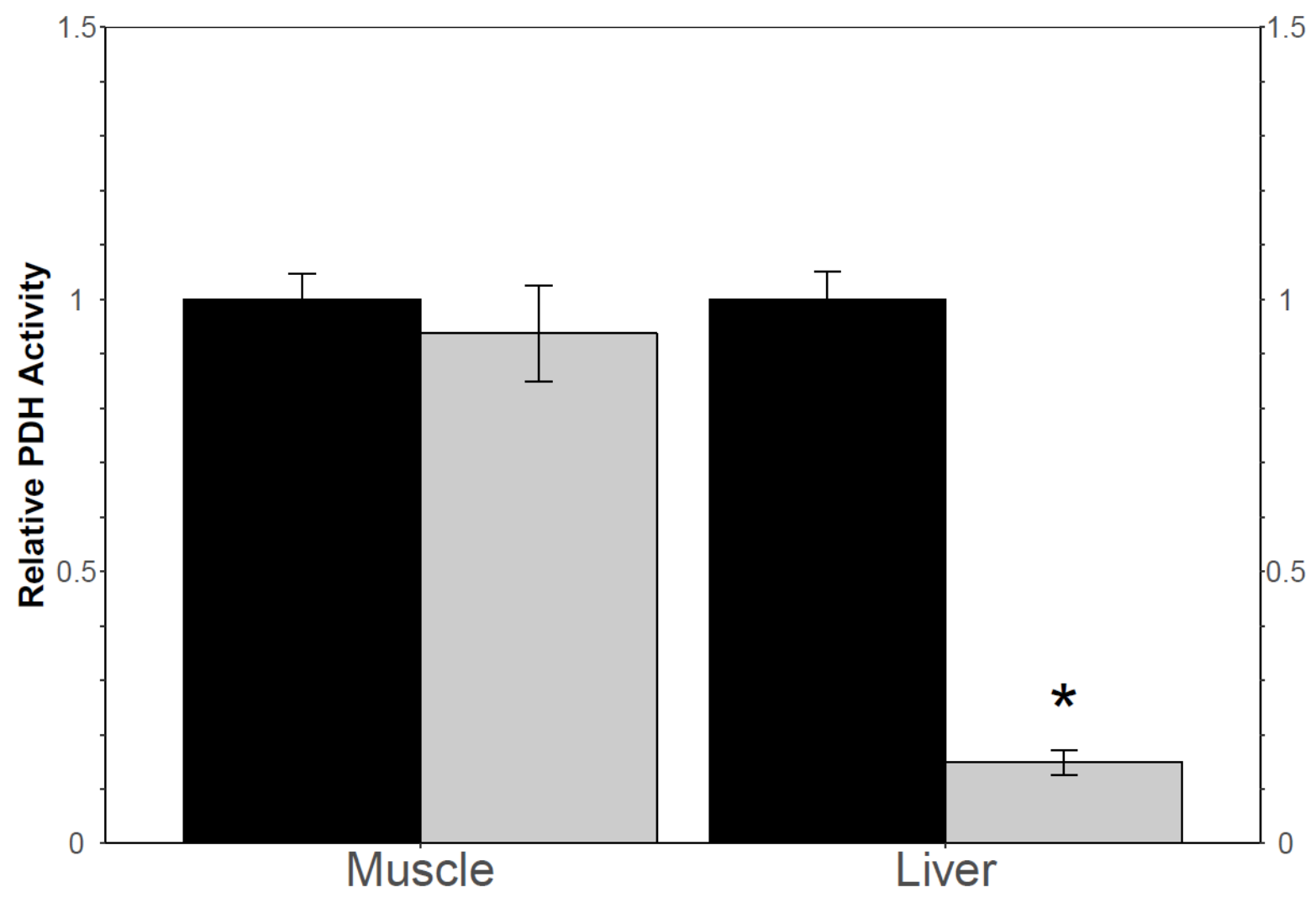

Euthermic $\square$ Hibernating

Fig. 2.3. The relative level of pyruvate dehydrogenase activity in tissue homogenates prepared from euthermic and hibernating Richardson's ground squirrels. Data are presented as means \pm standard error of the mean from $n=4$ independent replicates. The euthermic mean value was set to 1 and the corresponding hibernating parameter is presented relative to the euthermic. '*' indicates that the hibernating value is significantly different from the euthermic $(p<0.05$, Student's $t$-test). 

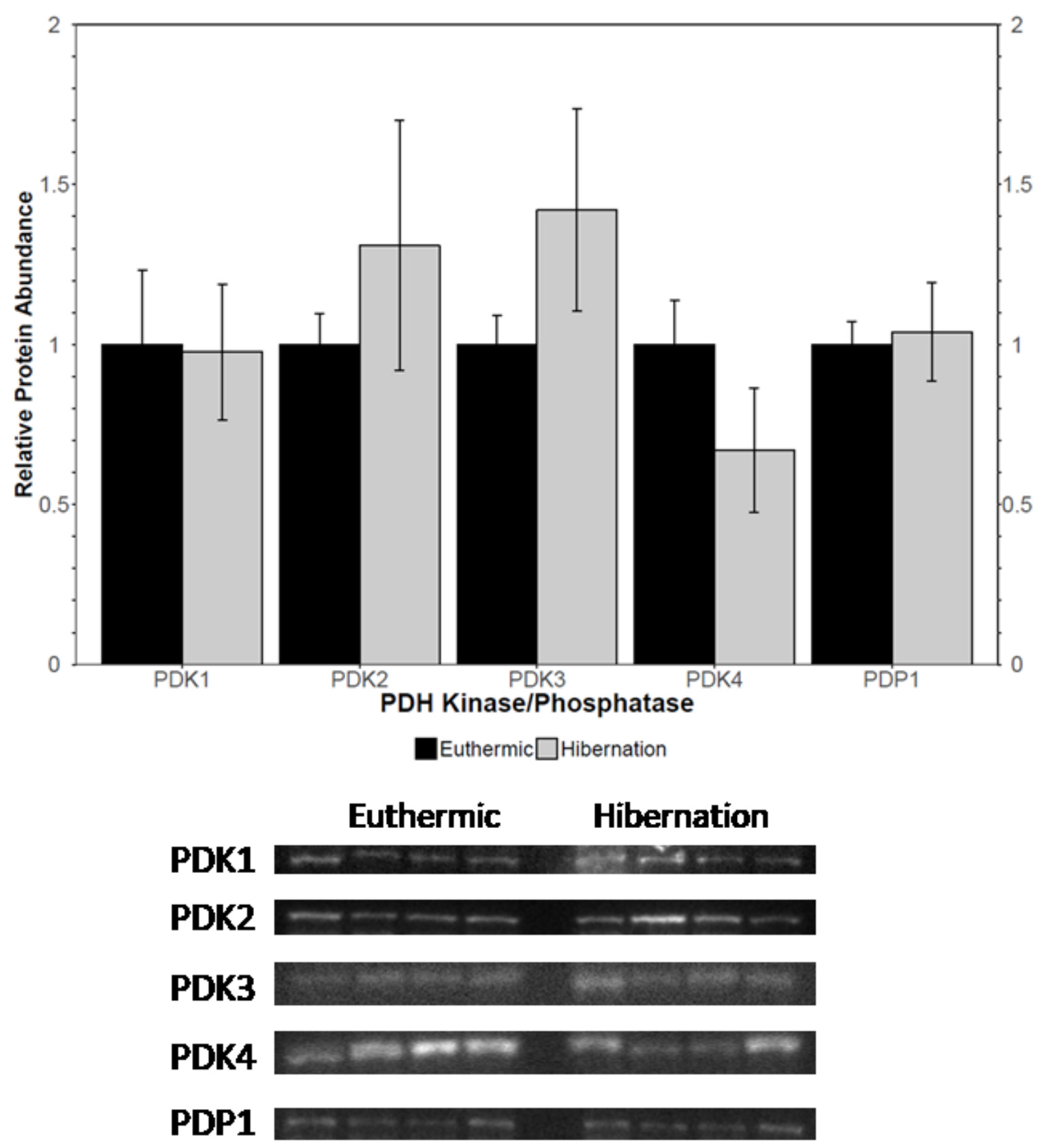

Fig. 2.4. Quantification of the kinases and the catalytic subunit of pyruvate dehydrogenase phosphatase in the liver of the Richardson's ground squirrel from euthermic and hibernating animals determined from immunoblotting experiments. Data represents a mean of $n=4$ independent replicates with error represented as standard error of the mean. Data is presented relative to the euthermic control set to a value of 1 . No significant differences were observed in the data when a Student's $t$-test was applied at the $\mathrm{p}<0.05$ level. 

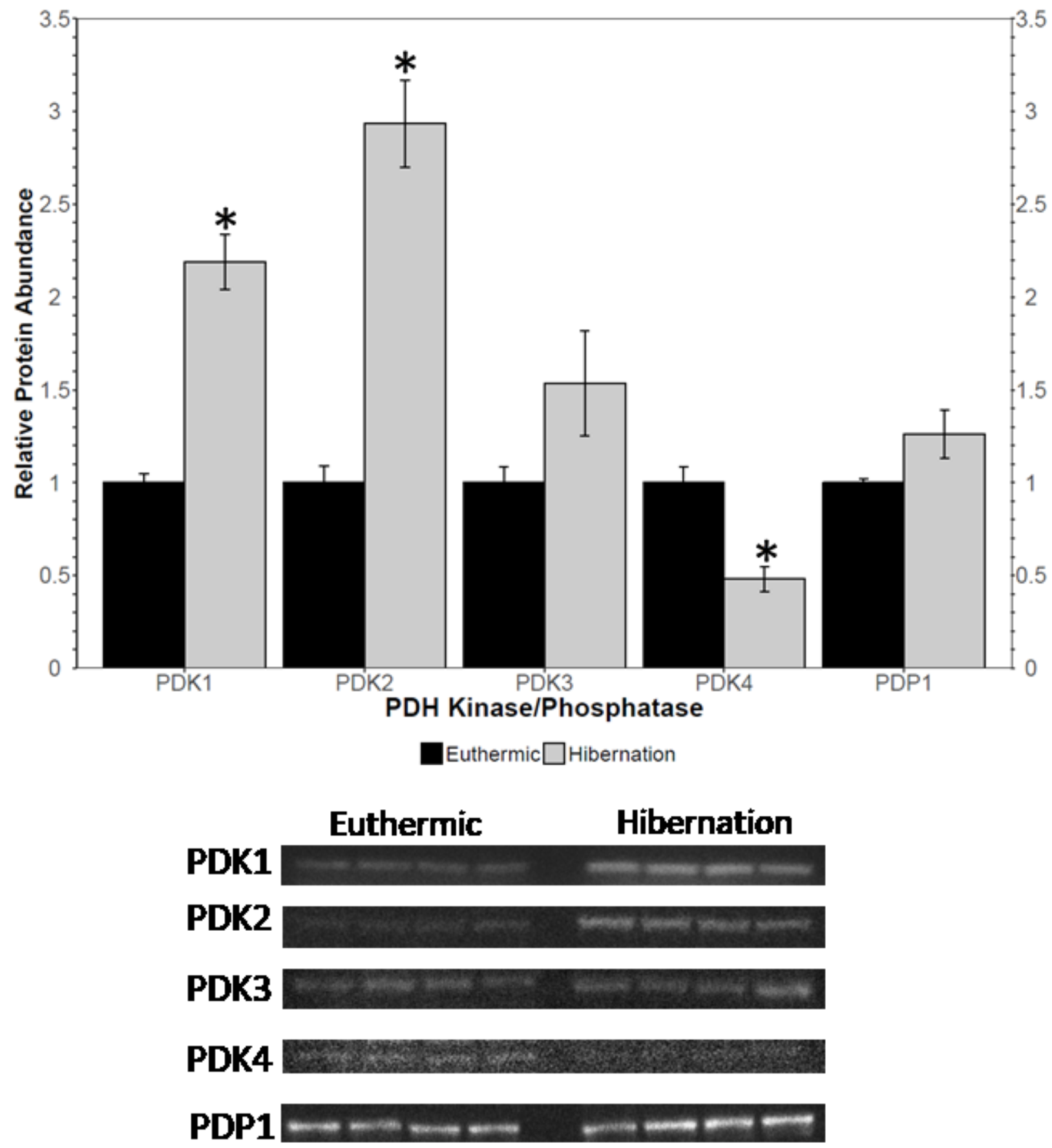

Fig. 2.5. Quantification of the kinases and the catalytic subunit of pyruvate dehydrogenase phosphatase in the muscle of the Richardson's ground squirrel from euthermic and hibernating animals determined from immunoblotting experiments. Data represents a mean of $n=4$ independent replicates with error represented as standard error of the mean. Data is presented relative to the euthermic control set to a value of 1 . Significant differences are marked with '*' when $\mathrm{p}<0.05$ as determined by a Student's $t$-test. 


\section{Chapter 2 References}

Abnous, K., \& Storey, K. B. (2008). Skeletal muscle hexokinase: Regulation in mammalian hibernation. Molecular and Cellular Biochemistry, 319(1-2), 41-50. https://doi.org/10.1007/s11010-008-9875-5

Al-attar, R., Wijenayake, S., \& Storey, K. B. (2019). Metabolic reorganization in winter: Regulation of pyruvate dehydrogenase (PDH) during long-term freezing and anoxia. Cryobiology, 86, 10-18. https://doi.org/10.1016/j.cryobiol.2019.01.006

Bell, R. A. V., \& Storey, K. B. (2018). Purification and characterization of skeletal muscle pyruvate kinase from the hibernating ground squirrel, Urocitellus richardsonii: potential regulation by posttranslational modification during torpor. Molecular and Cellular Biochemistry, 442(1-2), 47-58. https://doi.org/10.1007/s11010-017-3192-9

Blier, P. U., \& Guderley, H. E. (1993). Effects of $\mathrm{pH}$ and temperature on the kinetics of pyruvate oxidation by muscle mitochondria from rainbow trout (Oncorhynchus mykiss). Physiological Zoology, 66(4), 474-489. https://doi.org/10.1086/physzool.66.4.30163804

Bradford, M. M. (1976). A rapid and sensitive method for the quantitation of microgram quantities of protein utilizing the principle of protein-dye binding. Analytical Biochemistry, 72(1-2), 248-254. https://doi.org/10.1016/0003-2697(76)90527-3

Brooks, S. P. (1994). A program for analyzing enzyme rate data obtained from a microplate reader. Biotechniques, 17(6), 1154-1161. Retrieved from http://www.ncbi.nlm.nih.gov/entrez/query.fcgi? $\mathrm{cmd}=$ Retrieve\&db=PubMed\&dopt=Citation \&list_uids $=7873186$

Chrétien, D., Bénit, P., Ha, H. H., Keipert, S., El-Khoury, R., Chang, Y. T., ... Rak, M. (2018). Mitochondria are physiologically maintained at close to $50{ }^{\circ} \mathrm{C}$. PLoS Biology, 16(1). https://doi.org/10.1371/journal.pbio.2003992

Eaton, S. L., Roche, S. L., Llavero Hurtado, M., Oldknow, K. J., Farquharson, C., Gillingwater, T. H., \& Wishart, T. M. (2013). Total protein analysis as a reliable loading control for quantitative fluorescent western blotting. PLoS ONE, 8(8), e72457. https://doi.org/10.1371/journal.pone.0072457

Fan, J., Kang, H. B., Shan, C., Elf, S., Lin, R., Xie, J., ... Chen, J. (2014). Tyr-301 phosphorylation inhibits pyruvate dehydrogenase by blocking substrate binding and promotes the warburg effect. Journal of Biological Chemistry, 289(38), 26533-26541. https://doi.org/10.1074/jbc.M114.593970

Geiser, F. (1988). Reduction of metabolism during hibernation and daily torpor in mammals and birds: temperature effect or physiological inhibition? Journal of Comparative Physiology B, 158(1), 25-37. https://doi.org/10.1007/BF00692726

Gey, U., Czupalla, C., Hoflack, B., Rödel, G., \& Krause-Buchholz, U. (2008). Yeast pyruvate dehydrogenase complex is regulated by a concerted activity of two kinases and two 
phosphatases. Journal of Biological Chemistry, 283(15), 9759-9767.

https://doi.org/10.1074/jbc.M708779200

Green, S. R., \& Storey, K. B. (2020). Regulation of the $\alpha$-ketoglutarate dehydrogenase complex during hibernation in a small mammal, the Richardson's ground squirrel (Urocitellus richardsonii). Biochimica et Biophysica Acta - Proteins and Proteomics, 1868(9). https://doi.org/10.1016/j.bbapap.2020.140448

Hadj-Moussa, H., Green, S. R., \& Storey, K. B. (2018). The living dead: mitochondria and metabolic arrest. IUBMB Life, 70(2), 1260-1266. https://doi.org/10.1002/iub.1910

Holness, M. J., \& Sugden, M. C. (2003). Regulation of pyruvate dehydrogenase complex activity by reversible phosphorylation. In Biochemical Society Transactions (Vol. 31, pp. 11431151). https://doi.org/10.1042/bst0311143

Huang, B., Wu, P., Bowker-Kinley, M. M., \& Harris, R. A. (2002). Regulation of pyruvate dehydrogenase kinase expression by peroxisome proliferator-activated receptor- $\alpha$ ligands, glucocorticoids, and insulin. Diabetes, 51(2), 276-283. https://doi.org/10.2337/diabetes.51.2.276

Kisters-Woike, B., Vangierdegom, C., \& Müller-Hill, B. (2000). On the conservation of protein sequences in evolution. Trends in Biochemical Sciences. https://doi.org/10.1016/S09680004(00)01631-5

Klyuyeva, A., Tuganova, A., Kedishvili, N., \& Popov, K. M. (2019). Tissue-specific kinase expression and activity regulate flux through the pyruvate dehydrogenase complex. Journal of Biological Chemistry, 294(3), 838-851. https://doi.org/10.1074/jbc.RA118.006433

Korotchkina, L. G., \& Patel, M. S. (1995). Mutagenesis studies of the phosphorylation sites of recombinant human pyruvate dehydrogenase. Site-specific regulation. Journal of Biological Chemistry, 270(24), 14297-14304. https://doi.org/10.1074/jbc.270.24.14297

Korotchkina, L. G., \& Patel, M. S. (2001). Site specificity of four pyruvate dehydrogenase kinase isoenzymes toward the three phosphorylation sites of human pyruvate dehydrogenase.

Journal of Biological Chemistry, 276(40), 37223-9.

https://doi.org/10.1074/jbc.M103069200

Lander, N., Chiurillo, M. A., Bertolini, M. S., Storey, M., Vercesi, A. E., \& Docampo, R. (2018). Calcium-sensitive pyruvate dehydrogenase phosphatase is required for energy metabolism, growth, differentiation, and infectivity of Trypanosoma cruzi. Journal of Biological Chemistry, 293(45), 17402-17417. https://doi.org/10.1074/jbc.RA118.004498

Li, X., Wu, F., Qi, F., \& Beard, D. A. (2011). A database of thermodynamic properties of the reactions of glycolysis, the tricarboxylic acid cycle, and the pentose phosphate pathway. Database, 2011. https://doi.org/10.1093/database/bar005

Lissens, W., De Meirleir, L., Seneca, S., Liebaers, I., Brown, G. K., Brown, R. M., ... Seyda, A. (2000). Mutations in the X-linked pyruvate dehydrogenase (E1) $\alpha$ subunit gene (PDHA1) in 
patients with a pyruvate dehydrogenase complex deficiency. Human Mutation. https://doi.org/10.1002/(SICI)1098-1004(200003)15:3<209::AID-HUMU1>3.0.CO;2-K

Luu, B. E., Wijenayake, S., Zhang, J., Tessier, S. N., Quintero-Galvis, J. F., Gaitán-Espitia, J. D., ... Storey, K. B. (2018). Strategies of biochemical adaptation for hibernation in a South American marsupial, Dromiciops gliroides : 2. Control of the Akt pathway and protein translation machinery. Comparative Biochemistry and Physiology Part B: Biochemistry and Molecular Biology, 224, 19-25. https://doi.org/10.1016/j.cbpb.2017.12.006

Mathers, K. E., \& Staples, J. F. (2019). Differential posttranslational modification of mitochondrial enzymes corresponds with metabolic suppression during hibernation. American Journal of Physiology-Regulatory, Integrative and Comparative Physiology, 317(2), R262-R269. https://doi.org/10.1152/ajpregu.00052.2019

Patel, M. S., Nemeria, N. S., Furey, W., \& Jordan, F. (2014). The pyruvate dehydrogenase complexes: Structure-based function and regulation. Journal of Biological Chemistry. https://doi.org/10.1074/jbc.R114.563148

Pettit, F. H., Pelley, J. W., \& Reed, L. J. (1975). Regulation of pyruvate dehydrogenase kinase and phosphatase by acetyl-CoA/CoA and NADH/NAD ratios. Biochemical and Biophysical Research Communications, 65(2), 575-582. https://doi.org/10.1016/S0006-291X(75)801859

Shan, C., Kang, H. B., Elf, S., Xie, J., Gu, T. L., Aguiar, M., ... Fan, J. (2014). Tyr-94 phosphorylation inhibits pyruvate dehydrogenase phosphatase 1 and promotes tumor growth. Journal of Biological Chemistry, 289(31), 21413-21422. https://doi.org/10.1074/jbc.M114.581124

Sievers, F., Wilm, A., Dineen, D., Gibson, T. J., Karplus, K., Li, W., ... Higgins, D. G. (2011). Fast, scalable generation of high-quality protein multiple sequence alignments using Clustal Omega. Molecular Systems Biology, 7, 539. https://doi.org/10.1038/msb.2011.75

Smolinski, M. B., Green, S. R., \& Storey, K. B. (2020). Characterizing the regulation of pyruvate kinase in response to hibernation in ground squirrel liver (Urocitellus richardsonii). Comparative Biochemistry and Physiology Part - B: Biochemistry and Molecular Biology, 248-249. https://doi.org/10.1016/j.cbpb.2020.110466

Van Breukelen, F., \& Martin, S. L. (2015). The hibernation continuum: Physiological and molecular aspects of metabolic plasticity in mammals. Physiology, 30(4), 273-281. https://doi.org/10.1002/aja.1000900202

Vassylyev, D. G., \& Symersky, J. (2007). Crystal Structure of Pyruvate Dehydrogenase Phosphatase 1 and its Functional Implications. Journal of Molecular Biology, 370(3), 417426. https://doi.org/10.1016/j.jmb.2007.05.002 
Wang, L. (1973). Radiotelemetric study of hibernation under natural and laboratory conditions. American Journal of Physiology-Legacy Content, 224(3), 673-677. https://doi.org/10.1152/ajplegacy.1973.224.3.673

Wang, L. C. H. (1979). Time patterns and metabolic rates of natural torpor in the Richardson's ground squirrel. Canadian Journal of Zoology, 57(2), 149-155. https://doi.org/10.1139/z79012

Wang, L. C. H., \& Lee, T. F. (1996). Torpor and hibernation in mammals: Metabolic, physiological, and biochemical adaptations. Handbook of Physiology - Environmental Physiology, 507-532. https://doi.org/10.1002/cphy.cp040122

Weraduwage, S. M., Micallef, M. C., Marillia, E. F., Taylor, D. C., Grodzinski, B., \& Micallef, B. J. (2016). Increased mtPDH activity through antisense inhibition of mitochondrial pyruvate dehydrogenase kinase enhances inflorescence initiation, and inflorescence growth and harvest index at elevated $\mathrm{CO} 2$ in Arabidopsis thaliana. Frontiers in Plant Science, 7(FEB2016). https://doi.org/10.3389/fpls.2016.00095

Wijenayake, S., Luu, B. E., Zhang, J., Tessier, S. N., Quintero-Galvis, J. F., Gaitán-Espitia, J. D., ... Storey, K. B. (2018). Strategies of biochemical adaptation for hibernation in a South American marsupial, Dromiciops gliroides: 4. Regulation of pyruvate dehydrogenase complex and metabolic fuel selection. Comparative Biochemistry and Physiology Part - B: Biochemistry and Molecular Biology, 224, 32-37. https://doi.org/10.1016/j.cbpb.2017.12.008

Wijenayake, S., Tessier, S. N., \& Storey, K. B. (2017). Regulation of pyruvate dehydrogenase (PDH) in the hibernating ground squirrel, (Ictidomys tridecemlineatus). Journal of Thermal Biology, 69, 199-205. https://doi.org/10.1016/j.jtherbio.2017.07.010

Zhang, J., Li, X., Ismail, F., Xu, S., Wang, Z., Peng, X., ... Gao, Y. (2019). Priority strategy of intracellular $\mathrm{Ca} 2+$ homeostasis in skeletal muscle fibers during the multiple stresses of hibernation. Cells. https://doi.org/10.3390/cells9010042

Zhang, J., \& Storey, K. B. (2016). RBioplot: an easy-to-use R pipeline for automated statistical analysis and data visualization in molecular biology and biochemistry. PeerJ, 4, e2436. https://doi.org/10.7717/peerj.2436

Zhang, S., Hulver, M. W., McMillan, R. P., Cline, M. A., \& Gilbert, E. R. (2014). The pivotal role of pyruvate dehydrogenase kinases in metabolic flexibility. Nutrition and Metabolism. https://doi.org/10.1186/1743-7075-11-10

Zhuang, Y., Ly, R. C., Frazier, C. V., Yu, J., Qin, S., Fan, X.-Y., ... Wang, L. (2019). The novel function of tumor protein D54 in regulating pyruvate dehydrogenase and metformin cytotoxicity in breast cancer. Cancer \& Metabolism, 7(1). https://doi.org/10.1186/s40170018-0193-4 


\section{Chapter 3: Citrate Synthase Regulation in Muscle during Hibernation}


Citrate synthase in hibernating Richardson's ground squirrels (Urocitellus richardsonii) displays altered functional properties

Stuart R. Green and Kenneth B. Storey

Institute of Biochemistry, Carleton University, 1125 Colonel By Drive, Ottawa ON, K1S 5B6, Canada

The following study is under consideration for publication in Cryobiology at the time of submission of this thesis 


\begin{abstract}
Hibernation is an incredible metabolic/physiological strategy employed by many mammals to cope with reduced food access during winter. Animals undergoing hibernation need to greatly reduce their metabolic rate and reshape their catabolic processes to survive on stored triglycerides. Citrate synthase (CS) is one of only two irreversible steps in the citric acid cycle (CAC) and forms an important regulatory checkpoint that gates the entry of acetyl-CoA formed in glycolysis or fatty acid catabolism into this critical central metabolic hub. This study investigated the regulation of citrate synthase in the muscle tissue of a small mammalian hibernator through comparison of functional and structural properties. The results demonstrated a significant decrease in the $V_{\max }$ of purified hibernating CS compared to the control euthermic enzyme (1.2-1.7 fold greater in the control) that was evident over a wide range of temperatures $\left(8,22\right.$ and $\left.37^{\circ} \mathrm{C}\right)$ that are encountered by the enzyme in hibernation. This was also reflected in the specific activity of the enzyme in crude muscle protein extracts. Analyzing the purified CS through immunoblotting demonstrated that the enzyme contained noticeably less lysine succinylation in the hibernating state (about $50 \%$ of euthermic levels) and this was correlated with an increase in total levels of SIRT5, the enzyme responsible for mediating desuccinylation in the mitochondria (2.2 fold increase). Taken together, the results of this study support the idea that CS is inhibited during hibernation in the ground squirrel skeletal muscle and that this alteration could be mediated by decreases in succinylation.
\end{abstract}

Keywords: citric acid cycle; ground squirrel; hibernation; citrate synthase; protein succinylation; metabolism 


\subsection{Introduction}

Hibernation is an important physiological and biochemical adaptation for many mammalian species that allows them to cope with the adverse conditions presented to them in the cold winters of temperate climates around the world. The principal threat to the survival of animals during the winter is the reduced access to food and water. While arboreal squirrel species have overcome this seasonal limitation through alterations in their behavior via caching food in hiding places to uncover when they need to feed, their subterranean cousins, the ground squirrels, go through astounding physiological changes during the winter to reduce their metabolism allowing them to survive on accumulated triglyceride stores during the winter months (van Breukelen \& Martin, 2015). The directly observable changes in the vital signs of the animal (reduction in heart rate, breathing rate, and body temperature) (Landau \& Dawe, 1958; Wang, 1979) are matched by significant alterations to their central metabolism that are controlled by mechanisms that exist apart from the mere effects one would expect from reduced body temperature. This is evidenced by the fact that $\mathrm{Q}_{10}$ is greatest between the temperatures of 20-30 ${ }^{\circ} \mathrm{C}$ for $\mathrm{O}_{2}$ consumption in hibernating animals but is lessened at lower temperature intervals $(0.1$ $\left.10{ }^{\circ} \mathrm{C}\right)$ (Geiser, 1988). The most notable of these changes is the shift from predominantly carbohydrate consumption over the summer months to a metabolism that is almost entirely fueled by triglycerides stored (can be over $50 \%$ of the animals body weight) in the adipose tissue (Wang \& Lee, 2011). The pronounced changes in metabolic demand led to questioning the role of central metabolic enzymes in controlling the rate of metabolism during hibernation and the flux of metabolic fuel through the mitochondria.

The citric acid cycle (CAC) is a central metabolic hub in all organisms that is located within the mitochondria critical to both catabolic and anabolic reactions. The CAC is most 
commonly associated with generating a substantial amount of the NADH needed to push electrons through the electron transport chain (ETC) in the mitochondria by catabolizing substrates fed into it from glycolysis and fatty acid oxidation. While the CAC is commonly thought of as primarily a catabolic cycle that yields energy from fuel sources, only two steps of the cycle are irreversible (citrate synthase and $\alpha$-ketoglutarate dehydrogenase) under biologically relevant concentrations of the substrates and products (Chinopoulos, 2013). Owing to the unidirectional flow through these steps, it is likely that these enzymes have preeminent regulatory significance as the reactions catalyzed by these proteins commit the substrates to more specific metabolic fates. While the importance of feedback inhibition on the functioning of the CAC is well documented, the requirement to spare fuel during hibernation likely means that a new homeostatic metabolic set point needs to be established through active measures. This has been previously suggested due to observations of reduced mitochondrial respiration during bouts of hibernation (Staples, 2014). A recent article published by the authors characterized the kinetic properties of the $\alpha$-ketoglutarate dehydrogenase complex (KGDC). This article demonstrated KGDC had a reduced affinity for coenzyme A (CoA), suggesting that in vivo there is likely reduced flux through this enzymatic complex during hibernation (Green \& Storey, 2020). This suggests that the CAC forms an underappreciated target for metabolic regulation in response to the metabolic changes accompanying hibernation.

Citrate synthase (CS) plays an important role in the CAC by catalyzing the addition of an acetyl group from acetyl-CoA to oxaloacetate and feeding substrates into the CAC in the mitochondrial matrix in an irreversible reaction (Chinopoulos, 2013). The overall reaction catalyzed by CS is as follows:

$$
\text { Acetyl-CoA }+ \text { Oxaloacetate } \rightarrow \text { Citrate }+ \text { CoA }
$$


Regulation of citrate synthase is thought to be controlled largely through allosteric regulation with high NADH concentrations resulting in feedback inhibition of the enzyme (Srere \& Matsuoka, 1972). In addition to feedback inhibition, CS regulation has more recently been demonstrated through post-translational modification (PTM). The post-translational modification takes the form of methylation at a specific lysine residue in the binding pocket (Lys 395 in the human sequence) and causes inhibition of the enzyme. The existence of a dedicated methyltransferase for CS known as CS lysine methyl transferase (CS-KMT or METTL12) emphasizes the importance of regulation of this enzyme in maintaining metabolic homeostasis (Małecki et al., 2017; Rhein, Carroll, Ding, Fearnley, \& Walker, 2017). Additionally, the effects of lysine acetylation in the Escherichia coli homolog of CS were determined to have an inhibitory effect on the activity of the enzyme (Venkat et al., 2019). Although no studies to date have looked at the role of lysine acetylation in the regulation of a eukaryotic homolog of CS, numerous high-throughput experiments have demonstrated acetylation on several sites within the human homolog of CS suggesting that a similar inhibitory function may exist in other animals (Choudhary et al., 2009; Mertins et al., 2013; Weinert et al., 2013; Zhao et al., 2010). Owing to the abundance of studies demonstrating various PTMs and the critical placement of CS in the CAC, alteration of CS structure in hibernation seems to be a plausible mechanism of potential regulation.

Reduction in the CAC and indeed in CS activity has been reported in a wide range of animals capable of metabolic rate depression. One such reduction was noted in a species of frog (Rana temporaria) where activity of this enzyme, and other mitochondrial enzymes, was lessened over the course of 4 months hypoxia in the skeletal muscle of the animal (St-Pierre \& Boutilier, 2001). Similarly, CS activities have been reported to be decreased by $40 \%$ in the 
hepatopancreas of an estivating terrestrial snail (Cepaea nemoralis) (Stuart, Ooi, \& Ballantyne, 1998). In the brown bear, citrate synthase and numerous other enzymes of the CAC has been demonstrated to be less abundant during hibernation in skeletal muscle (Chazarin et al., 2019). Oddly, this pattern seems somewhat variable between different hibernating species, with a hamster and a bat species actually demonstrating an increase in oxidative capacity through increased CS activity during hibernation in skeletal muscle (Kim et al., 2000; Wickler, Horwitz, \& S.Kott, 1987). These studies suggest that CS regulatory responses to hibernation vary between species and likely reflect the myriad roles the CAC plays in overall cellular metabolism and the rapidly changing metabolic conditions during hibernation.

Given the role of $\mathrm{CS}$ in catalyzing the reaction that brings substrates into the $\mathrm{CAC}$, the regulation of this enzyme was investigated. The study here describes the purification and characterization of the functional and structural properties of CS from euthermic and hibernating Richardson's ground squirrels.

\subsection{Materials and Methods}

\subsubsection{Animal Treatments}

Richardson's ground squirrels were captured near Calgary Alberta and transported to the University of Calgary to conduct the animal treatments. 40 male animals were collected near Calgary Alberta before being split into two different experimental groups. Animals were initially housed in separate rat cages in a room maintained on a $10 \mathrm{~h}$ light to $14 \mathrm{~h}$ dark photoperiod at 22 ${ }^{\circ} \mathrm{C}$ for 8 weeks during which they were fed ad libitum. Animals in the hibernation group were induced to hibernate by moving their cages to a room on a $16 \mathrm{~h}$ dark to $8 \mathrm{~h}$ light photoperiod at 4

${ }^{\circ} \mathrm{C}$. After $24 \mathrm{~h}$, food was withheld from the hibernating group although water was still provided. Any animals that did not enter into hibernation after the first week under these conditions were 
removed from the cold room and discontinued from the experiment. Animals that were in the hibernating state by the end of the week were kept at $4{ }^{\circ} \mathrm{C}$ for two additional days so that all animals were torpid for at least 2 days and the maximum time was 6-7 days. Euthermic animals were held at $22{ }^{\circ} \mathrm{C}$ for the entire time period. All animals were euthanized through decapitation and rapidly dissected for the tissues that were then frozen via immersion in liquid nitrogen. The tissues were shipped on dry ice from the University of Calgary to Carleton University where they were stored at $-80^{\circ} \mathrm{C}$ until use in the experiments. The protocols involving animals used in these experiments had received prior approval through the university Animal Care Committee and met the guidelines set by the Canadian Council on Animal Care.

\subsubsection{Protein Preparation and Purification}

Tissue samples were retrieved from storage at $-80^{\circ} \mathrm{C}$ and immersed in liquid nitrogen to keep cold. Muscle samples of the thigh were quickly weighed and homogenized in buffer A (50 mM Tris (pH 7.5), 2 mM EDTA, 2 mM EGTA, 20 mM $\beta$-glycerophosphate, 10\% glycerol v/v) in a 1:5 ratio w/v using a Polytron tissue homogenizer. A small spatula tip of phenylmethylsulfonylfluoride (PMSF) was included at the bottom of the test tube during homogenization to act as a protease inhibitor. Samples were promptly placed back on ice and then were placed into a pre-cooled $\left(4^{\circ} \mathrm{C}\right)$ refrigerated centrifuge and centrifuged for $30 \mathrm{~min}$ at $13,500 \times \mathrm{g}$. Following centrifugation, the supernatant was pipetted off and the rest was discarded.

The crude muscle extract were placed onto a Cibacron Blue chromatography column (3 $\mathrm{cm} \times 1.5 \mathrm{~cm}$ ) and allowed to flow though by gravity. The chromatography resin was rinsed with $15 \mathrm{~mL}$ of buffer A to elute unbound protein with 60-drop (approximately $2.1 \mathrm{~mL}$ ) fractions collected by a Gilson fractionator. Subsequently the CS was eluted from the column by applying 
$15 \mathrm{~mL}$ of a $1 \mathrm{mM}$ oxaloacetic acid (OAA)/coenzyme A (CoA) solution diluted in buffer A. This was based on a similarly described technique that found that these metabolites effectively eluted CS from ATP-agarose (Mukherjee \& Srere, 1976). 40-drop (approximately $1.3 \mathrm{~mL}$ ) fractions were collected following the application of the OAA/CoA solution. $1 \mu \mathrm{L}$ from each of these fractions were taken and assayed for CS activity. The top 5 fractions of CS activity were pooled together and saved for use in the next step of the purification.

The pooled fractions from the previous step were then subjected to removal of the metabolites (OAA and CoA) by filtration of the samples through a centrifugal concentrator unit (Amicon® Ultra 4 mL Centrifugal Filters, Millipore-Sigma, cat. No. UFC8010). During this procedure, the samples were spun in a 10-kDa cutoff protein concentrator unit for 20 minutes at $6,000 \times \mathrm{g}$ at $4{ }^{\circ} \mathrm{C}$. These samples were then diluted back to the original volume using buffer $\mathrm{A}$ and subjected to the same centrifugation step and dilution to elicit a fold dilution of the metabolites of at least 250 -fold from the original $1 \mathrm{mM}$. This step was critical as it was found that without removal of these metabolites, the protein would not adsorb to the carboxymethyl (CM) ion-exchange material.

After metabolite removal, the samples were loaded onto a $\mathrm{CM}$ cation exchange chromatography column $(7.5 \mathrm{~cm} \times 1.5 \mathrm{~cm})$ that was equilibrated in buffer $\mathrm{A}$ at $\mathrm{pH} 7.5$. The $\mathrm{CM}$ material was then subsequently rinsed with $15 \mathrm{~mL}$ of buffer $\mathrm{A}$ at $\mathrm{pH} 7.5$ to elute any unbound proteins. The $\mathrm{pH}$ of the buffer A was increased to 8.4 and a further $15 \mathrm{~mL}$ were applied to the column while collecting the eluate in 60-drop fractions. This helped remove most of the other proteins from the column without eluting CS owing to the very high isoelectric point of the enzyme. Purified CS was subsequently eluted from the column by use of a linear 0-2 $\mathrm{M} \mathrm{KCl}$ gradient while holding the $\mathrm{pH}$ of the buffer at 8.4. The top 5 fractions were pooled together and 
run through SDS-PAGE and stained in the gel using Coomassie stain to demonstrate the enzyme was purified to homogeneity.

\subsubsection{CS Assay}

CS was assayed according to methods based on the original technique developed by Mukherjee and Srere (Mukherjee \& Srere, 1976). This procedure exploits the production of CoA from acetyl CoA during the course of the CS mediated reaction and the free thiol groups of CoA subsequently rapidly reacts with 5,5'-dithiobis(2-nitrobenzoic acid) (DTNB) to form a dye which absorbs light strongly at $412 \mathrm{~nm}$ producing a visible yellow color (see Appendix A.1. for standard curve). This technique was modified slightly to make it suitable for use in a 96-well microplate as opposed to cuvettes. The reaction was carried out in a $50 \mathrm{mM}$ Tris buffer at $\mathrm{pH} 8.0$ at $22{ }^{\circ} \mathrm{C}$ with an initial concentration in each well of $0.1 \mathrm{mM}$ DTNB, $50 \mu \mathrm{M}$ acetyl CoA, and 1 mM OAA. $1 \mathrm{mU}$ of CS activity is defined as the amount of enzyme needed to produce $1 \mathrm{nmol}$ of CoA per minute under the aforementioned conditions. Reactions were performed at three different temperatures $\left(8^{\circ} \mathrm{C}, 22^{\circ} \mathrm{C}\right.$, and $\left.37^{\circ} \mathrm{C}\right)$, reflecting the wide range of body temperatures encountered by this animal during the hibernation cycle. Acetyl CoA was stored in $500 \mu \mathrm{L}$ aliquots at $-72{ }^{\circ} \mathrm{C}$ at neutral $\mathrm{pH}$ and thawed when needed to prevent degradation of the substrate (according to manufacturer protocols this will guarantee the stability for a minimum 6 months, individual batches of the substrate were used within $\sim 2$ months). All assays were performed using a Thermo Multiskan Spectrum microplate reading the absorbance at $412 \mathrm{~nm}$ every $21 \mathrm{~s}$ to monitor the reaction progress. When testing the inhibitory effects of NADH, suboptimal concentrations of the substrates (OAA) and acetyl CoA were used $(0.1 \mathrm{mM}$ and $0.0065 \mathrm{mM}$ respectively). 
For assays at $37^{\circ} \mathrm{C}$, an EchoTherm plate heater was used to pre-warm the reaction components (without one substrate) and the microplate reader's internal heater was set to $37^{\circ} \mathrm{C}$. The reaction was started by the addition of the missing substrate after the in-well temperature in the microplate reached $37^{\circ} \mathrm{C}$ (according to a thermistor probe reading) and the microplate reader's internal temperature reached $37^{\circ} \mathrm{C}$. For assays at $8{ }^{\circ} \mathrm{C}$, the microplate reader was placed in a VWR brand incubator (model \#2020) and allowed to cool to the required temperature. As before, the microplate was placed in the incubator and the assay was not started until the temperature inside the reaction mixture was at the appropriate temperature.

\subsubsection{Thermal Stability Assays}

To assess the thermal stability of the control and hibernating forms of CS, samples were incubated in a microplate heated to $60^{\circ} \mathrm{C}$ using the EchoTherm plate heater. Aliquots of the enzyme sample were pipetted out of the heated well at predetermined lengths of time (ranging from $1 \mathrm{~min}$ to $15 \mathrm{~min}$ ) and rapidly cooled on ice. These samples were then assayed for CS activity under the standard assay substrate concentrations and $\mathrm{pH}$ (described under CS Assay) at $22{ }^{\circ} \mathrm{C}$.

\subsubsection{Crude Homogenate Assays}

To determine the maximal CS activity in the euthermic and hibernating animal muscle, tissue homogenates from euthermic and hibernating animals were created as described before under the Protein Preparation and Purification section. Each of the homogenates were assayed using the Bradford method and then after having determined the concentration, the samples were subsequently diluted accordingly to $10 \mathrm{mg} / \mathrm{mL}$ of protein (Bradford, 1976). A portion of these samples was further diluted to $2 \mathrm{mg} / \mathrm{mL}$ and these were used to assess maximal activity of CS using the previously mentioned technique. The $10 \mathrm{mg} / \mathrm{mL}$ samples were diluted 1:1 in $2 \times$ SDS- 
PAGE loading buffer (100 mM Tris buffer, $\mathrm{pH} 6.8,4 \% \mathrm{w}: \mathrm{v}$ SDS, $20 \% \mathrm{v}: \mathrm{v}$ glycerol, $0.2 \% \mathrm{w}: \mathrm{v}$ bromophenol blue) with $10 \% \mathrm{v}: \mathrm{v} \beta$-mercaptoethanol and immersed in boiling water for 5 minutes. These samples were then used for western blotting to assess the relative levels of CS and SIRT5 in each of the samples (see Western Blotting section).

\subsubsection{Western Blotting}

Western blotting was performed to determine the relative levels of various posttranslational modifications (PTMs) in the purified samples from the euthermic and hibernating muscle. Purified CS samples were first concentrated $8 \times$ to improve the signal for assessing the PTMs by use of centrifugal concentrator units as described under Protein Preparation and Purification. Protein samples were diluted as appropriate to ensure that equal amounts of CS were loaded onto the Western blots as assessed by Coomassie stained test blots. Approximately $0.79 \mu \mathrm{g}$ of the CS protein was found to be sufficient to give a strong signal without oversaturating the Coomassie stain and this amount was therefore used going forward. Samples were run through a $10 \%$ polyacrylamide gel using a gel electrophoresis apparatus (BioRad Mini Protean III $($ ) at $180 \mathrm{~V}$ for $65 \mathrm{~min}$. All samples were run alongside a $10.5-175 \mathrm{kDa}$ PiNK Plus pre-stained protein ladder (FroggaBio \# PM005-0500) for size comparison. The proteins were then transferred to an Immobilon-P polyvinylidene fluoride (PVDF) membrane (Millipore \# IPVH00010) pre-wetted in methanol through a BioRad Mini Trans-Blot ${ }^{\circledR}$ Electrophoretic Transfer Cell transfer apparatus set to $200 \mathrm{~mA}$ for 90 minutes. Both the electrophoresis and membrane transfer were powered using a BioRad Power Pac 200®. After the transfer, the membranes were incubated with the primary detection antibody overnight. The immunoblots quantifying the amounts of CS and SIRT5 were run with $25 \mu \mathrm{g}$ of protein from tissues homogenates and blocked by immersing it in a $2 \% \mathrm{w}: \mathrm{v}$ skim milk powder solution dissolved in 
TBST (20 mM Tris base, $\mathrm{pH} 7.6,140 \mathrm{mM} \mathrm{NaCl}, 0.05 \% \mathrm{v}: \mathrm{v}$ Tween-20) for $30 \mathrm{~min}$ before washing $3 \times 5$ min with TBST and then incubated overnight with anti-citrate synthase antibody (GeneTex \# GTX110624) or anti-SIRT5 antibody (GeneTex \# GTX117825) both diluted 1:1000 $\mathrm{v}: \mathrm{v}$ in TBST. The following antibodies were used for the detection of PTMs on the CS through overnight application:

1. Anti-phosphorylated serine (Invitrogen \# 61-8100)

2. Anti-phosphorylated threonine (Invitrogen \# 71-8200)

3. Anti-phosphorylated tyrosine (Invitrogen \# 13-6600)

4. Anti-methyl lysine (StressMarq Biosciences Inc. \# SPC-158F

5. Anti-acetyl lysine (Santa Cruz Biotechnology \# SC-8663)

6. Anti-succinyl lysine (PTM Biolabs \# PTM-401)

7. Anti-malonyl lysine (PTM Biolabs \# PTM-901)

8. Anti-glutaryl lysine (PTM Biolabs \# PTM-1151)

9. Anti-pan-ADP-ribose binding reagent (EMD Millipore \# MABE1016)

10. Anti-nitrosyl cysteine (Abcam \# ab50185)

After removing the primary antibody from the blots, the blots were then further washed 3 $\times 5$ min using TBST to remove unbound antibody from the membrane. The membranes were then left to incubate with rocking in 1:8000 diluted secondary anti-IgG antibody conjugated with horseradish peroxidase, these antibodies were raised against either rabbit or mouse IgG depending on the primary antibody. Signal detection was performed using an enhanced 
chemiluminescence (ECL) protocol and visualized using a ChemiGenius Bioimaging System (Syngene, Frederick, MD). Blots were subsequently stained using Coomassie brilliant blue and pictures were taken using the same system as before. The CS protein bands in the Coomassie stained blots were used to normalize the ECL signal for PTM blotting. A group of stably expressed proteins were used as loading controls for the CS and SIRT5 signal in the immunoblots quantifying these proteins in unenriched samples (Eaton et al., 2013). Images were analyzed using GeneTools software (version 4.3.8.0).

\subsubsection{Bioinformatics Resources}

The CS sequence of the closest sequenced relative of the Richardson's ground squirrel (the 13-lined ground squirrel, Ictidomys tridecemlineatus, GenBank Accession \# KAG3291618.1) was aligned to the human sequence using the Clustal Omega multiple sequence alignment tool (Appendix A.2.) (Sievers et al., 2011). This sequence alignment demonstrates a high degree of sequence similarity between the human and ground squirrel CS sequence. Using the PhosphoSitePlus online resource (https://www.phosphosite.org/proteinAction.action?id=13876\&showAllSites=true), candidate lysine succinylation sites were identified based on high-throughput data from the human sequence, all of these lysine sites were conserved in the ground squirrel sequence. These sites were plotted next to a diagram of the CS sequence from 13-lined ground squirrel mapped with the location of functionally significant residues sorted using the National Center for Biotechnology Information (NCBI) conserved domain database (Marchler-Bauer et al., 2015, 2017). The isoelectric point for I. tridecemlineatus CS was calculated using the Expasy Compute pI/MW online tool (https://web.expasy.org/compute_pi/). 
Protein modelling was performed by using the I-TASSER Protein Structure \& Function Predictions server (Roy, Kucukural, \& Zhang, 2010; Yang et al., 2014; Y. Zhang, 2008). The best model of the protein complexed with an analog for the transition state of the substrates (Scitryldethia coenzyme A) was rendered using PyMOL software (https://pymol.org/2/). In PyMOL, the known mammalian lysine succinylation sites were identified and labeled to visualize the positioning of potential succinylation sites on the 13-lined ground squirrel CS sequence.

\subsubsection{Statistical Analysis}

Raw enzyme kinetic data was analyzed using a microplate analysis program (MPA) and enzyme kinetic parameters were calculated from activity data using Kinetics 3.51 software using least squares regression for data modelling (Brooks, 1992; Brooks, 1994). Where applicable RBioplot statistical software was used in data analysis to generate graphs and perform Student's t-test or analysis of variance (ANOVA) combined with a Tukey's post-hoc test (Zhang \& Storey, 2016). Data was determined to be statistically different between euthermic and hibernating when the $\mathrm{p}<0.05$.

\subsection{Results}

\subsubsection{Purification}

CS was purified to homogeneity in a two-step chromatographic protocol. The first step utilized a dye affinity chromatography (Cibacron Blue) column. Elution from the Cibacron Blue column was accomplished addition of $1 \mathrm{mM} \mathrm{OAA}$ and CoA into the solution. This was found to be a very effective method of purifying the enzyme since many of the proteins that adhered to the resin material did not elute using these concentrations of the metabolites, while eluting most of the CS bound to the column (62.6\% yield for control and 61.5\% for hibernating) (Table 3.1.). 
Following dilution of the metabolites added for elution of the CS from the Cibacron blue column, the enzyme was adsorbed to a cation exchange chromatography column, carboxymethyl (CM) sepharose equilibrated in a $\mathrm{pH} 7.5$ buffer. Owing to the high isoelectric point (pI) of CS (theoretical $\mathrm{pI}$ of 8.58 based on the sequence of Ictidomys tridecemlineatus), the enzyme remained bound to the column matrix even after increasing the $\mathrm{pH}$ of the buffer to 8.4 while consequently removing most of the impurities. Elution from this column was performed using an increasing $\mathrm{KCl}$ gradient from $0-2 \mathrm{M} \mathrm{KCl}$. The final enzyme preparations consisted of a single protein band located at the same molecular weight as the commercially available CS demonstrated through Coomassie staining of a 10\% SDS-PAGE gel (Fig. 3.1.).

\subsubsection{Enzyme Kinetics}

Analysis of maximal CS activity in tissue protein extracts demonstrated a significant decrease in the hibernating samples activity per milligram protein that remained consistent across the three temperatures tested (hibernating $72 \%$ at $8{ }^{\circ} \mathrm{C}, 80 \%$ at $22{ }^{\circ} \mathrm{C}, 70 \%$ at $37{ }^{\circ} \mathrm{C}$ ) (Fig. 3.2.). Kinetic parameters were assessed in protein preparations purified from euthermic and hibernating animals. These tests demonstrated a similar trend in $V_{\max }$ activity with the hibernating demonstrating a lower $V_{\max }$ again across the three temperatures tested, $8{ }^{\circ} \mathrm{C}, 22{ }^{\circ} \mathrm{C}$ and $37^{\circ} \mathrm{C}(77 \%, 84 \%$, and $59 \%$ of the euthermic value respectively) (Table 3.2.). Reduced temperature relative to standard body temperature $\left(37^{\circ} \mathrm{C}\right)$ was noted to cause a significant decrease in the $K_{m} \mathrm{OAA}$ in both the euthermic and hibernating enzyme samples. This effect was first noticeable at $22{ }^{\circ} \mathrm{C}$ for the hibernating ( 0.24 -fold change) but only noticeable in the euthermic sample at $8{ }^{\circ} \mathrm{C}(0.31$ fold change). The effects of temperature were not statistically different for the hibernating form of the enzyme in regards to the $K_{m}$ acetyl CoA even though in the euthermic form this was significantly decreased at $22{ }^{\circ} \mathrm{C}$ relative to $37{ }^{\circ} \mathrm{C}(0.4$-fold) although 
no change was noticeable between $37^{\circ} \mathrm{C}$ and $8{ }^{\circ} \mathrm{C}$. The $K_{m}$ acetyl-CoA was not significantly different between the euthermic and hibernating enzyme samples for any of the temperatures measured. Additionally, the $K_{m}$ OAA was significantly lower in the hibernating form of the enzyme than the euthermic at $22{ }^{\circ} \mathrm{C}$, although no significant difference was noted between the euthermic and the hibernating $K_{m} \mathrm{OAA}$ at $8^{\circ} \mathrm{C}$ or $37^{\circ} \mathrm{C}$ even though this trend appeared present at $8{ }^{\circ} \mathrm{C}$ this was not significant $(\mathrm{p}=0.11)$. NADH was tested as an inhibitor of the reaction at 22 ${ }^{\circ} \mathrm{C}$ and was noted to differentially affect the euthermic and the hibernating enzyme. The NADH $\mathrm{I}_{50}$ value (concentration resulting in half-maximal inhibition) for CS from the hibernating animal was only $55.5 \%$ the same value as the euthermic control.

\subsubsection{Protein Stability}

Thermal stability of the euthermic and hibernating enzyme appears to be comparable between the purified enzyme samples. Thermal stability was tested by exposing the enzymes to $60{ }^{\circ} \mathrm{C}$ for varying lengths of time and then assaying the enzyme under standard assay conditions at $22{ }^{\circ} \mathrm{C}$. The point of inflection, the midpoint between the native state and complete denaturation was calculated with respect to time as $2.5 \pm 0.15 \mathrm{~min}$ for control CS and $2.9 \pm 0.19$ min for the hibernating CS and were not statistically different at the $\mathrm{p}<0.05$ level using Student's $t$-test (Fig. 3.3.).

\subsubsection{Western Blots for Post-translational Modifications}

Using western blots to analyze the relative level of various post-translational modifications (PTMs) on purified CS samples demonstrated a significant decrease in the relative degree of lysine succinylation on CS (0.49-fold) in the hibernating compared to the euthermic samples. Additionally malonyl-Lys seemed to be found at lower levels in the hibernating 
samples compared to the control euthermic, although this was not statistically different at the $\mathrm{p}<$ 0.05 level $(\mathrm{p}=0.072)$.

\subsubsection{CS and SIRT5 Levels}

The total levels of CS were investigated in the euthermic and hibernating samples using immunoblotting. The results showed a single band on the membrane in the same position as those in the purified samples (Appendix A.3.). No significant changes were found in the amount of CS when comparing the relative levels between the two experimental groups (Fig. 3.5.). Owing to the decrease in levels of succinylation observed in the hibernating muscle CS, SIRT5, the protein responsible for de-succinylation in the mitochondria, was hypothesized to be upregulated during hibernation. Using immunoblots on crude protein extracts from the euthermic and hibernating muscle samples, this was confirmed to be the case with levels of SIRT5 at 2.19-fold higher in the hibernation as compared to the control samples (Fig. 3.5.).

\subsubsection{Bioinformatics Analysis}

Use of the PhosphoSitePlus ${ }^{\circledR}$ online resource demonstrated abundant evidence for the lysine succinylation of human CS

(https://www.phosphosite.org/proteinAction.action?id=13876\&showAllSites=true). When these sites were checked against the ground squirrel sequence it was found that all of them were conserved (Appendix A.2.). When the positions of these known succinylation sites were compared to a conserved domain map of CS, none were found to be located in the active site although one was found on a residue relating to the dimer interface (Fig. 3.6.). 


\subsection{Discussion}

CS is a key enzyme of the CAC that is necessary for condensing acetyl CoA units generated by the pyruvate dehydrogenase complex with oxaloacetate to create citrate. The reaction catalyzed by CS is considered to be one of only two irreversible reactions in the CAC and therefore serves as an important step in the regulation of central metabolism as it has a highly negative free energy of $-36.6 \mathrm{~kJ} / \mathrm{mol}$ (Chinopoulos, 2013; Li, Wu, Qi, \& Beard, 2011). Citrate synthase is a crucial step in regulating the overall rate of the CAC and metabolism and its activity is commonly used as a marker for measuring mitochondrial respiration and oxidative capacity (Larsen et al., 2012; Vigelsø, Andersen, \& Dela, 2014). Owing to the position of CS at the entry of substrates into the CAC and also the profound decreases in metabolic activity during hibernation, the role of CS regulation during hibernation was investigated (Wang \& Lee, 2011).

The purification of CS from muscle tissue followed a relatively simple procedure consisting of two chromatography steps. Both of the chromatographic materials are relatively non-specific in their ability to bind proteins. However, the steps taken to elute CS are specific enough to eliminate the contaminant proteins while retaining a high percentage of the original CS activity. The first step employed Cibacron Blue agarose, a dye based material that binds a wide variety of proteins often that have a nucleotide-like binding site (Subramanian \& Ross, 1984). Elution from this resin was based off a comparable technique described by Mukherjee and Srere (Mukherjee \& Srere, 1976) wherein a solution containing CoA and ATP is used to elute CS. After removing the CoA and ATP using centrifugal concentration, CS could adsorb to CM cation exchange resin at $\mathrm{pH}$ 7.5. This step proved to be highly valuable in separating CS from the other proteins from the previous step due to the very high isoelectric point of mammalian CS ( 8.58 in the 13-lined ground squirrel). By comparison the average isoelectric point of most 
eukaryotic proteins is below 7.0 with few over 8.0 (Kozlowski, 2017). For this reason, increasing the $\mathrm{pH}$ of the cation exchange column up to 8.4 removed most of the other proteins that adsorbed to the material. Increasing $\mathrm{KCl}$ concentration allowed for elution of a highly enriched $\mathrm{CS}$ fraction. The methods described here demonstrate how chromatographic methods with a relatively low adsorption specificity can be utilized to allow for a high degree of purification when an understanding of the molecular properties of the target is applied to developing an elution protocol.

Analysis of the kinetic parameters of the purified CS enzyme demonstrated a significant decrease in the maximal activity (Table 3.2.). This was observed across a wide range physiological temperatures that are experienced by the ground squirrel over the course of hibernation. To provide greater physiological context to this finding, the CS specific activity of whole tissue homogenates from the control euthermic and hibernating groups were compared and the same pattern held true with the euthermic displaying higher levels of CS activity when normalized against total soluble protein concentration (Fig. 3.2.). This was in spite of the relative levels of CS remaining constant in hibernating muscle (Fig. 3.5.). Additionally NADH demonstrated greater inhibitory potency at $22{ }^{\circ} \mathrm{C}$ in the hibernating CS compared with the euthermic group. While the effects of hibernation on the redox balance of $\mathrm{NAD}^{+} / \mathrm{NADH}$ in the mitochondrial matrix are unknown, the concentration of NADH is estimated under normal conditions to be approximately $3.5 \mathrm{mM}$ in porcine heart mitochondria, meaning that the $I_{50}$ values for the euthermic and hibernating CS ( $4.5 \pm 0.6$ and $2.5 \pm 0.4 \mathrm{mM}$ respectively) are likely physiologically relevant (Blinova et al., 2005). Since the $I_{50}$ values are very close to the approximate concentrations of NADH found in the mitochondria, these observations likely have profound implications for the function of hibernating CS in vivo and the inhibition is therefore 
not merely an artefact of using in vitro concentrations of NADH that would vastly exceed physiological levels. While the $K_{m}$ oxaloacetate (OAA) was higher in the euthermic than in the hibernating at $22{ }^{\circ} \mathrm{C}$, this difference was not observed at any other temperature. Additionally, the higher $V_{\max }$ and NADH $I_{50}$ in the control likely mean that this effect is neither significant at euthermic temperatures nor when the animal is at its deepest state of torpor when its body temperature sinks to $2-3{ }^{\circ} \mathrm{C}$ (Wang, 1979).

No significant trend in the acetyl CoA $K_{m}$ were noted between 8 and $37^{\circ} \mathrm{C}$ for either the euthermic or hibernating form of the enzyme, this was in contrast to the OAA $K_{m}$ that decreased noticeably at lower temperatures for both the euthermic and the hibernating forms of the enzyme. The reduction of $V_{\max }$ observed in the hibernating form of the enzyme combined with the effects of decreased body temperature place a firm upper limit on the flow of substrates through CS during hibernation. This is contrasted by the decrease in $K_{m} \mathrm{OAA}$ at lower temperatures that may help to ensure basal levels of CS activity are maintained in the possible event OAA levels becoming depleted over hibernation. This idea is supported by the observation that at $22{ }^{\circ} \mathrm{C}$ the $K_{m}$ OAA was actually higher in the euthermic CS than the hibernating, implying that the euthermic form is more resistant to the effects of the reduced temperature than the hibernating since it retains the higher $K_{m}$ observed at $37^{\circ} \mathrm{C}$. These changes in sensitivity to OAA are likely to have real consequences in mitochondrial metabolism since the $K_{m}$ values measured here are close to the estimated concentrations of OAA in the mitochondria (Nazaret, Heiske, Thurley, \& Mazat, 2009; Siess, Kientsch-Engel, \& Wieland, 1984). Since the low-temperature dependent reduction in $K_{m}$ OAA occurs at a higher temperature in the hibernating form of the enzyme, this may suggest that CS remains comparatively active during the early stages of hibernation (torpor can start as early as September when body temperatures are around $\sim 20^{\circ} \mathrm{C}$ (Wang, 1979), 
especially in the absence of the feedback inhibitor NADH. Although no studies have shown changes in the $\mathrm{NAD}^{+}: \mathrm{NADH}$ ratio during hibernation, it has been suggested elsewhere (Klug \& Brigham, 2015) that this ratio may be increased during hibernation and the increased sensitivity of CS NADH inhibition may therefore be critical in compensating for reduced mitochondrial NADH levels to ensure a reduction in CS activity. Complete shutdown of the CAC would not be beneficial to the animal since, although glycolysis almost completely ceases in the muscle tissues, the breakdown of fatty acids is still needed to generate some acetyl CoA for the CAC to provide sufficient energy for the maintenance of essential metabolic processes. The increased sensitivity to NADH inhibition and the lower $V_{\max }$ observed in the hibernating form of CS helps to limit the upper rate of how fast acetyl CoA can be incorporated into the CAC. These findings are reminiscent of other studies that have presented varied accounts of the nature of CS regulation in hibernation, with some demonstrating an increase in activity and others a decrease (Chazarin et al., 2019; Kim et al., 2000; Stuart et al., 1998). Taken together these findings suggest a complex role of CS regulation in relation to the changing metabolic demands that occur over the hibernation season.

Owing to the differences in kinetic parameters seen between the euthermic and hibernating CS, most notably the decrease $V_{\max }$ in the purified CS samples, structural differences between the two forms were proposed to be the source of these changes. The purified samples were probed with various antibodies recognizing different PTMs in order to assess the relative degree of modification for each respective target. The results indicated few changes between the euthermic and hibernating samples except for a decrease in lysine succinylation. Lysine succinylation is not as well characterized as other more familiar modifications such as phosphorylation or acetylation and its effects are not as well studied. It is likely that 
succinylation imparts a significant change in the functionality of proteins, perhaps even more so than methylation or acetylation, owing to its bulkier size ( $100 \mathrm{Da})$ (Zhang et al., 2011). Highthroughput mass spectrometry data has demonstrated that lysine succinylation is present on several lysine sites in the human homolog of CS (https://www.phosphosite.org/proteinAction.action?id=13876\&showAllSites=true). Not much is known about how succinylation of these sites affects CS activity and indeed protein modelling demonstrated that none of these sites were very close to the active site pocket (Fig. 3.6.). Interestingly, although lysine methylation is a known PTM of CS whose addition by a specific lysine methyl transferase (CS-KMT) on the Lys-395 residue causes inhibition in the human sequence, no changes in lysine methylation were observed here (Małecki et al., 2017). Although this Lys-395 residue is known to be important in regulating substrate binding, it is unlikely to be a factor here since succinylation of this residue would likely induce greater inhibition than methylation by causing steric hindrance in the active site, as opposed to the activation that was seen in the more succinylated form. The large number of known succinylation sites from high throughput studies in the human homolog of the protein and also the possibility that other residues may be succinylated in the ground squirrel make it difficult to predict which ones if any are responsible for the changes in kinetic parameters observed here. Mapping of the known lysine succinylation sites revealed few near the substrate binding site however, the K450 site was predicted to be in the dimer interface region (Fig. 3.6.). This suggests the possibility that alterations in the tertiary structure of the CS dimer could be responsible for the increased activity in the higher succinyl form. In the mitochondria, CS is also hypothesized to participate in semistable complexes known as metabolons within the highly protein dense mitochondrial matrix, particularly with malate dehydrogenase, to aid in the transport of metabolites between enzymes 
in the $\mathrm{CAC}$ in a process known as substrate channeling (Bulutoglu, Garcia, Wu, Minteer, \& Banta, 2016; Huang, Huber, Wang, Minteer, \& McCammon, 2018; Tompa, Batke, Ovadi, Welch, \& Srere, 1987). Indeed, the methylation of CS due to CS-KMT has been proposed to alter the nature of the intermolecular forces holding the metabolons together (Rhein et al., 2017). This leads to additional unanswered questions about how the introduction or removal of large succinyl moieties onto the surface of CS would affect its interactions with other proteins and overall what consequences this might entail for metabolism.

The difference in succinylation in CS in this animal in particular is interesting since a previously published study noted a change in succinylation in a mitochondrial protein in this same animal and tissue during hibernation. In that study the $\alpha$-ketoglutarate dehydrogenase E1 subunit (KGDC E1) was noted to be significantly increased in succinyl lysine levels compared to the control euthermic levels (Green \& Storey, 2020). This was suggested in that publication to potentially be the result of changes in the functionality of the KGDC complex itself since this complex is responsible for creating succinyl-CoA and perhaps may even have a role in directly succinylating target proteins (Gibson et al., 2015). The decrease in CS succinylation in the current study is therefore somewhat surprising owing to the increase in the succinylation activity of the KGDC during hibernation, nonetheless, it is possible that there may merely be a greater degree of self-succinylation of the KGDC, thereby reducing the levels of succinyl-CoA available for other targets (such as CS). In the mitochondria, there are several sirtuin proteins that are best known for their deacetylation function (Lombard, Tishkoff, \& Bao, 2011). In more recent years, the role of SIRT5 specifically has become better elucidated as a general-purpose deacylation enzyme that controls the levels of several protein-modifying acyl groups including succinyl lysine (Park et al., 2013). Owing to the notable decrease in lysine succinylation during 
hibernation on CS, the levels of SIRT5 in muscle protein extracts were examined. These results demonstrated that SIRT5 was indeed decreased in expression during hibernation, strongly hinting that SIRT5 is the agent responsible for the changes seen here in lysine succinylation. This is consistent with what is known about the succinylome since previous studies have demonstrated in mice that knockouts of SIRT5 rapidly lead to hypersuccinylation (Rardin et al., 2013). Additionally it has been recently demonstrated that succinyl-CoA ligase is phosphorylated during hibernation in the liver of a related ground squirrel species, though the effect that this may have on mitochondrial succinyl-CoA levels and whether this occurs in the muscle tissue is unknown (Mathers \& Staples, 2019). The mitochondria is a metabolic hub that is beginning to be understood to have a large variety of PTMs that likely contribute to metabolic regulation. This is especially true in regards to acylation, since the mitochondria is a locus where numerous processes driven by acylated CoA derivatives occur including fatty acid oxidation and the CAC.

The results of this study have demonstrated that CS activity is reduced in Richardson's ground squirrel muscle during hibernation, and that these changes are not due to a decrease in the overall amount of the protein present. This is in line with a growing body of literature that has suggested that the transition to hibernation and the concomitant reduction in metabolic rate is a consequence of more than just the decrease in body temperature, but changes to the structure of key metabolic enzymes aid in metabolic reduction. Of particular interest for comparison is a recently published study on the effects of hibernation on the KGDC activity which demonstrated a significant decrease in substrate affinity for acetyl CoA, suggesting a decrease in flux through the complex during hibernation (Green \& Storey, 2020). Taken together with this earlier study, the results presented here demonstrate that restriction on the rate of flow through the CAC is a 
likely feature of hibernation in the ground squirrel muscle. While no acetylation changes were observed in this study on CS, it is thought that control over mitochondrial respiration at complex IV in the electron transport system is mediated by enhanced acetylation during torpor in the liver (Mathers \& Staples, 2019). This observation is somewhat counterintuitive since SIRT activity is generally associated with deacetylation and in muscle tissue during torpor total SIRT activity is noted to increase (Rouble \& Storey, 2015). It could be that different strategies are taken in the liver and in the skeletal muscle of ground squirrels to affect a reduction in metabolic rate.

\subsection{Conclusion}

The results seen in this study demonstrate that during hibernation the activity of citrate synthase is reduced across a wide range of temperatures that would be encountered over the winter. The decrease in activity was noted both in purified samples as well as in crude protein extracts of the skeletal muscle. The decrease in activity was accompanied with an increase in sensitivity to inhibition by NADH, a down-stream product of the CAC. Immunoblotting demonstrated a decrease in the relative levels of lysine succinylation and this was correlated with an increase in total levels of SIRT5 in the crude protein extracts. Taken together the results support the idea that inhibition of CS is an important feature in the metabolic regulation of the CAC during hibernation and that this change may be elicited by decreases in lysine succinylation during hibernation.

\section{Acknowledgements}

The research performed in this article was supported by a Discovery Grant (6793) from the Natural Sciences and Engineering Research Council (NSERC) of Canada to KB Storey. KB Storey holds the Canada Research Chair in Molecular Physiology. SR Green was awarded and NSERC Canada Graduate Scholarship at the doctoral level and an Ontario Graduate Scholarship 
during the time of the research. The authors thank JM Storey for her time and insights in the editing of the manuscript.

\section{Disclosure}

The authors declare no competing interests financial or otherwise in the contents and conclusions of the manuscript. 
Table 3.1. Representative purifications of citrate synthase from control euthermic and hibernating Richardson's ground squirrel skeletal muscle.

\section{Euthermic}

\begin{tabular}{|c|c|c|c|c|c|}
\hline Step & $\begin{array}{l}\text { Total } \\
\text { Protein }(\mathrm{mg})\end{array}$ & $\begin{array}{l}\text { Total Activity } \\
(\mathrm{mU})\end{array}$ & $\begin{array}{l}\text { \% Yield from } \\
\text { Crude }\end{array}$ & $\begin{array}{l}\text { Specific Activity } \\
(\mathrm{mU} / \mathrm{mg})\end{array}$ & $\begin{array}{l}\text { Fold } \\
\text { Purification }\end{array}$ \\
\hline Crude & 26.89 & 10338 & - & 384.4 & - \\
\hline $\begin{array}{l}\text { Cibacron Blue (1 } \\
\text { mM OAA/CoA ) }\end{array}$ & 0.490 & 6470 & 62.6 & 13192 & 34.3 \\
\hline $\mathrm{CM} 0-2 \mathrm{M} \mathrm{KCl}$ & 0.088 & 2791 & 27.0 & 31714 & 82.5 \\
\hline \multicolumn{6}{|l|}{ Hibernating } \\
\hline Step & $\begin{array}{l}\text { Total } \\
\text { Protein }(\mathrm{mg})\end{array}$ & $\begin{array}{l}\text { Total Activity } \\
(\mathrm{mU})\end{array}$ & $\begin{array}{l}\text { \% Yield from } \\
\text { Crude }\end{array}$ & $\begin{array}{l}\text { Specific Activity } \\
(\mathrm{mU} / \mathrm{mg})\end{array}$ & $\begin{array}{l}\text { Fold } \\
\text { Purification }\end{array}$ \\
\hline Crude & 38.27 & 12666 & - & 331 & - \\
\hline $\begin{array}{l}\text { Cibacron Blue (1 } \\
\text { mM OAA/CoA ) }\end{array}$ & 1.47 & 7792 & 61.5 & 5312 & 16.1 \\
\hline CM 0-2 M KCl & 0.12 & 2739 & 21.6 & 22545 & 68.1 \\
\hline
\end{tabular}


Table 3.2. Comparison of the kinetic parameters of control euthermic and hibernating CS from skeletal muscle of Richardson's ground squirrels. Data represent the mean of $n=4$ determinations $( \pm \mathrm{SEM}) .{ }^{*}$ ' represents a hibernating parameter that is statistically different from the corresponding euthermic control by Student's t-test $(p<0.05)$. Where the hibernating mean is statistically different from the euthermic, the fold change is given in the right-most column to give context to the magnitude of the change. Parameters within the same experimental group (euthermic or hibernating) were compared across a range of temperatures using ANOVA tests with Tukey's post hoc tests. Values were assigned to groups by letter $(\mathrm{a} / \mathrm{b} / \mathrm{c})$ with parameters statistically different labeled with different letters $(\mathrm{p}<0.05)$.

\begin{tabular}{|c|c|c|c|}
\hline Parameter & Euthermic & Hibernating & Fold Change \\
\hline \multicolumn{4}{|l|}{$8^{\circ} \mathrm{C}$} \\
\hline $\mathrm{V}_{\max }(\mathrm{mU} / \mathrm{mg})$ & $17400 \pm 1300^{\mathrm{a}}$ & $13400 \pm 400^{\mathrm{a} *}$ & 0.77 \\
\hline Oxaloacetate $K_{m}(\mu \mathrm{M})$ & $3.88 \pm 0.3^{\mathrm{a}}$ & $2.47 \pm 0.6^{\mathrm{a}}$ & NA \\
\hline Acetyl CoA $K_{m}(\mu \mathrm{M})$ & $2.49 \pm 0.3^{\mathrm{ab}}$ & $2.06 \pm 0.3^{\mathrm{a}}$ & NA \\
\hline \multicolumn{4}{|l|}{$22{ }^{\circ} \mathrm{C}$} \\
\hline $\mathrm{V}_{\max }(\mathrm{mU} / \mathrm{mg})$ & $34700 \pm 600^{b}$ & $29100 \pm 900^{\mathrm{b} *}$ & 0.84 \\
\hline Oxaloacetate $K_{m}(\mu \mathrm{M})$ & $9.61 \pm 0.7^{b}$ & $3.11 \pm 0.5^{a *}$ & 0.32 \\
\hline Acetyl-CoA $K_{m}(\mu \mathrm{M})$ & $1.66 \pm 0.5^{\mathrm{a}}$ & $2.63 \pm 0.4^{\mathrm{a}}$ & NA \\
\hline $\mathrm{I}_{50} \mathrm{NADH}(\mathrm{mM})$ & $4.5 \pm 0.6$ & $2.5 \pm 0.4^{*}$ & 0.56 \\
\hline \multicolumn{4}{|l|}{$37^{\circ} \mathrm{C}$} \\
\hline $\mathrm{V}_{\max }(\mathrm{mU} / \mathrm{mg})$ & $59000 \pm 2600^{c}$ & $35000 \pm 2000^{\mathrm{c} *}$ & 0.59 \\
\hline Oxaloacetate $K_{m}(\mu \mathrm{M})$ & $12.4 \pm 1.4^{\mathrm{b}}$ & $12.7 \pm 0.6^{b}$ & NA \\
\hline Acetyl-CoA $K_{m}(\mu \mathrm{M})$ & $4.14 \pm 0.4^{b}$ & $3.59 \pm 0.7^{\mathrm{a}}$ & NA \\
\hline
\end{tabular}


A

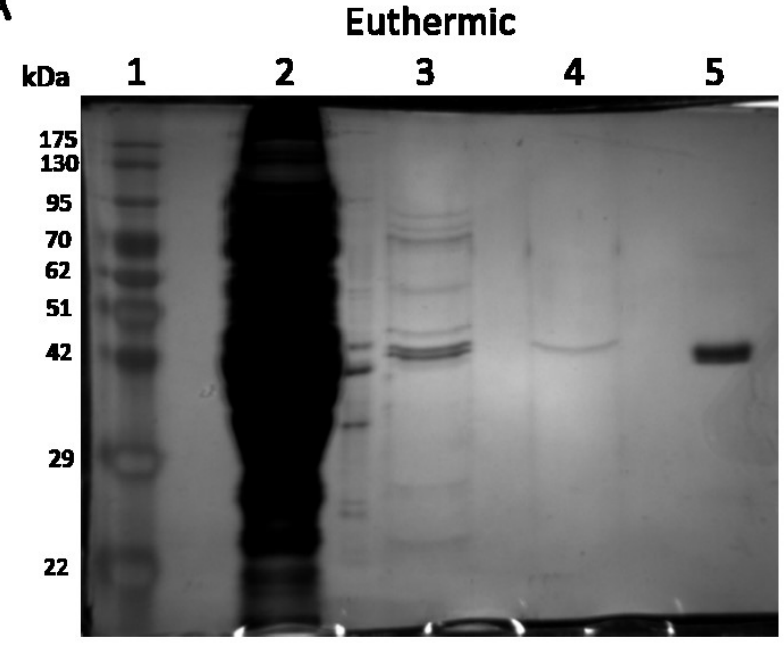

B

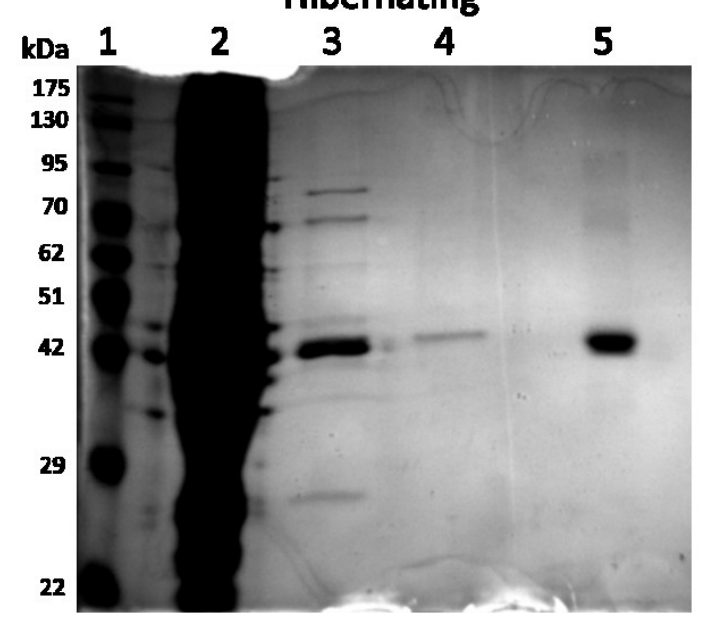

Fig. 3.1. Purification scheme of citrate synthase (CS) from control euthermic (A) and hibernating (B) Richardson's ground squirrel muscle visualized through 10\% acrylamide SDS-PAGE and stained with silver staining. Lanes are as follows: 1: Molecular weight ladder, 2: Crude homogenate, 3: pooled fractions after elution from Cibacron Blue with oxaloacetate and coenzyme A, 4: Pooled fraction from elution off carboxymethyl sepharose at $\mathrm{pH} 8.4$ over a linear 0-2 M KCl gradient, 5: Commercially purified CS from porcine heart (Sigma-Aldrich \#C3260). 


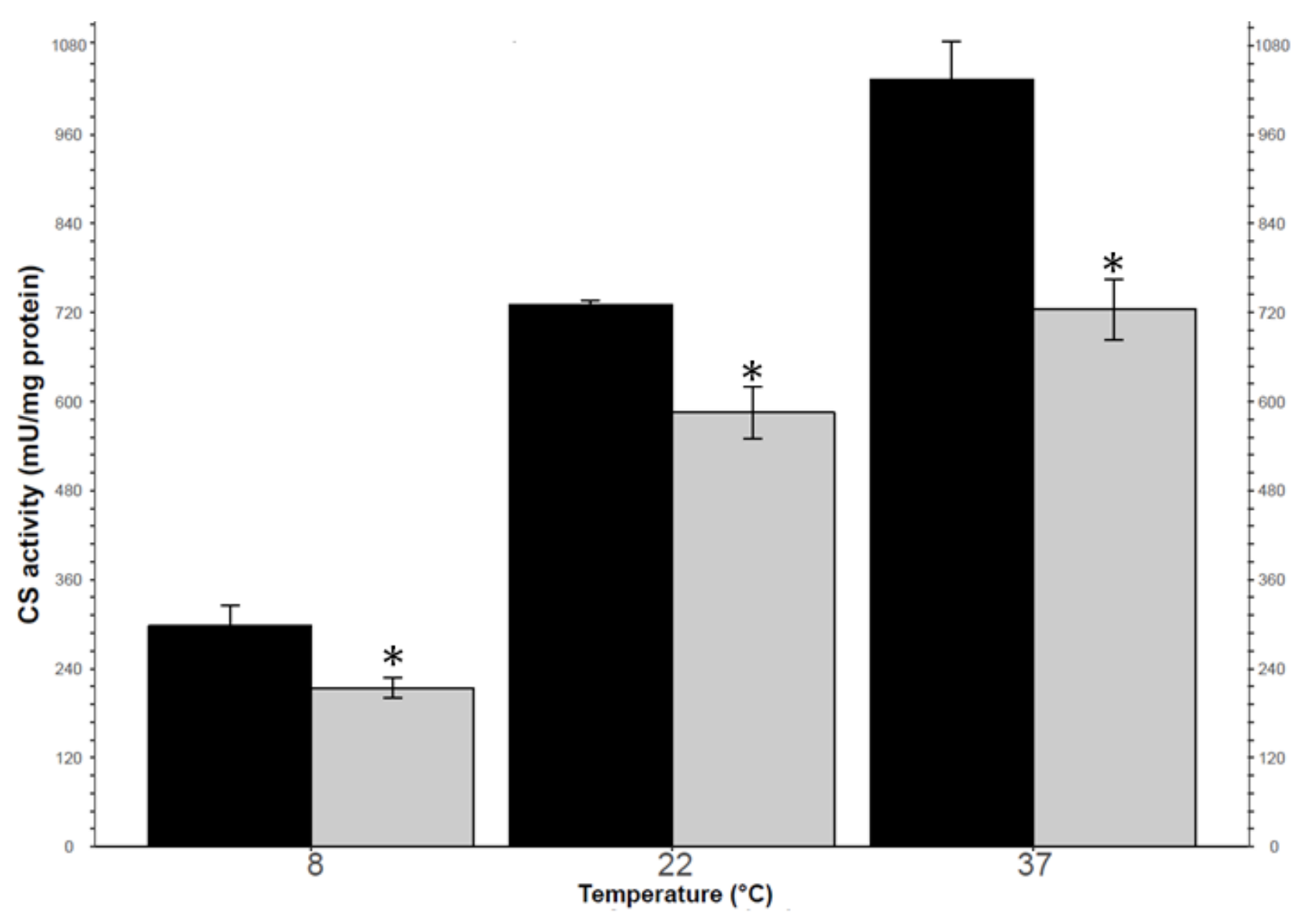

Euthermic $\square$ Hibernation

Fig. 3.2. Comparison of CS activity in whole tissue protein extracts expressed as mU activity per mg of total protein. ' $*$ ' indicates that the hibernating value is statistically different from the euthermic $(\mathrm{p}<0.05)$ by Student's t-test. Data represent a mean of 4 with error bars representing the standard error of the mean. 


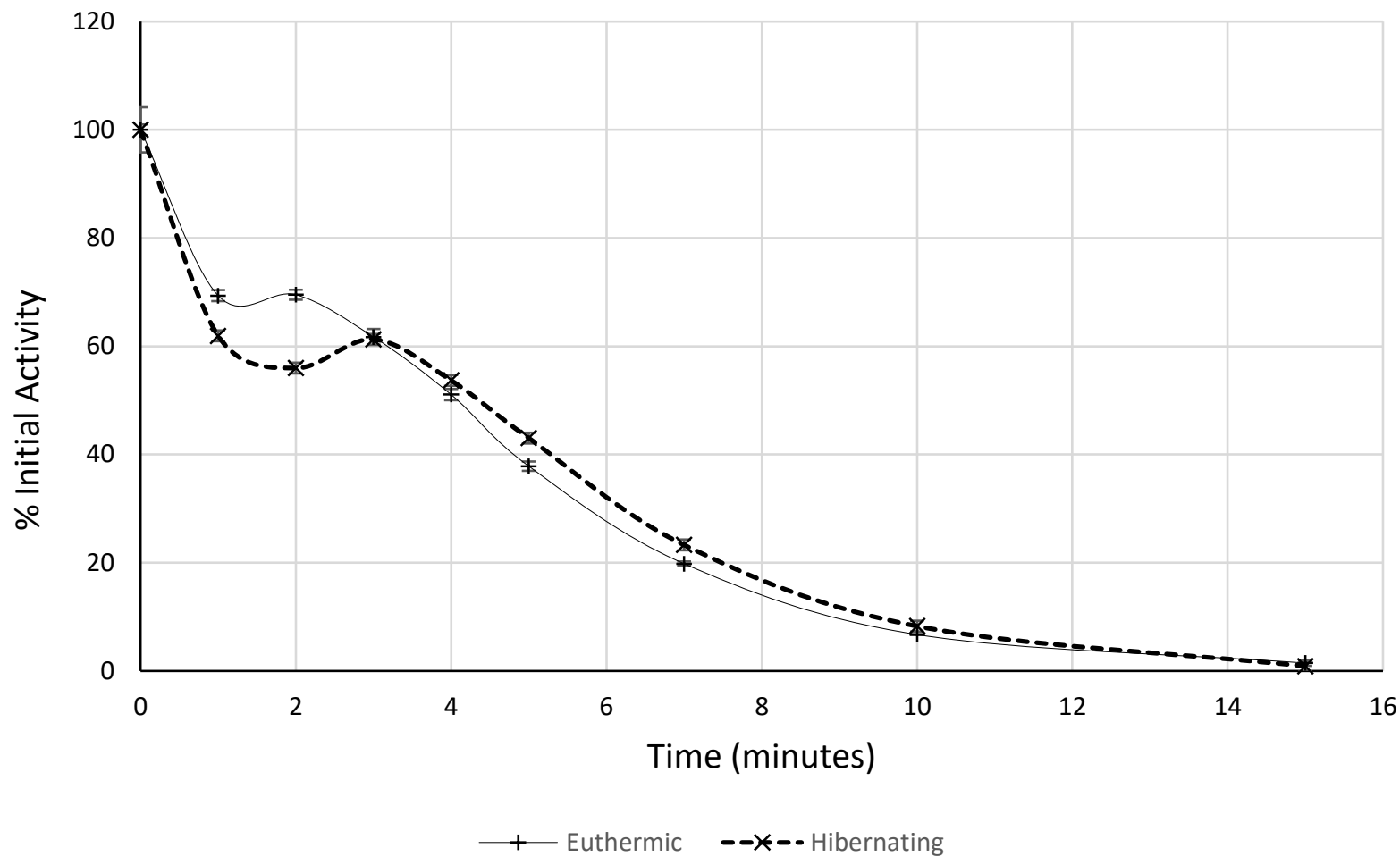

Fig. 3.3. Purified CS activity from Richardson's ground squirrel muscle tissue over time after varying incubation times at $60^{\circ} \mathrm{C}$. 


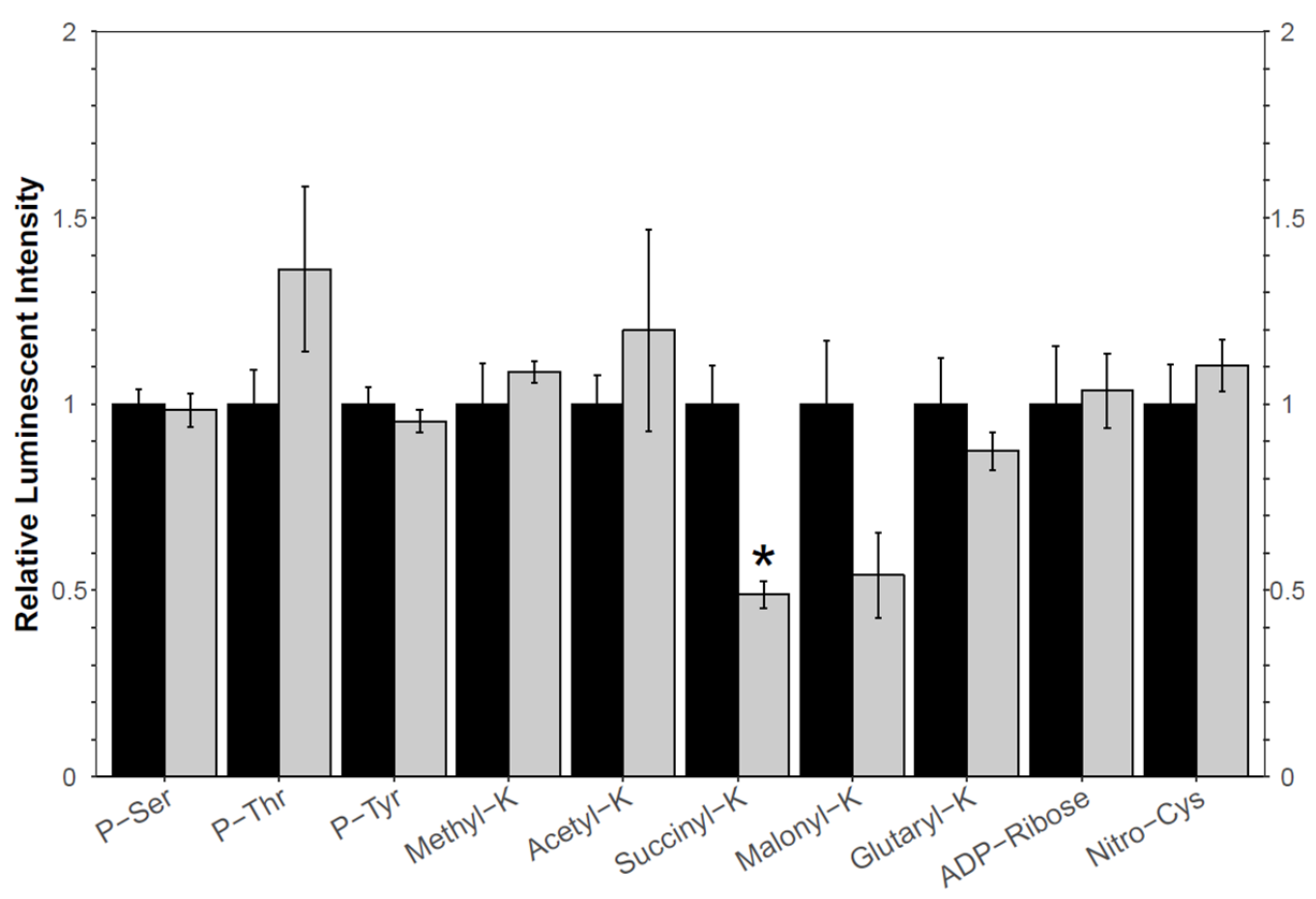

Euthermic $\square$ Hibernation

$\mathrm{ECL}$

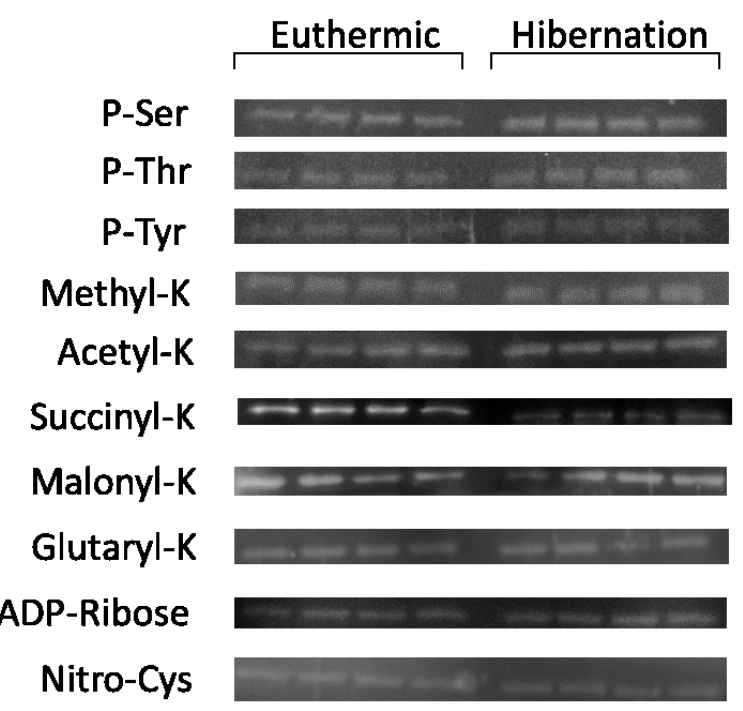

Coomassie
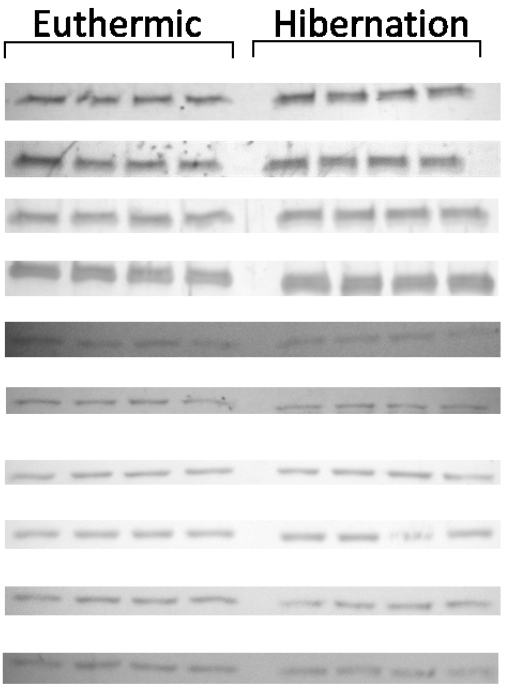

Fig. 3.4. Immunoblotting comparing the relative degree of various post-translational modifications on the purified citrate synthase from the control euthermic and hibernating Richardson's ground squirrels (Urocitellus richardsonii). Data represent the mean with standard error of the mean given in the error bars. ' $*$ ' denotes a hibernating parameter that is significantly different from the corresponding control euthermic by a two-tailed Student's t-test, $\mathrm{p}<0.05$. 


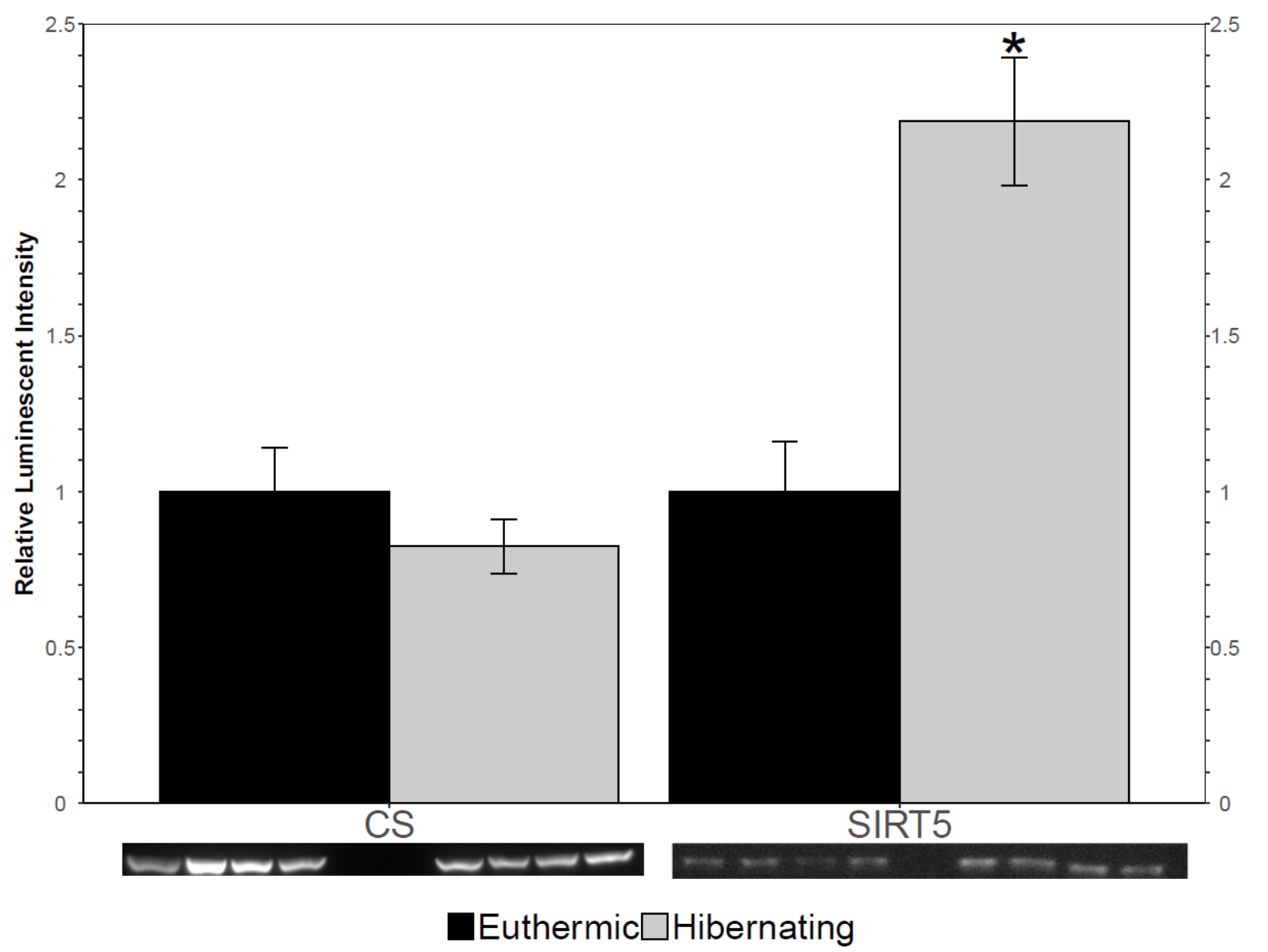

Fig. 3.5. Immunoblot comparing the relative level of CS and SIRT5 in euthermic and hibernating Richardson's ground squirrel (Urocitellus richardsonii) muscle extracts. '*' denotes that the hibernating parameter is statistically different from the euthermic control by a 2-tailed Student's t-test $(\mathrm{p}<0.05)$. 

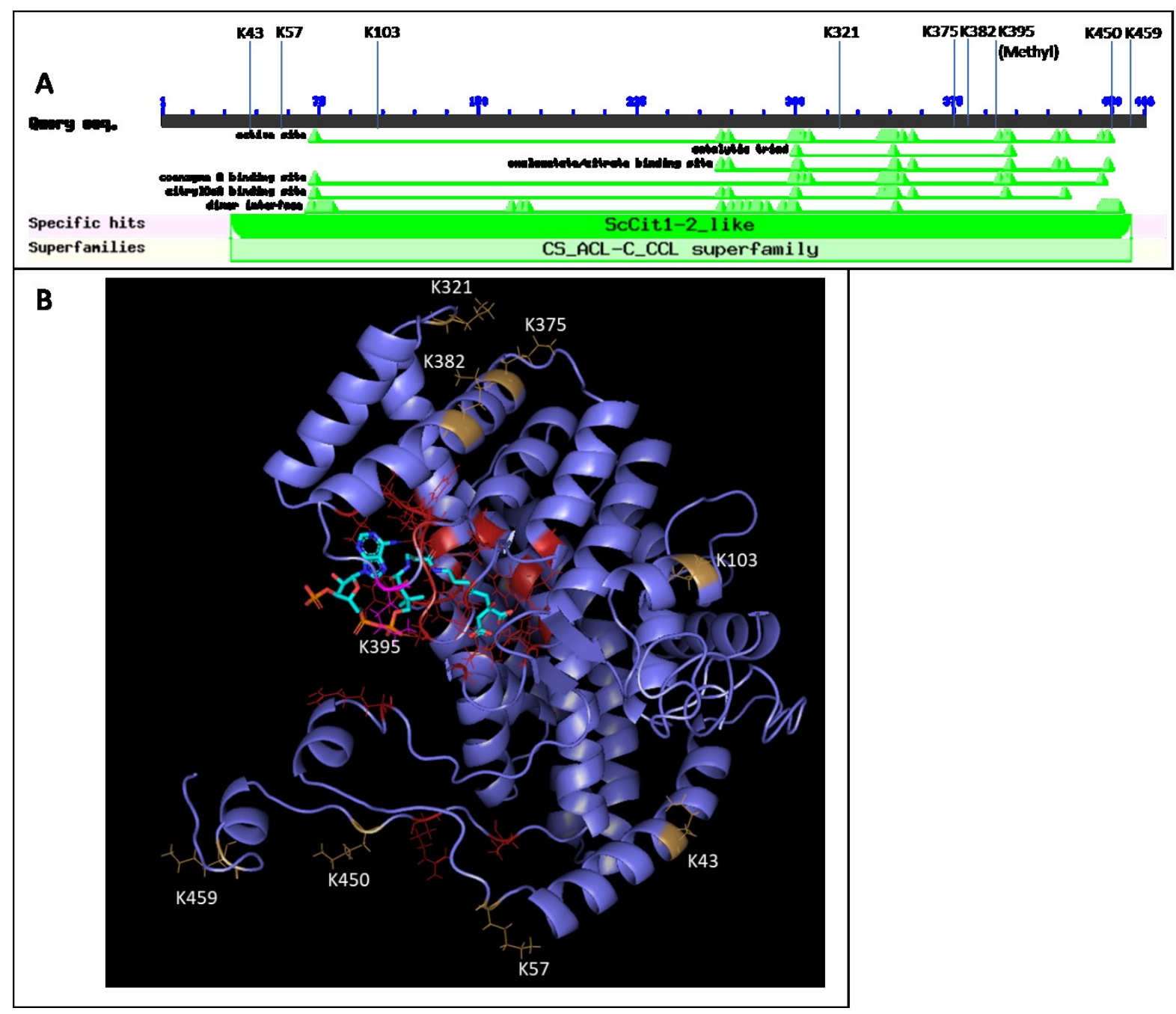

Fig. 3.6. A. Map of the known lysine succinylation sites (and the regulatory K395 methylation site) from the human homolog over the amino acids corresponding to the annotated conserved functional domains of the 13-lined ground squirrel citrate synthase (CS). B. Model showing the predicted structure of CS in Ictidomys tridecemlineatus with an analog of the substrates (Scitryldethia coenzyme A) within the active site. Residues associated with the active site (according to NCBI's conserved domains) are show in red. Lysine residues for which there exists evidence for succinylation are shown in than tan colour and labeled according to position in the sequence. The lysine methylation site known to be important in regulation of the enzyme is shown in magenta (K395). 


\section{Chapter 3 References}

Blinova, K., Carroll, S., Bose, S., Smirnov, A. V., Harvey, J. J., Knutson, J. R., \& Balaban, R. S. (2005). Distribution of mitochondrial NADH fluorescence lifetimes: Steady-state kinetics of matrix NADH interactions. Biochemistry, 44(7), 2585-2594. https://doi.org/10.1021/bi0485124

Bradford, M. M. (1976). A rapid and sensitive method for the quantitation of microgram quantities of protein utilizing the principle of protein-dye binding. Analytical Biochemistry, 72(1-2), 248-254. https://doi.org/10.1016/0003-2697(76)90527-3

Brooks, S. P. (1994). A program for analyzing enzyme rate data obtained from a microplate reader. Biotechniques, 17(6), 1154-1161. Retrieved from http://www.ncbi.nlm.nih.gov/entrez/query.fcgi?cmd=Retrieve $\& d b=$ PubMed\&dopt=Citation \&list_uids $=7873186$

Brooks, S. P. J. (1992). A simple computer program with statistical tests for the analysis of enzyme kinetics. BioTechniques, 13(6), 906-911. Retrieved from http://www.ncbi.nlm.nih.gov/pubmed/1476744

Bulutoglu, B., Garcia, K. E., Wu, F., Minteer, S. D., \& Banta, S. (2016). Direct evidence for metabolon formation and substrate channeling in recombinant TCA cycle enzymes. $A C S$ Chemical Biology, acschembio.6b00523. https://doi.org/10.1021/acschembio.6b00523

Chazarin, B., Storey, K. B., Ziemianin, A., Chanon, S., Plumel, M., Chery, I., ... Bertile, F. (2019). Metabolic reprogramming involving glycolysis in the hibernating brown bear skeletal muscle. Frontiers in Zoology, 16(1), 12. https://doi.org/10.1186/s12983-019-0312-2

Chinopoulos, C. (2013). Which way does the citric acid cycle turn during hypoxia? The critical role of $\alpha$-ketoglutarate dehydrogenase complex. Journal of Neuroscience Research, 91(8), 1030-1043. https://doi.org/10.1002/jnr.23196

Choudhary, C., Kumar, C., Gnad, F., Nielsen, M. L., Rehman, M., Walther, T. C., ... Mann, M. (2009). Lysine acetylation targets protein complexes and co-regulates major cellular functions. Science, 325(5942), 834-840. https://doi.org/10.1126/science.1175371

Eaton, S. L., Roche, S. L., Llavero Hurtado, M., Oldknow, K. J., Farquharson, C., Gillingwater, T. H., \& Wishart, T. M. (2013). Total protein analysis as a reliable loading control for quantitative fluorescent western blotting. PLoS ONE, 8(8). https://doi.org/10.1371/journal.pone.0072457

Geiser, F. (1988). Reduction of metabolism during hibernation and daily torpor in mammals and birds: temperature effect or physiological inhibition? Journal of Comparative Physiology B, 158(1), 25-37. https://doi.org/10.1007/BF00692726

Gibson, G. E., Xu, H., Chen, H. L., Chen, W., Denton, T. T., \& Zhang, S. (2015). Alphaketoglutarate dehydrogenase complex-dependent succinylation of proteins in neurons and 
neuronal cell lines. Journal of Neurochemistry, 134(1), 86-96.

https://doi.org/10.1111/jnc.13096

Green, S. R., \& Storey, K. B. (2020). Regulation of the $\alpha$-ketoglutarate dehydrogenase complex during hibernation in a small mammal, the Richardson's ground squirrel (Urocitellus richardsonii). Biochimica et Biophysica Acta - Proteins and Proteomics, 1868(9). https://doi.org/10.1016/j.bbapap.2020.140448

Huang, Y. ming M., Huber, G. A., Wang, N., Minteer, S. D., \& McCammon, J. A. (2018). Brownian dynamic study of an enzyme metabolon in the TCA cycle: Substrate kinetics and channeling. Protein Science, 27(2), 463-471. https://doi.org/10.1002/pro.3338

Kim, M. H., Park, K., Gwag, B. J., Jung, N. P., Oh, Y. K., Shin, H. C., \& Choi, I. H. (2000). Seasonal biochemical plasticity of a flight muscle in a bat, Murina leucogaster. Comparative Biochemistry and Physiology - A Molecular and Integrative Physiology, 126(2), 245-250. https://doi.org/10.1016/S1095-6433(00)00203-8

Klug, B. J., \& Brigham, R. M. (2015). Changes to metabolism and cell physiology that enable mammalian hibernation. Springer Science Reviews, 3(1), 39-56. https://doi.org/10.1007/s40362-015-0030-x

Kozlowski, L. P. (2017). Proteome-pI: Proteome isoelectric point database. Nucleic Acids Research, 45(D1), D1112-D1116. https://doi.org/10.1093/nar/gkw978

Landau, B. R., \& Dawe, A. R. (1958). Respiration in the hibernation of the 13-lined ground squirrel. American Journal of Physiology-Legacy Content, 194(1), 75-82. https://doi.org/10.1152/ajplegacy.1958.194.1.75

Larsen, S., Nielsen, J., Hansen, C. N., Nielsen, L. B., Wibrand, F., Stride, N., ... Hey-Mogensen, M. (2012). Biomarkers of mitochondrial content in skeletal muscle of healthy young human subjects. Journal of Physiology, 590(14), 3349-3360. https://doi.org/10.1113/jphysiol.2012.230185

Li, X., Wu, F., Qi, F., \& Beard, D. A. (2011). A database of thermodynamic properties of the reactions of glycolysis, the tricarboxylic acid cycle, and the pentose phosphate pathway. Database, 2011. https://doi.org/10.1093/database/bar005

Lombard, D. B., Tishkoff, D. X., \& Bao, J. (2011). Mitochondrial sirtuins in the regulation of mitochondrial activity and metabolic adaptation. Handbook of Experimental Pharmacology, 206, 163-188. https://doi.org/10.1007/978-3-642-21631-2_8

Małecki, J. drzej, Jakobsson, M. E., Ho, A. Y. Y., Moen, A., Rustan, A. C., \& Falnes, P. (2017). Uncovering human METTL12 as a mitochondrial methyltransferase that modulates citrate synthase activity through metabolite-sensitive lysine methylation. Journal of Biological Chemistry, 292(43), 17950-17962. https://doi.org/10.1074/jbc.M117.808451 
Marchler-Bauer, A., Bo, Y., Han, L., He, J., Lanczycki, C. J., Lu, S., ... Bryant, S. H. (2017). CDD/SPARCLE: Functional classification of proteins via subfamily domain architectures. Nucleic Acids Research, 45(D1), D200-D203. https://doi.org/10.1093/nar/gkw1129

Marchler-Bauer, A., Derbyshire, M. K., Gonzales, N. R., Lu, S., Chitsaz, F., Geer, L. Y., ... Bryant, S. H. (2015). CDD: NCBI's conserved domain database. Nucleic Acids Research, 43(D1), D222-D226. https://doi.org/10.1093/nar/gku1221

Mathers, K. E., \& Staples, J. F. (2019). Differential posttranslational modification of mitochondrial enzymes corresponds with metabolic suppression during hibernation. American Journal of Physiology-Regulatory, Integrative and Comparative Physiology, 317(2), R262-R269. https://doi.org/10.1152/ajpregu.00052.2019

Mertins, P., Qiao, J. W., Patel, J., Udeshi, N. D., Clauser, K. R., Mani, D. R., ... Carr, S. A. (2013). Integrated proteomic analysis of post-translational modifications by serial enrichment. Nature Methods, 10(7), 634-637. https://doi.org/10.1038/nmeth.2518

Mukherjee, A., \& Srere, P. A. (1976). Purification of and mechanism studies on citrate Synthase. Use of biospecific adsorption elution techniques. Journal of Biological Chemistry, 251(5), 1476-1480. https://doi.org/10.1016/s0021-9258(17)33764-x

Nazaret, C., Heiske, M., Thurley, K., \& Mazat, J. P. (2009). Mitochondrial energetic metabolism: A simplified model of TCA cycle with ATP production. Journal of Theoretical Biology, 258(3), 455-464. https://doi.org/10.1016/j.jtbi.2008.09.037

Park, J., Chen, Y., Tishkoff, D. X., Peng, C., Tan, M., Dai, L., ... Zhao, Y. (2013). SIRT5Mediated Lysine Desuccinylation Impacts Diverse Metabolic Pathways. Molecular Cell, 50(6), 919-930. https://doi.org/10.1016/j.molcel.2013.06.001

Rardin, M. J., He, W., Nishida, Y., Newman, J. C., Carrico, C., Danielson, S. R., ... Verdin, E. (2013). SIRT5 regulates the mitochondrial lysine succinylome and metabolic networks. Cell Metabolism, 18(6), 920-933. https://doi.org/10.1016/j.cmet.2013.11.013

Rhein, V. F., Carroll, J., Ding, S., Fearnley, I. M., \& Walker, J. E. (2017). Human METTL12 is a mitochondrial methyltransferase that modifies citrate synthase. FEBS Letters, 591(12), 1641-1652. https://doi.org/10.1002/1873-3468.12649

Rouble, A. N., \& Storey, K. B. (2015). Characterization of the SIRT family of NAD+-dependent protein deacetylases in the context of a mammalian model of hibernation, the thirteen-lined ground squirrel. Cryobiology, 71(2), 334-343. https://doi.org/10.1016/j.cryobiol.2015.08.009

Roy, A., Kucukural, A., \& Zhang, Y. (2010). I-TASSER: A unified platform for automated protein structure and function prediction. Nature Protocols, 5(4), 725-738. https://doi.org/10.1038/nprot.2010.5 
Siess, E. A., Kientsch-Engel, R. I., \& Wieland, O. H. (1984). Concentration of free oxaloacetate in the mitochondrial compartment of isolated liver cells. The Biochemical Journal, 218(1), 171-176. https://doi.org/10.1042/bj2180171

Sievers, F., Wilm, A., Dineen, D., Gibson, T. J., Karplus, K., Li, W., ... Higgins, D. G. (2011). Fast, scalable generation of high-quality protein multiple sequence alignments using Clustal Omega. Molecular Systems Biology, 7, 539. https://doi.org/10.1038/msb.2011.75

Srere, P. A., \& Matsuoka, Y. (1972). Inhibition of rat citrate synthase by acetoacetyl CoA and NADH. Biochemical Medicine. https://doi.org/10.1016/0006-2944(72)90047-6

Staples, J. F. (2014). Metabolic suppression in mammalian hibernation: The role of mitochondria. Journal of Experimental Biology, 217(12), 2032-2036. https://doi.org/10.1242/jeb.092973

St-Pierre, J., \& Boutilier, R. G. (2001). Aerobic capacity of frog skeletal muscle during hibernation. Physiological and Biochemical Zoology, 74(3), 390-397. https://doi.org/10.1086/320428

Stuart, J. A., Ooi, E. L., \& Ballantyne, J. S. (1998). Maximal activities of enzymes of intermediary metabolism in the estivating terrestrial snail Cepaea nemoralis. Comparative Biochemistry and Physiology - B Biochemistry and Molecular Biology, 120(2), 417-423. https://doi.org/10.1016/S0305-0491(98)10027-5

Subramanian, S., \& Ross, P. D. (1984). Dye-ligand affinity chromatography: The interaction of Cibacron blue F3GA ${ }^{\circledR}$ with proteins and enzyme. Critical Reviews in Biochemistry and Molecular Biology, 16(2), 169-205. https://doi.org/10.3109/10409238409102302

Tompa, P., Batke, J., Ovadi, J., Welch, G. R., \& Srere, P. A. (1987). Quantitation of the interaction between citrate synthase and malate dehydrogenase. Journal of Biological Chemistry, 262(13), 6089-6092. https://doi.org/10.1074/jbc.273.45.29540

Van Breukelen, F., \& Martin, S. L. (2015). The hibernation continuum: Physiological and molecular aspects of metabolic plasticity in mammals. Physiology, 30(4), 273-281. https://doi.org/10.1002/aja.1000900202

Venkat, S., Chen, H., McGuire, P., Stahman, A., Gan, Q., \& Fan, C. (2019). Characterizing lysine acetylation of Escherichia coli type II citrate synthase. FEBS Journal, 286(14), 2799-2808. https://doi.org/10.1111/febs.14845

Vigelsø, A., Andersen, N. B., \& Dela, F. (2014). The relationship between skeletal muscle mitochondrial citrate synthase activity and whole body oxygen uptake adaptations in response to exercise training. International Journal of Physiology, Pathophysiology and Pharmacology, 6(2), 84-101.

Wang, L. C. H. (1979). Time patterns and metabolic rates of natural torpor in the Richardson's ground squirrel. Canadian Journal of Zoology, 57(2), 149-155. https://doi.org/10.1139/z79012 
Wang, L. C. H., \& Lee, T. F. (2011). Torpor and Hibernation in Mammals: Metabolic, Physiological, and Biochemical Adaptations. In Comprehensive Physiology (pp. 507-532). Hoboken, NJ, USA: John Wiley \& Sons, Inc. https://doi.org/10.1002/cphy.cp040122

Weinert, B. T., Schölz, C., Wagner, S. A., Iesmantavicius, V., Su, D., Daniel, J. A., \& Choudhary, C. (2013). Lysine succinylation is a frequently occurring modification in prokaryotes and eukaryotes and extensively overlaps with acetylation. Cell Reports, 4(4), 842-851. https://doi.org/10.1016/j.celrep.2013.07.024

Wickler, S. J., Horwitz, B. A., \& S.Kott, K. (1987). Muscle function in hibernating hamsters: A natural analog to bed rest? Journal of Thermal Biology, 12(2), 163-166. https://doi.org/10.1016/0306-4565(87)90058-1

Yang, J., Yan, R., Roy, A., Xu, D., Poisson, J., \& Zhang, Y. (2015). The I-TASSER Suite: protein structure and function prediction. Nature Methods, 12(1), 7-8. https://doi.org/10.1038/nmeth.3213

Zhang, J., \& Storey, K. B. (2016). RBioplot: an easy-to-use R pipeline for automated statistical analysis and data visualization in molecular biology and biochemistry. PeerJ, 4, e2436. https://doi.org/10.7717/peerj.2436

Zhang, Y. (2008). I-TASSER server for protein 3D structure prediction. BMC Bioinformatics, 9(1), 40. https://doi.org/10.1186/1471-2105-9-40

Zhang, Z., Tan, M., Xie, Z., Dai, L., Chen, Y., \& Zhao, Y. (2011). Identification of lysine succinylation as a new post-translational modification. Nature Chemical Biology, 7(1), 5863. https://doi.org/10.1038/nchembio.495

Zhao, S., Xu, W., Jiang, W., Yu, W., Lin, Y., Zhang, T., ... Guan, K. L. (2010). Regulation of cellular metabolism by protein lysine acetylation. Science, 327(5968), 1000-1004. https://doi.org/10.1126/science.1179689 


\section{Chapter 4: Regulation of Muscle KGDC in Hibernation}




\title{
Regulation of the $\alpha$-ketoglutarate dehydrogenase complex during hibernation in a small mammal, the Richardson's ground squirrel (Urocitellus richardsonii)
}

\author{
Stuart R. Green and Kenneth B. Storey
}

Institute of Biochemistry, Carleton University, 1125 Colonel By Drive, Ottawa ON, K1S 5B6, Canada

The following study has been published in the Journal of Thermal Biology

Green, S.R. and Storey, K.B. (2020) Regulation of the $\alpha$-ketoglutarate dehydrogenase complex during hibernation in a small mammal, the Richardson's ground squirrel (Urocitellus richardsonii). Biochimica et Biophysica Acta (BBA) - Proteins and Proteomics . 1868 (9). DOI: 10.1016/j.bbapap.2020.140448 


\title{
Highlights
}

- KGDC is regulated in hibernation in the Richardson's ground squirrel muscle.

- Distinct post translational status is associated with the hibernating form.

- Hibernation associated with decreased affinity for coenzyme A.

\begin{abstract}
The citric acid cycle (CAC) is a central metabolic pathway that links carbohydrate, lipid, and amino acid metabolism in the mitochondria and, hence, is a crucial target for metabolic regulation. The $\alpha$-ketoglutarate dehydrogenase complex (KGDC) is the rate-limiting step of the $\mathrm{CAC}$, the three enzymes of the complex catalyzing the transformation of $\alpha$-ketoglutarate to succinyl-CoA with the release of $\mathrm{CO}_{2}$ and reduction of NAD to NADH. During hibernation, the metabolic rate of small mammals is suppressed, in part due to reduced body temperature but also active controls that suppress aerobic metabolism. The present study examined KGDC regulation during hibernation in skeletal muscle of the Richardson's ground squirrel (Urocitellus richardsonii). The KGDC was partially purified from skeletal muscle of euthermic and hibernating ground squirrels and kinetic properties were evaluated at $5^{\circ}, 22^{\circ}$, and $37^{\circ} \mathrm{C}$. $\mathrm{KGDC}$ from hibernator muscle at all temperatures compared with euthermic controls exhibited a decreased affinity for $\mathrm{CoA}$ as well as reduced activation by $\mathrm{Ca}^{2+}$ ions at $5^{\circ} \mathrm{C}$ from both euthermic and hibernating conditions. Co-immunoprecipitation was employed to isolate the E1, E2 and E3 enzymes of the complex (OGDH, DLST, DLD) to allow immunoblot analysis of posttranslational modifications (PTMs) of each enzyme. The results showed elevated phosphotyrosine content on all three enzymes during hibernation as well as increased ADP-ribosylation and succinylation of hibernator OGDH. Taken together these results show that the KGDC is
\end{abstract}


regulated by posttranslational modifications and temperature effects to reorganize enzyme activity and mitochondrial function to aid suppression of mitochondrial activity during hibernation.

Keywords: citric acid cycle, hibernation, muscle, Richardson's ground squirrel, posttranslational modifications, ketoglutarate dehydrogenase complex

\author{
Abbreviations \\ CAC - Citric Acid Cycle \\ DLD - dihydrolipoamide dehydrogenase (E3 subunit of KGDC) \\ DLST - dihydrolipoamide succinyl transferase (E2 subunit of KGDC) \\ KGDC - ketoglutarate dehydrogenase complex \\ $K_{A}$ - Activator concentration yielding half-maximal enzyme activation \\ $K_{m}$ - Michaelis constant (substrate concentration yielding half-maximal enzyme activity) \\ OGDH - oxoglutarate dehydrogenase (E1 subunit of KGDC) \\ PEG - Polyethylene glycol \\ PTM - post-translational modification \\ $\alpha-K G$ - alpha-ketoglutarate
}




\subsection{Introduction}

Mammalian hibernation is a complex phenomenon that is controlled at a molecular level in order to reduce metabolic rate and allow animals to conserve energy and metabolic fuels during the winter months. The reduction in metabolic rate is mediated by physiological and biochemical changes including a profound decrease in core body temperature, often to near $0{ }^{\circ} \mathrm{C}$ and alterations to enzymes involved in central metabolic pathways. A key metabolic strategy in hibernating mammals is the preferential consumption of extensive fat reserves built up during the summer feeding and accordingly numerous studies have sought to evaluate the enzymatic mechanisms that suppresses glycolytic activity (carbohydrate fuel use) during hibernation. Examples of such studies in various ground squirrel species have identified regulation of glycogen phosphorylase and pyruvate dehydrogenase in numerous tissues (Brooks \& Storey, 1992), control of hexokinase in the skeletal muscle (Abnous \& Storey, 2008), and of phosphofructokinase in muscle (MacDonald \& Storey, 2005) as all contributing to the suppression of glycolysis during hibernation. However, less is understood in regards to the regulation of other pathways responsible for generating cellular ATP during hibernation. The citric acid cycle (CAC) is the central metabolic hub for aerobic respiration that integrates carbon sources from glycolysis, fatty acid catabolism, and amino acid breakdown and therefore likely constitutes an important regulatory target during hibernation.

The Richardson's ground squirrel (Urocitellus richardsonii) is a small obligate hibernator that undergoes prolonged periods of hibernation from mid-July to mid-March, allowing for an $88 \%$ savings of metabolic energy over this time (Wang, 1979). During bouts of torpor the core body temperature of the animal cools to near ambient with a minimum temperature of around 2-3 ${ }^{\circ} \mathrm{C}$ (Wang \& Lee, 2011). While part of the reduction in metabolic rate during hibernation can be 
attributed to the lowered body temperature, determinations of $\mathrm{Q}_{10}$ values have demonstrated that the reduction in metabolic rate is greatest between $20-30{ }^{\circ} \mathrm{C}\left(\mathrm{Q}_{10}=4.1\right)$ and decreases at lower body temperatures (between $0.1-10{ }^{\circ} \mathrm{C} \mathrm{Q}_{10}=2.85$ ) as measured by $\mathrm{O}_{2}$ consumption (Geiser, 1988). This indicates that another, active mechanism exists to reduce metabolic rate during the entrance of torpor. Owing to its importance as a central metabolic hub necessary for producing the reducing equivalents needed to generate ATP in the mitochondria, the CAC is a likely target for metabolic regulation during hibernation.

The $\alpha$-ketoglutarate dehydrogenase complex (KGDC) (E.C. 1.2.4.2), is responsible for catalyzing a complicated rate-limiting reaction in the CAC that removes a carboxyl group from $\alpha$-ketoglutarate (releasing $\mathrm{CO}_{2}$ ), and transfers the remaining succinyl moiety to coenzyme A (CoA) while reducing NAD to NADH (Shi et al., 2011):

$$
\alpha-\text { ketoglutarate }+\mathrm{NAD}^{+}+\mathrm{CoA} \rightarrow \text { Succinyl } \mathrm{CoA}+\mathrm{CO}_{2}+\mathrm{NADH} .
$$

Studies in rat heart have demonstrated that the rate of flux through the CAC correlated with the activity of KGDC, suggesting the importance of this reaction in regulating metabolic rate (Cooney, Taegtmeyer, \& Newsholme, 1981). In addition to being the rate-limiting step of the $\mathrm{CAC}$, the reaction catalyzed by KGDC is also one of only two essentially irreversible reactions within the $\mathrm{CAC}$ (the other being that catalyzed by citrate synthase), meaning that control of this step is crucial to the flux of substrates through the CAC (Chinopoulos, 2013). Since the KGDC is localized within the mitochondrial matrix, the NADH produced at this step (and other steps within the CAC) can be used directly by the electron transport chain (ETC) in the establishment of the chemiosmotic gradient necessary for the production of ATP. The KGDC is structurally homologous to two other oxo-acid dehydrogenase complexes; the pyruvate 
dehydrogenase complex (PDC) and the branched chain keto-acid dehydrogenase complex (BCKDC). In eukaryotic organisms this family of enzyme complexes are made up of a core of 60 E2 (dihydrolipoyl succinyl-transferase) subunits arranged as a dodecahedral structure surrounded by 6 non-covalently interacting E1 (oxoglutarate dehydrogenase) dimers and E3 (dihydrolipoamide dehydrogenase) proteins (Denton, 2009; Izard et al., 1999). Owing to the presence of multiple copies, these complexes commonly have molecular weights in excess of 5 MDa (Reed, 1974). The first enzyme of the KGDC, E1 or OGDH, uses a thiamine diphosphate (TPP) cofactor that generates a transient carbanion intermediate to catalyze a nucleophilic attack on the ketone carbon to release $\mathrm{CO}_{2}$. This leaves a succinyl group that is transferred to an oxidized lipoamide prosthetic cofactor on the second enzyme of the complex, dihydrolipoamide succinyl transferase (DLST) that then transfers the succinyl group to CoA to form succinyl-CoA, the final product. All keto-acid dehydrogenases share a common third enzyme in their reactions whose role is to oxidize the reduced dihydrolipoamide prosthetic group of the E2 enzyme to return it to the active disulfide form using a prosthetic FAD molecule and NAD to reset the FAD cofactor. Both PDC and BCKDC have homologues for the first two enzymes of the complex that use the same cofactors and carry out similar reactions, but with different keto-acid substrates.

The current study seeks to ascertain how KGDC function is affected during winter hibernation compared to euthermic conditions. This was accomplished by measuring the affinity of a partially purified complex for its substrates at physiologically relevant temperatures encountered by the animal during the hibernation season. Furthermore, differences in the relative degree of various post-translational modifications were assessed using an immunoblotting method to give determine how this complex is structurally altered during hibernation. 


\subsection{Methods}

\subsubsection{Animals}

Animal treatment protocols for control euthermic and hibernating Richardson's ground squirrel were performed as previously described (MacDonald \& Storey, 1998). All animals were euthanized by decapitation and were quickly dissected with the muscle tissues frozen in liquid nitrogen and subsequently shipped on dry ice to Carleton University from the University of Calgary where they were stored at $-80^{\circ} \mathrm{C}$ until use. Animal care and experimentation had the prior approval of the university animal care committee and abided by the Canadian Council on Animal Care guidelines.

\subsubsection{KGDC Enrichment}

KGDC was enriched from preparations of Richardson's ground squirrel skeletal muscle. Samples of frozen muscle were homogenized 1:5 w/v in ice-cold homogenate buffer $(25 \mathrm{mM}$ Tris, pH 7.2, 0.2 mM EDTA, 0.2 mM EGTA, 15 mM $\beta$-glycerol phosphate, $5 \mathrm{mM} \beta$ mercaptoethanol, $10 \% \mathrm{v} / \mathrm{v}$ glycerol) with a few crystals of phenylmethylsulfonyl fluoride (PMSF) protease inhibitor added immediately before homogenization. After centrifugation for 30 min at $4{ }^{\circ} \mathrm{C}$ at $13,500 \times \mathrm{g}$ in an Eppendorf 5810R tabletop centrifuge, the supernatant was removed and saved while the pellet was discarded. An aliquot of a $50 \%$ solution of polyethylene glycol (PEG) 8000 was then added to the supernatant to a final concentration of $2 \% \mathrm{w} / \mathrm{v}$ to induce precipitation followed by centrifugation as before. Both the supernatant and a resuspended pellet were then tested for KGDC activity, with the supernatant found to contain all the activity. The PEG concentration of the supernatant was then increased to $6 \% \mathrm{w} / \mathrm{v}$, followed by centrifugation at $750 \times \mathrm{g}$ for $20 \mathrm{~min}$ in a VWR Clinical 50 centrifuge. The supernatant was removed and the pellet was resuspended in 0.25 volumes of homogenate buffer relative to the 
supernatant. After mixing the resuspended pellet, a third centrifugation at $13,500 \times \mathrm{g}$ for $30 \mathrm{~min}$ was used to pellet any proteins/residue that could not be solubilized back into the buffer. The supernatant from this step was then applied to a Sephacryl S-400 column measuring $20 \mathrm{~cm} \times 1$ $\mathrm{cm}$ pre-equilibrated in $100 \mathrm{~mL}$ of homogenate buffer. This was filtered through the column by flowing $40 \mathrm{~mL}$ of homogenate buffer through it. Forty-drop fractions (approximately $1.3 \mathrm{~mL}$ ) were collected from the S-400 column and each was assayed for KGDC activity. Fractions with the highest activity (5-6 fractions) were pooled for further analysis of KGDC kinetic parameters.

\subsubsection{KGDC Assay}

Assay of KGDC activity used a Thermo Scientific Multiskan Spectrum microplate reader measuring absorbance at $340 \mathrm{~nm}$ during production of NADH from $\mathrm{NAD}^{+}$with assay conditions based on those described previously (Gibson et al., 2015). All reagents were prepared in $50 \mathrm{mM}$ Tris buffer $\mathrm{pH}$ 7.8. Standard optimal reaction conditions contained $1.5 \mathrm{mM} \mathrm{CaCl}_{2}, 0.5 \mathrm{mM}$ $\mathrm{MgCl}_{2}, 0.25 \mathrm{mM}$ EDTA, $0.5 \mathrm{mM}$ dithiothreitol (DTT), $0.05 \% \mathrm{v} / \mathrm{v}$ Triton X-100, $0.150 \mathrm{mM}$ thiamine pyrophosphate (TPP), $1 \mathrm{mM} \mathrm{NAD}, 1 \mathrm{mM} \alpha$-ketoglutarate (KG), and $0.3325 \mathrm{mM}$ coenzyme-A (CoA). Reactions were initiated by the addition of $20 \mu \mathrm{L}$ of sample containing KGDC and were performed at $22{ }^{\circ} \mathrm{C}$ unless otherwise stated. $1 \mathrm{U}$ of KGDC activity was defined as the amount of KGDC activity needed to convert $1 \mu \mathrm{mol}$ of KG to succinyl-CoA per minute at the aforementioned substrate concentrations. For assays performed at $37^{\circ} \mathrm{C}$, the microplate with assay components was heated using the internal temperature controls of the Multiskan Spectrum instrument and temperature was confirmed as $37^{\circ} \mathrm{C}$ with a telethermometer prior to starting the reaction. A low-temperature VWR incubator was used to cool the microplate reader and microplates for assays requiring cooling to $5^{\circ} \mathrm{C}$ and a telethermometer was used to confirm a $5^{\circ}$ $\mathrm{C}$ temperature of the wells before beginning the reaction. 


\subsubsection{Co-immunoprecipitation}

To obtain a higher purity sample of the $\alpha-\mathrm{KGDH}$ complex suitable for use in testing for PTMs by immunoblotting analysis, a co-immunoprecipitation method was employed targeting the E1 enzyme of the complex, OGDH. Protein-A agarose beads (BioShop, \#PRA284) were used to bind an antibody recognizing OGDH. Beads were prepared by addition of rabbit OGDH antibody (GTX33374) to the beads, with $6 \mu 1$ of antibody added to $30 \mu 1$ of beads. To reduce contamination by the antibody in the final co-immunoprecipitation preparation, a cross-linking step was used to covalently attach the antibody to the beads. Beads were extracted from the stock and then briefly centrifuged. The supernatant was removed and replaced with phosphate buffer saline solution (PBS: $10 \mathrm{mM} \mathrm{Na}_{2} \mathrm{HPO}_{4}, 137 \mathrm{mM} \mathrm{NaCl}, 2.7 \mathrm{mM} \mathrm{KCl}, 2 \mathrm{mM} \mathrm{KH} 2 \mathrm{PO}_{4}, \mathrm{pH}$ 7.4). The beads and the PBS solution were mixed overnight at $4{ }^{\circ} \mathrm{C}$. The bead/PBS mixture was centrifuged briefly and the supernatant was decanted and replaced with an equal volume of dilution buffer (PBS solution with $1 \mathrm{mg} / \mathrm{mL}$ bovine serum albumin). After mixing for $10 \mathrm{~min}$, this solution was removed and $6 \mu \mathrm{l}$ of antibody diluted to $30 \mu \mathrm{l}$ in dilution buffer was mixed with the beads for $2 \mathrm{~h}$ at $4{ }^{\circ} \mathrm{C}$. Beads were then briefly centrifuged, the supernatant was discarded and an equal volume of dilution buffer was added and mixed with the beads for 5 min before being discarded after centrifugation and replaced with PBS buffer. An aliquot of dimethyl pimelimidate (DMP), prepared as $6.5 \mathrm{mg} / \mathrm{mL}$ in wash buffer $(0.2 \mathrm{M}$ triethanolamine in PBS buffer) and adjusted to $\mathrm{pH} 8-9$ was then added in a 1:1 ratio with the beads and mixed for 30 min at room temperature. The DMP solution was then discarded after brief centrifugation and then replaced with an equal volume of wash buffer and this was allowed to mix for $5 \mathrm{~min}$. The DMP and wash steps were repeated two more times to ensure adequate cross-linking between the bead and the antibody. After another 5 min wash, the supernatant was removed and replaced with an 
equal volume of quenching buffer ( $50 \mathrm{mM}$ ethanolamine in PBS) 3 times and incubated for 5 min each time. The quenching buffer was then removed after brief centrifugation and replaced with PBS and mixed for 5 min. Beads were then washed with $1 \mathrm{M}$ glycine $(\mathrm{pH} 3)$ in a 1:1 v/v ratio for $3 \times 10$ min to remove any unlinked antibody. Beads were then washed in wash buffer for $4 \times 5$ min each with mixing. Beads were stored in the final wash until use. This protocol was also used to prepare two negative controls, one that used non-specific mouse IgG on the beads, and the other that was not exposed to any antibody. SDS-PAGE analysis revealed one protein band that bound to both the of the negative controls indicating that its presence was not reflective of it being physically associated with the KGDC but rather that it non-specifically bound the bead itself. This band however was at a significantly different molecular weight from the proteins of interest and therefore did not interfere with the analysis of PTMs of the components of KGDC and was later identified as phosphofructokinase through mass spectroscopy.

Muscle samples were homogenized in the same manner as described before and subjected to the same PEG precipitation protocol to enrich for KGDC. Subsequently, samples were transferred into a low salt buffer $(10 \mathrm{mM}$ Tris $\mathrm{HCl}, 10 \mathrm{mM} \mathrm{NaCl}, 2 \mathrm{mM}$ EDTA, 0.1\% Triton X100, 1 mM PMSF, pH 7.5) by centrifuging $500 \mu \mathrm{L}$ aliquots through $5 \mathrm{~mL}$ of G-25 Sephadex equilibrated in the low salt buffer (Childers \& Storey, 2016) followed by diluting the sample to $0.5 \mathrm{mg} / \mathrm{ml}$ of protein. A $400 \mu \mathrm{l}$ aliquot of muscle sample was added for every $30 \mu \mathrm{L}$ of the antibody cross-linked beads. Samples were then incubated with the beads for $4 \mathrm{~h}$ at $4{ }^{\circ} \mathrm{C}$ with rotation. The supernatant was removed following brief low speed centrifugation and the beads were washed 4 times with $400 \mu$ of low salt buffer to remove any non-specifically bound proteins. Beads were then resuspended with $30 \mu \mathrm{L}$ of the low salt buffer (1:1). To these samples SDS-PAGE loading buffer (100 mM Tris buffer, $4 \% \mathrm{w} / \mathrm{v}$ SDS, $20 \% \mathrm{v} / \mathrm{v}$ glycerol, $0.2 \% \mathrm{w}: \mathrm{v}$ 
bromophenol blue, $10 \% \mathrm{v} / \mathrm{v} 2$-mercaptoethanol, $\mathrm{pH} 6.8$ ) was added (60 $\mu 1$ to a final volume of $120 \mu \mathrm{l})$ and samples were then boiled for $5 \mathrm{~min}$ to prepare them for gel electrophoresis.

\subsubsection{Mass Spectrometry}

Mass spectrometry was used to verify the identities of protein bands that were precipitated by the co-immunoprecipitation procedure. Bands were carefully excised from a $10 \%$ polyacrylamide gel following electrophoresis and Coomassie Brilliant Blue R-250 staining (see Western Blots) using a sterile razor blade and forceps. These gel slices were placed into individually labeled tubes and rinsed with destain solution to remove excess Coomassie stain. Samples were shipped to the proteomics center at Laval University where they were trypsindigested and then analyzed by tandem MS/MS spectrometry to identify the proteins in each band. MASCOT software was used as a search engine to query protein databases to identify the proteins. MS Data was viewed using Scaffold 4 proteomics software (Searle, 2010).

\subsubsection{Western Blots}

Western blots were performed to identify the individual protein components of KGDC following partial purification via the PEG precipitation and co-IP methods. Protein samples were first prepared by boiling 1:1 v/v with SDS-PAGE loading buffer (100 mM Tris, 4\% w/v SDS, $20 \% \mathrm{v} / \mathrm{v}$ glycerol, $0.2 \% \mathrm{w} / \mathrm{v}$ bromophenol blue, $\mathrm{pH} 6.8$ ) for $5 \mathrm{~min}$. Samples were then loaded onto a $10 \%$ SDS-PAGE gel and run for $60 \mathrm{~min}$ at $180 \mathrm{~V}$ in running buffer $(25 \mathrm{mM}$ Tris-base, $250 \mathrm{mM}$ glycine, $0.1 \% \mathrm{w} / \mathrm{v}$ SDS, $\mathrm{pH}$ 8.3-8.5). Proteins were then transferred to PVDF membrane using $240 \mathrm{~mA}$ of current for $90 \mathrm{~min}$ while immersed in transfer buffer ( $25 \mathrm{mM}$ Tris-base, 192 $\mathrm{mM}$ glycine, $20 \% \mathrm{v} / \mathrm{v}$ methanol, $\mathrm{pH} 8.8$ ). Membranes were then washed in TBST (20 mM Tris base, $140 \mathrm{mM} \mathrm{NaCl}, 0.05 \% \mathrm{v} / \mathrm{v}$ Tween-20, $\mathrm{pH}$ 7.6) for $5 \mathrm{~min}$ before being incubated overnight 
in primary antibody diluted 1:1000 v/v. Antibodies used for identification of the individual protein components were as follows:

1. Polyclonal anti-OGDH antibody (E1) (GeneTex, GTX33374)

2. Polyclonal anti-DLST antibody (E2) (GeneTex, GTX112555)

3. Polyclonal anti-DLD antibody (E3) (GeneTex, GTX101245)

In addition to these antibodies, other Western blots were performed to quantify the relative levels of various PTMs on the different components of KGDC that were separated on a gel following co-IP. These Western blots used pan specific antibodies that recognized various PTMs:

1. Anti-phosphorylated tyrosine (Invitrogen cat. \# 13-660)

2. Anti-phosphorylated serine (Invitrogen cat. \# 61-8100)

3. Anti-phosphorylated threonine (Invitrogen cat. \# 71-8200)

4. Anti-methylated lysine (StressMarq Biosciences Inc. cat. \# SPC-158F)

5. Anti-mono and di-methylated arginine (CovaLab cat. \# mab0002)

6. Anti-nitrosylated cysteine (Abcam cat. \# ab50185)

7. Anti-glutathione (Santa Cruz Biotechnology cat. \# sc-52399)

8. Anti-acetyl lysine (Santa Cruz Biotechnology cat. \# SC8663)

9. ADP ribose binding reagent conjugated to rabbit $\mathrm{Fc}$ of IgG (EMD Millipore cat \# MABE1016) 
10. Anti-succinyl lysine (PTM BioLabs cat \# PTM-401)

11. Anti-malonyl lysine (PTM BioLabs cat \# PTM-901)

12. Anti-glutaryl lysine (PTM BioLabs cat \# PTM-1151)

Following overnight incubation with the antibodies at $4{ }^{\circ} \mathrm{C}$, the membranes were washed for $3 \times 5$ min with TBST to remove unbound primary antibody. Membranes were then incubated for $30 \mathrm{~min}$ in an appropriate secondary antibody (either anti-mouse or anti-rabbit) conjugated to horseradish peroxidase and diluted 1:8000 v/v. Unbound secondary antibody was then washed off the membrane with $3 \times 5$ min washes of TBST. Visualization of the membranes was then performed using an enhanced chemiluminescent (ECL) protocol with detection using a ChemiGenius Bioimaging System (Syngene, Frederick, MD). Camera exposure was continued until adequate contrast was achieved between the background and protein bands. Membranes were subsequently stained using $0.25 \% \mathrm{w} / \mathrm{v}$ Coomassie brilliant blue followed by destaining with $50 \% \mathrm{v} / \mathrm{v}$ methanol $-10 \% \mathrm{v} / \mathrm{v}$ acetic acid. This was repeated until protein bands were clearly visible against the background. ECL bands were quantified and normalized against the relative protein levels determined by Coomassie blue staining for each individual band to account for variations in the amount of protein loaded between samples.

\subsubsection{Incubations to Stimulate Endogenous Kinases and Phosphatases}

To assess the effects of phosphorylation on the kinetic parameters of KGDC, tissue lysates were incubated in buffers that promoted the activity of one or more protein kinases or phosphatases. Muscle samples were homogenized as before in $\mathrm{pH} 7.2$ homogenate buffer. These samples were then centrifuged at $10,000 \mathrm{rpm}$ for $30 \mathrm{~min}$ at $4{ }^{\circ} \mathrm{C}$ to separate the insoluble fraction from the rest of the homogenate. Samples of $500 \mu \mathrm{L}$ were then run through $5 \mathrm{~mL}$ G-25 Sephadex 
columns pre-equilibrated in OPEN buffer (same components as the homogenate buffer but without EDTA, EGTA, or $\beta$-glycerophosphate) via centrifugation at $2500 \mathrm{rpm}$ for $1 \mathrm{~min}$. This was done to remove any ions or small metabolites present in the samples as well as to remove the inhibitors of kinases and phosphatases present in the homogenate buffer. After transfer into OPEN buffer, protein samples were diluted 1:1 v/v with one of the following solutions (all solutions were prepared in OPEN buffer and thus also contained 10\% v/v glycerol, $25 \mathrm{mM}$ Tris, 5 mM 2-mercaptoethanol, pH 7.2):

- OPEN; 25 mM Tris, 5 mM 2-mercaptoethanol, 10\% glycerol, pH 7.2

- STOP; 0.4 mM EDTA/EGTA, $30 \mathrm{mM} \beta$-glycerophosphate

- All kinases; $10 \mathrm{mM}$ Mg-ATP, $30 \mathrm{mM}$ $\beta$-glycerophosphate, $2 \mathrm{mM}$ cAMP, 2 mM cGMP, $2.6 \mathrm{mM} \mathrm{CaCl}_{2}, 7 \mu \mathrm{g} / \mathrm{mL}$ phorbol myristate acetate, $2 \mathrm{mM}$ AMP, $1 \mathrm{U}$ calmodulin per $\mathrm{mL}$

- All phosphatases; $10 \mathrm{mM} \mathrm{MgCl}, 10 \mathrm{mM} \mathrm{CaCl}_{2}$

- Tyrosine phosphatases; $30 \mathrm{mM} \mathrm{NaF}$

- Serine/Threonine Phosphatases; $10 \mathrm{mM} \mathrm{MgCl} 2,10 \mathrm{mM} \mathrm{CaCl}_{2}, 30 \mathrm{mM} \mathrm{Na}_{3} \mathrm{VO}_{4}$

After dilution in the respective buffers, the homogenates were left overnight to incubate at $4{ }^{\circ} \mathrm{C}$ to allow for the action of selected endogenous protein kinases and phosphatases. The following day, the samples were centrifuged through $5 \mathrm{~mL}$ columns of G-25 Sephadex preequilibrated in homogenate buffer to stop the kinase and phosphatase reactions. These samples were then assayed to determine the affinity for CoA using the enzymatic assay described earlier. 


\subsubsection{Bioinformatics Analysis}

Several bioinformatics tools were used to investigate the potential role of PTMs in regulating the different subunits of the KGDC. Since Richardson's ground squirrel is not genome-sequenced the closest sequenced relative, the 13-lined ground squirrel (13-LGS) or Ictidomys tridecemlineatus, was used as a model to predict potential phosphorylation sites and other PTMs using online resources. The ADPredict online tool required a sequence with a solved protein structure, so Rattus norvegicus in this instance was used to predict potential ADPribosylation sites that were then subsequently checked to see if they were conserved in the 13LGS sequence (Monte, Manelfi, Gemei, Corda, \& Beccari, 2018). ADPredict was used to look for potential ADP-ribosylation sites on OGDH (UniProt entry: Q5XI78) subunits since this protein component exhibited a marked increase in the degree of ADP-ribosylation in the hibernating form of the complex when compared to the control. The rat sequence was then aligned to the 13-LGS sequence (NCBI Reference Sequence: XP_005319302.1) to determine if the predicted sites were conserved between the two species using Clustal Omega multiple sequence alignment software.

NetPhos3.1 was used to predict the likely phosphorylated serine, threonine and tyrosine residues in each of the three components of KGDC (Blom, Gammeltoft, \& Brunak, 1999). The PhosphoSitePlus ${ }^{\circledR}$ (Cell Signaling Technologies) online resource was used to confirm whether or not the predicted phosphorylation events corresponded to any experimentally verified phosphorylation sites on homologous proteins in model mammalian species (Hornbeck et al., 2012). Sequences of 13-LGS were aligned to sequences in which modifications were reported in order to ensure that the sites with experimentally verified evidence were conserved in the ground squirrel sequence. Sites that were found to have both significant experimentally deduced 
evidence and a strong phosphorylation prediction score were considered to be likely candidates for regulatory roles in cases where the protein components exhibited significant differences between euthermic and hibernating states, as seen in the immunoblotting experiments.

\subsubsection{Data and Statistical Analysis}

Data obtained for enzyme kinetic assays were analyzed using a microplate analysis program to assess enzyme velocities (Brooks, 1994) and $K_{m}$ values were calculated using Kinetics 3.51 software (Brooks, 1992). Bar charts and statistical analyses for bar charts were performed using RBioplot software (Zhang \& Storey, 2016). Student's heteroscedastic t-tests were used when comparing euthermic control values to a corresponding hibernating values and were considered significantly different when $p<0.05$. Multiple group comparisons were analyzed for statistical significance using ANOVA and considered different when $\mathrm{p}<0.05$.

\subsection{Results}

\subsubsection{Partial Purification of KGDC}

A partial purification ( $\sim 38$-fold from crude) was accomplished by exploiting the massive size of KGDC. Sequential PEG- 8000 precipitations were used, first at 2\%, then increased to $6 \%$, to precipitate the complex. While only about half of the activity was retained after attempting to resolubilize the $6 \%$ PEG precipitate, only $4.9 \%$ of the total protein from the $2 \%$ PEG supernatant was retained, allowing for a 12.8-fold purification. The enzyme was further purified using gel filtration through Sephacryl S-400. Owing to the large size of KGDC, the complex was eluted in the void volume of the column allowing for further separation from smaller protein components. This chromatography step gave a 3 -fold purification of the $6 \%$ precipitate for the final fold purification of 38 -fold from the crude homogenate (Table 4.1.). Using western blotting, the 
individual protein components of the KGDC were identified in the sample enriched for the complex: OGDH (E1), DLST (E2) and DLD E3 (Fig. 1A, B and C, respectively).

\subsubsection{Enzyme Kinetic Parameters}

KGDC affinity for substrates and cofactors remained consistent between the control and hibernating preparations except for CoA. The $K_{m} \mathrm{CoA}$ for the KGDC from muscle of hibernating animals was between 2-3 times higher than that for the euthermic enzyme at each assay temperatures $\left(5^{\circ}, 22^{\circ}\right.$, and $\left.37^{\circ} \mathrm{C}\right)$ (Table 4.2.). In addition, the $K_{A}$ for $\mathrm{Ca}^{2+}$ ion was significantly higher for the hibernator enzyme compared to euthermic value at the $5{ }^{\circ} \mathrm{C}$ assay temperature. Interestingly, the activating effect of calcium appears to be reduced at lower temperatures with the $K_{A} \mathrm{Ca}^{2+}$ increased by 2.11 fold and 2.21 fold between $22{ }^{\circ} \mathrm{C}$ and $5{ }^{\circ} \mathrm{C}$ for the euthermic and hibernating enzyme preparations, respectively. However, the maximum KGDC activity in crude enzyme preparations was not significantly different between euthermic and hibernating conditions, with values at $5^{\circ} \mathrm{C}(1.2 \pm 0.4$ and $1.9 \pm 0.2 \mathrm{mU} / \mathrm{mg}), 22{ }^{\circ} \mathrm{C}(4.56 \pm 0.6$ and $3.98 \pm$ $0.06 \mathrm{mU} / \mathrm{mg})$ and $37^{\circ} \mathrm{C}(5.3 \pm 1.6$ and $7.39 \pm 0.7 \mathrm{mU} / \mathrm{mg})$, respectively.

\subsubsection{Co-immunoprecipitation and Mass Spectroscopy}

Co-immunoprecipitation was performed on euthermic and hibernating tissue samples after the PEG precipitation step, as previously described. Proteins were bound to antibodies chemically cross-linked to protein-A on agarose beads and were removed from the bead by boiling in SDS-PAGE loading buffer. Polyacrylamide gel electrophoresis and staining with Coomassie Brilliant blue revealed the presence of three distinct protein bands (A, C, D) (Fig. 4.2.) corresponding to the bands seen in the immunoblots shown in Fig. 4.1. An additional band was seen between the DLD band and the OGDH band slightly below the $95-\mathrm{kDa}$ marker. This band was also occurred in the two negative controls that were run (protein-A agarose beads only, 
and a non-specific antibody) and was identified by LC/MS/MS as phosphofructokinase-1 (labeled B in Fig. 4.2.). LC/MS/MS confirmed band A ( 116 kDa) as OGDH, band C ( $\sim 59-\mathrm{kDa})$ as DLD, and band D ( 53kDa) as DLST (Fig. 4.2.).

\subsubsection{Evaluation of Post-translational Modifications}

Immunoblotting of the protein bands obtained via co-immunoprecipitation was performed using a variety of pan-specific antibodies recognizing modified residues. OGDH (E1) displayed a number of alterations to its PTM status between euthermic and hibernation states. OGDH from muscle of hibernating squirrels demonstrated a considerable increase ( 2.2 fold) in the degree of ADP-ribosylation relative to the euthermic control (Fig. 4.3.). The OGDH samples from muscle of hibernating animals also showed a significantly higher degree of lysine succinylation than in the euthermic state (1.5 fold). OGDH from hibernating muscle was also associated with an increase in 1.6-fold increase in tyrosine phosphorylation. Phospho-tyrosine content also increased by 1.9 and 1.5 -fold, respectively, for the DLST (Fig. 4.4.) and DLD (Fig. 4.5.) subunits. Phosphorylation on serine residues was also significantly increased on the DLST (1.8 fold) in the hibernating samples relative to controls (Fig. 4.4.). None of the other PTMs analyzed showed any statistically significant differences between the two states.

\subsubsection{Effects of Phosphatases and Kinases on CoA Affinity}

To assess the importance of the observed phosphorylation changes noted by the immunoblotting, the $K_{m}$ for CoA of KGDC was determined following incubation of crude tissue extracts under conditions that activated or promoted the activity of endogenous protein kinases. The STOP condition contained the normal homogenate buffer (with all inhibitors of kinases and phosphatases present) and these samples showed a similar relationship between $K_{m} \mathrm{CoA}$ in euthermic control versus hibernating conditions as seen for the partially purified complexes; i.e. 
the $K_{m} \mathrm{CoA}$ was significantly higher in the hibernating condition (Fig. 4.6.). Conditions selective for promoting Tyr (Y) phosphatase or Ser/Thr (ST) phosphatase action led to a decrease in the $K_{m}$ for both the control and the hibernating conditions. Conditions favorable for the activity of all phosphatases led to the lowest $\mathrm{K}_{\mathrm{m}}$ of all of the conditions tested for both forms of the enzyme. Oddly, a similar decrease in $K_{m}$ was observed for the OPEN (no inhibitors of kinases or phosphatases) and even occurred under conditions that favored the action of all kinases. In all cases, except the total kinase trials, the hibernating form of the enzyme demonstrated a higher $K_{m}$ CoA than the corresponding euthermic control value.

\subsubsection{Bioinformatics Analysis}

The ADPredict online resource was used to look for potential ADP-ribosylation sites on the OGDH subunit using the Rattus norvegicus protein as the closest genome-sequenced relative of Richardson's ground squirrels and the OGDH sequence from the 13-lined ground squirrel ( $I$. tridecemlineatus) (Appendix B.1.). Using PhosphoSitePlus online resource to look for experimentally verified phosphorylation sites on tyrosine residues demonstrated several possible sites where this modification could occur in the ground squirrel sequence (https://www.phosphosite.org/proteinAction.action?id=9034\&showAllSites=true). One site (Y527) has been shown to be a phosphorylated residue in 9 high-throughput studies and is conserved in the 13-LGS. This was also located in close proximity to a verified succinyl site that is conserved in the ground squirrel (K534). Interestingly, Y527 was not predicted to be phosphorylated by NetPhos3.1 or NetPhos2.0 in either the 13-LGS sequence or a human sequence despite several independent studies showing it to be phosphorylated (Appendix B.1.).

Searching for loci of serine phosphorylation on DLST in the Phospho Site Plus database yielded a single residue that is known by multiple high-throughput studies to be phosphorylated 
(https://www.phosphosite.org/proteinAction.action?id=18684\&showAllSites=true) (Appendix B.2.). This site was conserved in the 13-lined ground squirrel, the closest sequenced relative of the Richardson's ground squirrel. This site (S82) has 10 independent high-throughput studies demonstrating phosphorylation in other mammals recorded on PhosphoSitePlus and is located within the lipoyl binding domain of the enzyme. Using NetPhos 3.1 demonstrated that several different kinases are predicted to phosphorylate this site. Despite the changes noted in tyrosine phosphorylation levels via the western blots, searching the PhosphoSitePlus database for phosphorylated tyrosine residues did not yield any results, although two sites from the $I$. tridecemlineatus sequence were predicted to be potentially phosphorylated by NetPhos 3.1. Interestingly, the tyrosine residue with the highest prediction score was found in an area not conserved in the rat sequence $(\mathrm{Y} 42$, score $=0.828)$.

The only difference noted in the PTM status of DLD between the euthermic control and hibernating states was an increase in phosphorylation on tyrosine residues. Interestingly, numerous high-throughput studies have shown phosphorylation on a specific residue (Y153) in human DLD (https://www.phosphosite.org/siteAction.action?id=12272472) that is conserved in the I. tridecemlineatus sequence (Appendix B.3.).

\subsection{Discussion}

Hibernation is known to cause dramatic alterations to cellular metabolism including an overall reduction in cellular respiration and a shift to consume lipids over carbohydrates (Michener \& Locklear, 1990). While a number of studies have characterized important regulatory steps in the glycolytic pathway of this species, comparatively little research has looked at the control of regulatory steps in the CAC. This study demonstrates significant differences in the kinetic parameters of KGDC from euthermic and hibernating Richardson's 
ground squirrels that suggest that inhibition of this enzyme complex plays an important role in the reduction of metabolic rate seen during bouts of torpor.

Analysis of the kinetic parameters of the KGDC complex between the euthermic control and hibernating states showed few changes between the partially pure complexes. The most notable difference was an increase in the $K_{m}$ for CoA in the hibernating form of the complex (Table 4.2.). This difference was consistent across all three assay temperatures. This suggests that any alterations to the complex at the outset of hibernation would have regulatory significance in reducing the affinity for CoA across a wide range of temperatures such as occur during the course of hibernation. The steady state concentrations of CoA in skeletal muscle mitochondria for this species is currently unknown however within rat liver mitochondria the abundance of CoA has been reported as $1.21 \mu \mathrm{mol} / \mathrm{g}$ (Garland, Shepherd, \& Yates, 1965). This figure is likely much higher than in muscle since various sources have reported that the mammalian abundance of CoA is about 6-fold higher in the liver than in skeletal muscle (Brass, 1994). If the skeletal muscle mitochondrial levels of CoA are assumed to be one sixth the concentration of liver levels and there is approximately $500 \mathrm{mg}$ of protein per $\mathrm{mL}$ in the mitochondrial matrix (Kühlbrandt, 2015), then the CoA concentration within the skeletal muscle mitochondrial matrix can be estimated to be about $0.1 \mathrm{mM}$. While these calculations can only give a rough estimate, they demonstrate that the concentration of CoA in the subcellular space within the mitochondria is likely not much higher or lower than the $K_{m}$ values determined here and as such the changes observed here are likely reflective of significant differences in the functionality of the enzyme complex in vivo. In addition to the decreased affinity for CoA in the hibernating form of the complex, a temperature dependent alteration in the affinity for the activating $\mathrm{Ca}^{2+}$ ions was also observed at $5{ }^{\circ} \mathrm{C}$ where $\mathrm{KGDC}$ from hibernating animals showed a 
reduced affinity for the ion (1.16 fold change) (Table 4.2.). This was in addition to the increased $\mathrm{K}_{\mathrm{A}}$ observed for both the control and the hibernating at $5{ }^{\circ} \mathrm{C}$ relative to the higher temperatures. The significance of temperature in regards to metabolic regulation has been noted in the goldenmantled ground squirrel whose PFK affinity for substrate decreased markedly at lower temperatures, suggesting an inhibition of glycolytic pathways during hibernation (Justin A.

MacDonald \& Storey, 2005). Together this suggests that both alterations to enzyme structure and temperature-dependent effects on enzyme parameters could slow the rate of flux through KGDC to help control mitochondrial metabolism during hibernation. The differences noted between the euthermic control form of the enzyme complex and the hibernating form also suggested that alterations in the PTM status of one or more of the components could be responsible for these changes.

Mitochondrial proteins are subject to a diverse and atypical array of PTMs in comparison to other compartments of the cell (Hofer \& Wenz, 2014). The mitochondria contains a substantial portion of the cellular NAD/NADH coenzyme pool (up to $\sim 70 \%$ ) and this has a profound influence over mitochondrial metabolism both by acting as a substrate for mitochondrial sirtuins which catalyze deacylation reactions and also by acting as the source of ADP ribose which can be ligated to enzymes (Dölle, Rack, \& Ziegler, 2013). The present study demonstrated a greater than 2-fold increase in ADP-ribosylation in OGDH during hibernation compared to the euthermic controls. This modification primarily affects the acidic amino acid residues, aspartate and glutamate, but is known to affect others such as cysteine, arginine, and serine (Liu \& Yu, 2015). ADP-ribosylation may take the form of either a single added ADPribosyl group or result from the addition of a polymeric chain (PARylation) generated by polyADP-ribosyl polymerase activity (PARP). Whereas PARylation is better understood in the 
context of regulation of DNA associated proteins such as histones, it has been more recently established that several PARylated proteins exist in the inner mitochondrial space and are often formed as a result of oxidative stress (Brunyanszki, Szczesny, Virág, \& Szabo, 2016; Pankotai, Lacza, Murányi, \& Szabó, 2009). The techniques employed here did not distinguish between mono-ADP ribosylated proteins or PARylated proteins but the DLD subunit of KGDC has been proposed to be partly responsible for mitochondrial PARP activity (Pankotai et al., 2009). The presence of increased ADP-ribosylation of skeletal muscle OGDH in hibernating squirrels may be indicative of an alteration in $\mathrm{NAD}^{+}$metabolism since the formation of PAR through mitochondrial PARP activity and mono-ADP-ribosyltransferase activity through SIRT4 are dependent on $\mathrm{NAD}^{+}$(Herrero-Yraola et al., 2001). ADP-ribosylation prediction software (ADPredict) was used to demonstrate the presence of 17 potential aspartate and glutamate sites for ADP-ribosylation in a rat sequence with 15 of these sites being conserved in the sequence from 13-lined squirrel (I. tridecemlineatus) (Appendix B.1.) (Monte et al., 2018). To our knowledge, this is the first time that differential protein ADP-ribosylation has been observed for $\mathrm{OGDH}$ and its regulatory significance is unknown. Mono-ADP-ribosylation of a cysteine residue is known to play a role in inhibiting the activity of glutamate dehydrogenase, an important mitochondrial enzyme that effectively bridges the CAC, amino acid metabolism, and the urea cycle (Haigis et al., 2006; Herrero-Yraola et al., 2001). Relative levels of ADP-ribosylation were demonstrated to be reduced in liver GDH from wood frogs in response to whole body freezing, a PTM known to be associated with inhibition of that enzyme (Green \& Storey, 2017; HerreroYraola et al., 2001).

KGDC has also been proposed to play a role as a protein-succinyl transferase and is thought to act in succinylation of proteins involved in the CAC including its own OGDH subunit 
as well as isocitrate dehydrogenase (Gibson et al., 2015). Mitochondrial levels of succinyl-CoA, one of the products of KGDC activity, is thought to be an important cofactor in the succinylation of lysine residues in mitochondrial proteins and may be related in regulating central metabolism (Zhang et al., 2011). The current study demonstrates evidence that the metabolic events surrounding hibernation also lead to increasing levels of succinylation of the OGDH subunit of KGDC. De-succinylation activity in the mitochondria is most commonly associated with SIRT5 activity, which is dependent upon $\mathrm{NAD}^{+}$(Du et al., 2011). While increases in total SIRT activity (measured through deacetylation assays) in I. tridecemlineatus have been observed in the skeletal muscle in response to torpor when compared to euthermic controls, this occurred without a corresponding increase in SIRT5 levels (Rouble \& Storey, 2015). Changes in succinylation of the OGDH subunit seen in the current study are therefore likely to be either the result of an increased succinylation rate that may be due to alterations in levels of available succinyl-CoA or perturbations in the NAD/NADH balance within the mitochondria during hibernation. Indeed, a high-throughput study demonstrated that lysine succinylation occurs in 4 conserved sites of mammalian OGDH in a related ground squirrel species (13-lined ground squirrel; Appendix B.1.), emphasizing the potential regulatory significance of this PTM (Weinert et al., 2013).

The role of phosphorylation in regulating the KGDC is not entirely understood. Although the structurally homologous keto-acid dehydrogenase complex PDC is known to be tightly regulated by phosphorylation on several conserved serine and threonine residues (mediated by pyruvate dehydrogenase kinases 1-4) that inhibit PDC activity and PDC phosphatases that activate, no strong evidence exists for a similar regulation of the KGDC (Yeaman et al., 1978; Zhang, Hulver, McMillan, Cline, \& Gilbert, 2014). Interestingly, a chief player in PDC phosphatase control is the presence of calcium or magnesium ions. These help to activate the 
complex indirectly by promoting the interaction of phosphatases with the complex (Huang et al., 2002). KGDC is also activated by calcium ions although the mechanism is different since the activation is thought to be a result of the binding of $\mathrm{Ca}^{2+}$ to OGDH dimers within the complex, thereby regulating their activity (Denton, 2009; Lawlis \& Roche, 1981). Despite the lack of known roles for KGDC phosphorylation, the results of this study demonstrated an increase in tyrosine phosphorylation on all three components of the complex, as well as an increase of phosphorylation on serine residues on DLST during hibernation (Fig. 4.4.). The increase in DLST phosphorylation may be particularly important in the regulation of this complex since this component is the subunit that directly binds CoA, the affinity for which was noted to be significantly different between the euthermic and hibernating conditions. Serine phosphorylation has been documented by several studies on human tissues, including skeletal muscle, as occurring on a residue in the CoA binding site of DLST (Mertins et al., 2014, 2016; Zhao et al., 2011). This site is conserved in the I. tridecemlineatus sequence, suggesting that the addition of a phosphate group at this location may explain the observed decrease in affinity for CoA of the ground squirrel enzyme from hibernating tissues. Using NetPhos 3.1 phosphorylation prediction, this residue (S82) in the I. tridecemlineatus sequence was strongly predicted to be phosphorylated by an unspecified kinase (prediction score $=0.993$, Appendix B.2.). Increased tyrosine phosphorylation was noted on the E3 (DLD) subunit of KGDC in this study and, using PhosphoSitePlus, a conserved tyrosine residue (Y153) was identified in the ground squirrel sequence that has been listed by numerous high-throughput curated MS data sets as a phosphorylation site (https://www.phosphosite.org/siteAction.action?id=12272472). Tyrosine phosphorylation of the DLD subunit in the PDC is associated with inhibition of the complex and 
since this same subunit is also present in the KGDC, it is likely that a similar action occurs here (Li et al., 2016).

To investigate whether or not these phosphorylation differences had a regulatory role in the function of the KGDC, tissue extracts from euthermic and hibernating squirrel muscle were incubated under conditions favorable for the action of protein kinases or phosphatases and the resulting effects on the affinity for CoA were determined. The results demonstrate a decrease in $K_{m} \mathrm{CoA}$ after incubations that promote tyrosine and serine/threonine phosphatases activities. This could imply that elevated phosphorylation may be associated with an increase in $K_{m}$ for CoA, but the $K_{m}$ CoA was actually observed to be consistently lower in the euthermic control as compared with the hibernating situation under all treatments except for exposure to the activity of multiple kinases. Hence, there must be other factors to consider beyond just reversible phosphorylation in regulating the affinity for $\mathrm{CoA}$, such as the increases in succinylation and ADP-ribosylation of OGDH observed in this study. The lowest $K_{m}$ CoA values for both the euthermic and hibernating enzymes were observed in the samples incubated under conditions promoting the activity of all phosphatases, suggesting that both phosphorylation on threonine/serine residues as well as the less typical tyrosine residues plays an important regulatory role in the activity of the complex. Oddly, stimulation of multiple types of kinases seemed to have the opposite effect than expected since the $K_{m}$ CoA was decreased relative to the STOP conditions. This may be an artefact, however, since the repertoire of kinases that could act upon the KGDC would be different in these in vitro experiments with whole tissue extracts than the situation in vivo where KGDC localization within the mitochondria would limit phosphorylation control over KGDH to only those protein kinases present within the organelle. Several kinases are uniquely localized to mitochondria, notably the mitochondrial protein kinase 
family of which PDKs are members along with other kinases targeting the branched-chain ketoacid dehydrogenase complex (Harris et al., 1995). In addition, various other protein kinases are known to be translocated into the mitochondria under specific conditions such as the activation of insulin signaling pathways or under oxidative stress, making the complement of mitochondrial kinases variable and difficult to predict at any given time (Lim et al., 2016).

The results reported in this study have demonstrated that CAC control is an important aspect of metabolic regulation during the winter months for hibernators. The present study showing a reduction in the affinity of the KGDC for its CoA substrate and the altered PTM status of the KGDC subunits during hibernation suggests that active regulation of the $\mathrm{CAC}$ also occurs and is an underappreciated topic in hibernation physiology.

\section{Declaration of Competing Interest}

The authors declare no conflict of interest.

\section{Acknowledgements}

This study was funded by a Discovery grant (\# 6793) from the Natural Sciences and Engineering Research Council of Canada (NSERC). K.B.S. holds the Canada Research Chair in Molecular Physiology. S.R.G. holds an NSERC Alexander Graham Bell Canada Graduate Scholarship at the doctoral level (CGS-D). The authors thank J.M. Storey for editorial review of this manuscript and providing invaluable insight and guidance. 
Table 4.1. Representative partial purification of the KGDC complex from skeletal muscle of Richardson's ground squirrels.

\begin{tabular}{llllll} 
Step & $\begin{array}{l}\text { Total } \\
\text { Protein } \\
(\mathrm{mg})\end{array}$ & $\begin{array}{l}\text { Total } \\
\text { Activity } \\
(\mathrm{mU})\end{array}$ & Yield (\%) & $\begin{array}{l}\text { Fold } \\
\text { Purification }\end{array}$ & $\begin{array}{l}\text { Specific } \\
\text { Activity } \\
(\mathrm{mU} / \mathrm{mg})\end{array}$ \\
\hline Crude & 106.38 & 705.1 & NA & NA & 6.63 \\
\hline $\begin{array}{l}\text { 2\% PEG } \\
\text { Supernatant }\end{array}$ & 98.05 & 868.63 & 123.2 & 1.34 & 8.86 \\
\hline $\begin{array}{l}\text { 6\% PEG pellet, } \\
\text { respun supernatant }\end{array}$ & 4.78 & 404.52 & 57.4 & 12.78 & 84.68 \\
\hline $\begin{array}{l}\text { Sephacryl S-400 } \\
\text { 1.64 }\end{array}$ & 412.94 & 58.6 & 38.07 & 252.34
\end{tabular}


Table 4.2. Kinetic parameters of partially purified skeletal muscle KGDC from control and hibernating Richardson's ground squirrels at three different temperatures. Data are means of $n=4$ determinations $( \pm \mathrm{SEM})$. '*' represents a statistically significant change between a euthermic parameter and the respective hibernating parameter. "a' shows a statistically significant difference between a parameter measured at $5{ }^{\circ} \mathrm{C}$ and its corresponding value at $22{ }^{\circ} \mathrm{C}$. Statistical testing used the Student's t-test, with $\mathrm{p}<0.05$ accepted as significant.

\begin{tabular}{|c|c|c|}
\hline Parameter & Euthermic & Hibernating \\
\hline \multicolumn{3}{|l|}{$5^{\circ} \mathrm{C}$} \\
\hline$\alpha-\mathrm{KG} \mathrm{K}_{\mathrm{m}}(\mathrm{mM})$ & $0.45 \pm 0.01^{\mathrm{a}}$ & $0.40 \pm 0.04$ \\
\hline NAD $K_{m}(m M)$ & $0.11 \pm 0.01$ & $0.090 \pm 0.009^{\mathrm{a}}$ \\
\hline $\mathrm{CoA} \mathrm{K}_{\mathrm{m}}(\mathrm{mM})$ & $0.028 \pm 0.008$ & $0.067 \pm 0.008^{*}$ \\
\hline $\mathrm{Ca}^{2+} \mathrm{K}_{\mathrm{A}}(\mathrm{mM})$ & $0.268 \pm 0.004^{\mathrm{a}}$ & $0.310 \pm 0.007 * \mathrm{a}$ \\
\hline $\mathrm{V}_{\max }(\mathrm{mU} / \mathrm{mg})$ & $60 \pm 4^{\mathrm{a}}$ & $82 \pm 2^{* a}$ \\
\hline \multicolumn{3}{|l|}{$22^{\circ} \mathrm{C}$} \\
\hline$\alpha-K_{G} K_{m}(m M)$ & $0.29 \pm 0.02$ & $0.38 \pm 0.05$ \\
\hline NAD $K_{m}(m M)$ & $0.19 \pm 0.04$ & $0.30 \pm 0.06$ \\
\hline $\mathrm{CoA} \mathrm{K}_{\mathrm{m}}(\mathrm{mM})$ & $0.023 \pm 0.001$ & $0.075 \pm 0.006^{*}$ \\
\hline $\mathrm{Ca}^{2+} \mathrm{K}_{\mathrm{A}}(\mathrm{mM})$ & $0.127 \pm 0.005$ & $0.140 \pm 0.004$ \\
\hline $\mathrm{V}_{\max }(\mathrm{mU} / \mathrm{mg})$ & $290 \pm 10$ & $300 \pm 10$ \\
\hline \multicolumn{3}{|l|}{$37^{\circ} \mathrm{C}$} \\
\hline$\alpha-K_{\mathrm{m}} \mathrm{K}_{\mathrm{m}}(\mathrm{mM})$ & $0.32 \pm 0.03$ & $0.29 \pm 0.04$ \\
\hline $\mathrm{NAD} \mathrm{K}_{\mathrm{m}}(\mathrm{mM})$ & $0.25 \pm 0.03$ & $0.17 \pm 0.03$ \\
\hline $\operatorname{CoA~} K_{m}(m M)$ & $0.026 \pm 0.003$ & $0.065 \pm 0.006^{*}$ \\
\hline $\mathrm{Ca}^{2+} \mathrm{K}_{\mathrm{A}}(\mathrm{mM})$ & $0.120 \pm 0.002$ & $0.118 \pm 0.004^{\mathrm{a}}$ \\
\hline $\mathrm{V}_{\max }(\mathrm{mU} / \mathrm{mg})$ & $410 \pm 10^{\mathrm{a}}$ & $370 \pm 20^{\mathrm{a}}$ \\
\hline
\end{tabular}



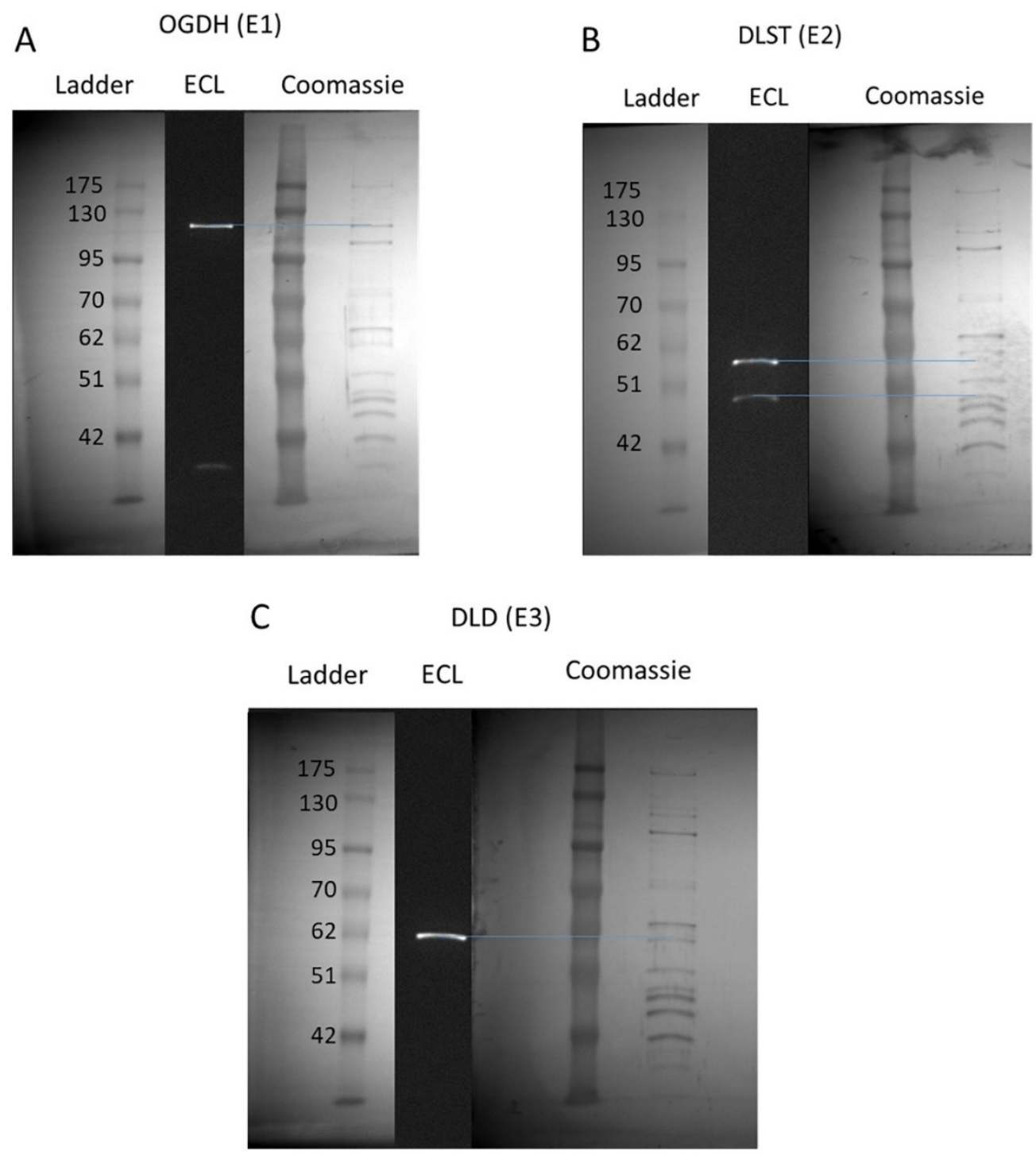

Fig. 4.1. Western blot identification of the component enzymes of the Richardson's ground squirrel $\alpha-K G D H$ complex from euthermic muscle after PEG precipitation and size exclusion chromatography (S-400) enrichment of the enzyme. Molecular weight protein standards shown on the left demonstrate the same membrane positioned identically as compared to the luminescent picture to its right, which were then aligned with the total protein stained Coomassie image. (A) Partially purified KGDC probing for OGDH (E1). (B) Partially purified KGDC probing for the DLST (E2) subunit. (C) Partially purified KGDC probing for the DLD (E3) subunit. 


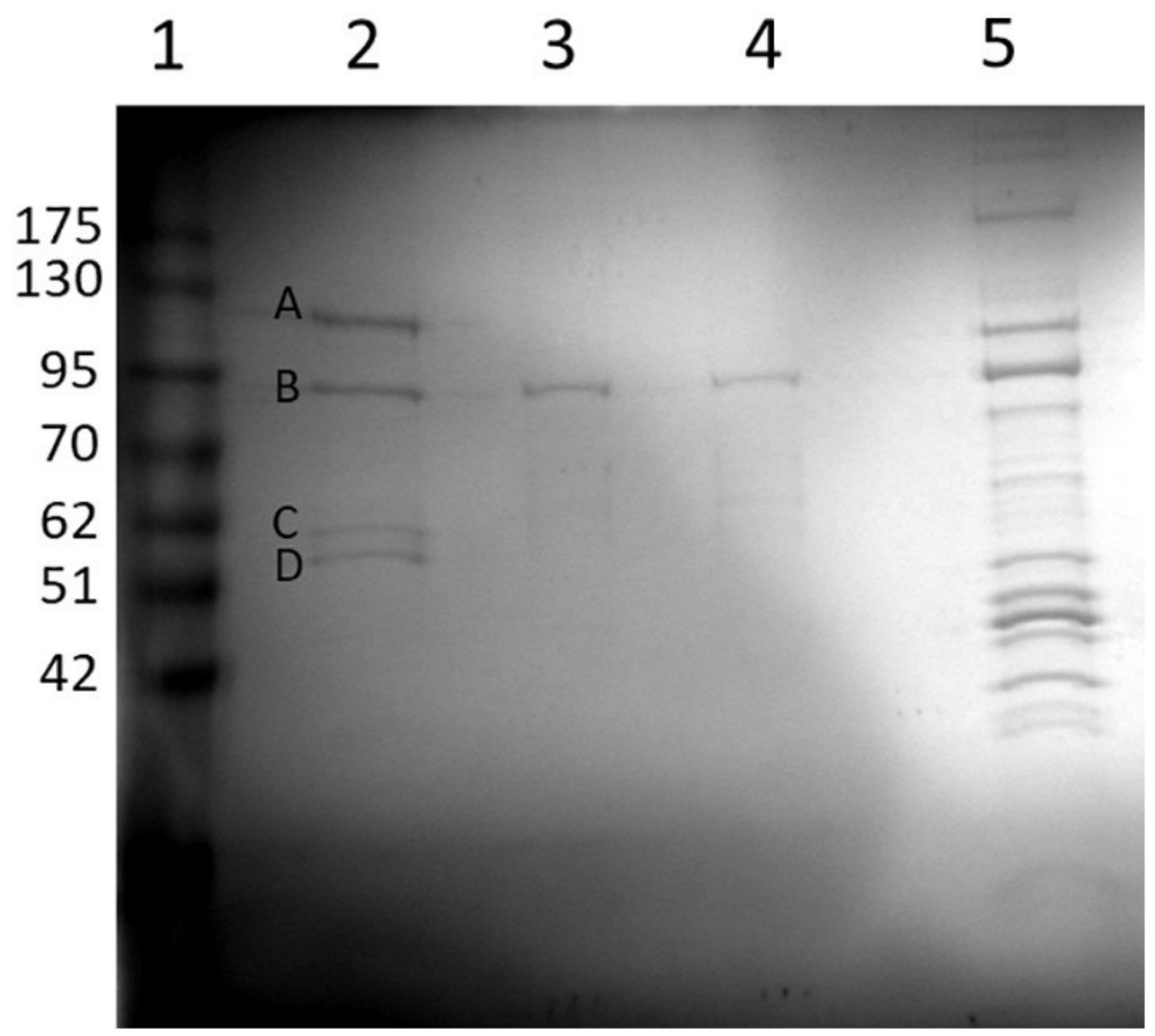

Fig. 4.2. Coomassie stained gel showing purification of KGDC by co-immunoprecipitation (CoIP) of the OGDH (E1) subunit from control Richardson's ground squirrel skeletal muscle. Lanes are as follows: (1) Protein molecular weight ladder (GeneDirex 10.5-175 kDa), (2) Co-IP with anti-OGDH on protein-A agarose beads, (3) Co-IP with non-specific mouse IgG on protein Aagarose beads, (4) Co-IP only protein A-agarose beads, (5). Supernatant from Co-IP using antiOGDH antibody. The labeled bands were identified with mass spectrometry corresponding to; A: OGDH, B: phosphofructokinase, C: DLD, D: DLST. 


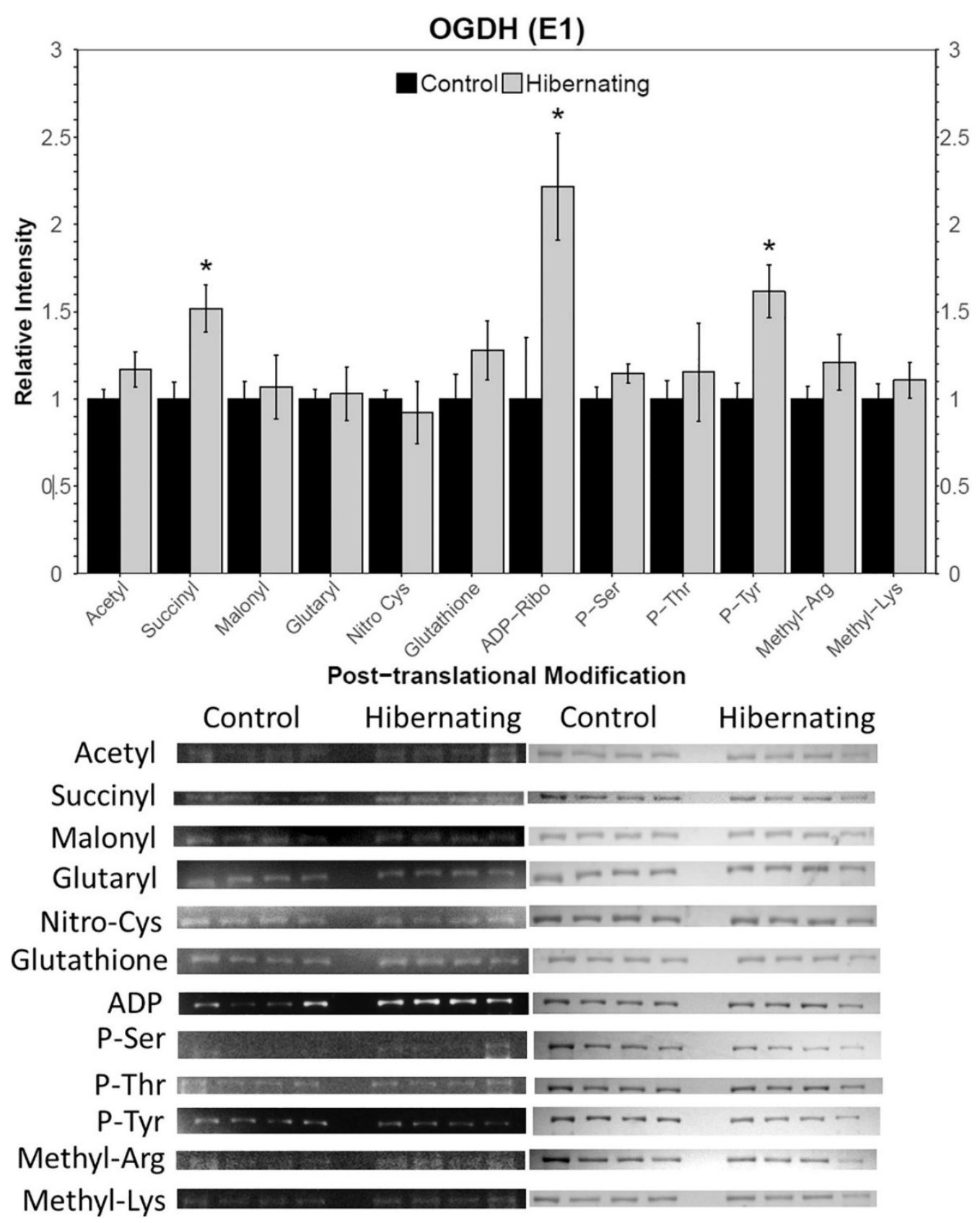

Fig. 4.3. Post-translational modifications of OGDH (E1 subunit of the KGDC) comparing control euthermic muscle samples to hibernating following Co-IP to isolate the protein. Relative luminescence intensities were standardized against the respective total protein signal obtained from Coomassie blue staining. Blots are shown below the graph with the left panel showing the ECL and the right panel the Coomassie stain for the band of interest. Data are means of $4 \pm$ SEM. ' $*$ ' indicates that the hibernating value was determined to be statistically different from the corresponding control euthermic value by Student's t-test, $\mathrm{p}<.05$. 


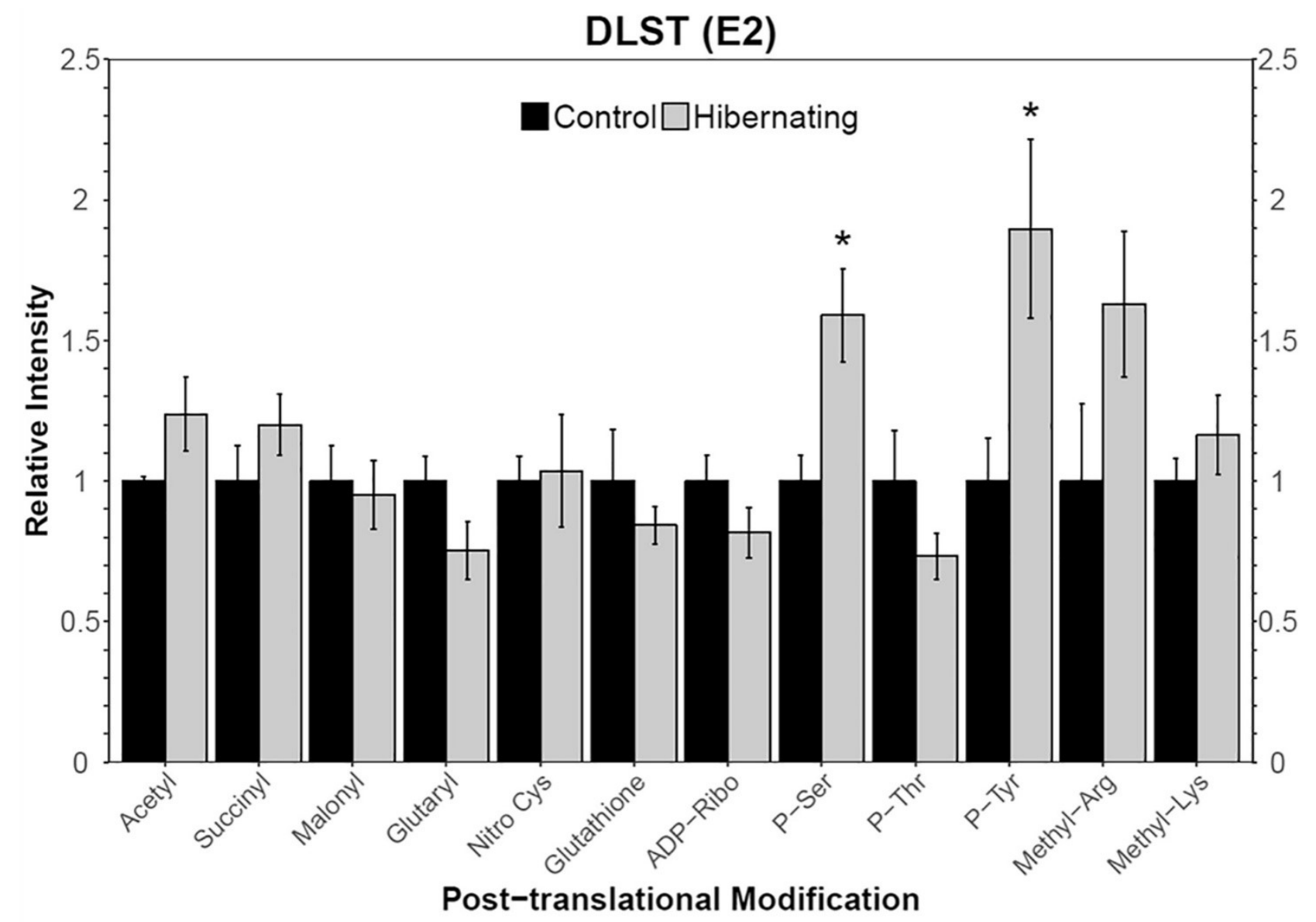

Control Hibernating Control Hibernating

Acetyl

Succinyl

Malonyl

Glutaryl

Nitro-Cys

Glutathione

ADP-Ribo

P-Ser

P-Thr

P-Tyr

Methyl-Arg

Methyl-Lys

Fig. 4.4. Post-translational modifications of DLST (E2 subunit of the KGDC) comparing control euthermic muscle samples to hibernating following Co-IP. All other information as in Fig. 4.3. 
DLD (E3)

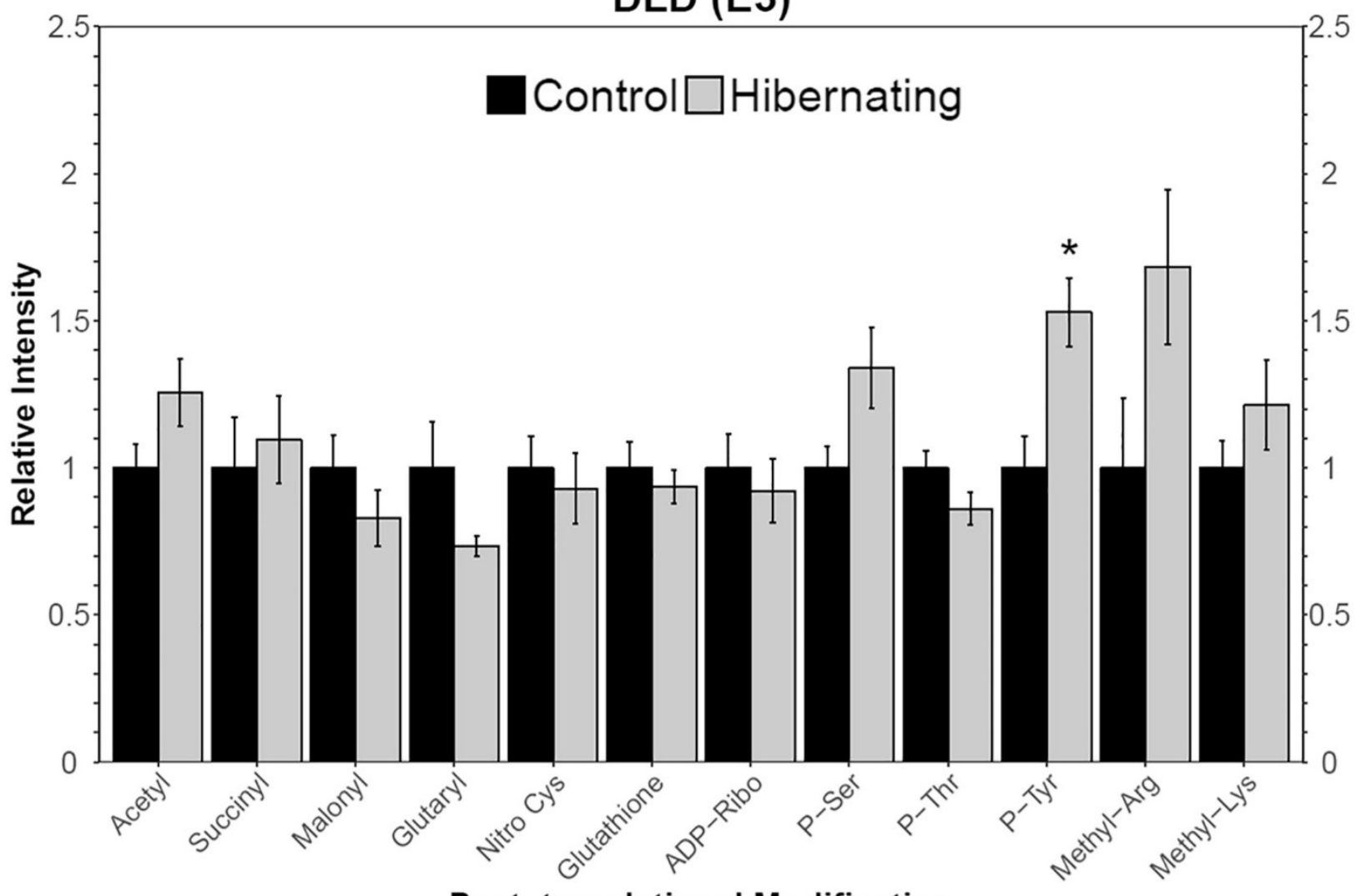

Post-translational Modification
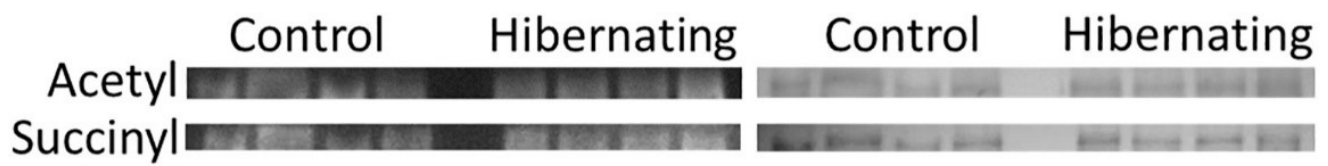

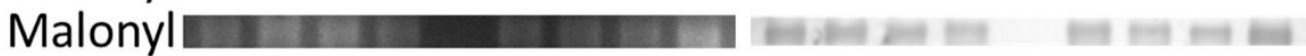

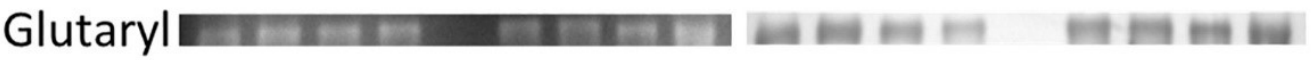

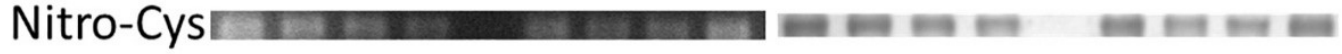

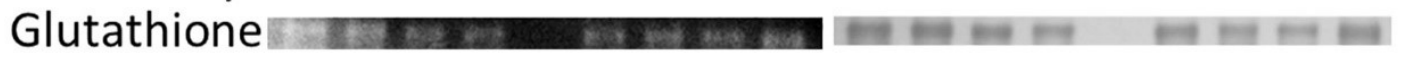

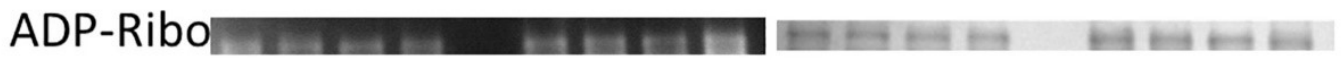

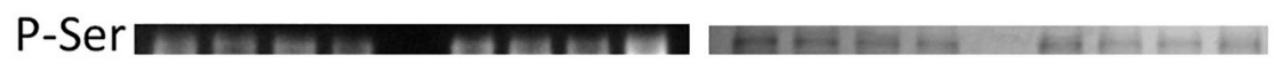

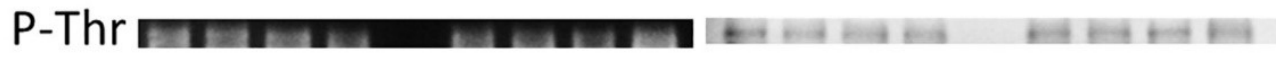

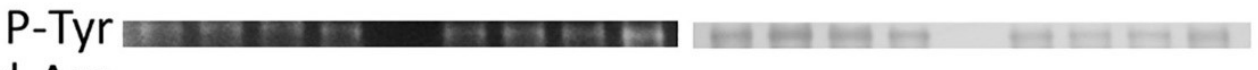

Methyl-Arg

Methyl-Lys

Fig. 4.5. Post-translational modifications of DLD (E3 subunit of the KGDC) comparing control euthermic muscle samples to hibernating following Co-IP. All other information as in Fig. 4.3. 


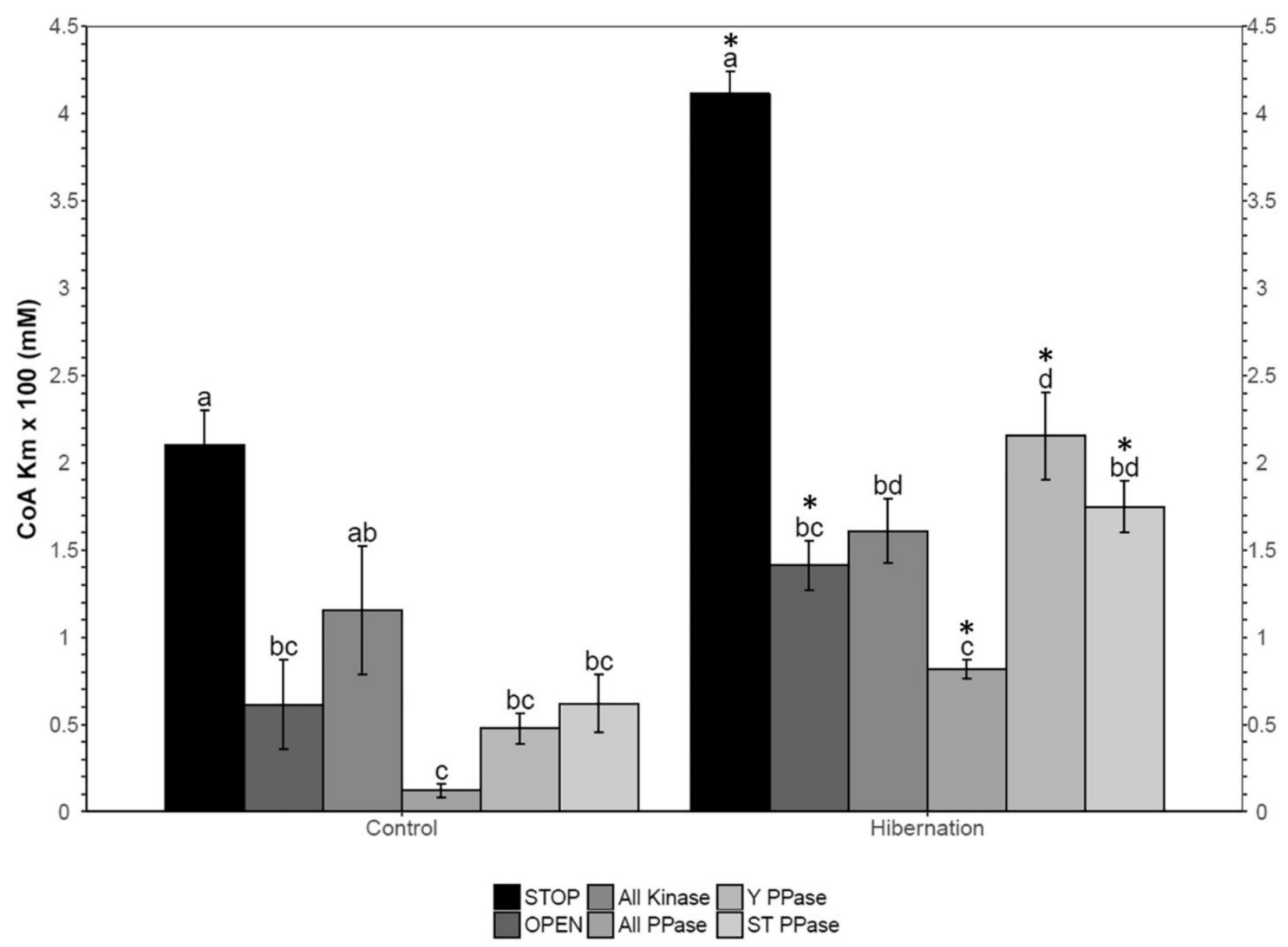

Fig. 4.6. The effects of incubating crude muscle extracts under conditions stimulating endogenous protein kinases or phosphatases on the $\mathrm{K}_{\mathrm{m}} \mathrm{CoA}$ of KGDC. Conditions are as follows: STOP (inhibitors of both kinases and phosphatases present); OPEN (no inhibitors of kinases or phosphatases present); All kinase (conditions stimulating the activity of protein kinase A, protein kinase G, protein kinase C, AMP-activated protein kinase, and calcium-calmodulin protein kinase); All PPase (conditions favorable for the activity of a variety of phosphatases); Y PPase (conditions favorable for tyrosine phosphatase activity while inhibiting serine/threonine phosphatases); ST PPase (conditions favorable for serine/threonine phosphatase activity while inhibiting tyrosine phosphatases). Statistical analysis used one-way ANOVA with Tukey's posthoc test; within each condition (control, hibernation) data sharing the same letters (a-d) are not significantly different from each other, $p<.05$. Student's $t$-tests were used to compare values between corresponding control and hibernating parameters; ' $*$ ' indicates a significant difference, $\mathrm{p}<.05$ ). 


\section{References Chapter 4}

Abnous, K., \& Storey, K. B. (2008). Skeletal muscle hexokinase: Regulation in mammalian hibernation. Molecular and Cellular Biochemistry, 319(1-2), 41-50. https://doi.org/10.1007/s11010-008-9875-5

Blom, N., Gammeltoft, S., \& Brunak, S. (1999). Sequence and structure-based prediction of eukaryotic protein phosphorylation sites. Journal of Molecular Biology, 294(5), 1351-1362. https://doi.org/10.1006/jmbi.1999.3310

Brass, E. P. (1994). Overview of coenzyme A metabolism and its role in cellular toxicity. Chemico-Biological Interactions, 90(3), 203-214. https://doi.org/10.1016/00092797(94)90010-8

Brooks, S. P. (1994). A program for analyzing enzyme rate data obtained from a microplate reader. Biotechniques, 17(6), 1154-1161. Retrieved from http://www.ncbi.nlm.nih.gov/entrez/query.fcgi? $\mathrm{cmd}=$ Retrieve $\& \mathrm{db}=\mathrm{PubMed} \& \mathrm{dopt}=\mathrm{Citation}$ \&list uids $=7873186$

Brooks, S. P. J. (1992). A simple computer program with statistical tests for the analysis of enzyme kinetics. BioTechniques, 13(6), 906-911. Retrieved from http://www.ncbi.nlm.nih.gov/pubmed/1476744

Brooks, S. P. J., \& Storey, K. B. (1992). Mechanisms of glycolytic control during hibernation in the ground squirrel Spermophilus lateralis. Journal of Comparative Physiology B, 162(1), 23-28. https://doi.org/10.1007/BF00257932

Brunyanszki, A., Szczesny, B., Virág, L., \& Szabo, C. (2016). Mitochondrial poly(ADP-ribose) polymerase: The Wizard of Oz at work. Free Radical Biology and Medicine, 100, 257-270. https://doi.org/10.1016/j.freeradbiomed.2016.02.024

Childers, C. L., \& Storey, K. B. (2016). Post-translational regulation of hexokinase function and protein stability in the aestivating frog Xenopus laevis. The Protein Journal, 35(1), 61-71. https://doi.org/10.1007/s10930-016-9647-0

Chinopoulos, C. (2013). Which way does the citric acid cycle turn during hypoxia? The critical role of $\alpha$-ketoglutarate dehydrogenase complex. Journal of Neuroscience Research, 91(8), 1030-1043. https://doi.org/10.1002/jnr.23196

Cooney, G. J., Taegtmeyer, H., \& Newsholme, E. A. (1981). Tricarboxylic acid cycle flux and enzyme activities in the isolated working rat heart. The Biochemical Journal, 200(3), 701-3. https://doi.org/10.1042/bj2000701

Denton, R. M. (2009). Regulation of mitochondrial dehydrogenases by calcium ions. Biochimica et Biophysica Acta (BBA) - Bioenergetics, 1787(11), 1309-1316.

https://doi.org/10.1016/j.bbabio.2009.01.005 
Dölle, C., Rack, J. G. M., \& Ziegler, M. (2013). NAD and ADP-ribose metabolism in mitochondria. FEBS Journal, 280(15), 3530-3541. https://doi.org/10.1111/febs.12304

Du, J., Zhou, Y., Su, X., Yu, J. J., Khan, S., Jiang, H., ... Lin, H. (2011). Sirt5 is a NADdependent protein lysine demalonylase and desuccinylase. Science, 334, 806-809. https://doi.org/10.1126/science.1207861

Garland, P., Shepherd, D., \& Yates, D. (1965). Steady-state concentrations of coenzyme A, acetyl-coenzyme A and long-chain fatty acyl-coenzyme A in rat-liver mitochondria oxidizing palmitate. Biochemical Journal, 97(2), 587-594. https://doi.org/10.1042/bj0970587

Geiser, F. (1988). Reduction of metabolism during hibernation and daily torpor in mammals and birds: temperature effect or physiological inhibition? Journal of Comparative Physiology B, 158(1), 25-37. https://doi.org/10.1007/BF00692726

Gibson, G. E., Xu, H., Chen, H. L., Chen, W., Denton, T. T., \& Zhang, S. (2015). Alphaketoglutarate dehydrogenase complex-dependent succinylation of proteins in neurons and neuronal cell lines. Journal of Neurochemistry, 134(1), 86-96. https://doi.org/10.1111/jnc.13096

Green, S. R., \& Storey, K. B. (2017). Regulation of glutamate dehydrogenase (GDH) in response to whole body freezing in wood frog liver linked to differential acetylation and ADPribosylation. Archives of Biochemistry and Biophysics, 636, 90-99. https://doi.org/10.1016/j.abb.2017.10.010

Haigis, M. C., Mostoslavsky, R., Haigis, K. M., Fahie, K., Christodoulou, D. C., Murphy, A. J., ... Guarente, L. (2006). SIRT4 inhibits glutamate dehydrogenase and opposes the effects of calorie restriction in pancreatic b cells. Cell, 126(5), 941-954. https://doi.org/10.1016/j.cell.2006.06.057

Harris, R. A., Popov, K. M., Zhao, Y., Kedishvili, N. Y., Shimomura, Y., \& Crabb, D. W. (1995). A new family of protein kinases- The mitochondrial protein kinases. Advances in Enzyme Regulation, 35(C). https://doi.org/10.1016/0065-2571(94)00020-4

Herrero-Yraola, A., Bakhit, S. M. A., Franke, P., Weise, C., Schweiger, M., Jorcke, D., \& Ziegler, M. (2001). Regulation of glutamate dehydrogenase by reversible ADP-ribosylation in mitochondria. EMBO Journal, 20(10), 2404-2412. https://doi.org/10.1093/emboj/20.10.2404

Hofer, A., \& Wenz, T. (2014). Post-translational modification of mitochondria as a novel mode of regulation. Experimental Gerontology, 56, 202-220. https://doi.org/10.1016/j.exger.2014.03.006

Hornbeck, P. V., Kornhauser, J. M., Tkachev, S., Zhang, B., Skrzypek, E., Murray, B., ... Sullivan, M. (2012). PhosphoSitePlus: A comprehensive resource for investigating the 
structure and function of experimentally determined post-translational modifications in man and mouse. Nucleic Acids Research, 40(D1). https://doi.org/10.1093/nar/gkr1122

Huang, B., Gudi, R., Wu, P., Harris, R. A., Hamilton, J., \& Popov, K. M. (2002). Isoenzymes of Pyruvate Dehydrogenase Phosphatase. Journal of Biological Chemistry, 273(28), 1768017688. https://doi.org/10.1074/jbc.273.28.17680

Izard, T., Aevarsson, A., Allen, M. D., Westphal, a H., Perham, R. N., de Kok, A., \& Hol, W. G. J. (1999). Principles of quasi-equivalence and Euclidean geometry govern the assembly of cubic and dodecahedral cores of pyruvate dehydrogenase complexes. Proceedings of the National Academy of Sciences, 96(4), 1240-1245. https://doi.org/10.1073/pnas.96.4.1240

Kühlbrandt, W. (2015). Structure and function of mitochondrial membrane protein complexes. BMC Biology. https://doi.org/10.1186/s12915-015-0201-x

Lawlis, V. B., \& Roche, T. E. (1981). Regulation of Bovine Kidney $\alpha$-Ketoglutarate Dehydrogenase Complex by Calcium Ion and Adenine Nucleotides, Effects on S0.5 for aKetoglutarate. Biochemistry, 20(9), 2512-2518. https://doi.org/10.1021/bi00512a023

Li, X., Wang, L., Li, Y., Fu, J., Zhen, L., Yang, Q., ... Zhang, Y. (2016). Tyrosine phosphorylation of dihydrolipoamide dehydrogenase as a potential cadmium target and its inhibitory role in regulating mouse sperm motility. Toxicology, 357-358, 52-64. https://doi.org/10.1016/j.tox.2016.06.003

Lim, S., Smith, K. R., Lim, S.-T. S., Tian, R., Lu, J., \& Tan, M. (2016). Regulation of mitochondrial functions by protein phosphorylation and dephosphorylation. Cell \& Bioscience, 6(1), 25. https://doi.org/10.1186/s13578-016-0089-3

Liu, C., \& Yu, X. (2015). ADP-Ribosyltransferases and Poly ADP-Ribosylation. Current Protein \& Peptide Science, 16(6), 491-501. https://doi.org/10.2174/1389203716666150504122435

MacDonald, J. A., \& Storey, K. B. (1998). cAMP-dependent protein kinase from brown adipose tissue: Temperature effects on kinetic properties and enzyme role in hibernating ground squirrels. Journal of Comparative Physiology - B Biochemical, Systemic, and Environmental Physiology, 168(7), 513-525. https://doi.org/10.1007/s003600050172

MacDonald, J. A., \& Storey, K. B. (2005). Temperature and phosphate effects on allosteric phenomena of phosphofructokinase from a hibernating ground squirrel (Spermophilus lateralis). FEBS Journal, 272(1), 120-128. https://doi.org/10.1111/j.14321033.2004.04388.x

Mertins, P., Mani, D. R., Ruggles, K. V., Gillette, M. A., Clauser, K. R., Wang, P., .. Carr, S. A. (2016). Proteogenomics connects somatic mutations to signalling in breast cancer. Nature, 534(7605), 55-62. https://doi.org/10.1038/nature18003

Mertins, P., Yang, F., Liu, T., Mani, D. R., Petyuk, V. A., Gillette, M. A., ... Carr, S. A. (2014). Ischemia in tumors induces early and sustained phosphorylation changes in stress kinase 
pathways but does not affect global protein levels. Molecular and Cellular Proteomics, 13(7), 1690-1704. https://doi.org/10.1074/mcp.M113.036392

Michener, G. R., \& Locklear, L. (1990). Over-Winter Weight Loss by Richardson's Ground Squirrels in Relation to Sexual Differences in Mating Effort. Journal of Mammalogy, 71(4), 489-499. https://doi.org/10.2307/1381787

Monte, M. Lo, Manelfi, C., Gemei, M., Corda, D., \& Beccari, A. R. (2018). ADPredict: ADPribosylation site prediction based on physicochemical and structural descriptors. Bioinformatics, 34(15), 2566-2574. https://doi.org/10.1093/bioinformatics/bty159

Pankotai, E., Lacza, Z., Murányi, M., \& Szabó, C. (2009). Intra-mitochondrial poly(ADPribosyl)ation: Potential role for alpha-ketoglutarate dehydrogenase. Mitochondrion, 9(2), 159-164. https://doi.org/10.1016/j.mito.2009.01.013

Reed, L. J. (1974). Multienzyme complexes. Accounts of Chemical Research, 7(2), 40-46. https://doi.org/10.1021/ar50074a002

Rouble, A. N., \& Storey, K. B. (2015). Characterization of the SIRT family of NAD+-dependent protein deacetylases in the context of a mammalian model of hibernation, the thirteen-lined ground squirrel. Cryobiology, 71(2), 334-343. https://doi.org/10.1016/j.cryobiol.2015.08.009

Searle, B. C. (2010). Scaffold: a bioinformatic tool for validating MS/MS-based proteomic studies. Proteomics, 10(6), 1265-9. https://doi.org/10.1002/pmic.200900437

Shi, Q., Xu, H., Yu, H., Zhang, N., Ye, Y., Estevez, A. G., ... Gibson, G. E. (2011). Inactivation and reactivation of the mitochondrial $\alpha$-ketoglutarate dehydrogenase complex. Journal of Biological Chemistry, 286(20), 17640-17648. https://doi.org/10.1074/jbc.M110.203018

Wang, L. C. H. (1979). Time patterns and metabolic rates of natural torpor in the Richardson's ground squirrel. Canadian Journal of Zoology, 57(2), 149-155. https://doi.org/10.1139/z79012

Wang, L. C. H., \& Lee, T. F. (2011). Torpor and Hibernation in Mammals: Metabolic, Physiological, and Biochemical Adaptations. In Comprehensive Physiology (pp. 507-532). Hoboken, NJ, USA: John Wiley \& Sons, Inc. https://doi.org/10.1002/cphy.cp040122

Weinert, B. T., Schölz, C., Wagner, S. A., Iesmantavicius, V., Su, D., Daniel, J. A., \& Choudhary, C. (2013). Lysine succinylation is a frequently occurring modification in prokaryotes and eukaryotes and extensively overlaps with acetylation. Cell Reports, 4(4), 842-851. https://doi.org/10.1016/j.celrep.2013.07.024

Yeaman, S. J., Hutcheson, E. T., Roche, T. E., Pettit, F. H., Brown, J. R., Reed, L. J., ... Dixon, G. H. (1978). Sites of phosphorylation on pyruvate dehydrogenase from bovine kidney and heart. Biochemistry, 17(12), 2364-2370. https://doi.org/10.1021/bi00605a017 
Zhang, J., \& Storey, K. B. (2016). RBioplot: an easy-to-use R pipeline for automated statistical analysis and data visualization in molecular biology and biochemistry. PeerJ, 4, e2436. https://doi.org/10.7717/peerj.2436

Zhang, S., Hulver, M. W., McMillan, R. P., Cline, M. A., \& Gilbert, E. R. (2014). The pivotal role of pyruvate dehydrogenase kinases in metabolic flexibility. Nutrition \& Metabolism, 11(1), 10. https://doi.org/10.1186/1743-7075-11-10

Zhang, Z., Tan, M., Xie, Z., Dai, L., Chen, Y., \& Zhao, Y. (2011). Identification of lysine succinylation as a new post-translational modification. Nature Chemical Biology, 7(1), 5863. https://doi.org/10.1038/nchembio.495

Zhao, X., León, I. R., Bak, S., Mogensen, M., Wrzesinski, K., Højlund, K., \& Jensen, O. N. (2011). Phosphoproteome analysis of functional mitochondria isolated from resting human muscle reveals extensive phosphorylation of inner membrane protein complexes and enzymes. Molecular and Cellular Proteomics, 10(1).

https://doi.org/10.1074/mcp.M110.000299 


\section{Chapter 5: Regulation of Muscle GDH during Hibernation}




\begin{abstract}
Mammalian hibernation is a complex phenomenon mediated through multiple levels of metabolic control, one of which is the post-translational modification of enzymes to accommodate the changes in energetic demands. Glutamate dehydrogenase (GDH) catalyzes a reaction that helps to bridge amino acid metabolism and the citric acid cycle (CAC) by oxidizing glutamate to produce the CAC intermediate $\alpha$-ketoglutarate. Purification of GDH from the muscle tissue of euthermic and hibernating Richardson's ground squirrels (Urocitellus richardsonii) was performed to assess the kinetic parameters and to characterize the relative levels of posttranslational modifications. The results demonstrate a few subtle but significant differences in the kinetic parameters of GDH between the euthermic and hibernating conditions. Notably a decrease in the $I_{50}(0.8$-fold) for the inhibitor GTP was present in the glutamateconsuming direction of the reaction in the hibernating form of the enzyme. Additionally, the $K_{m}$ glutamate for euthermic GDH rose substantially at $8{ }^{\circ} \mathrm{C}$ compared with 22 and $37^{\circ} \mathrm{C}$, while the hibernating $K_{m}$ remained constant suggesting that the hibernating enzyme may be modified in some way to maintain activity at low temperatures. An increase in the level of ADP-ribosylation was noted for the hibernating form of GDH relative to the euthermic controls. This increase in ADP-ribosylation was unexpected because typically this post-translational modification is associated with inhibition of GDH, which was not observed here. This suggests a different locus of modification that may have produced the effects observed here in the kinetic parameters.
\end{abstract}




\subsection{Introduction}

Richardson's ground squirrels (Urocitellus richardsonii) are small mammalian obligate hibernators that inhabit the prairies of western Canada and northern USA. These animals actually spend the majority of their lives in hibernation, averaging $\sim 8.5$ months of the year. Hibernation is a common survival strategy for many small endotherms that inhabit temperate and arctic climates since, for much of the year, low ambient temperatures and reduced food access make it difficult to maintain the high metabolic rates needed for homeothermy. During torpor, these animals typically maintain a minimum body temperature between $2-3{ }^{\circ} \mathrm{C}$ with reduced vital signs such as heart rate and breathing rate that allow them to reduce their metabolic expenditures during the long hibernation period by at least 88\% (Wang, 1979; Wang \& Lee, 1996). In addition to the dramatic reduction in metabolic rate, a pronounced shift in fuel usage also occurs during the hibernating season shifting away from dietary carbohydrates processed via glycolysis to the consumption of body white and brown fat stores accumulated over the summer feeding period (Michener, 1998). The profound reduction in metabolic rate and the shift to lipid catabolism to maintain basal physiological processes suggests that extensive controls exist to regulate the core energy producing reactions of cells.

The citric acid cycle (CAC) is a central metabolic pathway that is critical for both catabolic and anabolic reactions, although it is most notable as a main source of the NADH reducing equivalents that are used to shuttle electrons into the electron transport chain (ETC) and thereby power oxidative phosphorylation to produce ATP. In this pathway the breakdown product of glycolysis and fatty acid $\beta$-oxidation, acetyl coenzyme $\mathrm{A}$, is condensed with oxaloacetate to form citrate through the action of the enzyme citrate synthase and is subsequently oxidized to produce $\mathrm{NADH}, \mathrm{CO}_{2}$ and to reform oxaloacetate. Interestingly, only two reactions in 
the CAC are irreversible, those catalyzed by citrate synthase and $\alpha$-ketoglutarate dehydrogenase, and likely form important targets for regulation as they commit metabolites in this cycle to more specific metabolic fates (Chinopoulos, 2013). Whereas much of the work examining the regulation of these critical enzymes has focused on the importance of feedback inhibition by metabolites such as ATP and NADH, regulation of the enzymes may also depend on extrinsic factors such as posttranslational modifications. Citrate synthase is a target for a specific methyl transferase that inhibits its activity by lysine methylation in the active site and recently, the authors have demonstrated altered functional and post-translational properties of the $\alpha$ ketoglutarate complex in the same animal and tissue studied here (Green \& Storey, 2020; Małecki et al., 2017; Rhein, Carroll, Ding, Fearnley, \& Walker, 2017). Asides from regulation within the $\mathrm{CAC}$, the upstream steps that directly feed into this pathway are also regulated. For example, the pyruvate dehydrogenase complex catalyzes the reaction bridging glycolysis and the $\mathrm{CAC}$ and its inhibition through post-translational modification is well-characterized (Green \& Storey, 2021; Hadj-Moussa, Green, \& Storey, 2018). This current study examines glutamate dehydrogenase (GDH), an important mitochondrial enzyme that shunts $\alpha$-ketoglutarate (KG) into the CAC from glutamate.

The breakdown of protein is a metabolically complex process owing to the differing metabolic fates of the proteinogenic amino acids. Amino acids can be broadly classed into two different categories related to their ultimate metabolic fates: ketogenic, which can be directly converted into acetyl-CoA, and glucogenic, which can be converted into glucose through gluconeogenesis usually in the liver (Ruderman, 1975). Glutamate (Glu) serves as a common intermediate in the catabolism of numerous amino acids including arginine, proline, histidine and glutamine (Brosnan, 2000). GDH connects amino acid metabolism into the CAC by converting 
Glu into $\mathrm{KG}$ through oxidation via $\mathrm{NAD}^{+}$with the release of $\mathrm{NH}_{4}^{+}$according to the following mechanism:

$$
\text { Glutamate }+\mathrm{H}_{2} \mathrm{O}+\mathrm{NAD}^{+} \rightarrow \alpha \text {-ketoglutarate }+\mathrm{NH}_{4}{ }^{+}+\mathrm{NADH}+\mathrm{H}^{+}
$$

This reaction is reversible in vitro, but in vivo the concentrations of $\mathrm{NH}_{4}{ }^{+}$tend to be far lower than needed for substantial activity in this direction. A notable exception occurs in a certain subset of hepatocytes where Glu can be synthesized from free ammonium ion (Spanaki \& Plaitakis, 2012). Ammonium produced from this reaction is metabolized in the liver to form urea in mammals and various other terrestrial organisms thereby reducing its toxicity so that it can be excreted in higher concentrations in the urine.

The role of GDH regulation in Richardson's ground squirrels during hibernation was previously explored in liver. That study demonstrated that GDH in hibernating animals was associated with greater maximal enzyme activity $\left(V_{\max }\right)$, higher Glu affinity, and greater sensitivity to ADP, as compared with euthermic active squirrels. Additionally, the hibernating form of the enzyme showed a slightly higher phosphorylation state indicating that there is likely post-translational control of this enzyme associated with hibernation (Bell \& Storey, 2010). This expanded upon earlier work that demonstrated that inhibition due to GTP was most noticeable in the euthermic form of the enzyme at $37^{\circ} \mathrm{C}$ as compared to the hibernator at $5{ }^{\circ} \mathrm{C}$, suggesting a relief of inhibition to allow for GDH to continue Glu oxidation during hibernation (Thatcher \& Storey, 2001). In mammals, GDH has been studied mostly in the liver and is often thought of as an upstream step to understanding urea cycle regulation since the ammonium generated there can be directly shunted into the urea cycle, which only occurs in the liver (Green \& Storey, 2017). However, GDH is also present in skeletal muscle where it plays a role in catabolizing amino 
acids and can supplement the $\mathrm{CAC}$ by providing $\mathrm{KG}$ for oxidation. The role of GDH in muscle is to manage amino acids and is understood to be regulated through feed-forward activation by branched chain amino acids such as leucine as well as alanine (Palaiologos \& Felig, 1978; Zhou \& Thompson, 1996). Post-translational modifications (PTMs) have been associated with the regulation of GDH functional parameters through several different mechanisms including ADPribosylation and acetylation of lysine residues (Aleshin et al., 2020; Green \& Storey, 2017; Herrero-Yraola et al., 2001). The present study examined the kinetic properties of GDH in the skeletal muscle of euthermic and hibernating $U$. richardsonii and compared the status of several PTMs on purified enzymes.

\subsection{Materials and Methods}

\subsubsection{Animal Treatments}

Richardson's ground squirrels (Urocitellus richardsonii) used in this study were collected near Calgary, Alberta in September and moved to an animal care facility at the University of Calgary where they were housed in separate cages. Animals were kept on a day-night cycle of 10 h light followed by $14 \mathrm{~h}$ dark at $22{ }^{\circ} \mathrm{C}$ for 8 weeks with food freely provided to the animals. Control euthermic animals were sampled from this group. Animals designated for the hibernation group were then exposed to reduced hours of daylight ( $8 \mathrm{~h}$ light, $16 \mathrm{~h}$ dark) and cooling of temperature down to $4{ }^{\circ} \mathrm{C}$ for $24 \mathrm{~h}$. After $24 \mathrm{~h}$ of these conditions, food was taken away from the hibernation group to induce them to enter torpor. Animals were observed over the following week and any that did not enter hibernation were removed from the experiment. After entrance into hibernation, all animals were held under torpid conditions for at up to 7 days before euthanizing. Euthermic squirrels were kept at $22{ }^{\circ} \mathrm{C}$ throughout. Euthanasia was performed via decapitation and tissues were subsequently rapidly dissected, flash frozen in liquid nitrogen and 
then shipped on dry ice to Carleton University. Tissues were immediately stored in a $-80{ }^{\circ} \mathrm{C}$ freezer until use in the experiments. All animal treatments were approved by the Animal Care Committee and abided by the guidelines set down by the Canadian Council on Animal Care.

\subsubsection{Enzyme Purification}

Richardson's ground squirrel thigh muscle samples were quickly weighed and homogenized 1:5 w:v in ice-cold buffer A ( $25 \mathrm{mM}$ MES buffer $\mathrm{pH} 6.0$ containing $2 \mathrm{mM}$ EDTA, 2 mM EGTA, $10 \mathrm{mM} \beta$-mercaptoethanol, $20 \mathrm{mM} \beta$-glycerophosphate, 10\% v:v glycerol,) using a Polytron homogenizer. The homogenates were then centrifuged at 13,500 $\times \mathrm{g}$ in an Eppendorf table-top centrifuge for 30 minutes at $4{ }^{\circ} \mathrm{C}$ and then the supernatant was removed.

A carboxymethyl $(\mathrm{CM})$ Sepharose cation exchange column $(10 \mathrm{~cm} \times 1 \mathrm{~cm})$ was prepared and equilibrated to $\mathrm{pH} 6.0$ by flowing $20 \mathrm{~mL}$ of buffer A through the column by gravity. The prepared muscle extract was then added to the top of the column and unbound protein was then eluted through gravity filtration by the addition of $15 \mathrm{~mL}$ of buffer A. Additional proteins were eluted from the column by increasing the $\mathrm{pH}$ through the addition of $15 \mathrm{~mL}$ of buffer $\mathrm{A}$ at $\mathrm{pH}$ 6.5. Finally, GDH was eluted from the $\mathrm{CM}$ column using $20 \mathrm{~mL}$ of buffer $\mathrm{A}$ at $\mathrm{pH} 7.0$. The fractions eluted from the $\mathrm{CM}$ column were assayed in the KG-consuming direction to detect GDH activity (see GDH Assay and Kinetics) and the top 5 fractions were pooled. The pooled fractions were then applied to a $1 \mathrm{~mL}$ column of $2^{\prime}, 5^{\prime}$-ADP agarose pre-equilibrated in $15 \mathrm{~mL}$ of buffer B (same components as buffer A but with $25 \mathrm{mM}$ imidazole buffer instead of MES). The column was then washed with the addition of $15 \mathrm{~mL}$ of buffer B to elute unbound protein and then a linear gradient of 0-5 mM NADP in buffer B was applied to the column over $30 \mathrm{~mL}$ to elute GDH. The fractions were then assayed as before and the top 8 fractions were pooled and applied to a GTP-agarose column pre-equilibrated in $15 \mathrm{~mL}$ of buffer $\mathrm{B}$. The protein sample was 
applied to the top of the column bed and allowed to filter through by gravity. Unbound protein was then eluted using $15 \mathrm{~mL}$ of buffer B. A linear $0-0.75 \mathrm{M} \mathrm{KCl}$ gradient in buffer B was then applied to the column and the resulting fractions were assayed to determine which ones contained GDH activity. The top 4 fractions were pooled and used for kinetic analysis of GDH.

\subsubsection{GDH Assay and Kinetics}

GDH activity was determined in both directions of the reaction. Assays in the KG consuming direction used $50 \mathrm{mM}$ imidazole buffer with standard assay conditions as follows: 0.3 mM NADH, $5 \mathrm{mM} \mathrm{KG,} 120 \mathrm{mM} \mathrm{NH}_{4}^{+}, 1 \mathrm{mM}$ ADP in a total reaction volume of $200 \mu \mathrm{l}$. Reactions were initiated by addition of $20 \mu \mathrm{l}$ of enzyme sample and the change in absorbance at $340 \mathrm{~nm}$ was monitored using a Thermo Multiskan ${ }^{\circledR}$ Spectrum microplate reader. Assays in the Glu consuming direction used the same assay buffer (50 $\mathrm{mM}$ imidazole) but at 8.0. The assay conditions were as follows: $40 \mathrm{mM}$ Glu, $4 \mathrm{mM}$ NAD and $1 \mathrm{mM}$ ADP with assays started by the addition of $30 \mu \mathrm{l}$ of enzyme and a total volume of $200 \mu \mathrm{l}$ in each microplate well. Unless otherwise stated, all assays were performed at $22^{\circ} \mathrm{C}$. For assays at $37^{\circ} \mathrm{C}$, the internal plate heater of the microplate reader was used. Assays performed at $8^{\circ} \mathrm{C}$ were accomplished by cooling the instrument in a low temperature incubator. The temperature of the wells was checked using a thermistor to ensure that they were at $8{ }^{\circ} \mathrm{C}$ before the addition of enzyme to begin the reaction. Studies of leucine activation of GDH were performed without the addition of ADP since no leucine activation was observed in the presence of optimal ADP concentrations.

Raw data collected from the microplate reader was analyzed using a microplate analysis program (MPA) (Brooks, 1994). One unit of GDH activity is defined as the amount of GDH required to convert $1 \mu \mathrm{mol}$ of NAD to NADH per minute under the conditions described above. 


\subsubsection{SDS-Polyacrylamide Gel Electrophoresis}

To determine the purity of the fractions, samples were prepared for sodium dodecyl sulfate polyacrylamide gel electrophoresis (SDS-PAGE) by mixing 1:1 v:v in SDS-PAGE loading buffer (100 mM Tris, pH 6.8, 4\% w/v SDS, $20 \% \mathrm{v} / \mathrm{v}$ glycerol, $0.2 \% \mathrm{w} / \mathrm{v}$ bromophenol blue) and boiling samples for 5 minutes to completely denature the proteins. Samples were then loaded onto $10 \%$ polyacrylamide gels and run at $180 \mathrm{~V}$ for 65 min using a BioRad Mini Protean III ${ }^{\circledR}$ apparatus. A lane containing 10.5-175 kDa PiNK Plus pre-stained protein ladder (FroggaBio \# PM005-0500) was run to confirm monomer sizes of ground squirrel proteins. Gels were stained using Coomassie Brilliant Blue stain until an even blue background was achieved followed by immersion of the gel in destain solution ( $18.5 \%$ methanol $\mathrm{v} / \mathrm{v}, 7.4 \% \mathrm{v} / \mathrm{v}$ acetic acid) until a strong contrast was achieved between the protein bands and the background.

\subsubsection{Western Blots}

Western blots were performed in order to confirm the identity of the GDH band and assess the protein levels in unpurified muscle lysates as well as to compare the relative levels of various potential post-translational modifications between the control and hibernating states. Purified GDH samples from euthermic and hibernating squirrels were first concentrated $\sim 26 \times$ using Amicon ${ }^{\circledR}$ Ultra-4 Centrifugal $10 \mathrm{kDa}$ concentrator units (Millipore-Sigma \#UFC801024) and then diluted appropriately to load equal amounts of protein across samples. Samples were then subjected to SDS-PAGE as described above. Following electrophoresis, proteins were transferred to an Immobilon-P polyvinylidene fluoride (PVDF) membrane (Millipore \# IPVH00010) pre-wetted with methanol using a BioRad Mini Trans-Blot ${ }^{\circledR}$ Electrophoretic Transfer Cell immersed in transfer buffer (25 mM Tris-base, $\mathrm{pH} 8.8,192 \mathrm{mM}$ glycine, 20\% v/v methanol) and set to $200 \mathrm{~mA}$ for 90 min with cooling on ice. Following transfer, blots used to 
assess the quantity of proteins in the crude tissue lysates were blocked using $2 \% \mathrm{v} / \mathrm{v}$ skim milk powder diluted in TBST (20 mM Tris base, $\mathrm{pH} 7.6,140 \mathrm{mM} \mathrm{NaCl}, 0.05 \% \mathrm{v} / \mathrm{v}$ Tween-20) for 30 min with gentle rocking. After blocking, the blots were washed in TBST with constant rocking for $3 \times 5$ minutes. Primary antibody (diluted 1:1000 in TBST) was then added and blots were incubated overnight at $4{ }^{\circ} \mathrm{C}$ in a refrigerator with constant rocking. Antibodies used were as follows:

1. Anti-phosphorylated serine rabbit IgG (Invitrogen \# 61-8100)

2. Anti-phosphorylated threonine rabbit IgG (Invitrogen \#71-8200)

3. Anti-phosphorylated tyrosine mouse IgG (Invitrogen \# 13-6600)

4. Anti-acetyl lysine rabbit IgG (Santa Cruz Biotechnology \# SC-8663)

5. Anti-succinyl lysine rabbit IgG (PTM Biolabs \# PTM-401)

6. Anti-glutamate dehydrogenase rabbit IgG (GeneTex \# GTX105765)

7. Anti-SIRT4 rabbit IgG (Santa Cruz Biotechnology \# SC-135053)

After incubation with the primary anti-body, blots were washed $3 \times 5$ minutes in TBST to remove unbound antibody. Membranes were then incubated with rocking in anti-IgG antibody conjugated to horseradish peroxidase (diluted 1:8000 in TBST) raised against either rabbit or mouse IgG, as appropriate. Detection was carried using an enhanced chemiluminesence (ECL) protocol and imaged with a ChemiGenius Bioimaging System (Syngene, Frederick, MD). After a picture was developed with strong contrast, the blots were stained using Coomassie brilliant blue and pictures were taken using the same instrument with internal lighting activated. For blots quantifying the relative levels of GDH and SIRT4 in tissue lysates, a group of stably expressed 
proteins on the Coomassie stained membranes were used as loading controls (Eaton et al., 2013). The relative levels of PTMs in purified GDH were adjusted to the amount GDH detected in the Coomassie stained blots. Analysis of the blots was performed using GeneTools 4.3.8.0 software.

\subsubsection{Software and Statistics}

Enzyme activity data was analyzed using a Microplate Analysis Program (MPA) (Brooks, 1994). Enzyme kinetic parameters were calculated using a nonlinear least squares regression data modelling program, Kinetics 3.5.1, following the Hill equation (Brooks, 1992). Data was considered statistically different between euthermic and hibernating when $\mathrm{p}<0.05$ by a two-tailed Student's t-test. When comparing multiple parameters, as was the case when comparing $K_{m}$ data across temperature for either experimental group, an ANOVA test was performed with Tukey's post-hoc test. Statistical tests were performed in the RBioplot program environment (Zhang \& Storey, 2016). Graphs were also constructed using the RBioplot program.

\subsubsection{In Silico ADP-Ribosylation Prediction}

Since Richardson's ground squirrel is not sequenced, the GDH protein sequence for the closely related North American 13-lined ground squirrel (Ictidomys tridecemlineatus), was used for bioinformatics analyses (NCBI accession number: XP_021587637.1). The online ADPredict ADP-ribosylation prediction tool was utilized to search for putative aspartate and glutamate residues within the ground squirrel GDH sequence that might be subject to this PTM (Monte, Manelfi, Gemei, Corda, \& Beccari, 2018). Residues that were predicted (prediction score greater than 0.4) to be ADP-ribosylated were highlighted across a conserved domain map of the GDH sequence (Lu et al., 2020). 


\subsection{Results}

\subsubsection{Purification}

Purification of skeletal muscle GDH followed a three-step column chromatography procedure (Table 5.1.). The first step utilized a cation exchange column that bound proteins at $\mathrm{pH}$ 6.0. Buffer $\mathrm{pH}$ was then raised to $\mathrm{pH}$ 6.5, which eluted many other proteins while GDH remained bound. A further increase to $\mathrm{pH} 7.0$ then eluted GDH. This step resulted in a 65\% yield and an 8.8 fold increase in specific activity of GDH. The next step made use of a 2',5'-ADP agarose column that bound proteins based on their ability to recognize NADP. The column was eluted using a 0-5 mM NADP gradient that increased the fold purification to $111 \times$ relative to the crude homogenate. In the last step, GDH was bound to a GTP-agarose affinity chromatography column and eluted by increasing $\mathrm{KCl}$ concentration. This step yielded a final fold purification of $340 \times$ as compared to the crude specific activity. The final specific activity was $\sim 2680 \mathrm{mU} / \mathrm{mg}$ (Table 5.1.). Visualizing the purification steps using SDS-PAGE with Coomassie staining demonstrated very low amounts of protein (below detectable levels) after the ADP-agarose step, so the final sample after the GTP-agarose step was concentrated $10 \times$. The $10 \times$ concentrated sample showed a single band corresponding with the bovine hepatic GDH standard (Fig. 5.1.). Identity of this band as GDH was further confirmed through a western blot performed using crude lysates with an anti-GDH antibody that revealed a GDH band in this same location (Appendix C.1.).

\subsubsection{GDH Kinetics}

In the Glu consuming direction, several differences were noted in the affinities for substrates, activators, and the inhibitor GTP between muscle GDH from euthermic and hibernating conditions. The $K_{m}$ Glu was the same for the purified enzymes from both conditions 
when assessed at 22 and $37^{\circ} \mathrm{C}$ (Fig. 5.2. A). However, this pattern did not hold true at $8{ }^{\circ} \mathrm{C}$ where it was noted that the euthermic enzyme had significantly higher (2.1-fold) $K_{m}$ for Glu at this temperature than did the hibernating form (Fig. 5.2. A). This was due to a substantial increase in the $K_{m}$ at this temperature for euthermic GDH that did not occur for the hibernating enzyme. For $\mathrm{NAD}^{+}$substrate, higher temperatures were associated with higher $K_{m}$ values for both euthermic and hibernating GDH, although this was only notable for the euthermic value at $37^{\circ} \mathrm{C}$, whereas in the hibernating situation the increase in the $K_{m}$ occurred at a lower temperature, $22{ }^{\circ} \mathrm{C}$, and actually decreased slightly at $37^{\circ} \mathrm{C}$ (Fig. 5.2. B). The effects of the activator, ADP, on GDH activity in the Glu-consuming direction were quite similar between the euthermic and hibernating enzyme, although a slight decrease in the fold-activation by ADP ( $V_{\max }$ ratio at $1 \mathrm{mM}$ vs $0 \mathrm{mM}$ ADP) was noted for the hibernating enzyme relative to the euthermic form when assayed at $37^{\circ} \mathrm{C}$ (Table 5.2.). The effects of leucine as an activator were assessed only at $22{ }^{\circ} \mathrm{C}$ and neither $K_{A}$ values nor $V_{\max }$ ratio for leucine (10 $\mathrm{mM} / 0 \mathrm{mM}$ Leucine) differed between the euthermic and hibernating enzyme. GTP is a known inhibitor of GDH and the $I_{50}$ value for GTP/Mg $\mathrm{Mg}^{2+}\left(1: 2 \mathrm{mM} \mathrm{GTP} / \mathrm{Mg}^{2+}\right)$ was significantly higher (by 1.25 fold) for the hibernating form of GDH, compared with the euthermic control (Table 5.2.). At $22{ }^{\circ} \mathrm{C}$, no significant difference was observed in the GDH maximal activity in the Glu consuming direction (Table 5.2.).

In the $\alpha-K G$ consuming direction, no statistically significant differences were noted between the euthermic and the hibernating conditions for either the $K_{m} \alpha$-ketoglutarate (Fig. 5.3. A) or the maximal activity $\left(V_{\max }, \mathrm{mU} / \mathrm{mg}\right)$ at $22^{\circ} \mathrm{C}$ (Table 5.2.). Additionally, no changes were noted in terms of how temperature affected $K_{m} \alpha$-ketoglutarate for either the euthermic or hibernating purified enzyme (Fig. 5.3. A). This was in contrast to the $K_{m} \mathrm{NH}_{4}{ }^{+}$that was notably 
lower at every temperature for the euthermic enzyme compared with hibernator GDH (Fig. 5.3. B). $K_{m}$ values for $\mathrm{NH}_{4}{ }^{+}$were not dependent on temperature over the range tested in these experiments $\left(8-37^{\circ} \mathrm{C}\right)$ for either the euthermic or hibernating enzyme forms (Fig. 5.3. B). However, the effects of ADP activation seemed to depend on both temperature and experimental group (Table 5.2.). Euthermic GDH showed a significantly higher $K_{A} \mathrm{ADP}$ at $8{ }^{\circ} \mathrm{C}$ and a higher fold increase in GDH activity ( $V_{\max }$ ratio at $1 \mathrm{mM}$ vs $0 \mathrm{mM}$ ADP) in the presence of ADP relative to the hibernating enzyme. However, assays at $22{ }^{\circ} \mathrm{C}$ showed no significant differences between euthermic and hibernating GDH for either $K_{A} \mathrm{ADP}$ or the $V_{\max }$ ratio. The situation changed again at $37^{\circ} \mathrm{C}$ with the fold activation owing to maximal ADP activation being higher for the hibernating enzyme than for the euthermic condition. Interestingly, a significant decrease in the $K_{A}$ for leucine was noted in the hibernating group ( 0.53 -fold) in assays at $22{ }^{\circ} \mathrm{C}$, although no change in the $V_{\max }$ ratio for leucine $(10 \mathrm{mM} / 0 \mathrm{mM})$ was noted for this activator (Table 5.2.).

\subsubsection{Activities in Crude Preparations}

The activity of GDH was determined in both directions of the reaction under optimal substrate concentrations at $22{ }^{\circ} \mathrm{C}$. No significant difference was noted in the maximal activity standardized per mg of protein in the muscle tissue lysates from euthermic and hibernating ground squirrels (Fig. 5.4.).

\subsubsection{Quantification of GDH and SIRT4}

No difference was found in the quantification of GDH determined by western blot analysis of total protein homogenates using a GDH antibody (Fig. 5.5.). Similarly, no changes were found in the levels of SIRT4, an important mitochondrial enzyme known to have a role in regulating GDH activity through post-translational modification by ADP-ribosylation. 


\subsubsection{Western Blot Analysis of PTMs}

Western blots were used to assess the relative levels of various PTMs on purified ground squirrel GDH, comparing euthermic and hibernating states. These blots showed very few differences in the levels of various PTMs commonly associated with enzymatic regulation. Notable, however, was an increase in the relative level of ADP-ribosylation (1.48-fold increase) on the hibernating form of the enzyme, as compared with euthermic GDH (Fig. 5.6.).

\subsubsection{In Silico ADP-Ribosylation Prediction}

Use of bioinformatics resources revealed 7 sites that were predicted to be potential ADPribosylation sites on glutamate residues within the I. tridecemlineatus sequence (see Appendix C.2.). All of these residues were found in the NAD binding domain, several of which were located in close proximity to specific residues that are associated with directly binding NAD. For example, E165 (highlighted in red) was found to be both predicted as an ADP-ribosylation site and is actively involved in NAD binding (Fig. 5.7.).

\subsection{Discussion}

Hibernators go through extended periods of metabolic reshaping during the winter to reduce their energy expenditure and allowing them to survive fasting throughout the entire season. During hibernation, animals utilize stored lipid reserves to maintain a greatly reduced metabolic rate and avoid using other fuel supplies. GDH plays a critical central regulatory role in the mitochondria by mediating the reaction that bridges amino acid catabolism and the CAC. Glutamate produced through protein breakdown and the catabolism of various amino acids can be metabolized in the mitochondria to produce $\alpha$-ketoglutarate $(\alpha-K G)$ and NADH. The $\alpha-K G$ can be subsequently oxidized two more times in the CAC to generate $2 \mathrm{NADH}$ molecules and $\mathrm{FADH}_{2}$ that in turn can be used in oxidative phosphorylation via the electron transport chain in 
addition to the NADH that generated through oxidative deamination by GDH. Because of its capacity to control the flow of intermediate substrates into the CAC, GDH's role in reduced metabolic states has been investigated in a variety of organisms. Generally protein and amino acid catabolism is thought to be of minimal significance in fueling muscle metabolism since carbohydrates and lipids tend to be preferred, but during intense exercise it is estimated that amino acid catabolism may produce about 5-10\% of the muscle energy needs (Poortmans, 1984).

The results from the current study show a complex pattern of regulation of ground squirrel GDH. No changes were observed in GDH specific activity in the crude muscle lysates between the euthermic and hibernating samples and this was matched by the total relative amount of GDH remaining constant as demonstrated by immunoblotting. Similarly, no changes were noted in $V_{\max }$ of either the forward or reverse directions for the purified enzyme samples. In spite of this, several notable differences were observed in the affinity of the purified enzyme for its substrates and activators/inhibitors. The most consistently observed difference across the three assay temperatures was the decrease in affinity for $\mathrm{NH}_{4}{ }^{+}$seen in the hibernating enzyme samples. The physiological significance of this change is not easily understood since the $K_{m}$ for this substrate is far higher than the expected $\mathrm{NH}_{4}{ }^{+}$concentrations in muscle tissue and as such, the magnitude of this effect in vivo may not be readily experienced in the animal. For example, the concentration of ammonium ion in muscle tissue is typically only around $\sim 1 \mathrm{mmol} / \mathrm{kg}$ dry weight, although this can be increased substantially during vigorous exercise and the mitochondrial concentrations may differ from the tissue concentrations as a whole (Graham \& MacLean, 1992). Nonetheless, the stable differences in affinity for ammonium ion suggest that there exists some mechanism of regulation on the level of the protein itself that alters its functional properties during the hibernation period. In the other direction of the reaction, the Glu 
consuming one favored in vivo, a notable difference was observed at $8{ }^{\circ} \mathrm{C}$ in the $K_{m}$ Glu between the euthermic and hibernating forms of the enzyme, with the euthermic parameter being over 2fold greater than the hibernating, despite the value being consistent between the other temperatures assayed. This seems somewhat odd that GDH from hibernating animals has a greater affinity for Glu than in euthermia, but it should be noted that this is not so much an increase in affinity since the affinity for Glu actually remained relatively constant across temperature for the hibernating enzyme, but rather the euthermic $K_{m}$ greatly increased (Fig 5.2.). Conversely, both euthermic and hibernating forms of the enzyme seem to have a greater affinity for $\mathrm{NAD}^{+}$at $8{ }^{\circ} \mathrm{C}$ as compared to normal body temperature $\left(37^{\circ} \mathrm{C}\right)$. The hibernating enzyme maintained a higher affinity for $\mathrm{NAD}^{+}$at 8 and $37^{\circ} \mathrm{C}$, although this trend was reversed at $22{ }^{\circ} \mathrm{C}$. This might suggest that during the deepest state of torpor, that the GDH enzyme may actually be modified in order to maintain sufficient activity during the coldest periods of hibernation during the winter months when body temperatures can drop to near freezing. A reduction in the sensitivity to GTP inhibition on the enzyme by the hibernating form may also support that this conclusion.

The maintenance of GDH activity in the catabolic direction during hibernation seems somewhat at odds with the general trend towards energy sparing. The lack of muscle wasting and maintenance of proteins in the muscle also would seem to suggest that levels of free amino acids available for incorporation into the $\mathrm{CAC}$ would be reduced during this time as well. The plasma levels of several amino acids including Glu and aspartate are known to increase during the late arousal phase and then subsequently drop down during the interbout arousal phase, potentially suggesting that there may be some increased catabolism of proteins during this period (Buck \& Barnes, 1999; Regan et al., 2019). The reason for this increase in amino acid catabolism has been 
suggested as means of facilitating gluconeogenesis to replenish blood glucose levels rather than as a fuel source (Buck \& Barnes, 2000). However, this may only be true in the liver since gluconeogenesis tends to occur at higher rates in this tissue. Muscle-wasting during hibernation is avoided despite the sedentary nature of the hibernator so perhaps the significance of the decrease in GTP inhibition and higher Glu affinity at lower temperatures of hibernating GDH lies in the increased plasma Glu levels during the arousal periods (Bodine, 2013; Goropashnaya, Barnes, \& Fedorov, 2020; Xu et al., 2013). The elevated levels of amino acids could be used in the muscle to help provide added fuel for rewarming as the animal shivers to increase body temperature. The maintenance of GDH activity in the Glu consuming direction may therefore be important to ensure sufficient activity to process the increase in plasma Glu during early and late arousal phases as a substrate to channel into the CAC.

The alterations in some of the kinetic parameters of GDH suggested that changes in the post-translational modifications of the enzyme might occur in the hibernation group to affect changes in amino acid catabolism and CAC function. Investigation of the relative levels of several common PTMs demonstrated that most of the PTMs examined remained at relatively constant levels. However, the levels of ADP-ribosylation were almost $1.5 \times$ higher in hibernation than in euthermia. ADP-ribosylation takes two general forms in nature: mono-ADP-ribosylation (MARylation), which is less well-characterized, and poly-ADP-ribosylation (PARylation), which is commonly associated with DNA repair mechanisms (Liu, Vyas, Kassab, Singh, \& Yu, 2017). In PARylation, chains of poly-ADP-ribose as long as 200 units may be linked to an amino acid side chain (typically lysine or an acidic residue) (Gagné, Shah, \& Poirier, 2001). While the ADPribose binding reagent used for probing the western blots is not capable of distinguishing between the mono and poly-ADP-ribosyl groups, PARylation is highly unlikely in the context of 
these results since the addition of such large moieties would significantly reduce the electrophoretic mobility of the GDH protein. MARylation on a cysteine residue is known to be a regulatory mechanism of GDH and has an inhibitory effect on GDH (Choi et al., 2005; Haigis et al., 2006; Kim, Yang, Choi, Lee, \& Cho, 2012). This canonical sort of MARylation was suspected in the case of wood frog hepatic GDH that was reduced in maximal activity during whole body freezing (Green \& Storey, 2017). The significance of the ADP-ribosylation observed here is not entirely clear owing to the lack of the $V_{\max }$ inhibition that is typically associated with GDH MARylation and it could be that the ADP-ribosylation in this case may occur at a different, non-canonical location on GDH. The use of bioinformatics tools demonstrated several putative sites for glutamate ADP-ribosylation on ground squirrel GDH. One of these was located directly in the $\mathrm{NAD}^{+}$binding site but seems an unlikely candidate for the PTM responsible for the changes here since this would likely completely deactivate the enzyme. Several other ADPribosylation sites occur further away from the active site residues and may be responsible for the changes observed in $\mathrm{NAD}^{+}$binding kinetics and potentially the other changes observed here. However, numerous other types of residues besides the acidic residues examined using this bioinformatics resource are known to be ADP-ribosylated and could potentially account for the differences in ADP-ribosylation observed here including lysine, cysteine, arginine, serine and asparagine (Laing, Koch-Nolte, Haag, \& Buck, 2011; Leidecker et al., 2016; McDonald \& Moss, 1994). Some of these ADP-ribosylations have strong associations with specific subcellular compartments, such as serine and lysine ADP-ribosylation which is best known to be associated with histones and usually takes the form of poly-ADP-ribosylation and in this case are therefore unlikely candidates (Bonfiglio et al., 2017; Messner et al., 2010). 
Mitochondrial sirtuin 4 (SIRT4) is known to carry out MARylation on GDH to inhibit its activity (Ahuja et al., 2007). For this reason, the relative levels of SIRT4 were examined in muscle tissue samples from euthermic and hibernating squirrels using immunoblotting but no differences were observed between the two groups. In another species, the 13-lined ground squirrel, SIRT4 levels also remained consistent between euthermic muscle samples and samples taken at varying points during the torpor-arousal cycle (Rouble \& Storey, 2015). Because the SIRT4 levels are constant between the euthermic and hibernating states, it is likely that another mechanism is responsible for the differences observed in GDH ADP-ribosylation between the euthermic and hibernating animals. This is perhaps expected since while GDH is a known target for MARylation by SIRT4, as discussed earlier it seems unlikely that the ADP-ribosylation takes place on the previously described residue owing to the lack of reduction in $V_{\max }$ in the more highly ADP-ribosylated form. An increase in the levels of ADP-ribosylation was also noted in the E1 subunit of the $\alpha$-ketoglutarate dehydrogenase complex of the Richardson's ground squirrel during torpor (Green \& Storey, 2020). Taken together with the increase in ADPribosylation on GDH observed here, this suggests that there may be a common mechanism aiding regulation of these two mitochondrial enzymes that are linked in both the synthesis and catabolism of glutamate and amino acids derived from glutamate. Additionally, SIRT4 is not known to have broad-spectrum ADP-ribosyl transferase (ART) activity but instead has so far only been reported to target GDH (Hopp \& Hottiger, 2021). At least two other ADP-ribosyl transferases have been described in mitochondria. Mitochondrial ADP-ribosyl transferase 1 (ART1) is primarily thought to catalyze MARylation as opposed to PARylation (Bonfiglio et al., 2020; Hopp \& Hottiger, 2021). Recently it has been reported that another ART, Neuralized-like 4 (NEURL4), may actually be responsible for the majority of ADP-ribosylation in the 
mitochondria, although it is primarily thought to be PARylation. There is also the possibility that the availability of $\mathrm{NAD}^{+}$needed for ART activity may be altered during hibernation (Haigis et al., 2006; Hopp \& Hottiger, 2021). While the relative ratio of $\mathrm{NAD}^{+} / \mathrm{NADH}$ is not known for muscle mitochondria of hibernating versus euthermic animals, it has been suggested that perturbations in the balance of reduced and oxidized forms of these cofactors likely favors NAD ${ }^{+}$ over NADH during hibernation (Klug \& Brigham, 2015). Perturbations in the level of $\mathrm{NAD}^{+}$in the mitochondria could potentially explain the increase in ADP-ribosylation observed in this present study and in the ketoglutarate dehydrogenase E1 subunit that has been reported previously during torpor (Green \& Storey, 2020).

The regulation of GDH has been assessed previously during states of metabolic depression owing to its critical role in bridging amino acid metabolism and the CAC. Most relevant to the current study were other studies on U. richardsonii that compared the properties of hepatic GDH during hibernation versus euthermia. In the liver a decrease in the hibernating GDH $K_{m}$ Glu was noted at $22{ }^{\circ} \mathrm{C}$ and $5{ }^{\circ} \mathrm{C}$, although this was reversed at $37{ }^{\circ} \mathrm{C}$ where the $K_{m}$ was higher in the hibernating (Bell \& Storey, 2010; Thatcher \& Storey, 2001). This was in contrast to the results observed in the current study that noted a greater euthermic glutamate $K_{m}$ only at 8 ${ }^{\circ} \mathrm{C}$. While there are some similarities in the present study of muscle GDH to observations for liver GDH, notably the greater Glu affinity at low temperatures for the hibernating enzyme, there are also marked differences since the $K_{m}$ values were stable across a wide variety of temperatures in the hibernating in the muscle tissue. Liver GDH from ground squirrels showed decreased total protein phosphorylation in the hibernating form and these differences seemed to be related to $K_{m}$ when endogenous kinase activity was stimulated (Bell \& Storey, 2010). By comparison, no difference in the overall phosphorylation level was observed on any of the 
commonly phosphorylated amino acids of muscle GDH and this may help to explain the differences between the kinetic parameters between the two tissues. Differences in isoform expression between the two tissues is unlikely since, although humans and other apes have two

isoforms of GDH, the ground squirrel is only known to have one isoform (Pajęcka et al., 2014). The role of GDH regulation in other animals that utilize metabolic rate depression is better understood for liver GDH than the muscle form. However, muscle GDH of two invertebrates that undergo metabolic rate depression has been examined. In the foot muscle of the terrestrial snail (Otala lactea), GDH activity during estivation was observed to be increased by approximately 3fold relative to the control group, suggesting that amino acid catabolism has greater importance under these conditions (Bell, Dawson, \& Storey, 2012). This contrasted with results for a crayfish species (Orconectes virilis) where severe hypoxia was associated with increased $K_{m}$ Glu (Dawson \& Storey, 2012). While these models are vastly different from the hibernating squirrel it is interesting to note that GDH is a common target for metabolic regulation in muscle tissue in a wide variety of animals, reflecting the need to modulate the amino acid oxidizing capacity.

\subsection{Conclusion}

The data presented here shows modest changes to the functional and structural characteristics of GDH in the muscle tissue of hibernating Richardson's ground squirrels relative to euthermic controls. This suggests a certain degree of metabolic fine-tuning that may help to maintain GDH activity to some degree despite the strongly reduced body temperatures of the hibernating state through maintenance of a stable affinity for Glu, as opposed to the euthermic state where the substrate affinity decreased at $8{ }^{\circ} \mathrm{C}$. Decreased inhibition due to GTP may also contribute to GDH control during hibernation and the PTM status of GDH suggests that ADPribosylation is higher on hibernating GDH. This PTM is likely different from the canonical GDH 
ADP-ribosylation of cysteine residues and likely takes place at a different location due to the lack of an observed decrease in $V_{\max }$ of the hibernating enzyme that would normally be expected. These findings add to a previous study implicating mono-ADP-ribosylation as a potential tool in the regulation of central metabolic enzymes within the mitochondria during ground squirrel hibernation. 
Table 5.1. Representative purification of GDH from skeletal muscle of hibernating Richardson's ground squirrel

\begin{tabular}{|c|c|c|c|c|c|}
\hline Step & $\begin{array}{l}\text { Total } \\
\text { Protein } \\
(\mathrm{mg})\end{array}$ & $\begin{array}{l}\text { Total Activity } \\
\text { (KG } \\
\text { consuming) } \\
(\mathrm{mU})\end{array}$ & $\begin{array}{l}\text { \% Yield from } \\
\text { Crude }\end{array}$ & $\begin{array}{l}\text { Fold } \\
\text { Purification } \\
\text { from Crude }\end{array}$ & $\begin{array}{l}\text { Specific } \\
\text { Activity } \\
(\mathrm{mU} / \mathrm{mg})\end{array}$ \\
\hline Crude & 108 & 850 & NA & $\mathrm{NA}$ & 7.9 \\
\hline $\mathrm{CM}(\mathrm{pH} 7.0)$ & 7.9 & 551 & 65 & 8.8 & 69 \\
\hline $\begin{array}{l}2^{\prime}, 5^{\prime}-A D P \\
\text { Agarose (0-5 } \\
\text { mM NADP) }\end{array}$ & 0.33 & 289 & 34 & 111 & 870 \\
\hline $\begin{array}{l}\text { GTP }(0-0.75 \\
\text { M KCl })\end{array}$ & 0.07 & 198 & 23 & 340 & 2678 \\
\hline
\end{tabular}


Table 5.2. Comparing the potencies of activators and inhibitors of GDH at three different temperatures between euthermic control and hibernating Richardson's ground squirrel GDH at 3 different temperatures. Data represent the mean $\pm \mathrm{SEM}, n=4$.

Glutamate consuming

\begin{tabular}{|c|c|c|}
\hline Parameter & Euthermic & Hibernating \\
\hline \multicolumn{3}{|l|}{$8^{\circ} \mathrm{C}$} \\
\hline $\mathrm{K}_{\mathrm{A}} \mathrm{ADP}(\mathrm{mM})$ & $0.033 \pm 0.003$ & $0.034 \pm 0.008$ \\
\hline $\mathrm{V}_{\max }$ Ratio (1 mM ADP/ 0 mM ADP) & $1.57 \pm 0.02$ & $1.53 \pm 0.09$ \\
\hline \multicolumn{3}{|l|}{$22^{\circ} \mathrm{C}$} \\
\hline $\mathrm{V}_{\max }(\mathrm{mU} / \mathrm{mg})$ & $505 \pm 17$ & $543 \pm 3.8$ \\
\hline $\mathrm{K}_{\mathrm{A}} \mathrm{ADP}(\mathrm{mM})$ & $0.034 \pm 0.003$ & $0.026 \pm 0.003$ \\
\hline $\mathrm{V}_{\max }$ Ratio (1 mM ADP/ 0 mM ADP) & $1.89 \pm 0.04$ & $1.81 \pm 0.02$ \\
\hline $\mathrm{K}_{\mathrm{A}}$ Leucine $(\mathrm{mM})$ & $1.1 \pm 0.2$ & $1.2 \pm 0.1$ \\
\hline $\mathrm{V}_{\max }$ Ratio Leucine (10 mM Leucine / $0 \mathrm{mM}$ ) & $1.68 \pm 0.06$ & $1.84 \pm 0.09$ \\
\hline $\mathrm{I}_{50} \mathrm{GTP} / \mathrm{Mg}^{2+}(1: 2 \mathrm{mM})$ & $0.0109 \pm 0.0001$ & $0.0136 \pm 0.0006^{*}$ \\
\hline \multicolumn{3}{|l|}{$37^{\circ} \mathrm{C}$} \\
\hline $\mathrm{K}_{\mathrm{A}} \mathrm{ADP}(\mathrm{mM})$ & $0.0266 \pm 0.0007$ & $0.029 \pm 0.003$ \\
\hline $\mathrm{V}_{\max }$ Ratio (1 mM ADP/ 0 mM ADP) & $2.21 \pm 0.05$ & $1.93 \pm 0.08^{*}$ \\
\hline \multicolumn{3}{|l|}{$\alpha-K G$ consuming } \\
\hline Parameter & Euthermic & Hibernating \\
\hline \multicolumn{3}{|l|}{$8^{\circ} \mathrm{C}$} \\
\hline $\mathrm{K}_{\mathrm{A}} \operatorname{ADP}(\mathrm{mM})$ & $0.052 \pm 0.004$ & $0.032 \pm 0.004^{*}$ \\
\hline $\mathrm{V}_{\max }$ ratio $(1 \mathrm{mM} \mathrm{ADP} / 0 \mathrm{mM} \mathrm{ADP})$ & $1.60 \pm 0.04$ & $1.21 \pm 0.06^{*}$ \\
\hline \multicolumn{3}{|l|}{$22^{\circ} \mathrm{C}$} \\
\hline $\mathrm{V}_{\max }(\mathrm{mU} / \mathrm{mg})$ & $2299 \pm 75$ & $2482 \pm 117$ \\
\hline $\mathrm{K}_{\mathrm{A}} \mathrm{ADP}(\mathrm{mM})$ & $0.035 \pm 0.004$ & $0.051 \pm 0.006$ \\
\hline $\mathrm{V}_{\max }$ Ratio (1 mM ADP/ 0mM ADP) & $1.87 \pm 0.08$ & $1.64 \pm 0.1$ \\
\hline $\mathrm{K}_{\mathrm{A}}$ Leucine $(\mathrm{mM})$ & $0.55 \pm 0.05$ & $0.29 \pm 0.03^{*}$ \\
\hline $\mathrm{V}_{\max }$ Ratio Leucine $(10 \mathrm{mM} / 0 \mathrm{mM}$ & $2.2 \pm 0.2$ & $1.91 \pm 0.05$ \\
\hline \multicolumn{3}{|l|}{$37^{\circ} \mathrm{C}$} \\
\hline $\mathrm{K}_{\mathrm{A}} \mathrm{ADP}(\mathrm{mM})$ & $0.053 \pm 0.004$ & $0.070 \pm 0.007$ \\
\hline $\mathrm{V}_{\max }$ Ratio (1 mM ADP/ 0 mM ADP) & $1.80 \pm 0.07$ & $2.2 \pm 0.1^{*}$ \\
\hline
\end{tabular}




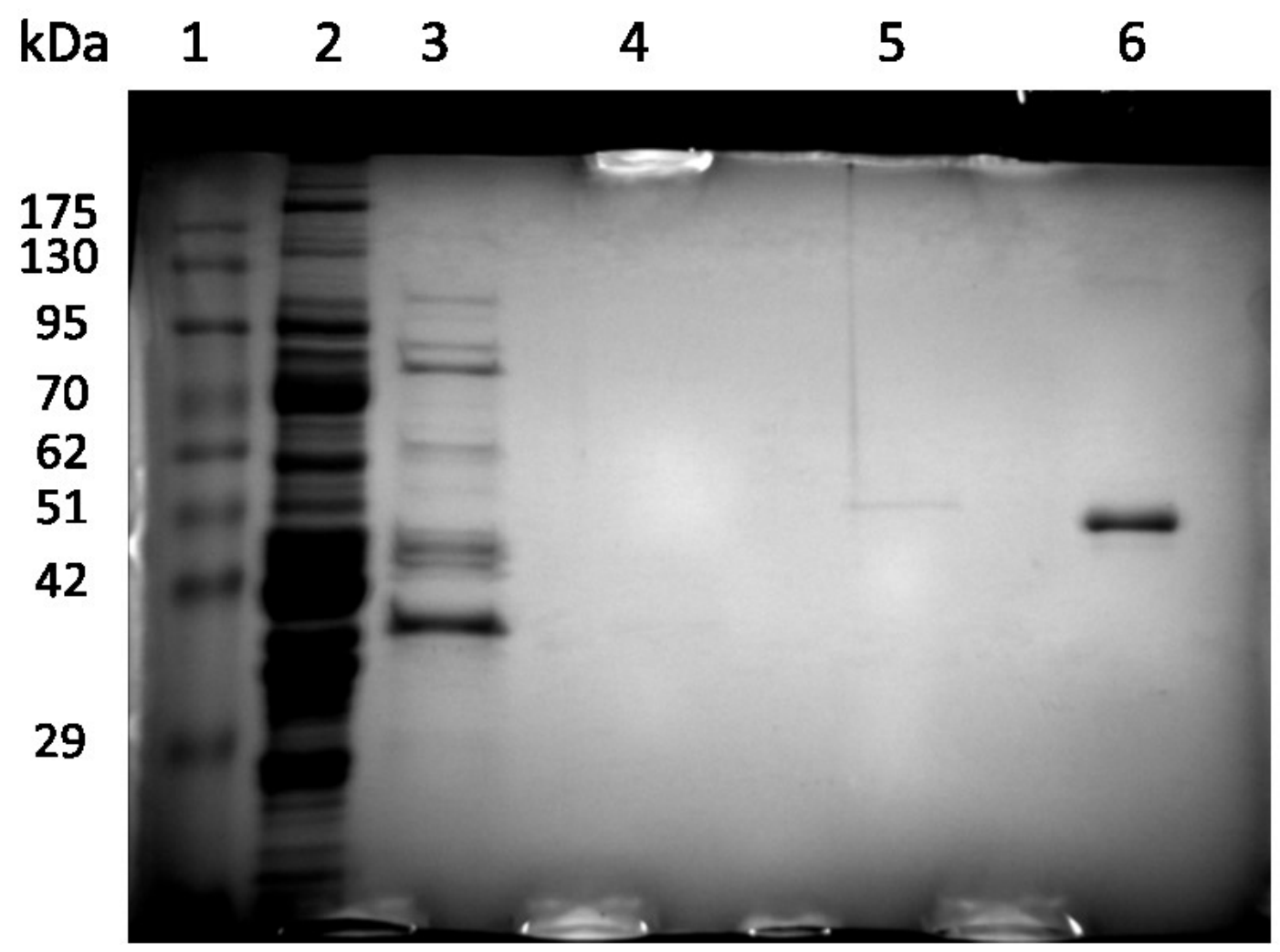

Fig. 5.1. Coomassie stained SDS-PAGE gel showing purification of GDH from muscle of euthermic ground squirrels. Lanes are as follows: (1) Pre-stained protein ladder (GeneDirex 10.5-175 kDa), (2) Crude muscle protein extract, (3) Elution from CM column by increasing $\mathrm{pH}$, (4) Eluate from peak fractions of 2',5'-ADP agarose following 0-5 mM NADP gradient, (5) $10 \times$ concentrated GTP-agarose eluate following elution with 0-0.75 M KCl gradient, (6) Commercial hepatic bovine GDH. 

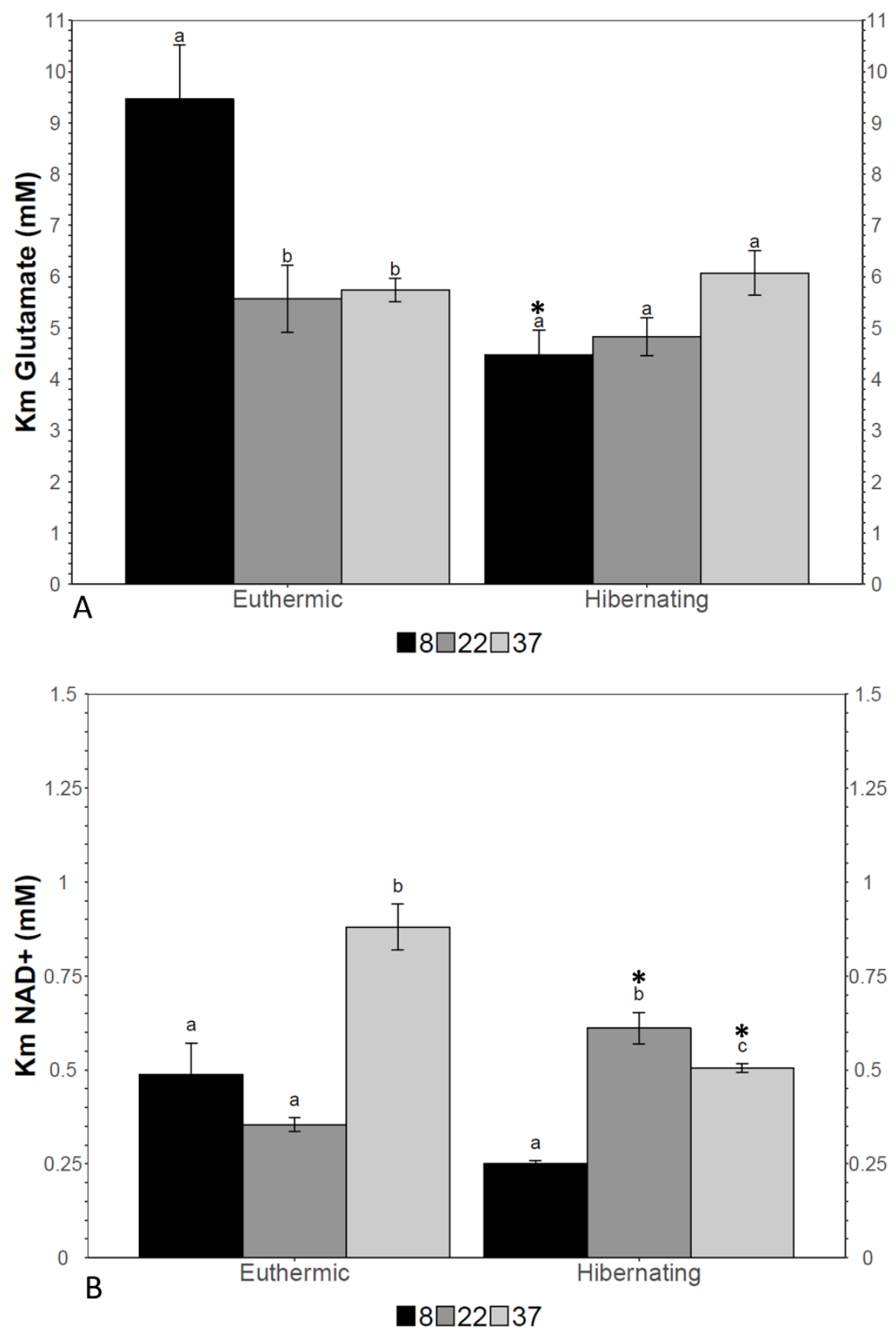

Fig. 5.2. $K_{m}$ values of GDH substrates in the glutamate consuming (forward direction) of the reaction at three different temperatures $\left(8,22,37^{\circ} \mathrm{C}\right)$ performed on purified GDH from the skeletal muscle of Richardson's ground squirrel (Urocitellus richardsonii). Data are mean \pm $\mathrm{SEM}, \mathrm{n}=4$. Letters above the bars show results of analysis of variance test with a post hoc Tukey's test; different letters within one experimental group represent significant changes between temperatures $(\mathrm{p}<0.05)$. ' $*$ ' indicates that the hibernating value is significantly different from the corresponding control euthermic value at the same temperature, according to a twotailed Student's $t$-test $(\mathrm{p}<0.05)$. A) $K_{m}$ for glutamate, B) $K_{m}$ for NAD ${ }^{+}$ 

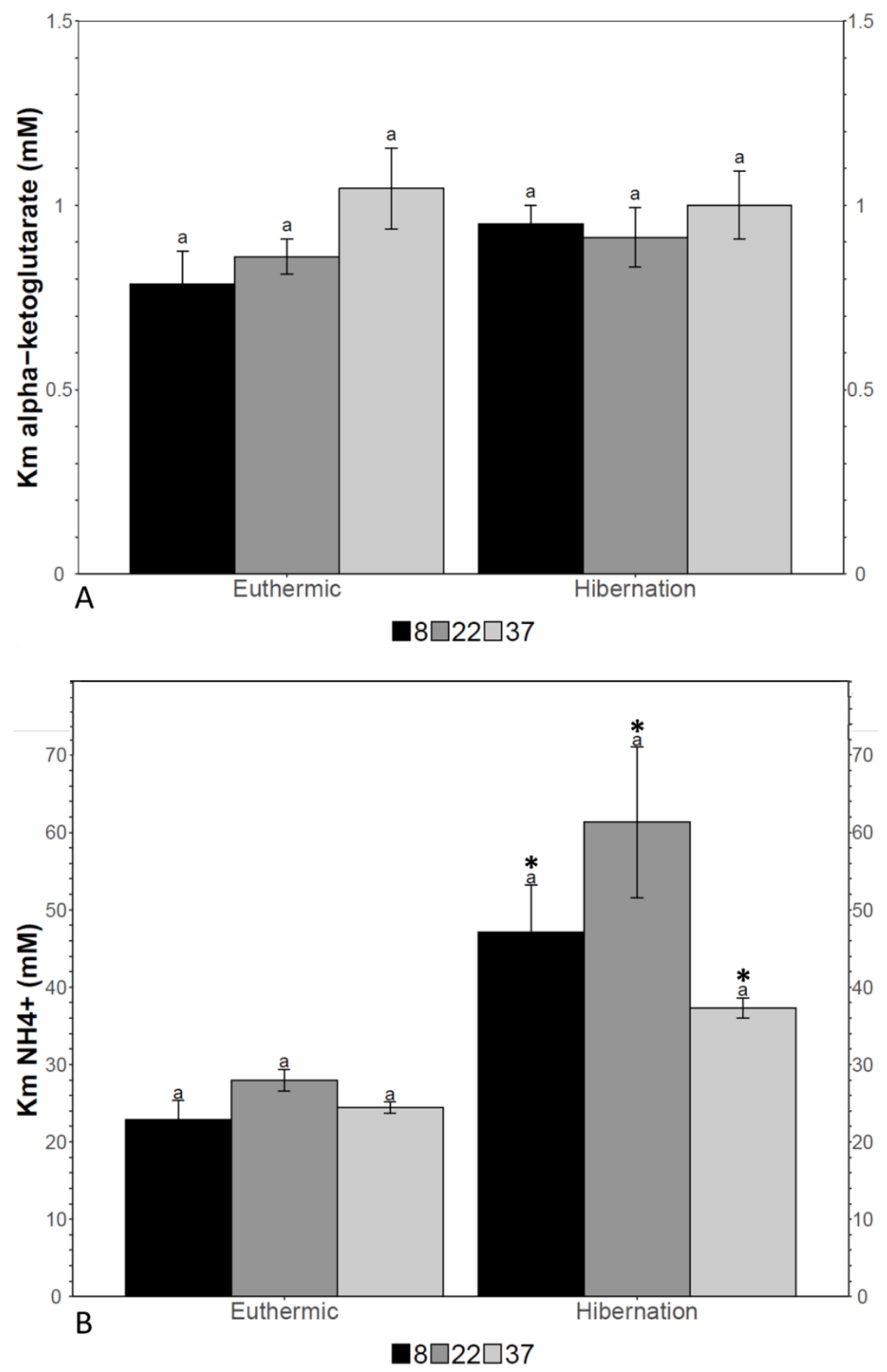

Fig. 5.3. $\mathrm{Km}$ values for GDH substrates in the glutamate producing (reverse direction) of the reaction at three different temperatures $\left(8,22,37^{\circ} \mathrm{C}\right)$ performed on purified GDH from the skeletal muscle of Richardson's ground squirrel (Urocitellus richardsonii). A) $K_{m}$ for $\alpha-$ ketoglutarate, B) $K_{m}$ for $\mathrm{NH}_{4}{ }^{+}$. All other information is the same as in Fig. 5.2. 


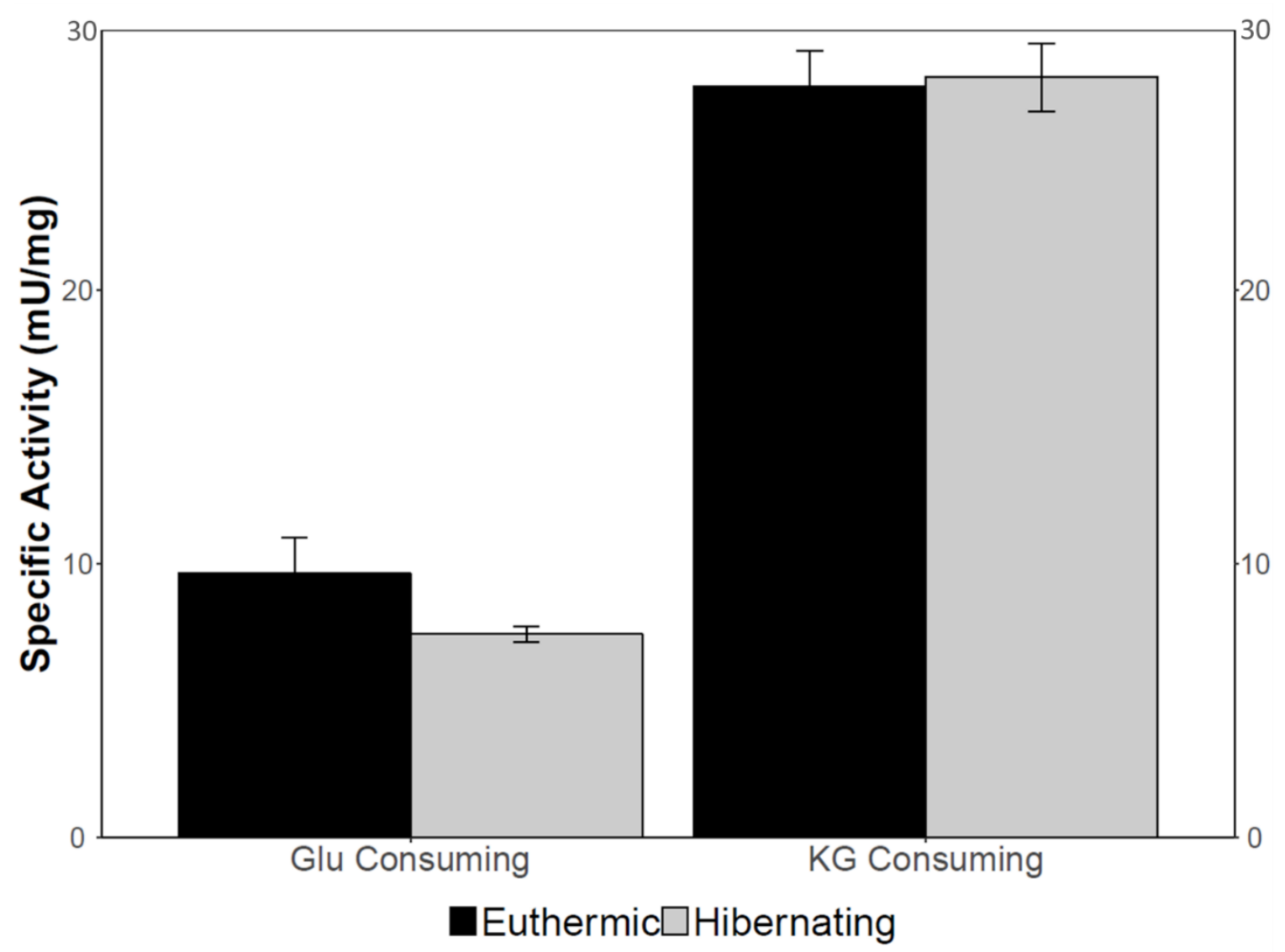

Fig. 5.4. Demonstrating the maximum specific activity of GDH in the glutamate-consuming direction glutamate-producing directions in euthermic and hibernating Richardson's ground squirrel muscle tissues lysates at $22{ }^{\circ} \mathrm{C}$. Data are means $\pm \mathrm{SEM}, \mathrm{n}=4$. 


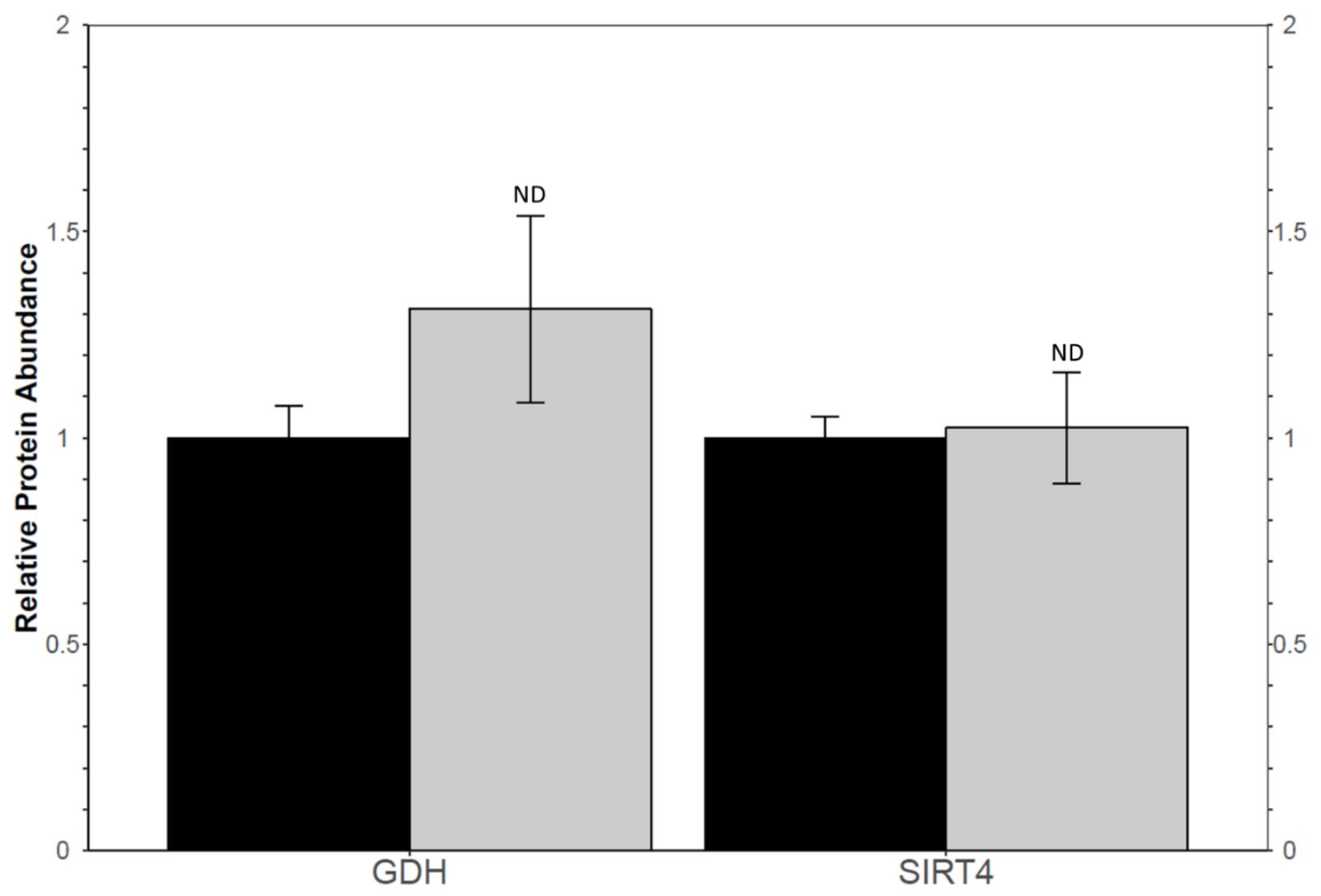

Euthermic $\square$ Hibernating
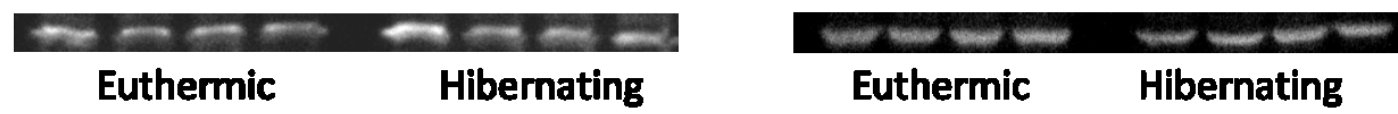

Fig. 5.5. Quantification of GDH and SIRT4 levels in skeletal muscle lysates from euthermic and hibernating Richardson's ground squirrel. The GDH bands are depicted below the graph with the four left-most bands being the euthermic samples and the four on the right being the hibernating samples. A two-tailed Student's t-test was applied to the data and demonstrated no significant difference (ND) between the euthermic and hibernating samples $(\mathrm{p}<0.05)$. Data are mean \pm $\mathrm{SEM}, \mathrm{n}=4$ samples from different preparations. 


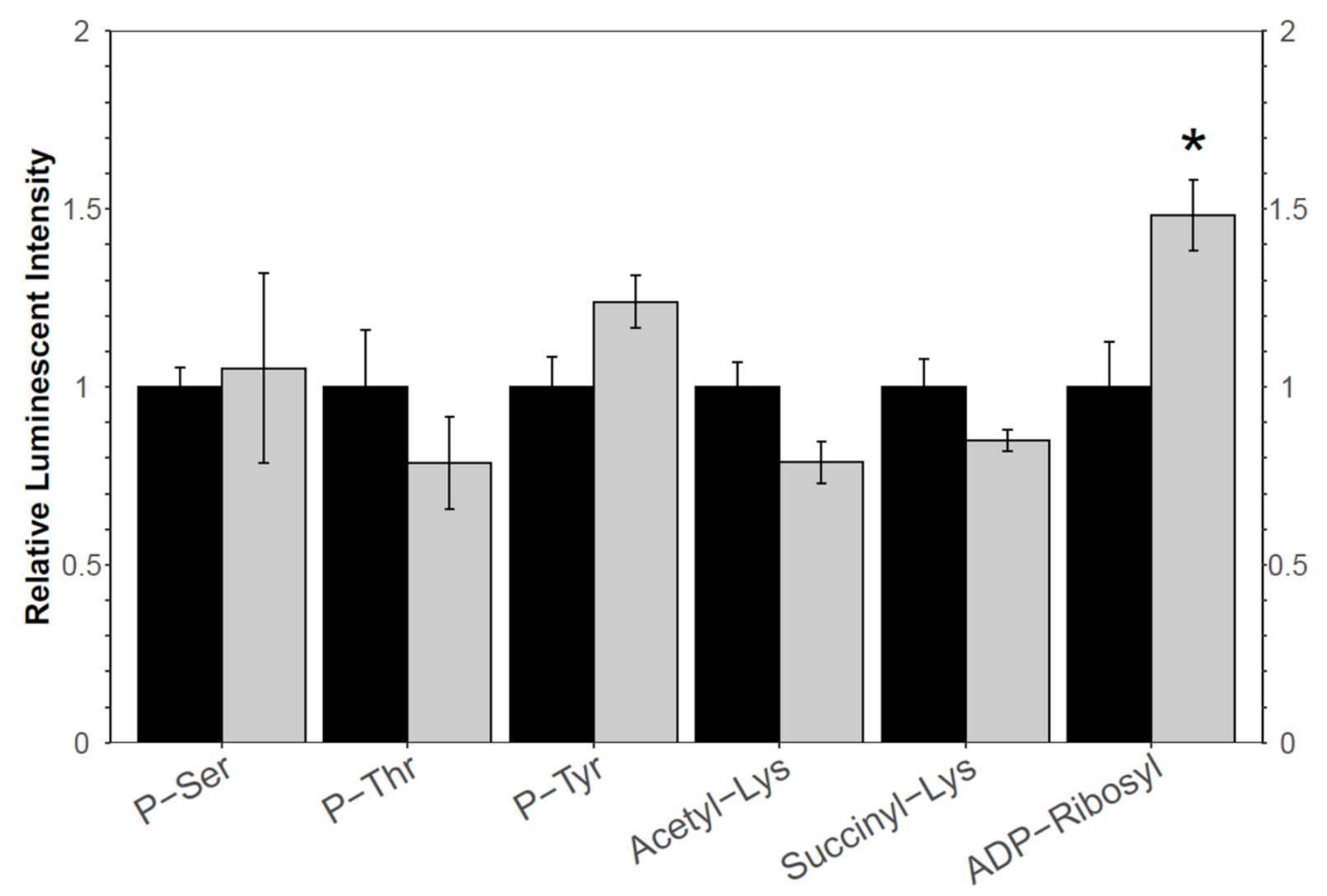

Euthermic $\square$ Hibernating

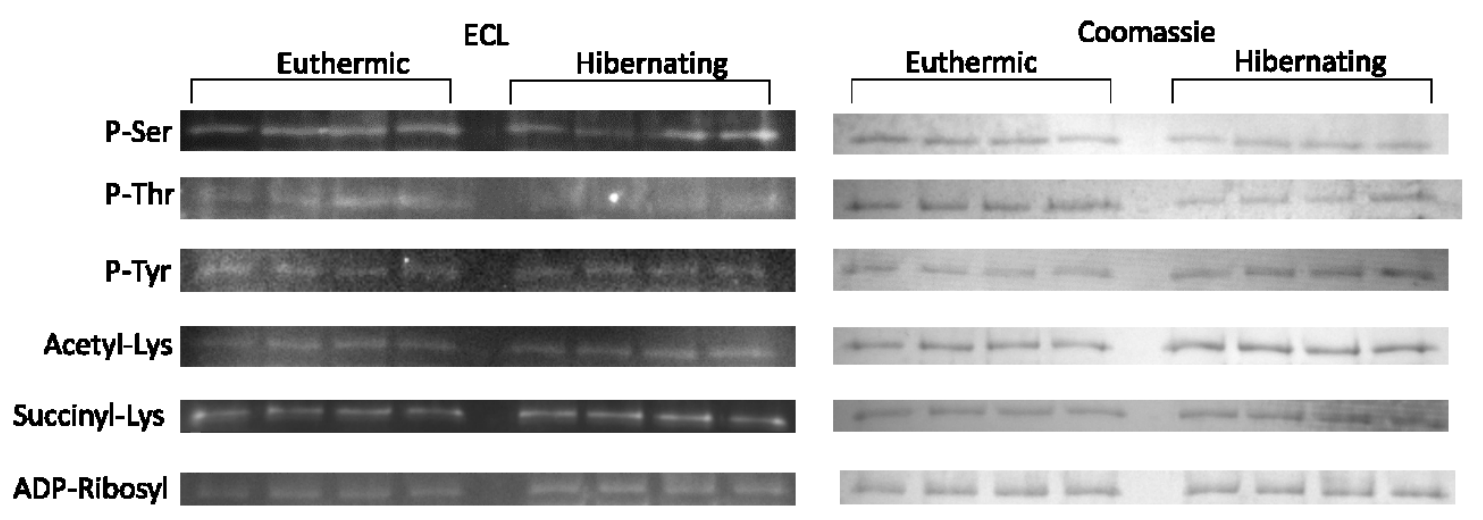

Fig 5.6. Western blots of purified Richardson's ground squirrel skeletal muscle GDH samples demonstrating the relative changes in various post-translational modifications. The bar chart shows the enhanced chemiluminescent (ECL) signal adjusted for the protein amount in each band as determined by Coomassie staining with the euthermic mean value adjusted to 1 and the hibernating value presented relative to the euthermic. Data are mean $\pm \mathrm{SEM}, \mathrm{n}=4$; ' $*$ ' indicates that the hibernating parameter is statistically different from the euthermic value according to Student's t-test, $\mathrm{p}<0.05$. 


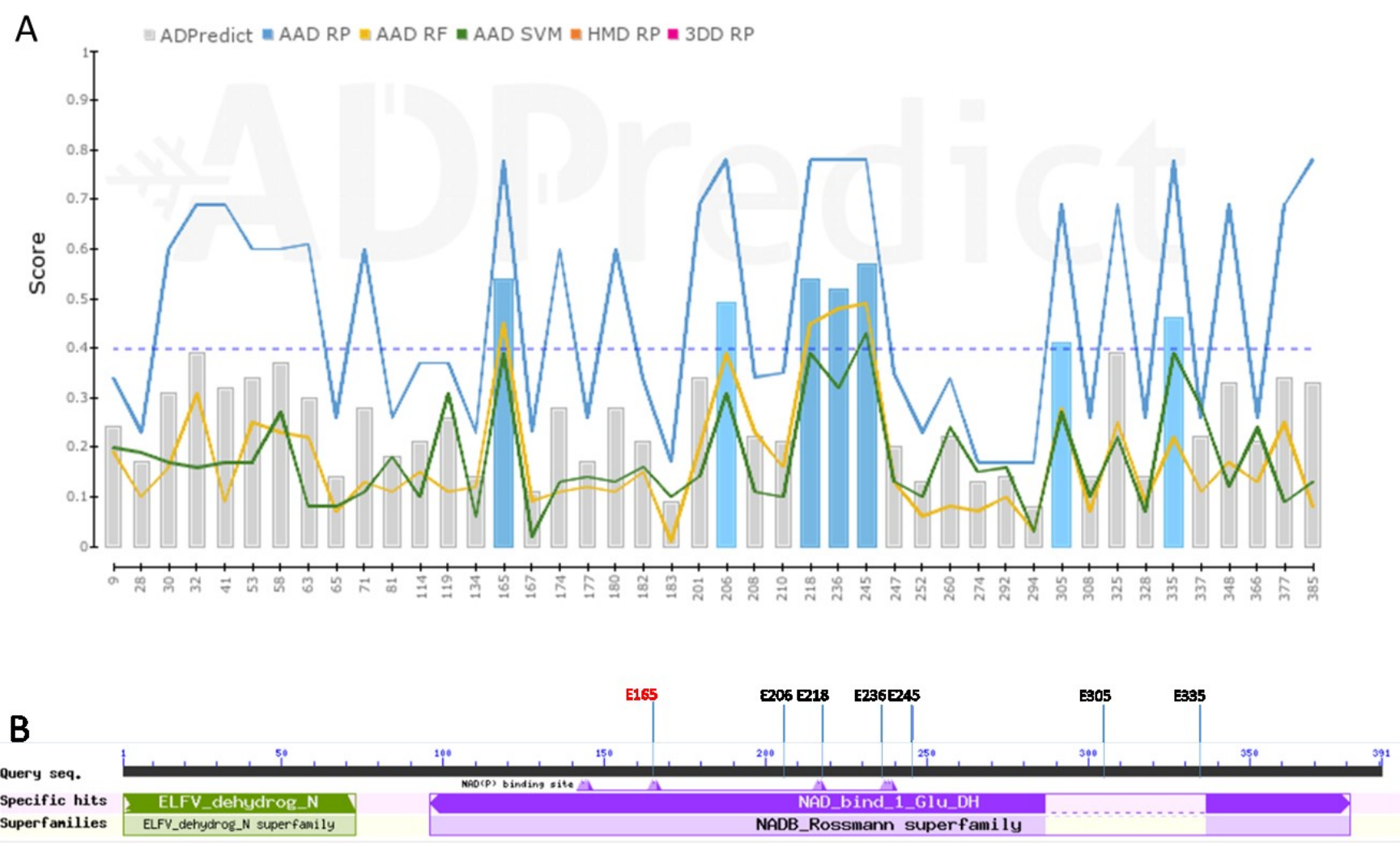

Fig. 5.7. A. ADP-ribosylation prediction of I. tridecemlineatus glutamate dehydrogenase using ADPredict bioinformatics resource. Y-axis demonstrates the prediction score for glutamate and aspartate residues (threshold for prediction is 0.4 ). The prediction score is the average of three different prediction algorithms based on amino acid descriptors (AAD); 1. Recursive Partitioning Tree (RP), 2. Random Forests (RF), 3. Support Vector Machines (SVM). Other modeling algorithms (HMD and 3DD RP) required a solved structure and were not used. B. Predicted ADP-ribosyl sites are overlapped against a conserved domain map of the GDH sequence. The residue in red is an ADP-ribosylated predicted site that is involved in NAD binding. 


\section{Chapter 5 References}

Ahuja, N., Schwer, B., Carobbio, S., Waltregny, D., North, B. J., Castronovo, V., ... Verdin, E. (2007). Regulation of insulin secretion by SIRT4, a mitochondrial ADP-ribosyltransferase. Journal of Biological Chemistry, 282(46), 33583-33592. https://doi.org/10.1074/jbc.M705488200

Aleshin, V. A., Mkrtchyan, G. V., Kaehne, T., Graf, A. V., Maslova, M. V., \& Bunik, V. I. (2020). Diurnal regulation of the function of the rat brain glutamate dehydrogenase by acetylation and its dependence on thiamine administration. Journal of Neurochemistry, 153(1), 80-102. https://doi.org/10.1111/jnc.14951

Bell, R. A. V, \& Storey, K. B. (2010). Regulation of liver glutamate dehydrogenase by reversible phosphorylation in a hibernating mammal. Comparative Biochemistry and Physiology - B Biochemistry and Molecular Biology, 157(3), 310-316. https://doi.org/10.1016/j.cbpb.2010.07.005

Bell, R. A. V., Dawson, N. J., \& Storey, K. B. (2012). Insights into the in vivo regulation of glutamate dehydrogenase from the foot muscle of an estivating land snail. Enzyme Research, 2012. https://doi.org/10.1155/2012/317314

Bodine, S. C. (2013). Hibernation: The search for treatments to prevent disuse-induced skeletal muscle atrophy. Experimental Neurology, 248, 129-135. https://doi.org/10.1016/j.expneurol.2013.06.003

Bonfiglio, J. J., Fontana, P., Zhang, Q., Colby, T., Gibbs-Seymour, I., Atanassov, I., ... Matic, I. (2017). Serine ADP-Ribosylation Depends on HPF1. Molecular Cell, 65(5), 932-940.e6. https://doi.org/10.1016/j.molcel.2017.01.003

Bonfiglio, J. J., Leidecker, O., Dauben, H., Longarini, E. J., Colby, T., San Segundo-Acosta, P., ... Matic, I. (2020). An HPF1/PARP1-Based Chemical Biology Strategy for Exploring ADP-Ribosylation. Cell, 183(4), 1086-1102.e23. https://doi.org/10.1016/j.cell.2020.09.055

Brooks, S. P. (1994). A program for analyzing enzyme rate data obtained from a microplate reader. Biotechniques, 17(6), 1154-1161. Retrieved from http://www.ncbi.nlm.nih.gov/entrez/query.fcgi?cmd=Retrieve\&db=PubMed\&dopt=Citation \&list_uids $=7873186$

Brooks, S. P. J. (1992). A simple computer program with statistical tests for the analysis of enzyme kinetics. BioTechniques, 13(6), 906-911. Retrieved from http://www.ncbi.nlm.nih.gov/pubmed/1476744

Brosnan, J. T. (2000). Glutamate, at the interface between amino acid and carbohydrate metabolism. The Journal of Nutrition, 130(4S Suppl), 988S-90S. 
Buck, C. L., \& Barnes, B. M. (1999). Annual cycle of body composition and hibernation in freeliving arctic ground squirrels. Journal of Mammalogy, 80(2), 430-442. https://doi.org/10.2307/1383291

Buck, C. L., \& Barnes, B. M. (2000). Effects of ambient temperature on metabolic rate, respiratory quotient, and torpor in an arctic hibernator. American Journal of Physiology Regulatory Integrative and Comparative Physiology, 279, R255-R262. https://doi.org/10.1152/ajpregu.2000.279.1.r255

Chinopoulos, C. (2013). Which way does the citric acid cycle turn during hypoxia? The critical role of $\alpha$-ketoglutarate dehydrogenase complex. Journal of Neuroscience Research, 91(8), 1030-1043. https://doi.org/10.1002/jnr.23196

Choi, M. M., Huh, J. W., Yang, S. J., Eun, H. C., Soo, Y. C., \& Cho, S. W. (2005). Identification of ADP-ribosylation site in human glutamate dehydrogenase isozymes. FEBS Letters, 579, 4125-4130. https://doi.org/10.1016/j.febslet.2005.06.041

Dawson, N. J., \& Storey, K. B. (2012). An enzymatic bridge between carbohydrate and amino acid metabolism: Regulation of glutamate dehydrogenase by reversible phosphorylation in a severe hypoxia-tolerant crayfish. Journal of Comparative Physiology B: Biochemical, Systemic, and Environmental Physiology, 182(3), 331-340. https://doi.org/10.1007/s00360011-0629-4

Eaton, S. L., Roche, S. L., Llavero Hurtado, M., Oldknow, K. J., Farquharson, C., Gillingwater, T. H., \& Wishart, T. M. (2013). Total protein analysis as a reliable loading control for quantitative fluorescent western blotting. PLoS ONE, 8(8), e72457. https://doi.org/10.1371/journal.pone.0072457

Gagné, J. P., Shah, R. G., \& Poirier, G. G. (2001). Analysis of ADP-ribose polymer sizes in intact cells. Molecular and Cellular Biochemistry, 224(1-2), 183-185. https://doi.org/10.1023/A:1011910329010

Goropashnaya, A. V., Barnes, B. M., \& Fedorov, V. B. (2020). Transcriptional changes in muscle of hibernating arctic ground squirrels (Urocitellus parryii): implications for attenuation of disuse muscle atrophy. Scientific Reports. https://doi.org/10.1038/s41598020-66030-9

Graham, T. E., \& MacLean, D. A. (1992). Ammonia and amino acid metabolism in human skeletal muscle during exercise. Canadian Journal of Physiology and Pharmacology, 70, 132-141. https://doi.org/10.1139/y92-020

Green, S. R., \& Storey, K. B. (2017). Regulation of glutamate dehydrogenase (GDH) in response to whole body freezing in wood frog liver linked to differential acetylation and ADPribosylation. Archives of Biochemistry and Biophysics, 636, 90-99. https://doi.org/10.1016/j.abb.2017.10.010 
Green, S. R., \& Storey, K. B. (2020). Regulation of the $\alpha$-ketoglutarate dehydrogenase complex during hibernation in a small mammal, the Richardson's ground squirrel (Urocitellus richardsonii). Biochimica et Biophysica Acta - Proteins and Proteomics, 1868(9). https://doi.org/10.1016/j.bbapap.2020.140448

Green, S. R., \& Storey, K. B. (2021). Functional and post-translational characterization of pyruvate dehydrogenase demonstrates repression of activity in the liver but not skeletal muscle of the Richardson's ground squirrel (Urocitellus richardsonii) during hibernation. Journal of Thermal Biology. https://doi.org/10.1016/j.jtherbio.2021.102996

Hadj-Moussa, H., Green, S. R., \& Storey, K. B. (2018). The living dead: mitochondria and metabolic arrest. IUBMB Life, 70(2), 1260-1266. https://doi.org/10.1002/iub.1910

Haigis, M. C., Mostoslavsky, R., Haigis, K. M., Fahie, K., Christodoulou, D. C., Murphy, A. J., ... Guarente, L. (2006). SIRT4 inhibits glutamate dehydrogenase and opposes the effects of calorie restriction in pancreatic b cells. Cell, 126(5), 941-954. https://doi.org/10.1016/j.cell.2006.06.057

Herrero-Yraola, A., Bakhit, S. M. A., Franke, P., Weise, C., Schweiger, M., Jorcke, D., \& Ziegler, M. (2001). Regulation of glutamate dehydrogenase by reversible ADP-ribosylation in mitochondria. EMBO Journal, 20(10), 2404-2412. https://doi.org/10.1093/emboj/20.10.2404

Hopp, A. K., \& Hottiger, M. O. (2021). Uncovering the invisible: mono-ADP-ribosylation moved into the spotlight. Cells, 10(3). https://doi.org/10.3390/cells10030680

Kim, E. A., Yang, S. J., Choi, S. Y., Lee, W. J., \& Cho, S. W. (2012). Inhibition of glutamate dehydrogenase and insulin secretion by KHG26377 does not involve ADP-ribosylation by SIRT4 or deacetylation by SIRT3. BMB Reports, 45(8), 458-463. https://doi.org/10.5483/BMBRep.2012.45.8.040

Klug, B. J., \& Brigham, R. M. (2015). Changes to metabolism and cell physiology that enable mammalian hibernation. Springer Science Reviews, 3(1), 39-56. https://doi.org/10.1007/s40362-015-0030-x

Laing, S., Koch-Nolte, F., Haag, F., \& Buck, F. (2011). Strategies for the identification of arginine ADP-ribosylation sites. Journal of Proteomics, 75(1), 169-176. https://doi.org/10.1016/j.jprot.2011.07.003

Leidecker, O., Bonfiglio, J. J., Colby, T., Zhang, Q., Atanassov, I., Zaja, R., ... Matic, I. (2016). Serine is a new target residue for endogenous ADP-ribosylation on histones. Nature Chemical Biology, 12(12), 998-1000. https://doi.org/10.1038/nchembio.2180

Liu, C., Vyas, A., Kassab, M. A., Singh, A. K., \& Yu, X. (2017). The role of poly ADPribosylation in the first wave of DNA damage response. Nucleic Acids Research, 45(14), 8129-8141. https://doi.org/10.1093/nar/gkx565 
Lu, S., Wang, J., Chitsaz, F., Derbyshire, M. K., Geer, R. C., Gonzales, N. R., ... MarchlerBauer, A. (2020). CDD/SPARCLE: The conserved domain database in 2020. Nucleic Acids Research, 48(D1), D265-D268. https://doi.org/10.1093/nar/gkz991

Małecki, J., Jakobsson, M. E., Ho, A. Y. Y., Moen, A., Rustan, A. C., \& Falnes, P. (2017). Uncovering human METTL12 as a mitochondrial methyltransferase that modulates citrate synthase activity through metabolite-sensitive lysine methylation. Journal of Biological Chemistry, 292(43), 17950-17962. https://doi.org/10.1074/jbc.M117.808451

McDonald, L. J., \& Moss, J. (1994). Enzymatic and nonenzymatic ADP-ribosylation of cysteine. Molecular and Cellular Biochemistry, 138(1-2), 221-226. https://doi.org/10.1007/BF00928465

Messner, S., Altmeyer, M., Zhao, H., Pozivil, A., Roschitzki, B., Gehrig, P., ... Hottiger, M. O. (2010). PARP1 ADP-ribosylates lysine residues of the core histone tails. Nucleic Acids Research, 38(19), 6350-6362. https://doi.org/10.1093/nar/gkq463

Michener, G. R. (1998). Sexual differences in reproductive effort of Richardson's ground squirrels. Journal of Mammalogy, 79(1), 1-19. https://doi.org/10.2307/1382838

Monte, M. Lo, Manelfi, C., Gemei, M., Corda, D., \& Beccari, A. R. (2018). ADPredict: ADPribosylation site prediction based on physicochemical and structural descriptors. Bioinformatics, 34(15), 2566-2574. https://doi.org/10.1093/bioinformatics/bty159

Pajęcka, K., Nielsen, C. W., Hauge, A., Zaganas, I., Bak, L. K., Schousboe, A., ... Waagepetersen, H. S. (2014). Glutamate dehydrogenase isoforms with N-terminal (His)6or FLAG-tag retain their kinetic properties and cellular localization. Neurochemical Research. https://doi.org/10.1007/s11064-013-1042-z

Palaiologos, G., \& Felig, P. (1978). Influence of L-leucine on glutamate dehydrogenase activity in isolated rat diaphragm. Yale Journal of Biology and Medicine, 51(1), 19-25.

Poortmans, J. R. (1984). Protein turnover and amino acid oxidation during and after exercise. Medicine and Sport Science, 17, 130-147. https://doi.org/10.1159/000408783

Regan, M. D., Chiang, E., Martin, S. L., Porter, W. P., Assadi-Porter, F. M., \& Carey, H. V. (2019). Shifts in metabolic fuel use coincide with maximal rates of ventilation and body surface rewarming in an arousing hibernator. American Journal of Physiology - Regulatory Integrative and Comparative Physiology, 316(6), R764-R775. https://doi.org/10.1152/ajpregu.00379.2018

Rhein, V. F., Carroll, J., Ding, S., Fearnley, I. M., \& Walker, J. E. (2017). Human METTL12 is a mitochondrial methyltransferase that modifies citrate synthase. FEBS Letters, 591(12), 1641-1652. https://doi.org/10.1002/1873-3468.12649

Rouble, A. N., \& Storey, K. B. (2015). Characterization of the SIRT family of NAD+-dependent protein deacetylases in the context of a mammalian model of hibernation, the thirteen-lined 
ground squirrel. Cryobiology, 71(2), 334-343.

https://doi.org/10.1016/j.cryobiol.2015.08.009

Ruderman, N. B. (1975). Muscle amino acid metabolism and gluconeogenesis. Annual Review of Medicine, 26, 245-258. https://doi.org/10.1146/annurev.me.26.020175.001333

Spanaki, C., \& Plaitakis, A. (2012). The role of glutamate dehydrogenase in mammalian ammonia metabolism. Neurotoxicity Research, 21(1), 117-127.

https://doi.org/10.1007/s12640-011-9285-4

Thatcher, B. J., \& Storey, K. B. (2001). Glutamate dehydrogenase from liver of euthermic and hibernating Richardson's ground squirrels: evidence for two distinct enzyme forms. Biochemistry and Cell Biology = Biochimie et Biologie Cellulaire, 79(1), 11-19. https://doi.org/10.1139/o00-086

Wang, L. C. H. (1979). Time patterns and metabolic rates of natural torpor in the Richardson's ground squirrel. Canadian Journal of Zoology, 57(2), 149-155. https://doi.org/10.1139/z79012

Wang, L. C. H., \& Lee, T. F. (1996). Torpor and hibernation in mammals: Metabolic, physiological, and biochemical adaptations. Handbook of Physiology - Environmental Physiology, 507-532. https://doi.org/10.1002/cphy.cp040122

Xu, R., Andres-Mateos, E., Mejias, R., MacDonald, E. M., Leinwand, L. A., Merriman, D. K., ... Cohn, R. D. (2013). Hibernating squirrel muscle activates the endurance exercise pathway despite prolonged immobilization. Experimental Neurology, 247, 392-401. https://doi.org/10.1016/j.expneurol.2013.01.005

Zhang, J., \& Storey, K. B. (2016). RBioplot: an easy-to-use R pipeline for automated statistical analysis and data visualization in molecular biology and biochemistry. PeerJ, 4, e2436. https://doi.org/10.7717/peerj.2436

Zhou, X., \& Thompson, J. R. (1996). Regulation of glutamate dehydrogenase by branched-chain amino acids in skeletal muscle from rats and chicks. International Journal of Biochemistry and Cell Biology, 28(7), 787-793. https://doi.org/10.1016/1357-2725(96)00019-2 


\section{Chapter 6: General Discussion}




\subsection{General Discussion}

Seasonal hibernators undergo yearly cycles that involve complex alterations of their body composition, metabolism and physiological processes. In the case of the Richardson's ground squirrel, most of the year may be spent in torpor (up to 9 months in some cases), meaning that they may spend only enough time in the active euthermic state to mate and gain sufficient fat supplies to enter into the next torpor cycle (Wang, 1979). To this end, the animals engage in hyperphagia over the summer months to rapidly acquire these large fat reserves in adipose tissue to support the next hibernation season (Dark, 2005; Mrosovsky, 1974). Triglycerides are the preferred medium for fuel storage in mammals and most terrestrial vertebrates because of their high energy density relative to carbohydrates $(38 \mathrm{~kJ} / \mathrm{g}$ vs $17 \mathrm{~kJ} / \mathrm{g})$ and hydrophobic nature that allows them to be stored without excessive amounts of water bulk (Berg, Tymoczko, \& Stryer, 2002). For this reason, it should come as no surprise that hibernating animals greatly suppress their glycolytic functions in order to preferentially consume the lipids that were accumulated over the summer and stored into white and brown adipose tissue (Florant, 1998; Lanaspa et al., 2015). In at least one hibernating species (Ursus thibetanus japonincus), an increase in expression of several genes involved in $\beta$-oxidation of fats is present to facilitate the increased reliance on lipid catabolism during hibernation as well as a decrease in gene products necessary for lipogenesis in the liver (Shimozuru, Kamine, \& Tsubota, 2012). While much research has investigated the role of glycolytic suppression in the ground squirrel and other hibernators (Abnous \& Storey, 2008; Brooks \& Storey, 1992; Chazarin et al., 2019; Smolinski, Green, \& Storey, 2020), little to date has looked at the regulation of the CAC and other peripheral pathways feeding into this critical central metabolic hub during hibernation. The degree of regulation over the $\mathrm{CAC}$ is not as immediately obvious as the carbohydrate consuming pathways 
since animals still need to run this pathway in order to maintain a basal level of energy production for vital processes. However, it seems likely that the CAC pathway would be restricted to spare fat supplies from being used unnecessarily. This thesis examined the regulation of crucial CAC enzymes and also those that are external to the CAC but bridge the entry of fuels from glycolytic and amino acid catabolic pathways into the CAC in the muscle of the Richardson's ground squirrel.

The reliance on fatty acid oxidation during hibernation is well-known and has been demonstrated by a clear shift in the respiratory quotient during torpor from 1 to $0.7-0.8$ (Buck \& Barnes, 2000). Nonetheless, in skeletal muscle tissue of hibernators, some glycolysis still occurs, albeit at a much lower rate than during euthermia ( $\sim 10 \%$ of the total energy output) (Galster \& Morrison, 1970; Tashima, Adelstein, \& Lyman, 1970). This is evidenced by the fact that pyruvate and lactate are still generated during torpor, although at a slower rate (Galster \& Morrison, 1975). The maintenance of a limited flux through glycolysis in the muscle tissue may in part explain the lack of alterations in the activity of PDH or its phosphorylation state, as demonstrated in Chapter 2 (Green \& Storey, 2021). While PDH was not directly affected, a decrease in the activity of upstream glycolytic enzymes during hibernation would help to reduce the rate at which pyruvate is generated, indirectly limiting the activity of PDH. The fact that glycolysis still occurs at a limited rate in the muscle tissue would mean that PDH activity could safely be maintained during torpor, as long as the upstream glycolytic enzyme activity is restricted, without wasting precious carbohydrates. This is in contrast to what was seen in the liver where a substantial decrease in the activity and increase in the phosphorylation of a key inhibitory residue on PDH was observed. This difference may be important during torpor, since gluconeogenesis is thought to occur in the liver to maintain blood glucose levels especially 
during inter-bout arousals (Galster \& Morrison, 1975). Indeed gluconeogenic activity has been observed to be increased by up to 2-fold in cold acclimated summer and torpid squirrels relative to non-acclimated animals in hepatocytes of a another ground squirrel species (Staples \& Hochachka, 1998). The block in PDH activity in the liver would prevent futile-cycling, promote gluconeogenesis, and force reliance on lipid catabolism in the liver. The lack of changes in PDH activity in the muscle led to additional studies concerning how the $\mathrm{CAC}$ is regulated during torpor in this tissue both at the level of regulatory enzymes within the cycle and also of GDH which is another source of substrate for the CAC.

Mammalian muscle tissue is generally composed of several different fiber types that play complementary roles in terms of their functional and metabolic properties. Type I or slow twitch (sometimes referred to as red muscle) is defined by higher levels of mitochondria, the protein myoglobin (imparting a red hue), and is richly supplied with oxygen from abundant capillary networks that flow through the tissue. Fittingly, this fiber type also has higher levels of lipids since $\beta$-oxidation of fatty acids is an aerobic process. In contrast type II muscle fiber (fast twitch) is more dependent on rapid glycolysis to supply its energy needs and often (as in the case of type IIx) does not contain high levels of mitochondria and myoglobin needed to engage (Cassens \& Cooper, 1971). These muscle fibers are designed for conditions requiring the generation of a large amount of force, but they become more rapidly fatigued. As such these fiber types are very different in their metabolic qualities. Muscles demonstrating a more rapid contraction time were noted to be more white in colour compared to those that were contracted more slowly which appeared to be red and were often called red and white muscles (Scott, Stevens, \& BinderMacCleod, 2001). It has been long known that the degree of composition of muscle fibers can be altered by use. For example, weight-lifters are noted to have a higher proportion of type II fibers 
to generate sufficient strength to lift heavy weights while endurance athletes have greater levels of type I muscle fibers, although the degree to which interconversion can occur between these two types of muscle is contested (Wilson et al., 2012). In the 13-lined ground squirrel, there is an observed shift in muscle composition to type I muscle fibers during torpor through the effects of PGC-1 $\alpha$ signaling to help exploit the abundance of lipid in hibernation (Xu et al., 2013). This was consistent with earlier work which demonstrated a significant and large (3.1-fold) increase in the expression of PGC-1 $\alpha$ during hibernation in the 13-lined ground squirrel during (Eddy, Morin, \& Storey, 2005). While the relative composition of white to red muscle fibers has not been examined in the Richardson's ground squirrel during torpor, the composition of the tissue samples used in these experiments could be determined through immunoblotting to quantify different markers of the muscle fiber differentiation including the abundance of myoglobin, cytochrome $\mathrm{c}$ since these proteins are much more abundant in the mitochondria rich type I (red) muscle. Overall, the skeletal muscles appear to be modified to prefer consumption of lipid substrates over carbohydrates during hibernation even though lipid catabolism itself is reduced in hibernating muscle (Chazarin et al., 2019), in line with the reduction in activity of CAC enzymes observed here.

Mitochondria are critical organelles in generating energy for the cell for they house the ultimate steps in aerobic respiration and are the main source of cellular ATP. Hence, mitochondria and the $\mathrm{CAC}$ are crucial targets for metabolic regulation (Hadj-Moussa, Green, \& Storey, 2018). The reactions carried out in the CAC occur in the mitochondrial matrix, generating the reducing cofactors needed for the establishment of the chemiosmotic gradient across the inner membrane that drives ATP production. In the hepatocyte mitochondria of euthermic, but not hibernating, ground squirrels, a phase change occurs in the lipid bilayer below 
$23{ }^{\circ} \mathrm{C}$ to the gel state as evidenced by the activity of succinate dehydrogenase, a membrane bound enzyme active involved in both the CAC and the ETC (complex II) (Raison \& Lyons, 1971). This observation implies that alterations to the composition of the lipid bilayer in mitochondrial membranes occurs during hibernation that allow the membranes to remain functional even under very low temperatures. This is likely a consequence of the changes in chemical composition of the lipid bilayer in liver mitochondria during hibernation, notably an increase in the levels of cholesterol are important in maintaining the membrane in the liquid state and preventing phase transitions (Perepelkina, Fialkovskaya, \& Kolomiytseva, 2017). This further suggests that even in the liver (where PDH activity was demonstrated to be greatly reduced during hibernation; Chapter 2), the mitochondria need to remain functional. Changes in mitochondrial lipid composition have also been observed in the brains of a hibernating hamster species (Mesocricetus auratus), with a notable increase in monounsaturated fatty acid (particularly $\mathrm{C} 16$ and $\mathrm{C} 18$ ) content during hibernation that would increase the fluidity of the membrane at lower temperatures (Demediuk \& Moscatelli, 1983). Additionally the role of dietary polyunsaturated fatty acids (particularly linoleic acid) in promoting torpor and decreases in body temperature have been well-documented through there action to prevent cardiac arrhythmias via helping to maintain proper cytosolic $\mathrm{Ca}^{2+}$ balance (Ruf \& Arnold, 2008). In general in WAT and muscle tissue hibernators tend to conserve unsaturated fatty acids to retain membrane fluidity at lower temperatures.

Despite the alterations of lipid composition, in order to maintain mitochondrial function in the liver, the overall rate of the mitochondrial activity during hibernation is known to be greatly reduced. This reduction in mitochondrial activity is best associated with the liver mitochondria, where ADP-stimulated state III respiration was significantly reduced during torpor 
relative to summer active controls and was restored during inter-bout arousal when succinate was used as a substrate (Martin, Maniero, Carey, \& Hand, 1999; Staples \& Brown, 2008). Inhibition of liver mitochondrial respiration is thought to occur at the succinate oxidation step during torpor (Fedotcheva, Sharyshev, Mironova, \& Kondrashova, 1985). The results from Chapter 2 of this thesis suggest that, in addition to this internal control over the CAC in the liver, the inhibition of PDH through phosphorylation during torpor is very important and would limit/regulate the entry of carbohydrate (pyruvate) substrate into the CAC. The literature on mitochondrial respiration in hibernation suggests that inhibition of respiration is more pronounced in liver than the muscle, where only modest suppression was observed (Staples, 2014). In liver mitochondria of the 13lined ground squirrel a 70\% reduction in state III respiration was observed in torpor, but only a $30 \%$ reduction occurred in skeletal muscle (Brown, Chung, Belgrave, \& Staples, 2012). This is consistent with the observations in this thesis, which demonstrated a modest reduction in the activity of CS and decreased affinity of KGDC for CoA that could be partly responsible for these changes.

Because of the cyclic nature of torpor and the need for rewarming during every inter-bout arousal, PTMs represent a logical way to control metabolic processes because PTMs can quickly alter protein/enzyme function and can be reversed equally quickly; hence, they represent an energetically efficient mechanism for the fine-tuning of metabolism (Friso \& Van Wijk, 2015). This is in contrast to changing protein/enzyme expression patterns between the euthermic and hibernating states that would be relatively slow in response and also would consume valuable metabolic fuel in the act of turning over protein in repeated synthesis and degradation following the torpor-arousal cycle (Velickovska, Lloyd, Qureshi, \& Van Breukelen, 2005). PTM alterations of ETC complexes have been observed during interbout arousal as compared to torpid animals in 
the liver of 13-lined ground squirrels. Notably, an increase in phosphorylation of the $75 \mathrm{kDa}$ subunit of complex I was observed in the torpid state and was found have a lower activity that could be reversed with in vitro alkaline phosphatase treatment (Mathers \& Staples, 2019). It is perhaps because of the necessity of rapidly reversible regulation during the torpor-arousal cycle, that none of the physical amounts of the enzymes measured in this thesis were observed to change between the euthermic and hibernating animals.

Consequently, it is not very surprising that PTMs are used as a means of mediating metabolic control. However, what is interesting here in this thesis is the identity of some of the PTMs that changed between euthermic control and hibernating animals. Phosphorylation is the best characterized PTM and has been implicated in regulating a wide range of enzymes through either activation or inhibition of activity and is typically found associated with amino acids that have hydroxyl bearing side chains (i.e. Ser, Thr, Tyr) (Humphrey, James, \& Mann, 2015). While changes in phosphorylation state were observed in for KGDH (specifically an increase in tyrosine phosphorylation on all of the subunits was notable) (Green \& Storey, 2020) and PDH in the liver on highly conserved inhibitory residues (Green \& Storey, 2021), a number of other less well-characterized PTMs were also altered during hibernation. For example, both the KGDH E1 subunit and CS showed altered succinylation. This may be related with the location of the enzymes examined here. All of the enzymes studied in this thesis are located within the mitochondria, which has a unique place in cellular metabolism. Specifically, the mitochondria is the location of $\beta$-oxidation, the CAC, and the ETC and contains many metabolites that are either unique or at much higher levels than the rest of the cell (Frezza, 2017). Crucially, acyl-CoA derivatives that often affect proteins through various acylation PTMs, notably succinylation, glutarylation, malonylation and others are found at much higher levels in the mitochondria than 
in the rest of cell (Hong et al., 2016). Interestingly, in the case of KGDH E1 and CS, opposite trends were observed during the hibernating animals with increased succinylation observed on the KGDH E1 subunit and a decrease on CS. This is an interesting observation and was hypothesized to be due to the role of KGDH in generating succinyl-CoA and also that it may function as a succinyl-transferase for both itself and other proteins (Gibson et al., 2015). It could be that during hibernation an increase in self-succinylation decreases the capacity for other proteins to be succinylated by reducing the available succinyl CoA pools. Additionally, the reduction in affinity for CoA observed in KGDC from hibernating animals might reduce activity through this locus in the $\mathrm{CAC}$ and thereby further reduce the amount of succinyl CoA available to succinylate other proteins. Also, the increase in SIRT5 (a prominent mitochondrial deacylase) in the hibernator tissue could contribute to the reduction in CS lysine-succinylation.

Levels of ADP-ribosylation (ADPR) were found to be increased in muscle tissue from hibernators on the E1 subunit (OGDH) of KGDC and also on GDH. ADPR takes two forms in organisms: mono-ADP-ribosylation (MARylation), and poly-ADP-ribosylation (PARylation). PARylation consists of repeated ADP units (sometimes as many as $\sim 200$ ), potentially greatly adding bulk to the protein (Gagné, Shah, \& Poirier, 2001). Differentiating between PARylation and MARylation has been challenging using high-throughput methods because of the heterogeneous size of PARylation and the lability of the bonds between ADP units during fragmentation using mass spectrometry (Vivelo \& Leung, 2015). Although the ADP binding reagent used in this thesis does not distinguish between the two forms of ADPR, it seems unlikely that PARylation is responsible for the changes observed on western blots because the proteins explored here were at the expected molecular weights for OGDH and GDH and western blots showed clear well-defined bands. For comparison, it is thought that the average chain 
length of PARylation is around 10 units $(\sim 5.5 \mathrm{kDa})$ and, if present, should create obvious decrease in electrophoretic mobility of the protein (Vivelo \& Leung, 2015). Additionally, MARylation is thought to have a greater diversity of sites than PARylation, with some estimates suggesting as many as 700 times more MARylated sites than PARylated sites in the cell. This makes uncharacterized MARylated sites much more likely to occur on these two proteins and emphasizes the importance of this understudied PTM (Jacobson et al., 1984; Wielckens, Bredehorst, Adamietz, \& Hilz, 1981). Therefore, it seems likely that MARylation is responsible for the signal observed on GDH and OGDH bands that increased in hibernating animals. Since both of these proteins are mitochondrial proteins, it seems likely that a common mechanism may exist for exerting control over this PTM. The increase in GDH and OGDH ADPR could be the result of a few different explanations. One possibility could be a change in the ratio between $\mathrm{NAD}^{+}$and NADH since ADP-ribosyl transferases (ARTs) require $\mathrm{NAD}^{+}$as a substrate for transfer of an ADP-ribose moiety. The increase in ADPR could potentially be explained by an increase in the ratio of $\mathrm{NAD}^{+} / \mathrm{NADH}$ during hibernation. Typically the ratio of $\mathrm{NAD}^{+} / \mathrm{NADH}$ in the mitochondrion remains relatively low, between 7 to 8 , compared to the cytosolic fraction where it ranges widely between 60 to 700 (Veech, Guynn, \& Veloso, 1972; Williamson, Lund, \& Krebs, 1967). To date, the redox balance state of the mitochondria in hibernating animals is unknown, but it has been suggested that the metabolic changes accompanying hibernation could result in an increased ratio of $\mathrm{NAD}^{+} / \mathrm{NADH}$ (Klug \& Brigham, 2015; Staples \& Brown, 2008). This would be consistent with a reduction in glycolysis limiting the amount of NADH produced in the mitochondria and could thereby affect a positive feedback loop by altering the structure and functional properties of CAC enzymes such as OGDH and further reducing the production of NADH. Additionally, increased $\mathrm{NAD}^{+}$levels would also, in theory, increase the deacylase 
activity of mitochondrial SIRT5, as seen in the case of CS observed here with its lessened levels of succinylation (Chapter 3). Indeed, this could be a common response in hypometabolic states as evidenced by another study that looked at the mitochondrial enzyme carbamoyl phosphate synthetase I in the freeze-tolerant wood frog that demonstrated a decrease in the levels of lysineglutarylation (another PTM removed through SIRT5 activity) in the liver in response to wholebody freezing (Green \& Storey, 2018). This state is similar to hibernation in that both are characterized by pronounced reduction in cellular respiration and in particular glycolysis (Smolinski, Mattice, \& Storey, 2017; Storey, 1987). The increased sensitivity to NADH inhibition by CS demonstrated in Chapter 3 would be an interesting adaptation to the hibernating condition if the $\mathrm{NAD}^{+} / \mathrm{NADH}$ ratio is indeed increased since this could potentially offset some of the changes in the redox balance within the mitochondrial matrix and ensure inhibition of CS even when NADH is depleted.

This thesis has demonstrated that research into the roles of PTMs in modulating the activity of central metabolic pathways is still ongoing. While the identification of protein phosphate in the early $20^{\text {th }}$ century (Levene \& Alsberg, 1906), the diverse array of PTMs (over 200 biologically relevant PTMs) makes it difficult to assess the functional significance of these crucial protein modifications (Duan \& Walther, 2015). This thesis aimed to characterize the role of PTMs in mediating enzymatic regulation, however it was limited in its scope due to the bewildering diversity of PTMs that are found in nature and the tools available to assess them. For this reason, commonly observed PTMs were preferentially assessed. Phosphorylation, widely regarded as the most common PTM, was the first assessed due to its ubiquity (Vlastaridis et al., 2017). The availability of protocols to activate endogenous protein kinases, also makes this an ideal PTM to investigate, as this allows for confirmation of the functional role of these PTMs on 
enzyme activity. From there, the workflow used here emphasized assessing other common PTMs (such as lysine acetylation and methylation) and those that are known to be present from other high-throughput studies on each specific enzyme. This allows for a more targeted approach rather than simply randomly choosing PTMs in the hope that some of them might be altered in response to hibernation. The downside to this approach is that it could potentially miss rarer PTMs, or ones that are less well characterized, but might have equally important functional properties. For example, the extreme diversity of glycosylation events (particularly with Olinked glycosylation) means that it is difficult to assess the degree and impact of glycosylation using the immunoblotting techniques described in this thesis (Schoberer et al., 2018). In principle, glycosylation of mitochondrial proteins is a relatively rare event as this process typically occurs within the endoplasmic reticulum and therefore are often critical in secretory proteins (Smeekens, Xiao, \& Wu, 2017). However, the functional significance of glycosylation is deeply important to many cell functions and alterations to mitochondrial glycosylation patterns have even been linked to the development of Alzheimer's disease in rats (Yu et al., 2017). The subcellular location also plays an important role in the choice of which PTMs were investigated owing to the fact that some PTMs are more common in certain areas of the cell, with the mitochondrial enzymes examined here tending towards having a higher degree of lysine acylations (acetylation, succinylation, glutarylation etc.). Therefore, while efforts were made to assess the relative degree of PTMs that were most likely to be present on the proteins, the results here should be tempered with the understanding that numerous other PTMs were not assessed that could also have important regulatory roles in the function of the enzymes examined. 


\subsection{Conclusion}

The work performed in this thesis demonstrated strong evidence for metabolic regulation at the level of the CAC in muscle of hibernating Richardson's ground squirrels. Interestingly no changes were noted in PDH activity or phosphorylation status in the muscle (although a substantial reduction occurred in liver), suggesting that regulation of the entry of carbohydrate substrates into the CAC does not occur at this stage but rather is likely under the control of glycolysis. Regulation of the CAC appears to be controlled by changes in the kinetic parameters of two of the irreversible steps within the CAC; CS and KGDC. In the case of CS a stable decrease in the activity of the enzyme was noted at the three temperatures tested and for KGDC a reduction in enzyme affinity for CoA substrate was demonstrated. Both of these changes suggest decreased catabolic function of the CAC during hibernation. GDH was shown to have a greatly decreased affinity for its substrate, glutamate, at $8{ }^{\circ} \mathrm{C}$ for the euthermic, but not hibernating, form of the enzyme. This suggests an adaptation to maintain activity during hibernation, potentially as a means to tap into the increased circulating glutamate levels during arousal as a fuel source. Interestingly, none of the enzymes showed changes in their protein levels in the tissues during hibernation. This was as predicted by the hypothesis that altered PTMs on enzymes would be responsible for regulating metabolism of the animal during hibernation rather than energetically costly fluctuations in protein levels. Accordingly, immunoblotting of purified enzymes revealed notable changes in the PTM status of all proteins studied. Notably, an increase in ADPR on the OGDH subunit of KGDC and GDH, as well as increases in succinylation on OGDH, and decreases in this same PTM on CS were observed. Taken together, these results demonstrated that regulation of central metabolism requires balancing the opposing demands of maintaining the crucial processes essential for the catabolism of fatty acids as fuel during hibernation while 
also effecting generalized metabolic rate reduction necessary to prevent the wasting of finite fuel supplies.

\subsection{Future Directions}

The changes occurring in mitochondrial metabolism during torpor lead to additional largerscale questions concerning the potential impact on the PTM landscape in this central organelle. The studies here demonstrated several trends in PTM alterations of the enzymes mediating the CAC and peripheral pathways. The notable increase in lysine succinylation of OGDH on the enzyme producing succinyl $\mathrm{CoA}(\mathrm{OGDH})$ and the decrease in the same PTM on CS suggest that perturbations of this PTM occur in the mitochondria during hibernation. This was supported by the significant increase in the expression of SIRT5, the enzyme catalyzing deacylation of various acyl groups including succinylation in the mitochondria. Additionally, the increase in ADPR on both GDH and OGDH during hibernation suggests that the mechanisms governing the overall levels of this PTM are modified during hibernation. These observations could lead to additional studies focusing on identifying other proteins that are similarly modified in the mitochondria of skeletal muscle during hibernation. These changes could be investigated using immunoprecipitation of proteins with antibodies recognizing these PTMs and then identification of the proteins could be undertaken using 2D gel electrophoresis and subsequent mass spectroscopy. These experiments could give valuable insights into understanding the targets of these PTMs that are not as well characterized as some others, such as phosphorylation. Such studies could then be expanded to examine other acylations such as acetylation, malonylation, and glutarylation which are beginning to be understood to have important roles in mitochondrial physiology (Ringel, Tucker, \& Haigis, 2018). 
The role of $\mathrm{CAC}$ cycle regulation during metabolic rate depression has not been as thoroughly explored as other metabolic pathways in animal models of hypometabolism. One particular metabolic stress that would be of interest is anoxia/hypoxia since the CAC in its complete form is an aerobic process and intersects with the ETC at succinate dehydrogenase (Complex II in the ETC). Under hypoxic conditions, the function of the CAC would likely be substantially altered and is commonly assumed to take an altered form that results in the accumulation of succinate in vivo through two sections of the cycle running in opposite directions and converging on succinate (Chinopoulos, 2013). While few mammals can survive true anoxia for extended periods of time, several species of frog and turtle species are anoxia tolerant as they overwinter underwater and survive for months without oxygen (Al-Attar \& Storey, 2018; Krivoruchko \& Storey, 2010). Indeed, in one turtle species anoxia is associated with accumulation of succinate suggesting altered CAC functionality (Bundgaard et al., 2019). Investigating the regulation of CAC enzymes in animals capable of robust anoxia tolerance would help to give a better picture of how animal mitochondria cope with this hypometabolic, oxygen-deprived state. 


\section{Chapter 6 References}

Abnous, K., \& Storey, K. B. (2008). Skeletal muscle hexokinase: Regulation in mammalian hibernation. Molecular and Cellular Biochemistry, 319(1-2), 41-50. https://doi.org/10.1007/s11010-008-9875-5

Al-Attar, R., \& Storey, K. B. (2018). Effects of anoxic exposure on the nuclear factor of activated T cell (NFAT) transcription factors in the stress-tolerant wood frog. Cell Biochemistry and Function, 36(8), 420-430. https://doi.org/10.1002/cbf.3362

Berg, J. M., Tymoczko, J. L., \& Stryer, L. (2002). Triacylglycerols Are Highly Concentrated Energy Stores. Biochemistry. Retrieved from papers2://publication/uuid/7EB6183C-F1A34903-A72F-B1A659CECF68

Brooks, S. P. J., \& Storey, K. B. (1992). Mechanisms of glycolytic control during hibernation in the ground squirrel Spermophilus lateralis. Journal of Comparative Physiology B, 162(1), 23-28. https://doi.org/10.1007/BF00257932

Brown, J. C. L., Chung, D. J., Belgrave, K. R., \& Staples, J. F. (2012). Mitochondrial metabolic suppression and reactive oxygen species production in liver and skeletal muscle of hibernating thirteen-lined ground squirrels. American Journal of Physiology - Regulatory Integrative and Comparative Physiology, 302(1), R15-28. https://doi.org/10.1152/ajpregu.00230.2011

Buck, C. L., \& Barnes, B. M. (2000). Effects of ambient temperature on metabolic rate, respiratory quotient, and torpor in an arctic hibernator. American Journal of Physiology Regulatory Integrative and Comparative Physiology, 279, R255-R262. https://doi.org/10.1152/ajpregu.2000.279.1.r255

Bundgaard, A., James, A. M., Gruszczyk, A. V., Martin, J., Murphy, M. P., \& Fago, A. (2019). Metabolic adaptations during extreme anoxia in the turtle heart and their implications for ischemia-reperfusion injury. Scientific Reports, 9(1). https://doi.org/10.1038/s41598-01939836-5

Cassens, R. G., \& Cooper, C. C. (1971). Red and white muscle. Advances in Food Research, 19, 1-74. https://doi.org/10.1016/S0065-2628(08)60030-0

Chazarin, B., Storey, K. B., Ziemianin, A., Chanon, S., Plumel, M., Chery, I., ... Bertile, F. (2019). Metabolic reprogramming involving glycolysis in the hibernating brown bear skeletal muscle. Frontiers in Zoology, 16(1), 12. https://doi.org/10.1186/s12983-019-0312-2

Chinopoulos, C. (2013). Which way does the citric acid cycle turn during hypoxia? The critical role of $\alpha$-ketoglutarate dehydrogenase complex. Journal of Neuroscience Research, 91(8), 1030-1043. https://doi.org/10.1002/jnr.23196

Dark, J. (2005). Annual lipid cycles in hibernators: Integration of physiology and behavior. Annual Review of Nutrition, 25(1), 469-497. https://doi.org/10.1146/annurev.nutr.25.050304.092514 
Demediuk, P., \& Moscatelli, E. A. (1983). Synaptosomal and brain mitochondrial lipids in hibernating and cold-acclimated golden hamsters. Journal of Neurochemistry, 40(4), 11001105. https://doi.org/10.1111/j.1471-4159.1983.tb08098.x

Duan, G., \& Walther, D. (2015). The roles of post-translational modifications in the context of protein interaction networks. PLoS Computational Biology, 11(2). https://doi.org/10.1371/journal.pcbi.1004049

Eddy, S. F., Morin, P., \& Storey, K. B. (2005). Cloning and expression of PPAR-gamma and PGC-1alpha from the hibernating ground squirrel, Spermophilus tridecemlineatus. Molecular \& Cellular Biochemistry, 269(1-2), https://doi.org/175-182. doi: 10.1007/s11010-005-3459-4

Fedotcheva, N. J., Sharyshev, A. A., Mironova, G. D., \& Kondrashova, M. N. (1985). Inhibition of succinate oxidation and $\mathrm{K}+$ transport in mitochondria during hibernation. Comparative Biochemistry and Physiology -- Part B: Biochemistry and, 82(1), 191-195. https://doi.org/10.1016/0305-0491(85)90151-8

Florant, G. L. (1998). Lipid metabolism in hibernators: the importance of essential fatty acids. American Zoologist, 38(2), 331-340. https://doi.org/10.1093/icb/38.2.331

Frezza, C. (2017). Mitochondrial metabolites: Undercover signalling molecules. Interface Focus. https://doi.org/10.1098/rsfs.2016.0100

Friso, G., \& Van Wijk, K. J. (2015). Posttranslational protein modifications in plant metabolism. Plant Physiology, 169(3), 1469-1487. https://doi.org/10.1104/pp.15.01378

Gagné, J. P., Shah, R. G., \& Poirier, G. G. (2001). Analysis of ADP-ribose polymer sizes in intact cells. Molecular and Cellular Biochemistry, 224(1-2), 183-185. https://doi.org/10.1023/A:1011910329010

Galster, \& Morrison, P. (1970). Cyclic changes in carbohydrate concentrations during hibernation in the arctic ground squirrel. The American Journal of Physiology, 218(4), 1228-1232. https://doi.org/10.1152/ajplegacy.1970.218.4.1228

Galster, W., \& Morrison, P. R. (1975). Gluconeogenesis in arctic ground squirrels between periods of hibernation. American Journal of Physiology, 228(1), 325-330. https://doi.org/10.1152/ajplegacy.1975.228.1.325

Gibson, G. E., Xu, H., Chen, H. L., Chen, W., Denton, T. T., \& Zhang, S. (2015). Alphaketoglutarate dehydrogenase complex-dependent succinylation of proteins in neurons and neuronal cell lines. Journal of Neurochemistry, 134(1), 86-96. https://doi.org/10.1111/jnc.13096

Green, S. R., \& Storey, K. B. (2018). Purification of carbamoyl phosphate synthetase 1 (CPS1) from wood frog (Rana sylvatica) liver and its regulation in response to ice-nucleation and subsequent whole-body freezing. Molecular and Cellular Biochemistry, 455, $29-39$. https://doi.org/10.1007/s11010-018-3468-8 
Green, S. R., \& Storey, K. B. (2020). Regulation of the $\alpha$-ketoglutarate dehydrogenase complex during hibernation in a small mammal, the Richardson's ground squirrel (Urocitellus richardsonii). Biochimica et Biophysica Acta - Proteins and Proteomics, 1868(9). https://doi.org/10.1016/j.bbapap.2020.140448

Green, S. R., \& Storey, K. B. (2021). Functional and post-translational characterization of pyruvate dehydrogenase demonstrates repression of activity in the liver but not skeletal muscle of the Richardson's ground squirrel (Urocitellus richardsonii) during hibernation. Journal of Thermal Biology. https://doi.org/10.1016/j.jtherbio.2021.102996

Hadj-Moussa, H., Green, S. R., \& Storey, K. B. (2018). The living dead: mitochondria and metabolic arrest. IUBMB Life, 70(2), 1260-1266. https://doi.org/10.1002/iub.1910

Hong, S. Y., Ng, L. T., Ng, L. F., Inoue, T., Tolwinski, N. S., Hagen, T., \& Gruber, J. (2016). The role of mitochondrial non-enzymatic protein acylation in ageing. PLoS ONE, 11(12). https://doi.org/10.1371/journal.pone.0168752

Humphrey, S. J., James, D. E., \& Mann, M. (2015). Protein phosphorylation: A major switch mechanism for metabolic regulation. Trends in Endocrinology and Metabolism. https://doi.org/10.1016/j.tem.2015.09.013

Jacobson, M. K., Michael Payne, D., Alvarez-Gonzalez, R., Juarez-Salinas, H., Sims, J. L., \& Jacobson, E. L. (1984). Determination of in vivo levels of polymeric and monomeric ADPribose by fluorescence methods. Methods in Enzymology, 106(C), 483-494. https://doi.org/10.1016/0076-6879(84)06052-3

Klug, B. J., \& Brigham, R. M. (2015). Changes to metabolism and cell physiology that enable mammalian hibernation. Springer Science Reviews, 3(1), 39-56. https://doi.org/10.1007/s40362-015-0030-x

Krivoruchko, A., \& Storey, K. B. (2010). Forever young: Mechanisms of natural anoxia tolerance and potential links to longevity. Oxidative Medicine and Cellular Longevity. https://doi.org/10.4161/oxim.3.3.12356

Lanaspa, M. A., Epperson, L. E., Li, N., Cicerchi, C., Garcia, G. E., Roncal-Jimenez, C. A., ... Johnson, R. J. (2015). Opposing activity changes in AMP deaminase and AMP-activated protein kinase in the hibernating ground squirrel. PLoS ONE, 10(4). https://doi.org/10.1371/journal.pone.0123509

Levene, P. A., \& Alsberg, C. L. (1906). The cleavage products of vitellin. Journal of Biological Chemistry, 2(1), 127-133. https://doi.org/doi:10.1016/S0021-9258(17)46054-6

Martin, S. L., Maniero, G. D., Carey, C., \& Hand, S. C. (1999). Reversible depression of oxygen consumption in isolated liver mitochondria during hibernation. Physiological and Biochemical Zoology, 72(3), 255-264. https://doi.org/10.1086/316667

Mathers, K. E., \& Staples, J. F. (2019). Differential posttranslational modification of mitochondrial enzymes corresponds with metabolic suppression during hibernation. 
American Journal of Physiology-Regulatory, Integrative and Comparative Physiology, 317(2), R262-R269. https://doi.org/10.1152/ajpregu.00052.2019

Mrosovsky, N. (1974). Hypothalamic hyperphagia without plateau in ground squirrels. Physiology and Behavior, 12(2), 259-264. https://doi.org/10.1016/0031-9384(74)90180-2

Perepelkina, N. I., Fialkovskaya, L. A., \& Kolomiytseva, I. K. (2017). The effect of hibernation on lipids of the liver mitochondrial fraction in the Yakut ground squirrel Spermophilus undulatus. Biophysics (Russian Federation), 62(2), 233-239. https://doi.org/10.1134/S0006350917020178

Raison, J. K., \& Lyons, J. M. (1971). Hibernation: alteration of mitochondrial membranes as a requisite for metabolism at low temperature. Proceedings of the National Academy of Sciences of the United States of America, 68(9), 2092-2094. https://doi.org/10.1073/pnas.68.9.2092

Ringel, A. E., Tucker, S. A., \& Haigis, M. C. (2018). Chemical and physiological features of mitochondrial acylation. Molecular Cell, 72(4), 610-624. https://doi.org/10.1016/j.molcel.2018.10.023

Ruf, T., \& Arnold, W. (2008) Effects of polyunsaturated fatty acids on hibernation and torpor: a review and hypothesis. American Journal of Physiology Regulatory, Integrative and Comparative Physiology, 294, R1044-R1052. https://doi.org/doi:10.1152/ajpregu.00688.2007

Scott, W., Stevens, J., \& Binder-Macleod, S. A. (2001) Human skeletal muscle fiber type classifications. Physical Therapy \& Rehabilitation Journal, 81(11), 1810-1816. https://doi.org/10.1016/S0065-2628(08)60030-0

Schoberer, J., Shin, Y., Vavra, U., Veit, C., \& Strasser, R. (2018). Analysis of protein glycosylation in the ER. Methods in Molecular Biology, 1691, 205-222. https://doi.org/10.1007/978-1-4939-7389-7_16

Shimozuru, M., Kamine, A., \& Tsubota, T. (2012). Changes in expression of hepatic genes involved in energy metabolism during hibernation in captive, adult, female Japanese black bears (Ursus thibetanus japonicus). Comparative Biochemistry and Physiology - B Biochemistry and Molecular Biology, 163(2), 254-261. https://doi.org/10.1016/j.cbpb.2012.06.007

Smeekens, J., Xiao, H., \& Wu, R. (2017). Global analysis of secreted proteins and glycoproteins in Saccharomyces cerevisiae. Journal of Proteome Research, 16(2), 1039-1049. https://doi.org/10.1021/acs.jproteome.6b00953

Smolinski, M. B., Green, S. R., \& Storey, K. B. (2020). Characterizing the regulation of pyruvate kinase in response to hibernation in ground squirrel liver (Urocitellus richardsonii). Comparative Biochemistry and Physiology Part - B: Biochemistry and Molecular Biology, 248-249. https://doi.org/10.1016/j.cbpb.2020.110466 
Smolinski, M. B., Mattice, J. J. L., \& Storey, K. B. (2017). Regulation of pyruvate kinase in skeletal muscle of the freeze tolerant wood frog, Rana sylvatica. Cryobiology, 77, 25-33. https://doi.org/10.1016/j.cryobiol.2017.06.002

Staples, J. F. (2014). Metabolic suppression in mammalian hibernation: The role of mitochondria. Journal of Experimental Biology, 217(12), 2032-2036. https://doi.org/10.1242/jeb.092973

Staples, J. F., \& Brown, J. C. L. (2008). Mitochondrial metabolism in hibernation and daily torpor: A review. Journal of Comparative Physiology B: Biochemical, Systemic, and Environmental Physiology, 178(7), 811-827. https://doi.org/10.1007/s00360-008-0282-8

Staples, J. F., \& Hochachka, P. W. (1998). The effect of hibernation status and cold-acclimation on hepatocyte gluconeogenesis in the golden-mantled ground squirrel (Spermophilus lateralis). Canadian Journal of Zoology, 76(9), 1734-1740. https://doi.org/10.1139/cjz-769-1734

Storey, K. B. (1987). Glycolysis and the regulation of cryoprotectant synthesis in liver of the freeze tolerant wood frog. Journal of Comparative Physiology B, 157(3), 373-380. https://doi.org/10.1007/BF00693364

Tashima, L. S., Adelstein, S. J., \& Lyman, C. P. (1970). Radioglucose utilization by active, hibernating, and arousing ground squirrels. The American Journal of Physiology, 218(1), 303-309. https://doi.org/10.1152/ajplegacy.1970.218.1.303

Veech, R. L., Guynn, R., \& Veloso, D. (1972). The time-course of the effects of ethanol on the redox and phosphorylation states of rat liver. The Biochemical Journal, 127(2), 387-397. https://doi.org/10.1042/bj1270387

Velickovska, V., Lloyd, B. P., Qureshi, S., \& Van Breukelen, F. (2005). Proteolysis is depressed during torpor in hibernators at the level of the 20S core protease. Journal of Comparative Physiology B: Biochemical, Systemic, and Environmental Physiology, 175(5), 329-335. https://doi.org/10.1007/s00360-005-0489-X

Vivelo, C. A., \& Leung, A. K. L. (2015). Proteomics approaches to identify mono-(ADPribosyl)ated and poly(ADP-ribosyl)ated proteins. Proteomics, 15(2-3), 203-217. https://doi.org/10.1002/pmic.201400217

Vlastaridis, P., Kyriakidou, P., Chaliotis, A., Van de Peer, Y., Oliver, S. G., \& Amoutzias, G. D. (2017). Estimating the total number of phosphoproteins and phosphorylation sites in eukaryotic proteomes. Gigascience, 6(2), 1-11. https://doi.org/10.1093/gigascience/giw015

Wang, L. C. H. (1979). Time patterns and metabolic rates of natural torpor in the Richardson's ground squirrel. Canadian Journal of Zoology, 57(2), 149-155. https://doi.org/10.1139/z79012

Wielckens, K., Bredehorst, R., Adamietz, P., \& Hilz, H. (1981). Protein-bound polymeric and monomeric ADP-ribose residues in hepatic tissues: Comparative analyses using a new 
procedure for the quantification of poly(ADP-ribose). European Journal of Biochemistry, 117(1), 69-74. https://doi.org/10.1111/j.1432-1033.1981.tb06303.x

Williamson, D. H., Lund, P., \& Krebs, H. A. (1967). The redox state of free nicotinamideadenine dinucleotide in the cytoplasm and mitochondria of rat liver. The Biochemical Journal, 103(2), 514-527. https://doi.org/10.1042/bj1030514

Wilson, J. M., Loenneke, J. P., Jo, E., Wilson, G. J., Zourdos, M. C., \& Kim J. S. (2012). The effects of endurance, strengthm and power training on muscle fiber type shifting. Journal of Strength and Conditioning Research, 26(6), 1724-1729.

https://doi.org/10.1519/JSC.0b013e318234eb6f

Xu, R., Andres-Mateos, E., Mejias, R., MacDonald, E. M., Leiwand L. A., Merriman, D. K., Fink, R. H. A., \& Cohn, R. D. (2013). Hibernating squirrel muscle activates the endurance exercise pathway despite prolonged immobilization. Experimental Neurology, 247, 392401. https://doi.org/ 10.1016/j.expneurol.2013.01.005

Yu, H., Yang, C., Chen, S., Huang, C., Liu, C., Liu, J., \& Yin, W. (2017). Comparison of the glycopattern alterations of mitochondrial proteins in cerebral cortex between rat Alzheimer's disease and the cerebral ischemia model. Scientific Reports, 7, 39948.

https://doi.org/10.1038/srep39948 


\section{Appendices}


Appendix A: Chapter 3 Supplementary Data and Figures 
Appendix A.1. Standard curve of coenzyme A amounts plotted against absorbance at $412 \mathrm{~nm}$ with $0.1 \mathrm{mM}$ DTNB in a final volume of $200 \mu \mathrm{L} 50 \mathrm{mM}$ Tris buffer $\mathrm{pH} 8.0$ at $22^{\circ} \mathrm{C}$. The slope here was used to convert Abs/s in the assays to $\mathrm{mU}$ of CS activity defined as the amount of activity that converted $1 \mu \mathrm{Mol}$ of substrate to product per minute. Each point represents the average absorbance in two identically prepared wells.

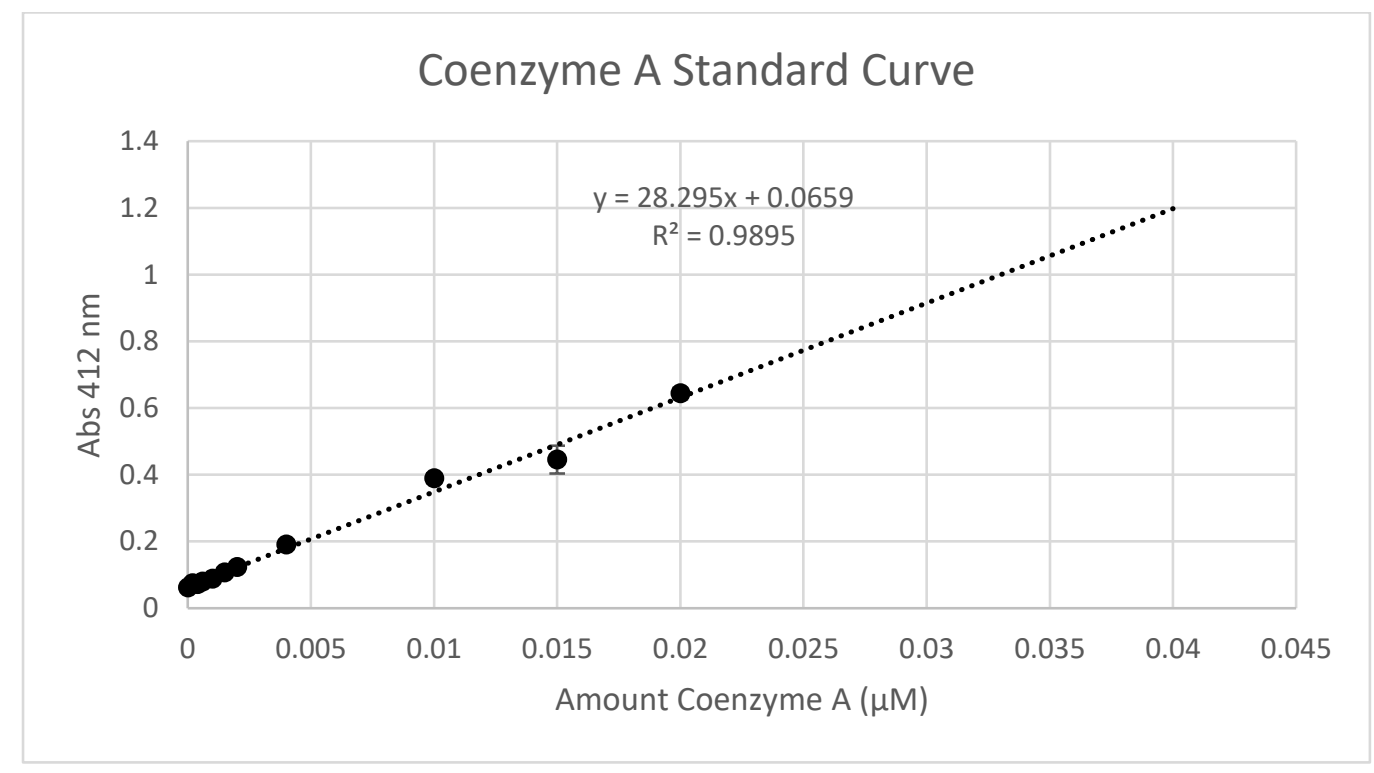


Appendix A.2. Sequences and alignment between 13-lined ground squirrel CS (Ictidomys tridecemlineatus) and human (Homo sapiens). Lysine residues known to be succinylated in the human sequence are highlighted in yellow and underlined, the known regulatory lysine methylation

>XP_005335687.1 citrate synthase, mitochondrial [Ictidomys tridecemlineatus]

MALLTAAARLLGAKNSSCLVLAARHASTSTTNLKDILANLIPKEQARIKAFRQQHGKTVVGQITVDMMYG GMRGMKGLVYETSVLDPDEGIRFRGHSIPECQKLLPKAKGGEEPLPEGLFWLLVTGQIPTEEQVSWLSKE WAKRAALPSHVVTMLDNFPTNLHPMSQLSAAITALNSESNFARAYAEGINRTKYWELIYEDCMDLIAKLP CVAAKIYRNLYREGSSIGAIDSKLDWSHNFTNMLGYTDSQFTELMRLYLTIHSDHEGGNVSAHTSHLVGS ALSDPYLSFAAAMNGLAGPLHGLANQEVLVWLTQLQKEVGKDVSDEKLRDYIWNTLNSGRVVPGYGHAVL RKTDPRYSCQREFALKHLPNDPMFKLVAQLYKIVPNILLEQGKAKNPWPNVDAHSGVLLQYYGMTEMNYY TVLFGVSRALGVLAQLIWSRALGFPLERPKSMSTDGLMKFVDSKSG

>AAQ13428.1 citrate synthase [Homo sapiens]

MALLTAAARLLGTKNASCLVLAARHASASSTNLKDILADLIPKEQARIKTFRQQHGKTVVGQITVDMMYG GMRGMKGLVYETSVLDPDEGIRFRGFSIPECQKLLPKAKGGEEPLPEGLFWLLVTGHIPTEEQVSWLSKE WAKRAALPSHVVTMLDNFPTNLHPMSQLSAAVTALNSESNFARAYAQGISRTKYWELIYEDSMDLIAKLP CVAAKIYRNLYREGSGIGAIDSNLDWSHNFTNMLGYTDHQFTELTRLYLTIHSDHEGGNVSAHTSHLVGS AFSDPYLSFAAAMNGLAGPLHGLANQEVLVWLTQLQKEVGKDVSDEKLRDYIWNTLNSGRVVPGYGHAVL RKTDPRYTCQREFALKHLPNDPMFKLVAQLYKIVPNVLLEQGKAKNPWPNVDAHSGVLLQYYGMTEMNYY TVLFGVSRALGVLAQLIWSRALGFPLERPKSMSTEGLMKFVDSKSG 
Appendix A.3. Full blot image demonstrating the molecular weight of citrate synthase from euthermic and hibernating $U$.richardsonii muscle tissue lysates. The blue arrows indicates the location on the western blot image (on the left slightly above the $42 \mathrm{kDa}$ band). This band was used to quantify the relative total amount of CS in euthermic and hibernating muscle.

CS Western Blot from Euthermic and Hibernating Whole Tissue Lysates

ECL

Coomassie
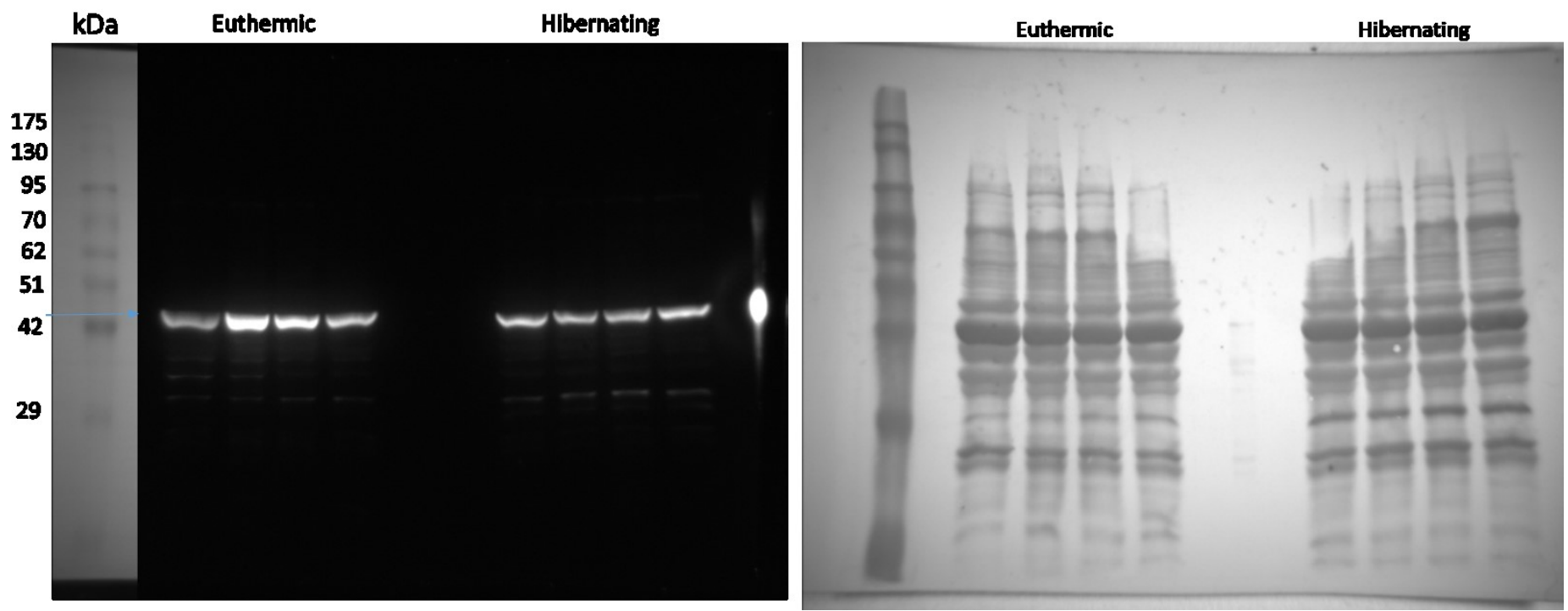


\section{Appendix B: Chapter 4 Supplementary Data and Figures}




\section{Supplementary Bioinformatics Figures}

Supplementary Fig. B.1. Sequence alignment showing OGDH from the 13-lined ground squirrel, Ictidomys tridecemlineatus, to the homologous rat, Rattus norvegicus, sequence. Highlighted glutamate and aspartate residues (blue) were determined to be putative ADP-ribosyl sites based on ADPredict software analysis of the rat sequence; 14 of the 16 total residues were conserved in the 13-lined ground squirrel sequence. Highlighted lysine residues (yellow) have high-throughput experimental evidence as site for succinylation and all were conserved in the ground squirrel sequence. Highlighted tyrosine residues (red) have one or more publications reporting high-throughput evidence for protein phosphorylation and all were also conserved in ground squirrels.

Supplementary Fig. B.2. Sequence alignment showing the DLST sequence of $I$. tridecemlineatus compared to the sequence of $R$. norvegicus. The highlighted serine residue (red) is conserved between the two sequences and has been shown in 10 previous high-throughput studies to be phosphorylated. Below are the potential kinases associated with this position and their scores determined using NetPhos 3.1 prediction software. Three highlighted tyrosine residues (yellow) in the ground squirrel sequence are predicted to be phosphorylated by NetPhos 3.1 software but have no direct supporting experimental evidence. Two of these residues were also conserved in the ground squirrel sequence.

Supplementary Fig. B.3. Sequence alignment showing the DLD sequence of I. tridecemlineatus compared to the sequence of Homo sapiens. The highlighted tyrosine residue is conserved between the two sequences and has been shown in 49 previous high-throughput studies to be phosphorylated in humans. Below are the potential kinases associated with this position (Y153) and their scores determined using NetPhos 3.1 prediction software. 


\section{Supplementary Fig. B.1. (See caption for description on page 197)}

ictidomystridecemlineatus rattusnorvegicus

ictidomystridecemlineatus rattusnorvegicus

ictidomystridecemlineatus rattusnorvegicus

ictidomystridecemlineatus rattusnorvegicus

ictidomystridecemlineatus rattusnorvegicus

ictidomystridecemlineatus rattusnorvegicus

ictidomystridecemlineatus rattusnorvegicus

ictidomystridecemlineatus rattusnorvegicus

ictidomystridecemlineatus rattusnorvegicus

ictidomystridecemlineatus rattusnorvegicus

ictidomystridecemlineatus rattusnorvegicus

ictidomystridecemlineatus rattusnorvegicus

ictidomystridecemlineatus rattusnorvegicus

ictidomystridecemlineatus rattusnorvegicus

ictidomystridecemlineatus rattusnorvegicus

ictidomystridecemlineatus rattusnorvegicus

ictidomystridecemlineatus rattusnorvegicus

ictidomystridecemlineatus rattusnorvegicus
MFHLRTCAAKLRPLTASOTVKTFSONKSAAIRTFOOIRCYSAPVAAEPFLSGTSSNYVEE 60 MFHLRTCAAKLRPLTASQTVKTFSQNKPAAIRTFQQIRCYSAPVAAEPFLSGTSSNYVEE 60

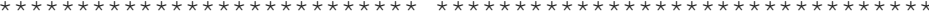

MYCAWLENPKSVHKSWDIFFRNTNAGAPPGTAYQSPLSLSRGSLATVAHAQSLVDAQPNV 120 MYCAWLENPKSVHKSWDIFFRNTNAGAPPGTAYQSPLSLSRSSLATMAHAQSLVEAQPNV 120

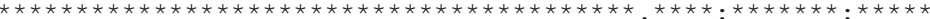

DKLVEDHLAVQSLIRAYQIRGHHVAQLDPLGILDADLDSSVPADI ISSTDKLGFYGLDES 180 DKLVEDHLAVQSLIRAYQIRGHHVAQLDPLGILDADLDSSVPADI ISSTDKLGFYGLHES 180

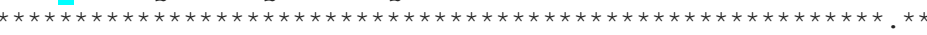

DLDKVFHLPTTTFIGGQESALPLREIIRRLEMAYCQH IGVEFMF INDLEQCQWIRQKFET 240 DLDKVFHLPTTTFIGGQEPALPLREIIRRLEMAYCQHIGVEFMF INDLEQCQWIRQKFET 240

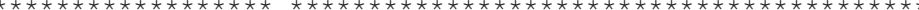

PGIMQFTNEEKRTLLARLVRSTRFEEFLHRKWSSEKRFGLEGCEVLIPALKTIIDKSSEN 300 PGIMOFTNEEKRTLLARLVRSTRFEEFLORKWSSEKRFGLEGCEVLIPALKTI IDMSSAN 300

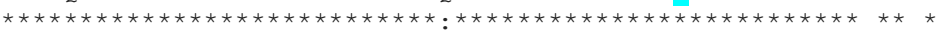

GVDYVIMGMPHRGRLNVLANVIRKELEQIFCQFDSKLEAADEGSGDMKYHLGMYHRRINR 360 GVDYVIMGMP HRGRLNVLANVIRKELEQIFCQFDSKLEAADEGSGDMKYHLGMYHRRINR 360

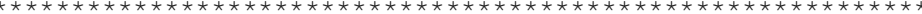

VTDRNITLSLVANPSHLEAADPVVMGKTKAEQFYCGDTEGKKVMSILLHGDAAFAGQGIV 420 VTDRNITLSLVANPSHLEAADPVVMGKTKAEQFYCGDTEGKKVMS ILLHGDAAFAGQGIV 420

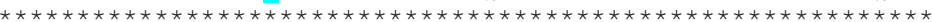

YETFHLSDLPSYTTHGTVHVVVNNQIGFTTDPRMARSSPYPTDVARVVNAPIFHVNADDP 480 YETFHLSDLPSYTTHGTVHVVVNNQIGFTTDPRMARSSPYPTDVARVVNAPIFHVNSDDP 480

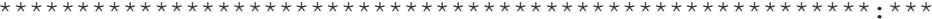

EAVMYVCKVAAEWRSTFHKDVVVDLVCYRRNGHNEMDEPMFTQPLMYKQIRKQKPVLQKY 540 EAVM YVKVAAEWRNTFHKDVVVDLVCYRRNGHNEMDEPMFTQPLM KQIRKQKPVLQKY 540

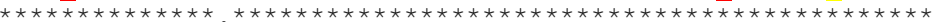

AELLVSQGVVNQPEYEEEISKYDKICEEAFTRSKDEKILHIKHWLDSPWPGFFTLDGQPR 600 AELLVSQGVVNQPEYEEEISKYDKICEEAFTRSKDEKILHIKHWLDSPWPGFFTLDGQPR 600

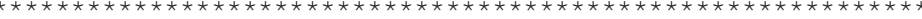

SMSCPSTGLEEDVLTHIGNVASSVPVENFTI HGGLSRILKTRKELVTNRTVDWALAEYMA 660 SMTCPSTGLEEDILTHIGNVASSVPVENFTIHGGLSRILKTRRELVTNRTVDWALAEYMA 660

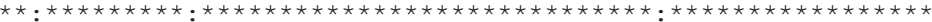

FGSLLKEGIHVRLSGQDVERGTFSHRHHVLHDQNVDKRTCI PMNHLWPNQAPYTVCNSSL 720 FGSLLKEGIHVRLSGQDVERGTFSHRHHVLHDQNVDKRTCI PMNHLWPNQAPYTVCNSSL 720

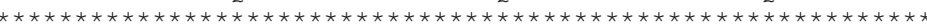

SEYGVLGFELGFAMASPNALVLWEAQFGDFNNMAQCI IDQF ICPGQAKWVRQNGIVLLLP 780 SEYGVLGFELGFAMAS PNALVLWEAQFGDFNNMAQCI IDQF ICPGQAKWVRQNGIVLLLP 780

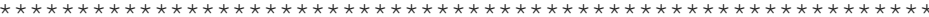

HGMEGMGP EHSSARPERFLQMCNDDSDVLPDLMESNFDINQLYDCNWVVVNCSTPGNFFH 840 HGMEGMGPEHSSARPERFLOMCNDDPDVLPNLOEENFDISOLYDCNWIVVNCSTPGNFFH 840

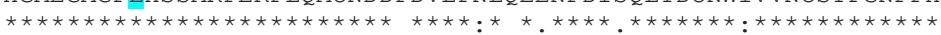

VLRRQILLPFRKPLIVFTPKSLLRHPEARTNFDEMLPGTHFQRVIPEDGPAAQAPDKVKR 900 VLRRQILLPFRKPLIVFTPKSLLRHPEARTSFDEMLPGTHFQRVI PEDGPAAQNPDKVKR 900

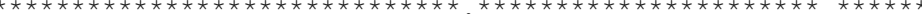

LLFCTGKVYYDLTRERKARDMAEQVAITRIEQLSPFPFDLLLKEVQKYPNAELAWCQEEH 960 LLFCTGKV YDLTRERKARDMAEEVAITRIEOLSPFPFDLLLKEAOKYPNAELAWCOEEH 960

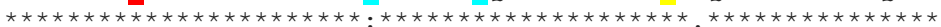

KNQGY PYVKPRLRTTIDRAKPVWYAGRDPAAAPATGNKKTHLTELQRFLDTAFDLDAFK 1020 KNQGY DYVKPRLRTTIDRAKPVWYAGRDPAAAPATGNKKTHLTELQRFLDTAFDLDAFK 1020

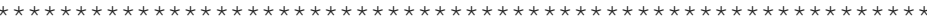

KFS 1023

KFS $\quad 1023$ 


\section{Supplementary Fig. B.2. (See caption for description on page 197)}

ictidomystridecemlineatus

rattusnorvegicus

ictidomystridecemlineatus

rattusnorvegicus

ictidomystridecemlineatus

rattusnorvegicus

ictidomystridecemlineatus rattusnorvegicus

ictidomystridecemlineatus rattusnorvegicus

ictidomystridecemlineatus rattusnorvegicus

ictidomystridecemlineatus rattusnorvegicus

ictidomystridecemlineatus rattusnorvegicus

\# Sequence

\# Sequence

\# Sequence

\# Sequence

\# Sequence

\# Sequence

\# Sequence

\# Sequence

\# Sequence

\# Sequence

\# Sequence

\# Sequence

\# Sequence

\# Sequence

\# Sequence
------------------MLSRSRCVSRAF----SRS LAAFQKGNCPLGRRSLPGVSL MILARLTTRHPTRLVVHSEHPSLLGWCCGRPHFRLLPGP I SGVRPPS I PLAPRCCPGPAA

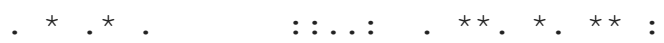

CQGPSYPD-SRKIVINNSSVFSVRFFRTTAVCKDDVITVNTPAFAESVTEGDVRWEKAVG CPGRSAARFLPSRS INNSSVFSVRFFQTTAVCKNDVITVQTPAFAE SVTEGDVRWEKAVG

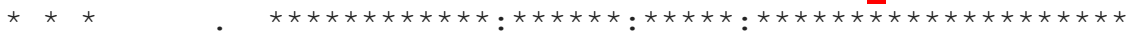

DTVAEDEVVCEIETDKTSVQVPSPANGVIEALLVPDGGKVEGGTPLFTLRKTGAAPAKAK DAVAEDEVVCEIETDKTSVQVPSPANG I IEALLVPDGGKVEGGTPLFTLRKTGAAPAKAK

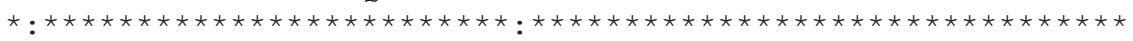

PAEAPATAPKAEPAISAPPPPPAAPI PTQMPPVPS PSQP PSSKPVSAIKPTAAPPLAEPG PAEAPATAHKAAPEAPAAP P PVAPVPTQMPPVPSPSQPPSSKPVSAIKPTAAPPLAEAG

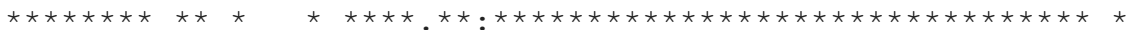

SGKGVRSEHREKMNRMRQRIAQRLKEAQNTCAMLTTFNE I DMSNIQDMRARHKDAFLKKH AAKGLRSEHREKMNRMRQRIAQRLKEAQNTCAMLTTFNEVDMSNIQEMRARHKDAFLKKH

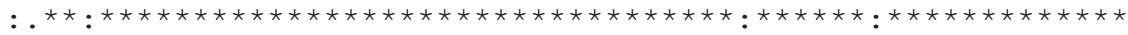

NLKLGFMSAFVKASAFALQEQPVVNAVIDDATKEVVYRDYIDISVAVATPRGLVVPVIRN NLKLGFMSAFVKASAFALQEQPVVNAVIDDATKEVVYRDYIDISVAVATPRGLVVPVIRN

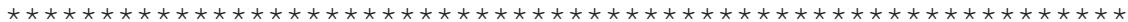

VETMNYADIERTISELGEKARKNELAIEDMDGGTFT ISNGGVFGSLFGTPI INPPQSAIL VETMNYADIERT INELGEKARKNELAIEDMDGGTFT I SNGGVFGS LFGTPI INPPQSAIL

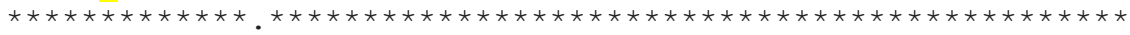

GMHAIFDRPVAVGGKVEVRPMMFVALTYDHRLIDGREAVTFLRKIKAAVEDPRVLLLDL GMHGIFDRPVAVGGKVEVRPMMYVALTYDHRLIDGREAVTFLRKIKAAVEDPRVLLLDL

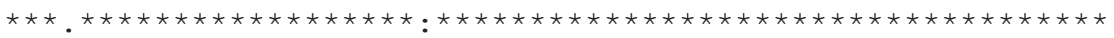




\section{Supplementary Fig. B.3. (See caption for description on page 197)}

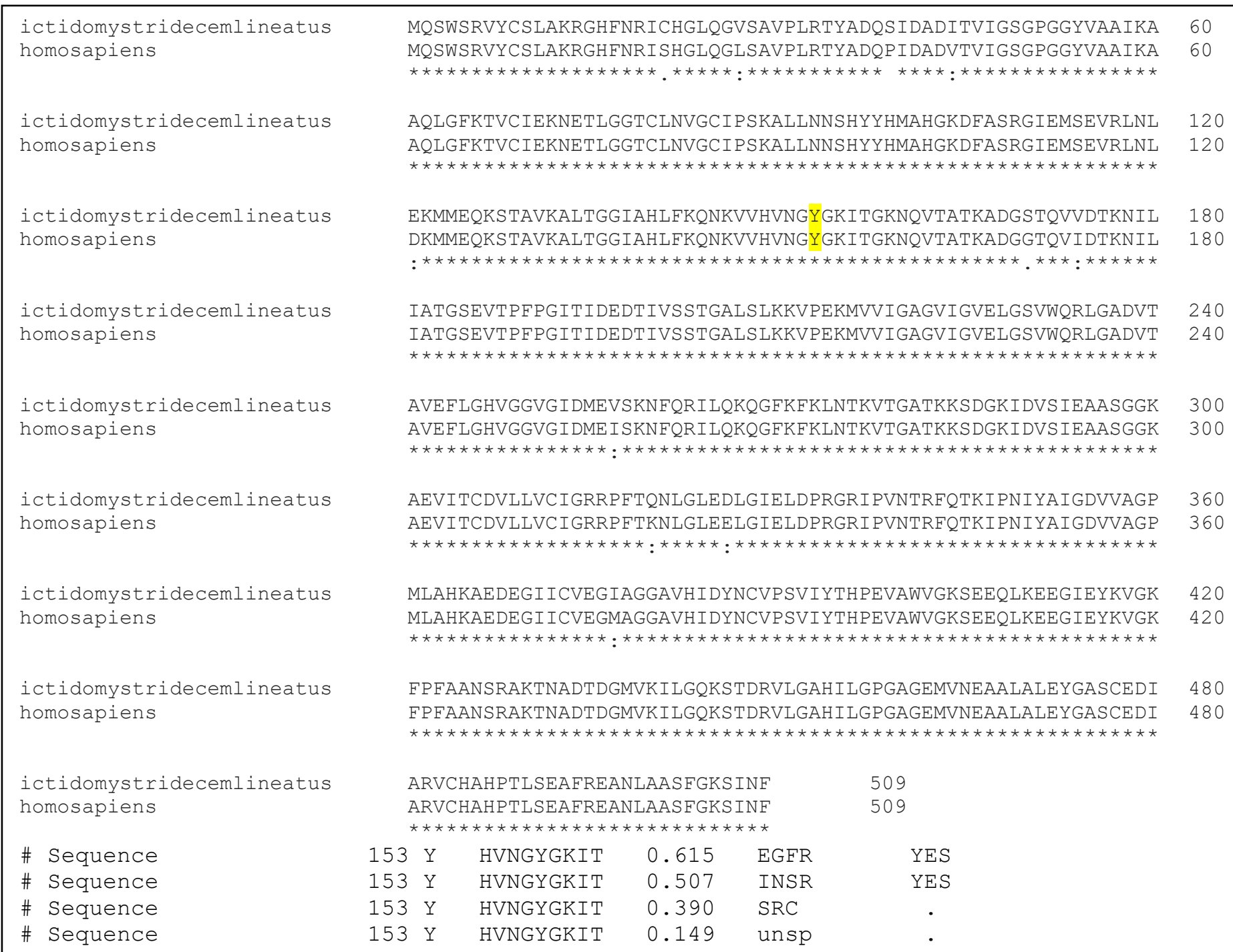




\section{Appendix C: Chapter 5 Supplementary Data and}

Figures 
Appendix C.1. Identification of GDH band in crude lysates compared to purified GDH

Full western blot image (see Fig 5.5) of skeletal muscle lysates from euthermic and hibernating ground squirrels probed with anti-GDH antibody (Left image) compared to a Coomassie stained gel of the purified GDH (Right image, Fig. 5.1). The western blot of the crude samples clearly demonstrates a prominent band where GDH would be expected that is in accordance with the bovine GDH molecular weight standard and is at the same place in the membrane in the Coomassie stained gel.

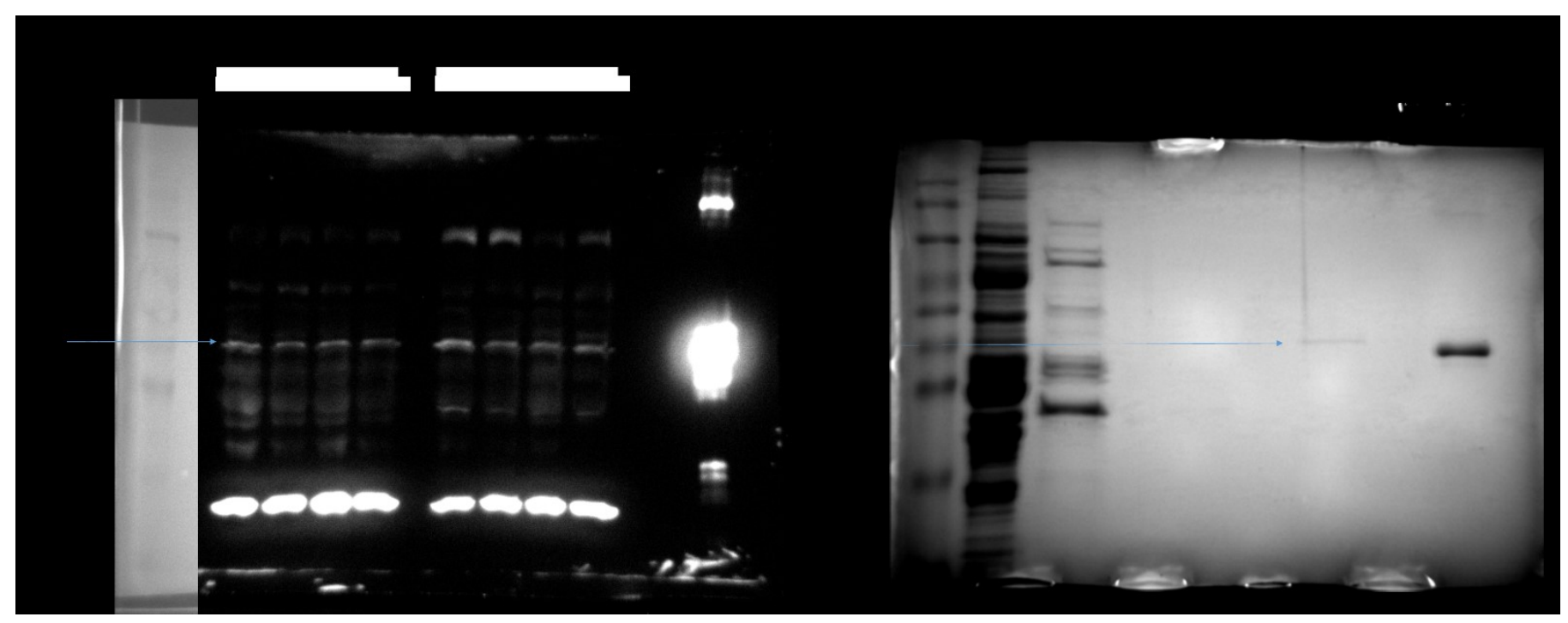


Appendix C.2. ADPredict ADP-ribosylation prediction tool full scoring output for all acidic residues in I. tridecemlineatus GDH sequence (NCBI Reference Sequence: XP_021587637.1). Each residue is scored in three different weights and the average is given as the main output (ADPredict). The three algorithm methods giving each individual score are: Recursive Partitioning (RP), Random Forest (RF) and Support Vector Machines (SVM). Average scores higher than 0.4 were considered putative ADP-ribosylation sites

\begin{tabular}{rlrrrr} 
& & \multicolumn{2}{l}{ AAD } & \multicolumn{1}{l}{ AAD } & \multicolumn{1}{l}{ AAD } \\
Position & Sequence & ADPredict & \multicolumn{1}{l}{ RP } & \multicolumn{1}{l}{ RVM } \\
9 & CAVVDVPFG & 0.24 & 0.34 & 0.19 & 0.2 \\
28 & KNYTDNELE & 0.17 & 0.23 & 0.1 & 0.19 \\
30 & YTDNELEKI & 0.31 & 0.6 & 0.16 & 0.17 \\
32 & DNELEKITR & 0.39 & 0.69 & 0.31 & 0.16 \\
41 & RFTMELAKK & 0.32 & 0.69 & 0.09 & 0.17 \\
53 & GPGIDVPAP & 0.34 & 0.6 & 0.25 & 0.17 \\
58 & VPAPDMSTG & 0.37 & 0.6 & 0.23 & 0.27 \\
63 & MSTGEREMS & 0.3 & 0.61 & 0.22 & 0.08 \\
65 & TGEREMSWI & 0.14 & 0.26 & 0.07 & 0.08 \\
71 & SWIADTYAS & 0.28 & 0.6 & 0.13 & 0.11 \\
81 & IGHYDINAH & 0.18 & 0.26 & 0.11 & 0.18 \\
114 & FHGIENFIN & 0.21 & 0.37 & 0.15 & 0.1 \\
119 & NFINEASYM & 0.26 & 0.37 & 0.11 & 0.31 \\
134 & PGFGDKTFV & 0.14 & 0.23 & 0.12 & 0.06 \\
165 & VGVGESDGS & 0.54 & 0.78 & 0.45 & 0.39 \\
167 & VGESDGSIW & 0.11 & 0.23 & 0.09 & 0.02 \\
174 & IWNPDGIDP & 0.28 & 0.6 & 0.11 & 0.13 \\
177 & PDGIDPKEL & 0.17 & 0.26 & 0.12 & 0.14 \\
180 & IDPKELEDF & 0.28 & 0.6 & 0.11 & 0.13 \\
182 & PKELEDFKL & 0.21 & 0.34 & 0.15 & 0.16 \\
183 & KELEDFKLQ & 0.09 & 0.17 & 0.01 & 0.1 \\
201 & AKVYEGSIL & 0.34 & 0.69 & 0.2 & 0.14 \\
206 & GSILEADCD & 0.49 & 0.78 & 0.39 & 0.31 \\
208 & ILEADCDIL & 0.22 & 0.34 & 0.23 & 0.11 \\
210 & EADCDILIP & 0.21 & 0.35 & 0.16 & 0.1 \\
218 & PAASEKQLT & 0.54 & 0.78 & 0.45 & 0.39 \\
236 & KIIAEGANG & 0.52 & 0.78 & 0.48 & 0.32 \\
245 & PTTPEADKI & 0.57 & 0.78 & 0.49 & 0.43 \\
247 & TPEADKIFL & 0.2 & 0.35 & 0.13 & 0.13 \\
252 & KIFLERNIM & 0.13 & 0.23 & 0.06 & 0.1 \\
260 & MVIPDLYLN & 0.22 & 0.34 & 0.08 & 0.24 \\
274 & VSYFEWLKN & 0.13 & 0.17 & 0.07 & 0.15 \\
292 & TFKYERDSN & 0.14 & 0.17 & 0.1 & 0.16 \\
294 & KYERDSNYH & 0.08 & 0.17 & 0.03 & 0.03
\end{tabular}




$\begin{array}{lllllr}305 & \text { MSVQESLER } & 0.41 & 0.69 & 0.28 & 0.27 \\ 308 & \text { QESLERKFG } & 0.14 & 0.26 & 0.07 & 0.1 \\ 325 & \text { VPTAEFQDR } & 0.39 & 0.69 & 0.25 & 0.22 \\ 328 & \text { AEFQDRISG } & 0.14 & 0.26 & 0.09 & 0.07 \\ 335 & \text { SGASEKDIV } & 0.46 & 0.78 & 0.22 & 0.39 \\ 337 & \text { ASEKDIVHS } & 0.22 & 0.26 & 0.11 & 0.28 \\ 348 & \text { AYTMERSAR } & 0.33 & 0.69 & 0.17 & 0.12 \\ 366 & \text { NLGLDLRTA } & 0.21 & 0.26 & 0.13 & 0.24 \\ 377 & \text { VNAIEKVFK } & 0.34 & 0.69 & 0.25 & 0.09 \\ 385 & \text { KVYNEAGVT } & 0.33 & 0.78 & 0.08 & 0.13\end{array}$


Appendix D: Publication List 


\section{Published:}

Green, S.R., Storey, K.B. 2021. Functional and post-translational characterization of pyruvate dehydrogenase demonstrates repression of activity in the liver but not skeletal muscle of the Richardson's ground squirrel (Urocitellus richardsonii) during hibernation. Journal of Thermal Biology. 99. doi: 10.1016/j.jtherbio.2021.102996

Abboud, J., Green, S.R., Smolinski, M.B., Storey, K.B. 2020. Regulation of an important glycolytic enzyme, pyruvate kinase, through phosphorylation in the larvae of a species of freezetolerant insect, Eurosta solidaginis. Insect Molecular Biology. 30 (2): 176-187 doi: $10.1111 / \mathrm{imb} .12687$

Smolinski, M.B., Varma, A., Green, S.R., Storey, K.B. 2020. Purification and regulation of pyruvate kinase from the foot muscle of the anoxia and freeze tolerant marine snail, Littorina littorea. Protein J. 39 : 531-541 doi: 10.1007/s10930-020-09934-9

Green, S.R., Al-Attar, R., McKechnie, A.E., Naidoo, S., Storey, K.B. 2020. Role of Akt signaling pathway regulation in the speckled mousebird (Colius striatus) during torpor displays tissue specific responses. Cellular Signalling. doi: 10.1016/j.cellsig.2020.109763

Smolinski, M.B., Green, S.R., Storey, K.B. 2020. Characterizing the regulation of pyruvate kinase in response to hibernation in ground squirrel liver (Urocitellus richardsonii). Comparative Biochemistry and Physiology, Part B. 248-249. doi: 10.1016/j.cbpb.2020.110466

Green, S.R., Storey, K.B. 2020. Regulation of the $\alpha$-ketoglutarate dehydrogenase complex during hibernation in a small mammal, the Richardson's ground squirrel (Urocitellus richardsonii). BBA - Proteins and Proteomics. 1868 (9). doi: 10.1016/j.bbapap.2020.140448

Smolinski, M.B., Green, S.R., Storey, K.B. 2019. Glucose-6-phosphate dehydrogenase is posttranslationally regulated in the larvae of the freeze-tolerant gall fly, Eurosta solidaginis, in response to freezing. Insect Biochemistry and Physiology. 102 (4). doi: 10.1002/arch.21618

Green, S.R., Storey, K.B. 2019. Purification of carbamoyl phosphate synthetase 1 (CPS1) from wood frog (Rana sylvatica) liver and its regulation in response to ice-nucleation and subsequent whole-body freezing. Molecular and Cellular Biochemistry. 455: 29-39. doi: 10.1007/s11010018-3468-8.

Hadj-Moussa, H., Green S.R., Storey, K.B. 2018. The living dead: mitochondria and metabolic arrest. IUBMB Life. 70: 1260-1266. doi: 10.1002/iub.1910

Green, S.R., Storey, K.B. 2017. Regulation of glutamate dehydrogenase (GDH) in response to whole body freezing in wood frog liver linked to differential acetylation and ADP-ribosylation. Archives of Biochemistry and Biophysics. 636: 90-99. doi: 10.1016/j.abb.2017.10.010 
Luu, B.E., Green, S.R., Childers, C.L., Holahan, M.R., Storey, K.B. 2017. The roles of hippocampal microRNAs in response to acute postnatal exposure to di(2-ethylhexyl) phthalate in female and male rats. NeuroToxicology. 59: 98-104. doi: 10.1016/j.neuro.2017.02.004

Green, S.R., Storey, K.B. 2016. Regulation of crayfish, Orconectes virilis, tail muscle lactate dehydrogenase (LDH) in response to anoxic conditions is associated with alterations in phosphorylation patterns. Comparative Biochemistry and Physiology B: Biochemistry and Molecular Biology. 202: 67-74. doi: 10.1016/j.cbpb.2016.08.004.

Childers, C.L., Green, S.R., Dawson, N.J. and Storey, K.B. 2016. Native denaturation differential scanning fluorimetry: Determining the effect of urea using a quantitative real-time thermocycler. Analytical Biochemistry. 508: 114-117. doi.org/10.1016/j.ab.2016.05.019.

\section{Submitted/Under Review:}

Green, S.R. and Storey, K.B. (2021) Citrate synthase in hibernating Richardson's ground squirrels (Urocitellus richardsonii) displays altered functional properties. Cryobiology. Submitted April 7 2021. Manuscript No. CRYO-D-21-00094

Green, S.R., Al-Attar, R., McKechnie, A.E., Naidoo, S. and Storey, K.B. (2021) Phosphorylation status of pyruvate dehydrogenase in the mousebird Colius striatus undergoing torpor. Journal of Experimental Zoology Part A: Ecological and Integrative Physiology. Submitted May 19 2021. Manuscript No. JEZ-A-2021-05-0091. 